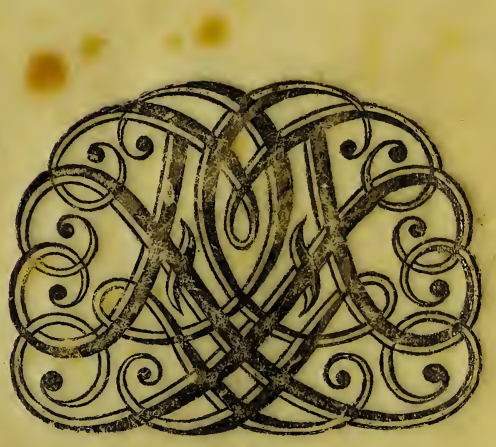


Dez laturicuscikenden - Gefellfiriaft des listerlandes au Alterifruagy verehat d, the Jillilsss Aclolphotzatfisch.

452055 ex $y$ x

4. Loolition arm

$7 m 12 / 35$ 
Molat histo 30

11) 2225

2.) $23 \mu .2045$, Kit I Taf. 



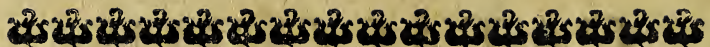

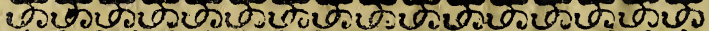

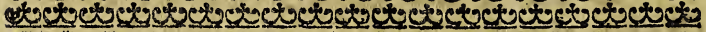

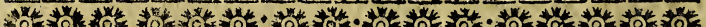
ro

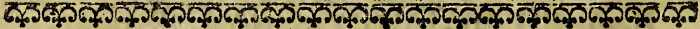

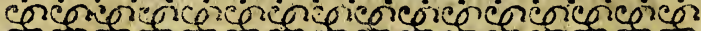

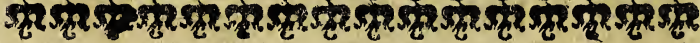

\section{Sietluttefter sisefer.}

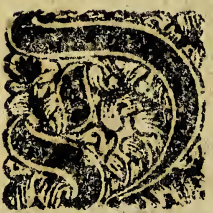

8

Ie mibfame uno tofte babre Collection Deg Spe: nexifden Naturalien $=\mathrm{Ca}-$ binetsitf fo woblgeratben, Dafe es Dem feelig. Manne nid)t wenig SRulbm bey allen Curieufen Riebbabern folder Seltenbeiten zu= twege gebradot bat. (E⿺ find aber ber= fobievene Urfadjen, weldje die gefalnte Exben deffelben anratbon, Dafi fe ferbi= gró entweobergants an einen, oder aber per modum Auctionis ein iedes Stút Defielber an den meiftbietenden zu ùber: hafien emtaloffen find. Solte alfo Se: mano SGilieben tragen, diefes fo wobl choifurt uno müklitge Cabinet ganks toie felviges in undyftelsenderm Catalogo irm. frindidi) recenfirt witb, iberhaupt 


\section{(0)}

fáufflid) an fưbu bringen: So erfutfet man Denfelben bienftliaff fid lángftenb 14. Tage vor inftebender Michaëlis Deffe diefer Jabres, nebft 23ermeloung des Pretii fo er Daran wenden wolte, entweder forriftl. oder Durd einen $B_{\text {es }}$ vollmádytigten bey Sorn. Plsillpp SReins bard Spenern in soerlin zu melden: weil in Errmangelung defien man fontit entid) lofen ift, diefes fo múb/am zufams

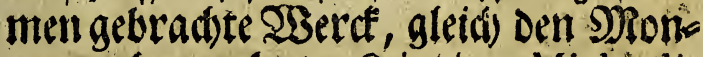
tag nad) ertwellynter Reiphiger Michà̀ëlis Seffe unverzúglid ourd cine Auction 3u diftrahiren.

Taddffethender Catalogus twiro gwa eines jeden aud Des Fleiniten Studtgens befielben (Frwebnung tbun; Obgleid) Der Geneigte Sefer an Der gemadten. Sronung viel aus zulesen finden modi. te: Allein der Sod diefes berúbmten Mannes iff groffen Theils mit Sduls Daran, weil er gleid nad) Translocation diefes fo zahlreiden Cabinets bon Demfertben úbereilet wurde, und es alfo nidet felbft rangiren fonnen. Dif (E) ben Deffelben bácten zwar Dabin be

Dadit 


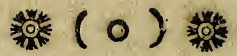

bad)t fenu fotlen; weil fie co aber zu bes

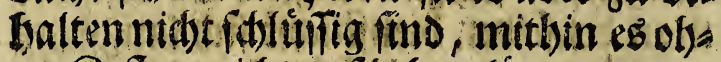
ne Roften nid)t gefdeteten fonnen, am allerfdyerften aber des funfftigen $\mathfrak{B}$ e: fikers Sinn zu erratben war, fo ba ben fie diefe tleine Snutbe lieber dem. zufúnffigen Som. Beftzern úberlaften wollen.

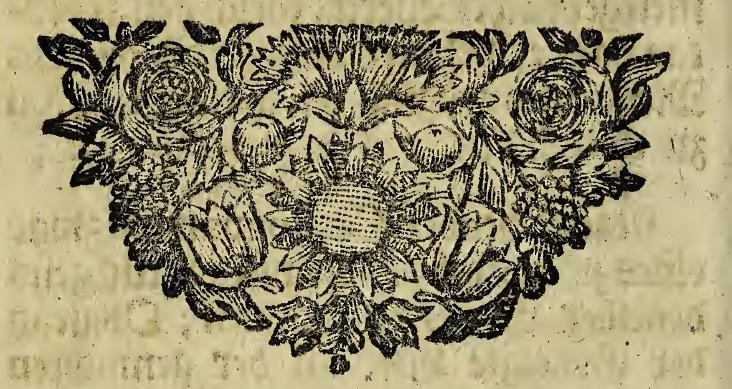




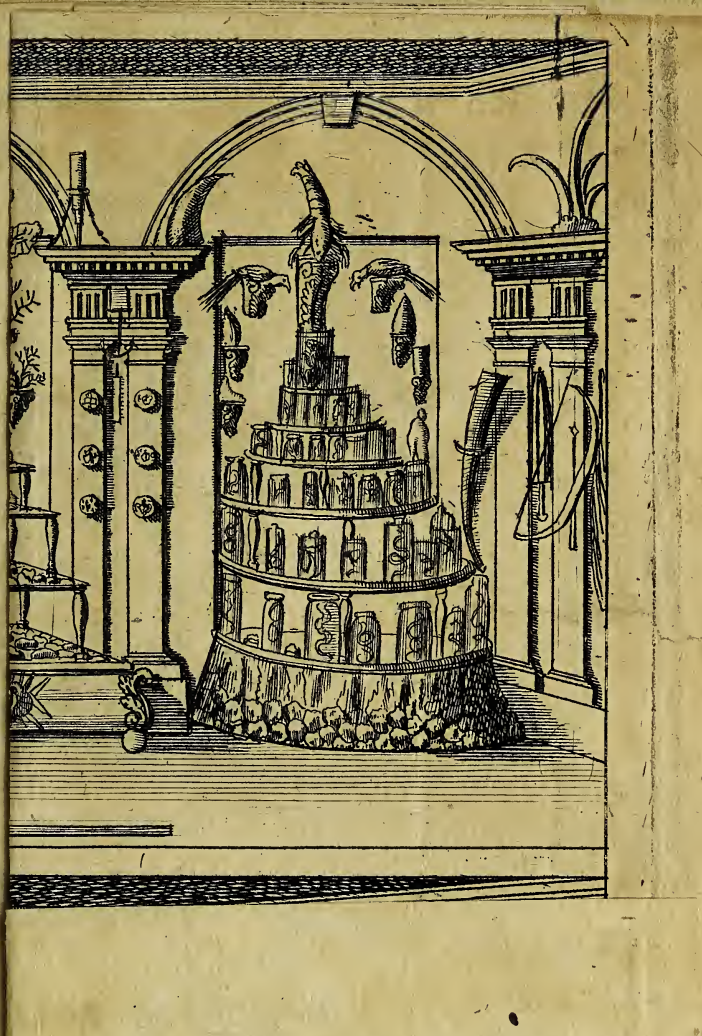





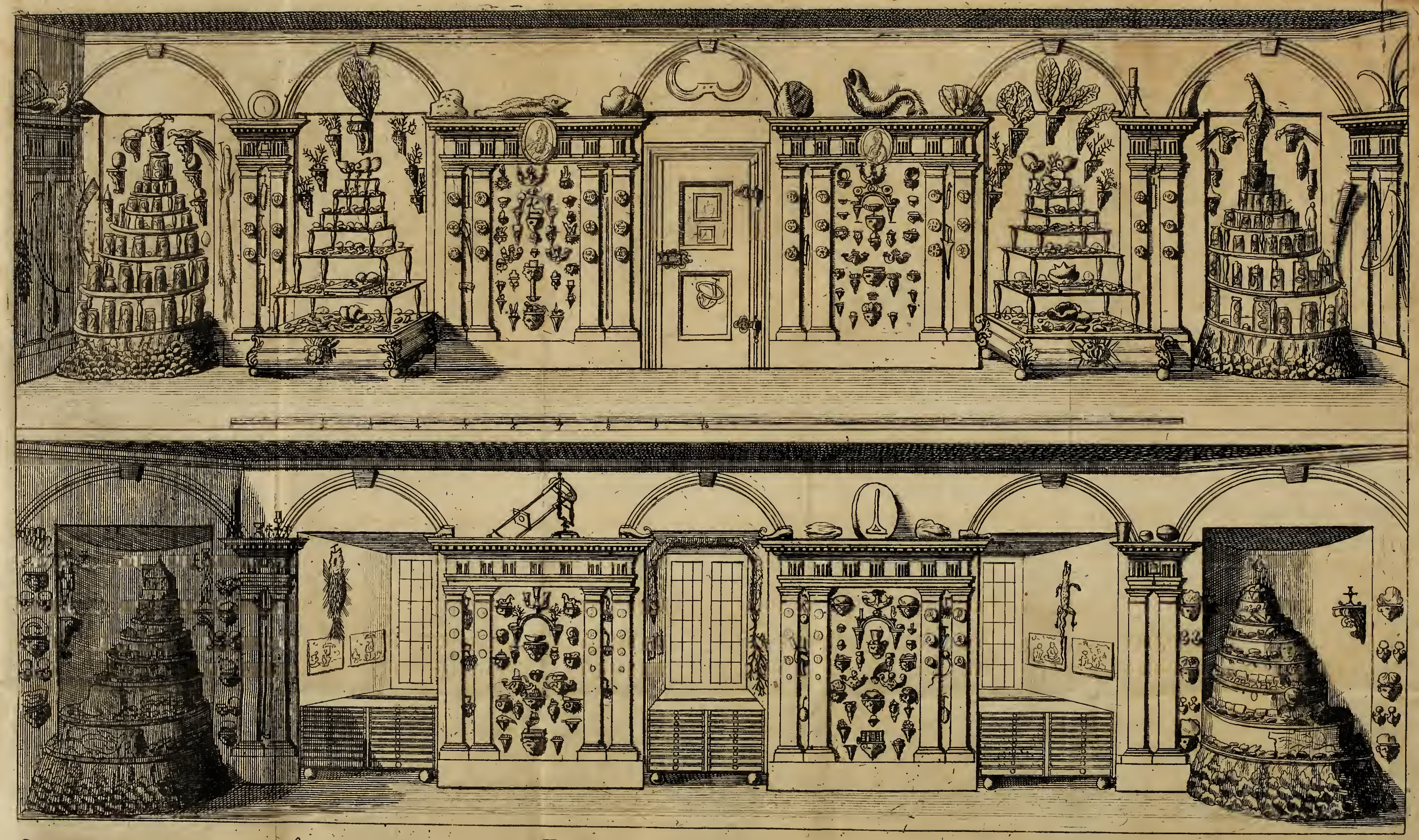

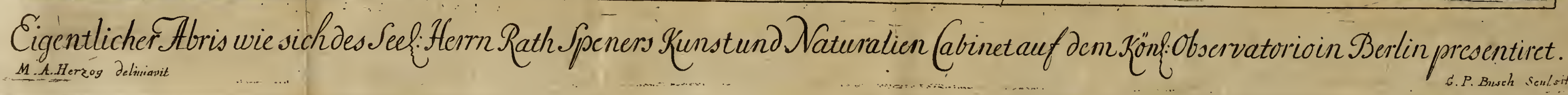




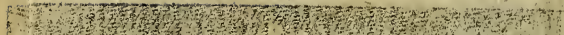

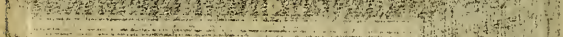

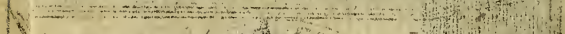

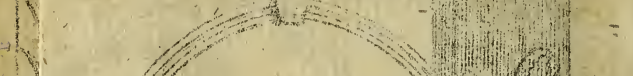

1 if

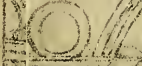

tint

$\rightarrow=-14$

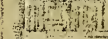

$x+\frac{1}{4}+\frac{1}{2}$

(1) 1 i 1 -

औ

(1) 10

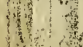

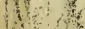

(1)

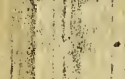

in

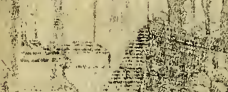

负.

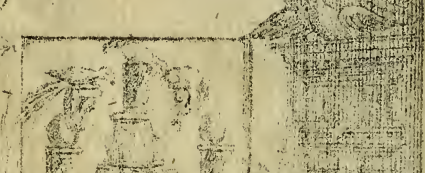

-

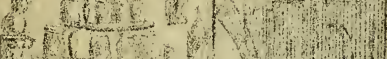

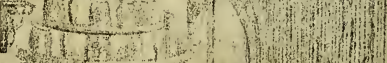

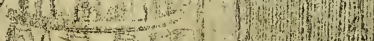
(E) की

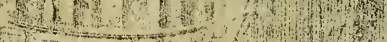

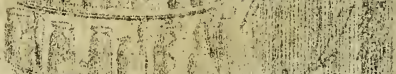
Po,

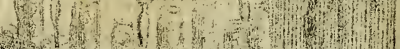

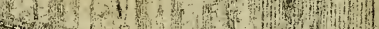
$4+14$ 


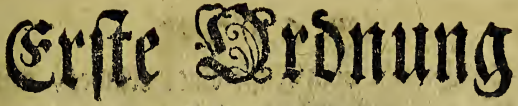

\section{bailt in fich}

\section{Stlletband rare Dinge in eis}

nem $B a f a m i d e n$ Spiritu

\section{coniervirt// \\ Zon}

Sqenfiben/ ₹bieren/3igelm!

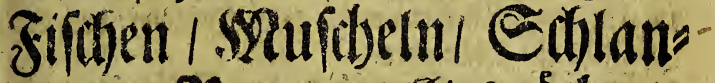
gen/ Taupen / Gerwid) : feII/ U. o. gl.

\section{I.}

(920 In Abortus mufculus yon 6. Matinth cum Q) E placenta uterina, Deffen \$ruft geóffict / Damis (2) 2 man Den Drth und die Sivófe Des Setrecens beffer etfectunen móge.

2. शPod) ein Americaner bon bergleidjen Giofife.

3 Sin anderer bon vier Syonatl).

4 Nodf) ein anderes bun bren s)ionath.

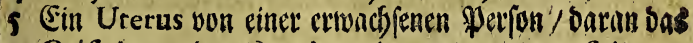
Orificium, die Ovaris uno extremitates foliaces wobl zu felien.

6 Eine Shand von einem Sinde/ fo am bikgigen Fieber geftorben / baran Die Epidermis abgefondert. Shaile.

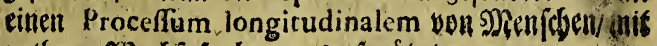

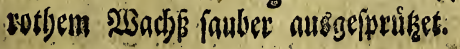




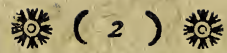

7 Die Epidermis yom GutTe eintes sinbes / mit Denen PRågeln zart nbgefoundert.

8 Eime Grintándifbe Fledermaus cinen Fus lang und rótblicl) am Fsarbe.

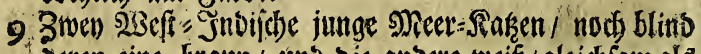

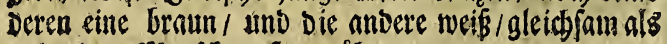

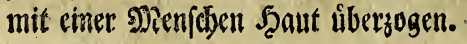

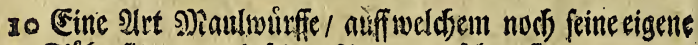
Fllofe fižzen/ uns peine 2lugen ju jeljen find.

II Sin Dft = Inbififer Crocodil, von Biejdlectete ein siseibdien / mit fleinern Ropffe / und Dundtern Farben als sie Africanifoten.

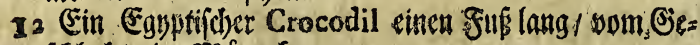
(jd)led)e ein Miánndjen.

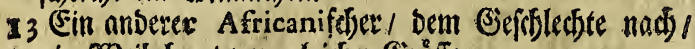
ein STsetberen / won gleidher Givolfe.

14. Ein fleiner Inbianifher (Tro = Crocodil, wie ein Chameleon voer Siothegber geffaltet/brânlid) von fSarbel mit einem langen Edjwanţe

15 Ein Afiatifid Chamæleon mit einem langen Estwane

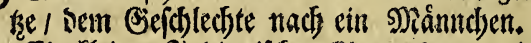

26 Ein fleitier Indianifoer Chamaleon mit andichten Siliden.

a7 (Ein Chamaleon mittlerer Brioffe nit einem jacticteten Siúflen uno fehr langen Eđtwwankze.

18 (sin dito grines mit fulbid)en sopffe jactidjten Sirtiten umo rangen Esfrwanţe.

79 Ein mittelmáfígiges Chamæleon vin Der 2teth / po bie Santben veriniern $/$ welthes nodf alle Coleuren pielet. 20 Ein gants gruines / dorfo non ferrato.

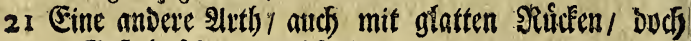
an Gieftalt fehr unterfosicben.

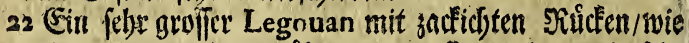
ein Chamaleon, hiber sie maffen ralbs, uns febr curicux.

2.3 Eine Legouanen 


\section{柴 (3)}

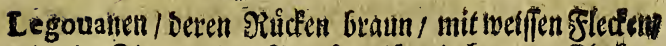
wie ein Sieger / ber Saud) meis mit braunen filecten.

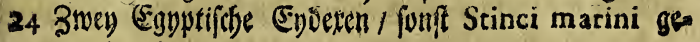
nannt.

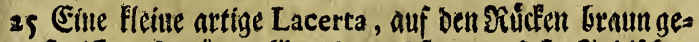

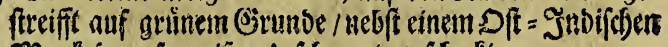

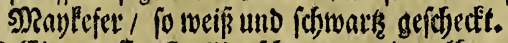

26 Ein gruffer Stellio fdjwats mit gelfen fflecten/ bäle

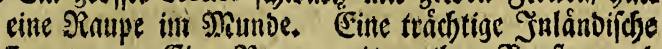
Lacerta. Eine Raupe mit rotben Surtfen uns Sdjmantre. Eine 2/rth/ Des Amerianifden Cerei ober Igel=Eienádbjes.

27 3wen Europzifibe Salamander, einanber umb. ftufend.

as Eine fliegende Jnbianifore Endere/ fouft Draco volans Clufii genannt.

29 Eite anbere etwas groffer/ aus Inbien.

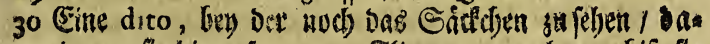

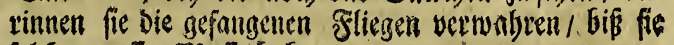
forke zu effen shiffie baten.

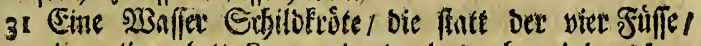
bier artige platte Extremitæ ten bat/ ofne einige Mar-

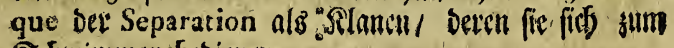
Silinimuen bebienen.

32 Jf nod cime bartu fummen.

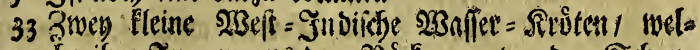

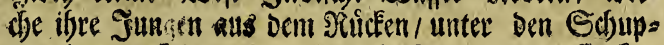
pen bervor Friegen/ wie Dergleisen eine groffe bed

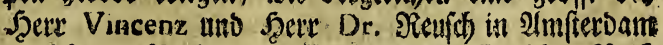

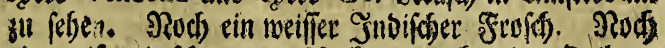
eir weiß und fobmars gejofedte won eben Den Dith.

34 Ein flein $\mathrm{Sec}=$ \$ferdoffer/ fonft Hippo campus Clu . fii Benanut.

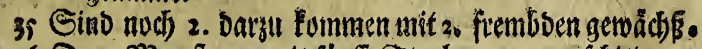
36 Dren sicerferne mit fünff Stralen / von berjobiebenes Artb.

$$
\text { II } 37 \text { Gin }
$$




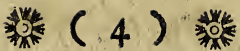

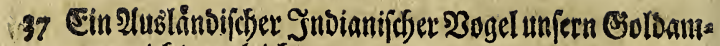
mern nidft unteleidf.

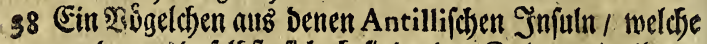
won Denen brielbft fich befinbenden Spinnen in ibremGelvebe gefongen / uns gefeffen werden.

39 Ein Scindsen mit 4 . Fúfifen und 4. Flügelw/ Ao. 1698. in Sierlin autsgelsedt.

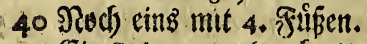

41. Ein Polypus oder bieiter Blade - Fifd) nod mit bem

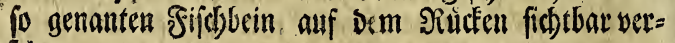
(el)en.

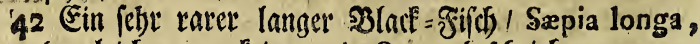
- Dergleidjen bon Feinem Auctore bejdyrieben. 43 Fin Fliegenter Soering.

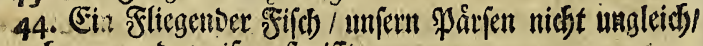

braun und weíp geftreifft.

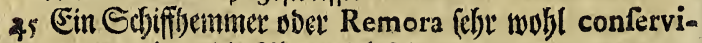
-. ret/ bon bem bie alten viel fabulitet.

46 Sin dito fleinerer.

47 Ein 'vituedfidjter filid)/ jonft Thurus Marinus ges nount bat vier Scorter 2. votne und 2. bintell.

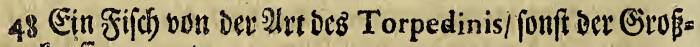
fopff genannt.

49 Einganksplntter fleiner bunter See, Jif filien felir artig.

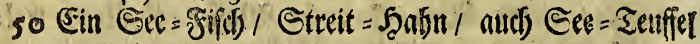
geriannt.

s1 Sict Chinefifdre und Japanifore Bjold = tmo Silbet: Fijblein / von serfojedener Girofie/ babey eine (E) = wádfic / fonft Pulmo Marinis genannt.

52 Srev Eee fijhe vont bejunderer Irt.

53 Ein molfl confervirter Pifcis rhomboidalis, furt Chamelis genannt/ Deffen enfferfter Eljeil brnum/mit lauter beflen Gternen bemerclet.

54 Ein Fiffh/ fonft Acus Maris genannt/ nelff einem felds baren s) seerfteme / Darinnen nod sia fleiner in bee

SNitten 


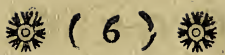

7o Eine lleine Oft = Inbil(d) Ed)lange/ gant bart unt

rundt/ /pringen von ein anber/wenn man Darauf (j)/

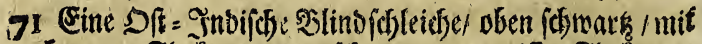

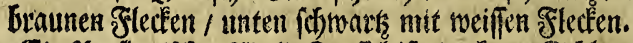

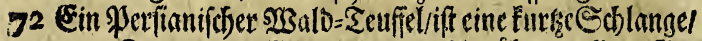

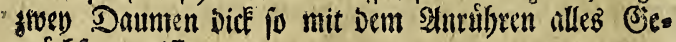
wádje veresiftet.

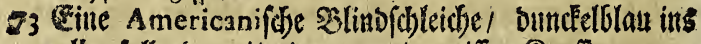
gelbe falleno / mit einent ganks weifien Siopfie.

74 Cine Amexicamifde Seflange/ aus Der IItty Det Vipern, bratan / mit Eleinen weiffen Glecten / unter foswarers und weir.

G5: Eine anbere Heinere/ autd) ans Ginbien/Fellbrautm/ mit

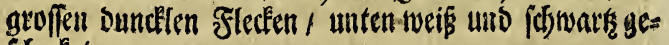
fichectet.

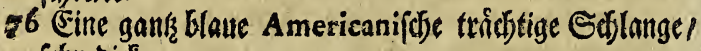
ferbi bick.

77 Eine bafb weib uns braume/ eflifise Ellen lange Ameticanifbe Elange.

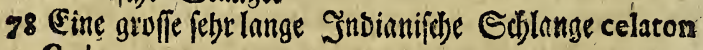
Coleur.

79 Eine groffe fardle raffel soer Elapper Edylange.

80 in:ovel cins.

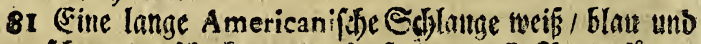

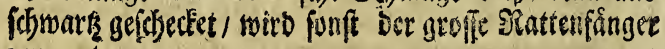
senannt.

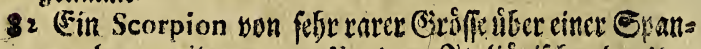

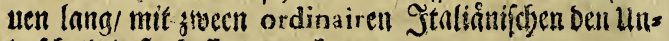

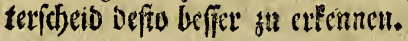

33 (Ein Tunetanifjer Scorpion ands) bon jeentidfer

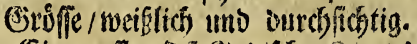

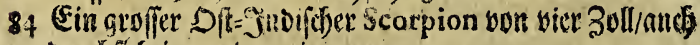
Demrchifich is $/$ mie woriger.

85 Civie faff vier Zoll lange Scolopendra vere Taufcno, F) B.

85 Sine arroffe Epinne aus benen Antillifdsen Infuln mit jellen 


\section{赫 (}

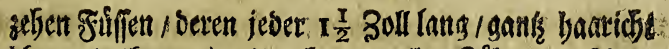

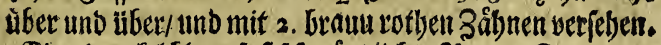

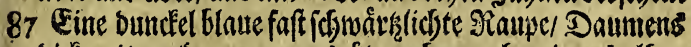
Ditf' mit rotben Extremicấten / bey nabse cinen balben Edsull lang. SEise andere branu und weißs artig ges ichectite.

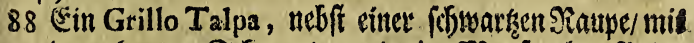

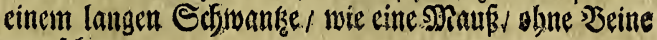
anjuf(b)en.

89 3meene Giolofutbene Dactyli Erucarum, becen einer

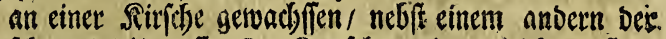

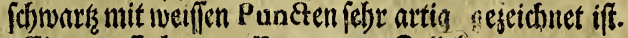
90 Eine groffe braune Eruca an 5, Boll Lang / unten aels bidft uno rauch. Sime andere gelle mit rothen fles

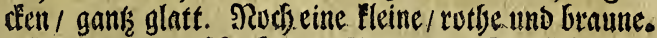
91 Eine Amboinifose bratne Эiaupe mit Stadjetin / wie

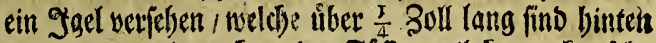

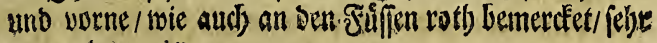
$\mathfrak{r a r} \mathfrak{n n d}$ curiös.

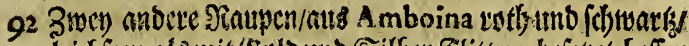

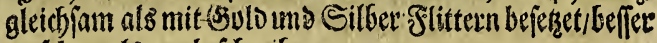

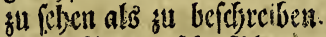

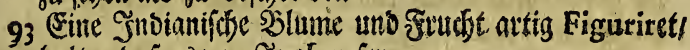

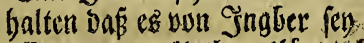

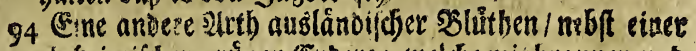

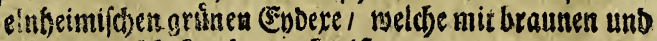
fithwarteng glecten benterdet ift.

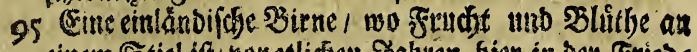

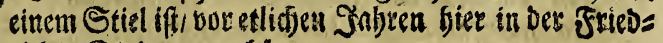
tidess, Stabt grwadjien.

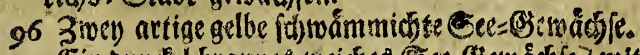

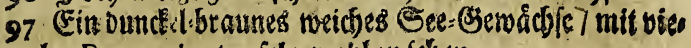
len Exrremitxaten febr woblt gu fêten.

98 Drey Aloë 2 lumen/ beren eine aus . Jolland/ bie anbern benden aus Reipzig.

99 Ein Stellio mit cinem Doppelten Esjwant fersc rat.

$$
\text { शै } 4 \text { los (sin }
$$




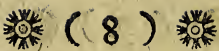

Spo Cin Polipus marinus Deffen Strablen mit 2. शeilfen

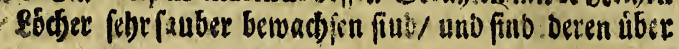
100.

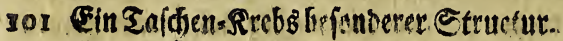

102 Gin aroffer Bopel I fo bim Gouverneur anff eituet Snful in Africa alse eime befondere raritåt gefd) ndft.

I03 4. Stưd Braflianifon blaft Fijogen , baber bert ubern bem sopff alff benseu Eeiten cinen balben פRono / uno am Edjwank ein gleidfals [w warkes Ina doer Spisael.

1045 . Quajanumbi.

Ios 4 Stúc Intianifhe Taufadfüfle / roer Scolo.

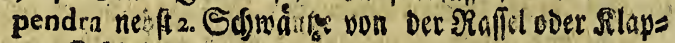
per: Sothlange.

106 Enegrofle beflitbe Africaniffe Edlatige. Ioy Ein wabres uni aufirid)tiges Chamzleon fojón conferviret.

108 5. Sdhlănglidien ober Ed) efis:Dtern mit 4. Fiffen von verjutiedenen foinen farben.

109 9. Stücke artiget See: Fifue/ reben faft wie SBlatts

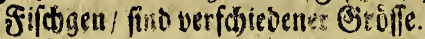

I 3 .

II Ein dito mit noef jwen Hetnern.

212 Cin groffer bell = und oundels blater Legouan febte ichon.

II 3 Eine befonbere 2 rt frembier grauliditer soentexen/

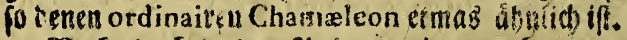

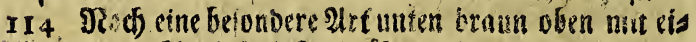
ner etwas blaulichen Jantit fiberggen.

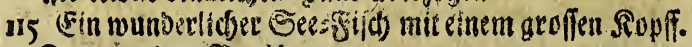

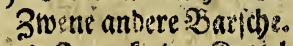

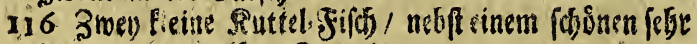
langen gankz gelben Ecurpion.

117 Erre $21 \mathrm{t}$ einas groffa Chamaleon weiflid)! / mit braunen flecten / weif: blaulichten Sopff uno felse langen Sownans. 


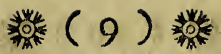

I18 Ein febr Yanger befonberer See-Sitebs von Solläns Dern Siblangen R nig genanotl bat an ftat ber Soderen P. $l$ im = swetuge figuren.

In Ein juig Stadjel: Sw)wein nur eflidier Tage alt. und bas bockidson btel und lange Stadjeln.

120 4. Stuidf frembo artig=gefrerffice und coloritte

Sthlangen.

121 Eine or dinaire $23 a f(e x=$ Solange

122 Gine fojine oben ber blaulid)t / am Sauds aber gaus meife Soblange.

I23 Ein Aborcus van etlits Tagen.

124 Cinfalfiber Abortus inie cine Sraube.

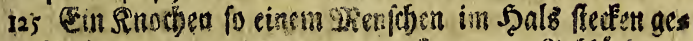

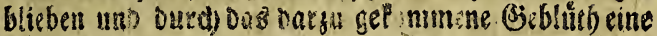

Fifob) (S) falt formiret uno bernads aafigebrocben morber.

126 Ein Canarien=:Sogel obne Fliegel bon Diefem Jaft.

\section{Indere Srontulits \\ bâlt in fich)}

Durd Gaden / Die theils rar bon $24=$ beit / theils als aus = uno cinlán Difde/ alt s und nelle Dinge

\section{Curi}

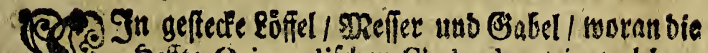
O) - Seeffie Orientalif(cher Chalcedon / in welldem (2) 28 eine rotbe Jafpis=21ber / ein Infe Etum, als eis ae Siene eigentlid) vorbilvet. Die Stiele fino 왕 5

bon 


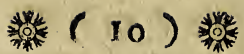

von 2lgat mit vergulben Silber gefaffet. Cin curieux Stúd mofuir mefrmablen 100. Ducaten ges bothen:

2 Ein bon Madame Braunen ans buntem $23 a c t i s$ pouffirtes Portrait in Buffo febr twobl uno funfflich) gemas det. Ift bas SBildonis bez feel. Poffefforis biefes Cabinets.

- Cin ander von Seipel mit Touche gemafltes Portrait gleidefals des Serrn Poffefloris.

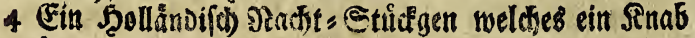
fo Epilepticus za ŞSismar / ber nut 4. Tage mablea gelernet liuffitidi verfertiget.

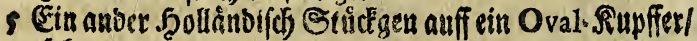
fefre twobl im fFeuer smaliiter.

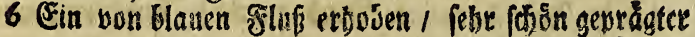
Nummus Jugubralis auff Dic Englífote Rỏnigin Maria.

7 Ein dito vou Guftavo Adolpho in wris Clap fet: fauter.

8 Sine funff 3oll lauge $2 \frac{3}{4}$ Soll breite Scjale aus einem Stúd Agath.

9. Eike Eleinere ald Fafen geftritten.

10 Ein ju einem Dectel einer Sobacts = Dofe fafen weife

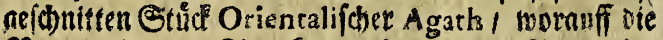
Natur mit einer Zinnuber rothen Jafpis, Noer / eine vollfonmene Davios şarffe felfr curieux vorftellet.

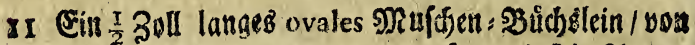
Orientalifofon Calcedon mit gruinen Jafpis-2loetw/

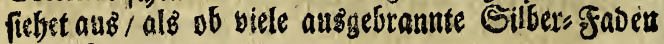
baburci) giengen.

I2 Eine aus einem Stưf Marmor von 2rttfen Dendri-

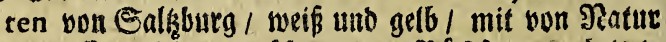

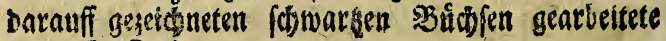
Tobacts: Dofe.

I3 Eit Floxentiniffer Stúd Marmor auft meldjem sine 


\section{粦 (II) 耀}

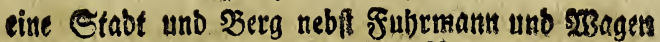
geviltet / wiewoblen bie Siunfi ber Natur in etwas jur Şillfife Funmen.

I4 (a) Esine Buchje aus Therbenthin= Sttin mit Zitts

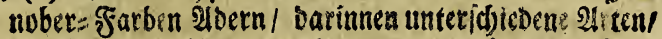
ausländicber/ blenerner / leberner / und jonft meiftens פMofcomitifoter DRünţen.

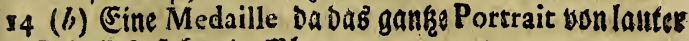
Priapis beftelyt in Sbley.

14 (c) Eine Medaille Da Deos, Bello. Fatigatos,Alma. Pace \& Artis, Cultorem, Regiis. Ditat, Donis aufs

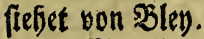

14 (d) Eine Patron von Der Eefangen = Nefmung Shrifti in Bley.

is Eine Siutfore mit 6. Pferben und 3. \$erfonen/ aus Sfelfenbein / fo fauber gearbeitet / vaß fie aucb) cine fliege fortsichen mag.

16 Ein Crucifix mit Johanne und Maria alts \$3udso

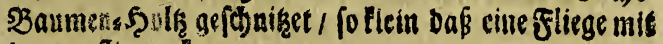
Datbonfliegen lan.

1) श2ad) ein (auber Crucifix aus belfi nbein.

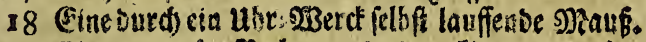

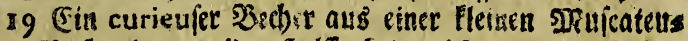

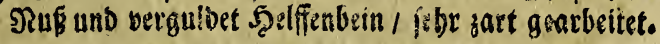

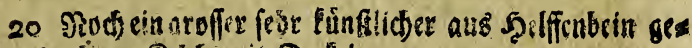
Drebeter Relch) mit Deffil.

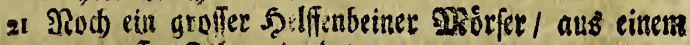
gar grofien 3 obra yeorebes.

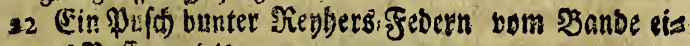
nes balfa cus ungarn.

23 E in Daumen Did" geoseffeter Etrief/ melôten ber fo genannte Engliphe Simjon in meiner OSegenwart is Scollano jerriffen.

24 Eine Inotanifos SRuf fegr wobl politet.

25 Ein Quifo unn gefponteetten \&lafe.

26 Bier glậlerne Sartyri Aquatici. 


\section{耀 ( 12 ) 橉粼}

27 Eläertrne \&uffit Pedartert.

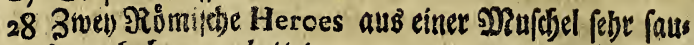
ber erfoben gearbeitê.

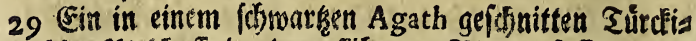
fotes \$itichafif in einem filbernet Sing g.faffet

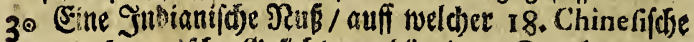

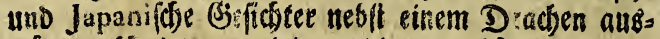

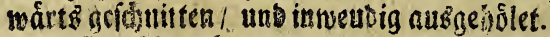

3I [u] Eine bla e fin und wieder mit Gollo gegierte $\mathrm{P}_{\mathrm{a}}=$ nacea Chinenfis। weldse alä ein Amulezum ance. banget wer alle Sirandheiten fenn foil/ riechet fefer

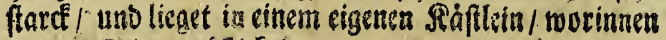
fie aus China gefotididet.

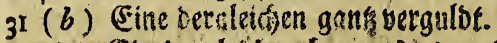

$3^{2}$ (a) (Sin Derglecidsen braitnes Amuletum mit Sins

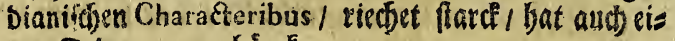
nen Gaven zum nnbáncéen.

32 (b) Ein Dergle diden Dundelel.

33 Eine ancere Maffa Medica rotfee you Farben / und

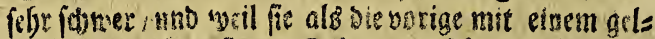

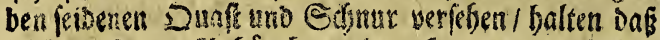

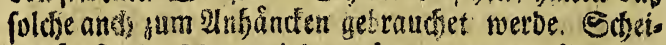
net forft ein Mercuriale $z$ f f $m$ n / und exciditet ein

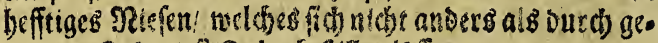
meinen Stinupifi=Zobace fitlen laffet.

34 Eine bergleicten Maffa Medica Darbon föon etrwas verbraudet.

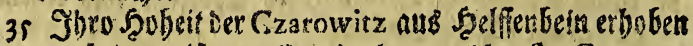
gearbetitet / if eta Original bon Monfr, Govin gll 5Tivfali 1703. verfertigit.

36 Ein Agath jum Couteau de Chafe gemachs / mit \$urpurerotben 2tbern.

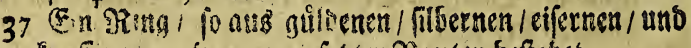

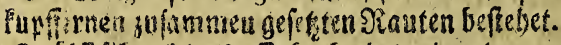

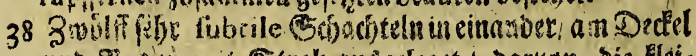

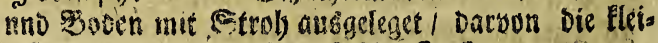
nefte faum ein Sinjen Jisntr befólieffen Ean. 


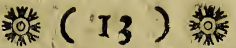

39- Arminius Róniz ber Teutf(den erfyoben auff einer Muf fisel istor fauber gearbeitet.

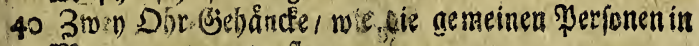
Movicall zu trages kifigen

41 Ein Epaniides Amulet aus einer rothen Eorall ges

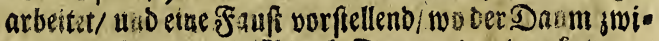

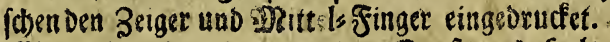

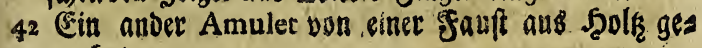
marfest.

43 (Ein Amulet; wo auff eine: Seiten / J N. R. J. uno TETRA GRAMMATON, auff Der andern: IMMANUEL und ANANISABTA DEI gu lefea/ von Silber.

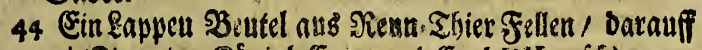
mit Zinn eine Rơnigl Erone und Earl XII gejfilungen geffictet.

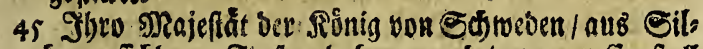

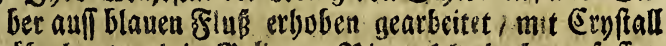

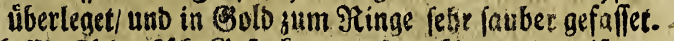

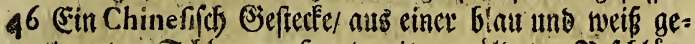

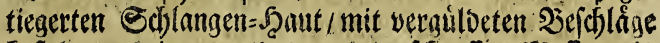

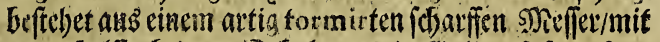

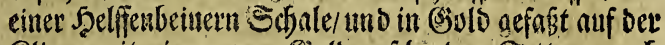

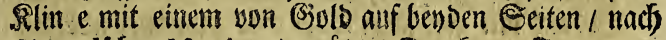

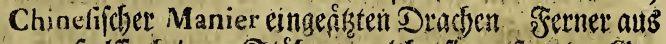

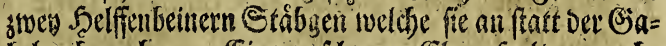

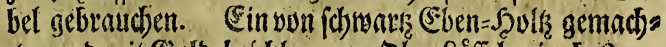

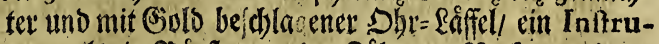

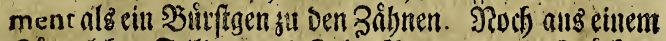

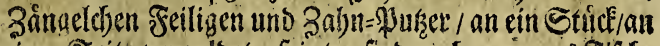

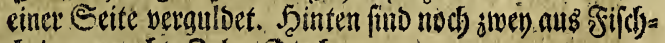
bein gemachte Zabn= Etecher.

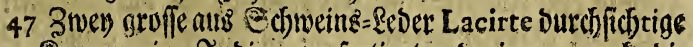
Siepgen in Jnsien verfertiget / Darinnen uods Dic

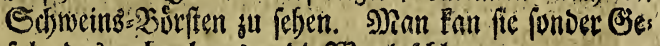

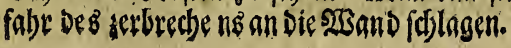




\section{紫( $\left(I_{4}\right)$ ) 繁}

48. Cin in Japan nus einem Echino marino, ober Sce

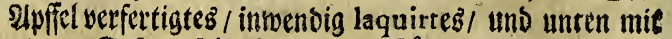

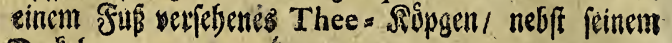
Dencit.

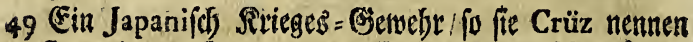

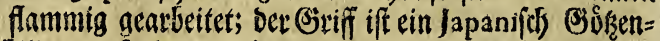

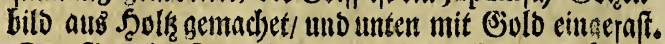

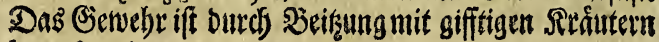
damafconiret.

5o. 3wo aus jartenbinfen geffoctitene laquirte und wergils Dete Japanif(se Esfluffeltyen.

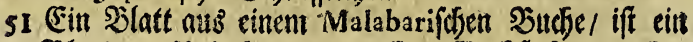
Blatt yon \$almbaum barauff Die Suddffthben mit InAtrumenten einsefrivifelt.

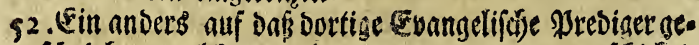

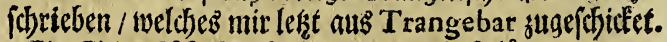

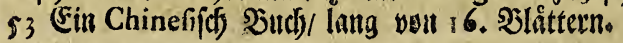

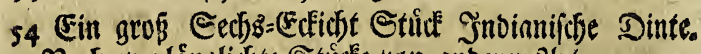

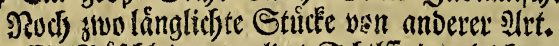

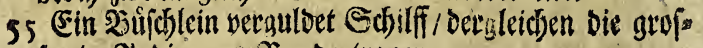
fen in Snoien am Sambe tragen.

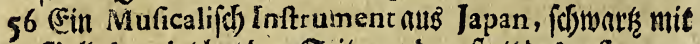
Bulb laquirt bat brey Sciten uhd an ftatt bes refonantz

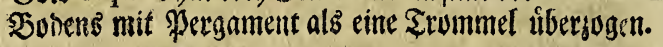

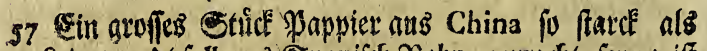

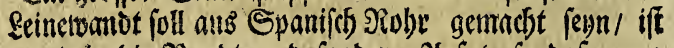

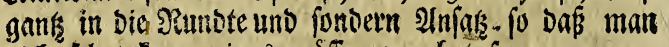
uidost felben fan / wiees múfie gemadfet fevn.

88 Sin Inbianifiber Spiegel von Sprauter gemadjet? auf einer Seite glatt poliret/ und ouf Der antbern mit

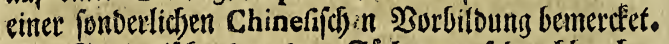

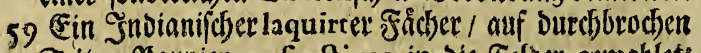
Eeiten Pappier perfpectives in Die Felber gemablet?

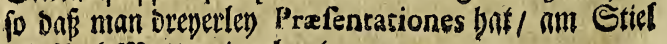

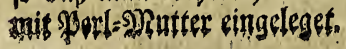




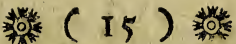

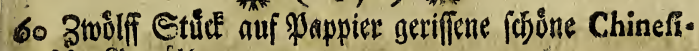
fibe Bemál De.

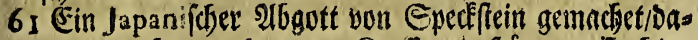

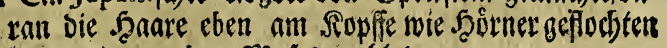
fino / wie mant ben shopes mablet.

62 Ein yon Terra Sigillata gemadifer Tartar, if cin

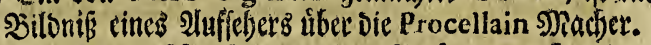
63 Ein Chinefifther Procellain = \$Racter aus eben Der pelben Materie verfertiget.

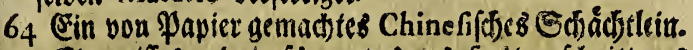

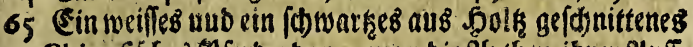
Chinefif (d) zanumung iefien fan.

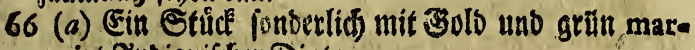
quirt ज̦tobianif(d)er Dinte.

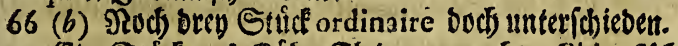

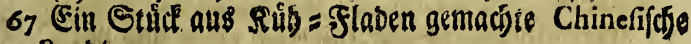
Runite.

68 Eine artige Turna Sulis/ weldbe bie Ebinefen ftatt bee

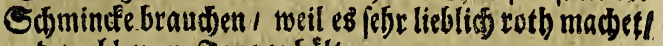
uno mob! gwey sage angalts.

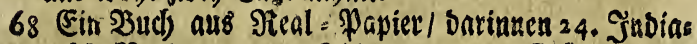
nifote SRationen/ 17. Thiere/ und 52 . Reciuter auf Dem Capo de bonne Efperance nach Dem leben Das feloft geseidinet.

7o Zneene Japanifde Sdube/ beren einer rotb/ ber ans

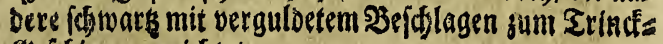
Befojier jugeridjet.

71 Eine Mobriftie Tobacks. Pfeiffe beren Sopff aus 2Reet-Sd)aum/ her Stiel bon Sobr uno uber oren uno cin halb gun lariz ift.

72 Eine Surctifdbel farcter twie bie vorige 4. Fub lang. 73 Eit bon sarter Reinmano mit rotben ₹affent ausige madtes un' mit (E) føes Sembte / mis senen Darju gefobtigen Scofen / wels -

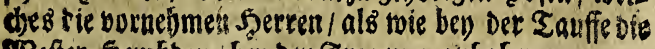
essefter Serabben / bey Der Trauung anjaben. 


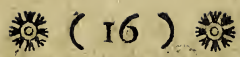

74 Ein Mofcowitifdorer Duaft/ meidjer bey ibnen als

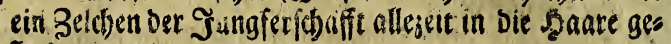
floci)ert ivizo.

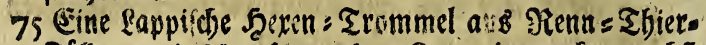

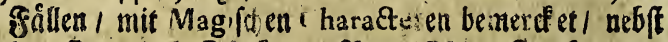
Den Srimmel= Stodé nus Sienn- Sbier. Thodfen mos. van Ş

76 Ein \$aar smofcomitiche Erautenzimmers Pantoffeliil mit Scuff:eEifen befolagen.

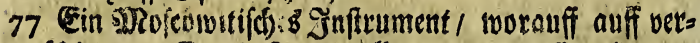
(d)ieveren Dratb)= Sinien gelbe uno rotbe Corallen ges zogen/ fo fich bin und ber idieben la fen i roiro ben ilgnen zum Sledinen gebrandiet.

78 3tveene Soppifd) 3 zutel mit Sina gefticket / beren eis ner mit velen meffiagenen singen wunbetlich vers jeret iff.

79 Sin Indianifoser Parafoll aus Siofre und mit Debl getrád diten feibenen \$apier febr fauber gemadoet.

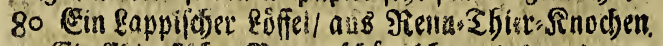

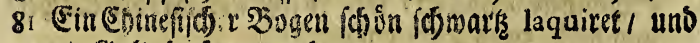
mis Golo faiber eingeleiset.

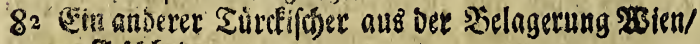
von fidobrin.

83. Ein Prifma vou fojonem Eryftall.

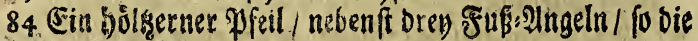
Huiliten bey Sernau ausgsfreut.

85 (Sin 2bauß in Sinz von Tbro Majeftat Des Runigs von Breuffer Geficite.

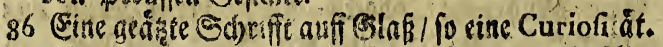

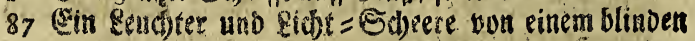
Bauer bay Strabsurg/ aus finflic) jufanimenge-

fúgten Scolige artia gemadhet.

88 (a) Ein Microfcopium mit Beffelle von SDRefing.

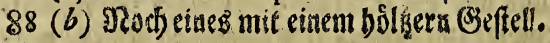

89 Cin 23renn: Spiegel in Diameter von $\frac{3}{4}$ S $\$$ uff mit eimern Colleetuv Bilalen nach ber fererren von Sajtrm baujin Invention mit jugegurigen Beflitlin. 


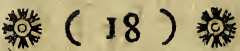

bodif / uth s. Fun lang/ burch . S. D. Scheuchzero. I IO Nod) eine won ber Eani/d) aft Euggenburg/ab eodem. II Tab. I. Lucius Antediluvianus. in Siupffer/o Mufeo Scheuchz.

I12 গoci) 16 . Stucic auff einem Supfier ans dem Mufeo.

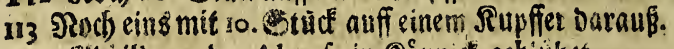
II4 2Wbilloung oer Aloe fo in Ripnide gebitibet.

us $16 b i l b u n g$ Des Cerei PeruvianiSpinofi jo in Eenden gebluibet.

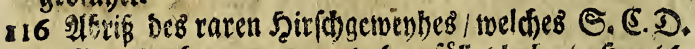
justandenburg Anno 1696 . gefállet baben. Şat 66. Ěnoen.

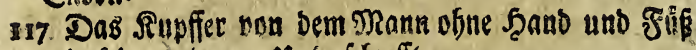
mit feiner eigenen unterfdfr:ffit.

218 Rupfifer von ciner (1Ribgeburtf) von 2 . Rinbern \$Beib;

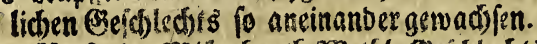

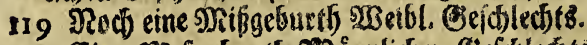

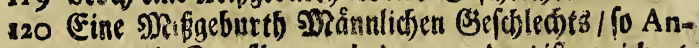
no I697 in Etrafburg gebobren worben/ iff gessidjet.

121 Diefe in Profil gcteidjnet.

122 (Ein Ralb mit 2 . Sibpfifen.

123 (Ein Niel mit 2. Mispfen.

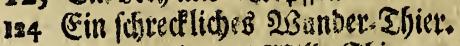

125 Ein ungebeures sistlbs Zhier.

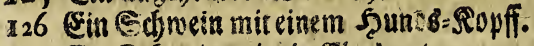

127 Ein Sdbwin wis cis Elepbant.

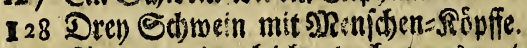

129 ग2ud uren Dergleidjes bed etwas unter(a)ieven.

130 Sdivein mit ein m Eulen=Sopff uno Doppelten \&eib.

13x Ein Doppelter Scaafe.

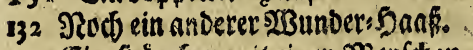

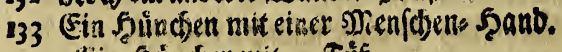

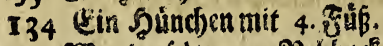

135 MBunderfelsamer \$Rehbot.

136 (Gin Dd) B mit unfurtnlidfen Fúifen.

137 Etae Saube mit Drey Fliegel. 


\section{Bon $\mathfrak{B a d}) \mathfrak{s}$ poffirte und von Gips gegofiene Sadben.}

I Das Portraic bes Reinigs 23 ilfelms in Engelland.

2 Bon Rónig Frieberid) in \$reuffen.

3 Dbgebadfen Rúnigs Ëemablin.

4 SBon Eburfírft Sieorg 2Bilbelm von Sanober.

5 Des Eron-prinken von \$renfen.

$\checkmark$ Der Erb:Princelfin an Eaffel vermåflt.

7 Der Egurfitrfin Expbia von Sanover.

- Carl \$filips Efurfürfen bon Sanover.

9 Sionigs \&ubewigs XIV. vou frandereic.

1o Earl bes XII. Rónigs von Edjweden.

II Carl bes XI. dito.

12 Ulrica Elennora Der Siduigin.

13 Chriltina Der Rönigin.

14 Euberwig willbelms Pringen yan \$aben.

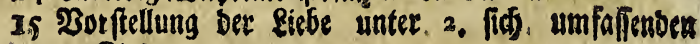
Rimbern.

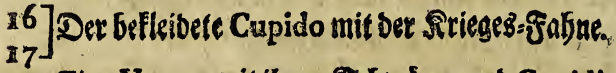

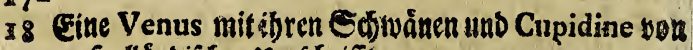
Şollånbifa)er Lumichriffit.

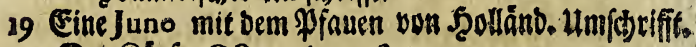

20 Der Siñfer Oetav. Auguet.

2I Vitellius,

22 Nero.

23. Julius Gafar.

24 Ludovicus Magnus Franc. Rex.

25 Carolus Secundus Magnæe Britan, Eranc, \& H Ho bernie Rex.

26 Cin beey boppelt \$2Bapes.

27 ?

98 4. Unfeltannfe alte Portrait. Diefe nit Den boxis 39 gen find alle von Gips. 30) 


\section{漛 ( 21$)$ 耀}

31 Det Reib von Lauconr. pronfitt.

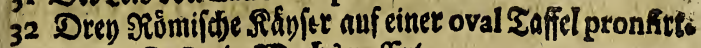

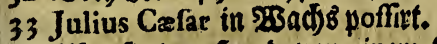

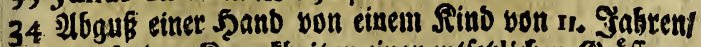

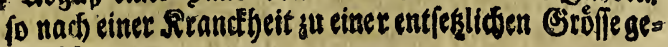
madisen.

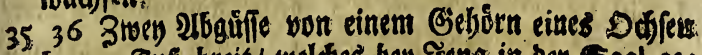
bey 4. Fußs breit/ mellojes bey) Jena in ber Saal ges fuuber/ Des Original if bier/ uno wieb aufi 100. Sbl. zeftimiret.

\section{Dritter Sunung. Erfter UnterfabeiD}

Darinnen von

Ibteren / Bogeln / Sifden uno Sd)langen / $x$. $x$.

Neebff ibrens Sycilem/ audes.

Einigen Zoophytis und Cruftaceis. aus Der See alles trudeen conferviret. allerfand auffbefiolten inerben.

Necff einem?tnbange/barinnen

Atherband Artten von Steinen Det Menichen uno SGgere enthalten.

I.

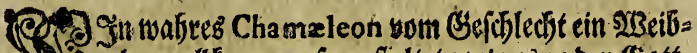

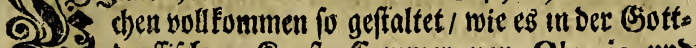
Dorfifichen Sunft = Cammier yon Oleario umb anbern gejeidjet. 


\section{整 ( 22$)$ 耀}

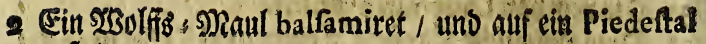
geffellet.

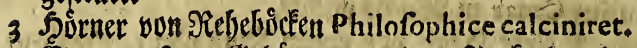

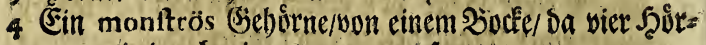
ner artig burds einander gewad, fen.

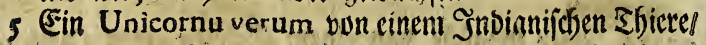

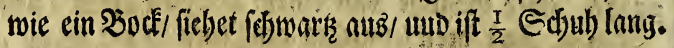

6 Ein Soun uon cinem jungen Najen - Sbiete/ if vorne nod) ftumpff / obngefelir 4 zoll boch.

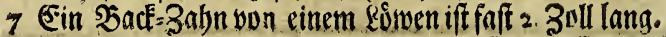

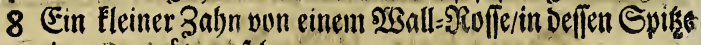
eine cavirät - on felesi

9 Ein Fellnen von Tunguiniforen Morcho.

jo Zwen andere von Mofrrwit foten.

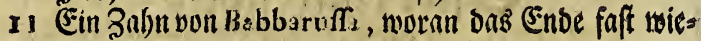
berumb in fein Principium getwachjen.

12 Ein Glásgen fómen = Fett / aus Capo bonæ fpei.

13 Ein Şauer von jungen Esber.

I4 Einige 5raare/atiz ein 8 alten Elephanten Ed dwantser fo diff / wie Sindfaden.

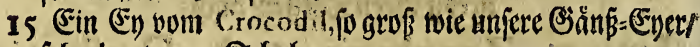
felbr bart won Edbalen.

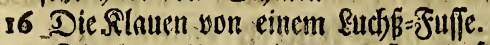

17 Die Sacculi von einem Mofcowirifben Sieber.

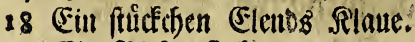

19 (Fin sieber=3abir.

20. 3weene ungemeine grofle Stadgeln/ bon Stachel= Edbweinen.

21 Eine gruffe ablánge Americanifobe Subild= Stứte mit 13. Edilioen.

22 Eben Deraleiden an Biviffe uns Farben.

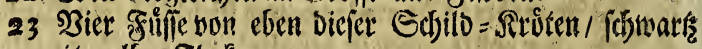
mit nelben Slecten.

242526 Drey unterfdiedene 2rtben/ wobl poliertet Edjilo = Nrotent. 


\section{絜 (23) 粼}

37 Sine Sdjale bon einer befondern sint / gant weiffer Schild= Rruten.

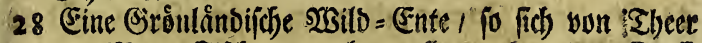
unv SReer = Fiichen zu nebren pfiegen / bat einen Siopfif als ein \$apagoy

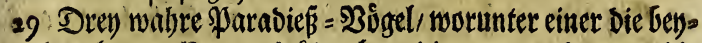
Den langen Extremirâten bat / Die man vor bem yor bie Seine gel)alten/ alle Drey baben fǘffe.

30 (Ein En von fleinem Yogel / nidjt viel fleiner / als ein Gants=-Ey / Da peine \$nttter nicht gróffer als eine fleine Scenne ift.

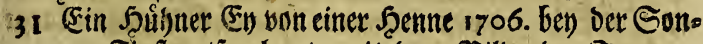

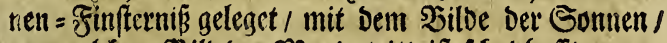

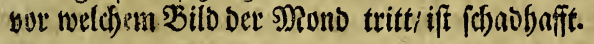

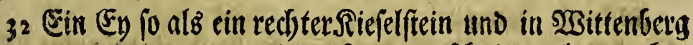
Anno 1712 aus ciner Scenme gefbjuitten laut Dabey lienenden Sirieffen.

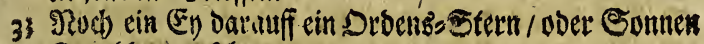
E.rofilen gu felyen.

34 Eir Ify/ Dariunen nod sin anber (Ey Gu feflen.

35 (a) Ein Eiv Darnuf cine Estonedten Rénie.

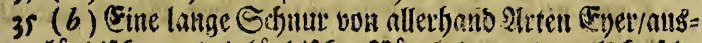

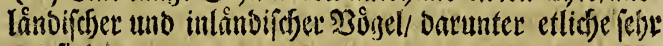
tine fints.

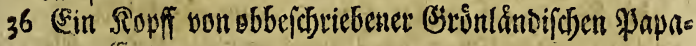
goy $=($ ente.

37 (a) Ein Sippfi und Etfinabel bon Vogel Tattau voer \$ieffer Freffer.

37 (b) Sdjón polietter Sdjnabel bavan.

38 Eine Silate von einem grofin 2 toler।

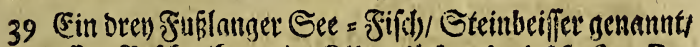
Deffen sef(d) reifung in Oligarii Jacobai, Mufao Regis Danixgefimben wirb / wiro auci) Lupus Indixe Orientalis genannt.

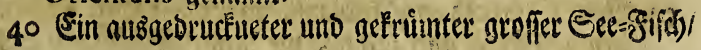




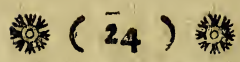

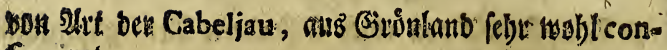
ferviret.

41 Ein grofer Humer mit einem 2ufter = Nefte nuff bent Rlicter.

\$ Siwey Humer etwas fleiner.

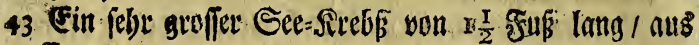
Engella 0 .

44. Eine Edreere bon einem anvern in ver Einge faft cinen Fus baberso.

45 eriapus Ceri won einer ungemeinen Diffe unt Brifife. 46 Der Siacjen vontifíce Carchar ir mil 4.2ietibenzấlonen.

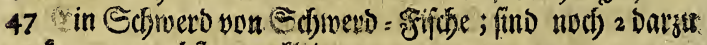

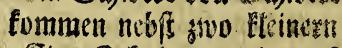

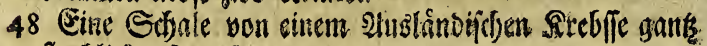
ffach)lidft if weê.

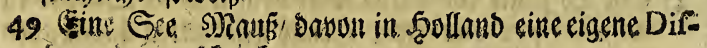
iertation Befibrieben.

so Eiu Riefer von Delphin.

SI Ein curës: flein Unicornu marinum yon einem utrs gelioijunen jungen Narval.

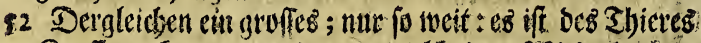
Sopf verborgen / und vermittelit. Der sisieberbaden. welche artin zufeben / befofitiget.

13 Cime gruffe \$erlen = Matutter/ auf beren andarn Seite

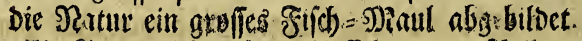

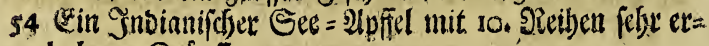
babener Sinopfen.

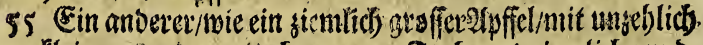
fle inen Eminentiis braun bon garbe uns gtemlid) tumb.

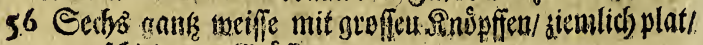
unteríbiedener Bsuffe

$\$ 7$ Ein etwas fleineter braunet:

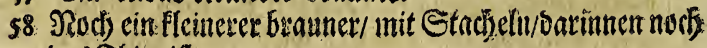
Das Shier iff.

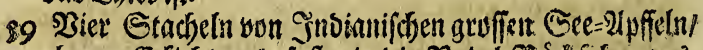

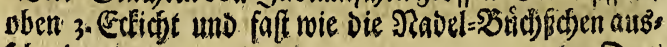
Scheno. oo Dien 


\section{蹸 (25) 橉}

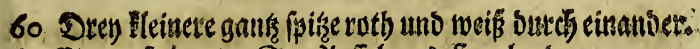
6. Ein verffeinceter Sie=2ipffel aus Engelant.

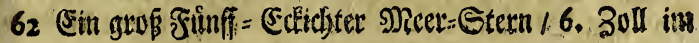
Diametro.:

63 Fin Heinerer an anberet 2 (rt.

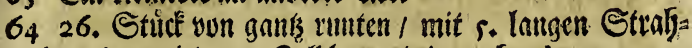
len / Deren jeber 2. Zull lang/einige gebrodden.

65 Gin gant fleitier youn 4. Strablen.

66 Einige See = शnabel.

67 Einge idjone Enthalia majoza eint Esjnut.

68 (a) Invere fofone Enthalia minora eine Sdjutr.

68 (b) Eine -\$arthen Byzant if ber \$lattem.

69 Ein fo genantet See $=$ Ball.

70 Eine fobone ganf runte \$perle / wire eine gute Wifolens Situgel nach ciner ftartét inundation bey. Oclamünde auf vent Selbe gefunden / ber fedffec Theil fo reiff/, if

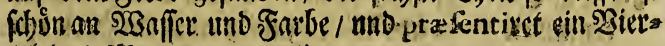
tel Des Mion en gar artig.

71 Dreigig Etuef groffer Orientaliffen \$erten/theils

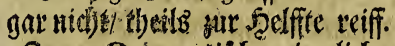

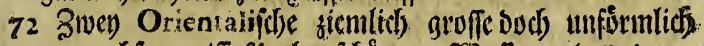

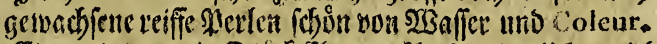

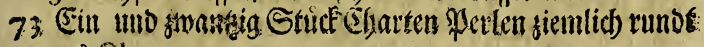
aแถ China.

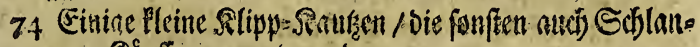
gen = Siopffe genaunt werbin.

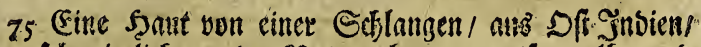
fobr fierlich yon Der Natur / braun / weis / gelb uno

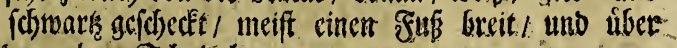
irenselen Selluly lang.

26 Eine Epidermis bon einer Sdilangen/ wo fie fellet

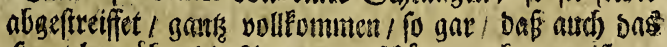
Shautchen ifber bie 2lugen gar fofoun ju erfennen iff.

77 Eine Italixenifore atsigcontefinete Dtter in cinanoet gefdolungen.

$$
\text { 5) \$5 78 }
$$




\section{徆 $(26)$ 赫}

78 Fin Scheledon von einer ferte langen Amerieaniiden Sdflangen/ von 268. Gelentêten viorer Verrebris. 79 Ein Stúd Cranium von einet Egpptifoben Mumie 80 (Sine Aameticanifde groffe Sheurofect.

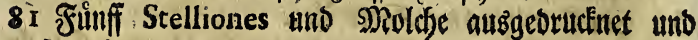
- Laccirt.

82 2. Sthlangen auf foldite 2 tet.

83 (Sin See=Sild) voller weiffer Stadjeln / See = Taube genannt.

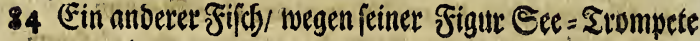
genanut.

8s Ein Crocodill.

86 Fin rater See=Nrebs obne Sdieetert.

37 Monftrofe Sitebs = Sdjecten.

88 Ein unreiffer Coloquinten Ipffel.

89 Sin Sthrober ober Frter - Wrutm mit cinem langen

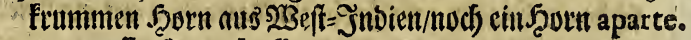
902. groffe Etrauk = einer.

91 Eine fo genanote Edjlangen Erone.

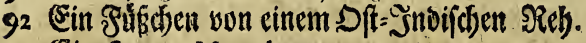

93 Ein Lapis Mnatis.

94 3wen fanbere Sirabben = Stbalen.

95 Dren Stidf polirte Sdjildfrot Sdjalen.

96 Ein Dens hypopodamus.

97 Ein grus Rhinoceros . Suth.

98 Der Riunigg= Bogel.

\section{Sintlang}

Berfdiedener Steine aus Men= foben uno beren sbieren.

I.

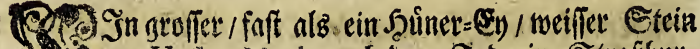
Q10. exVetica Maris nad Dem Tode im Strafbur= Q. then angewadsen gewejen. 2 Fin 


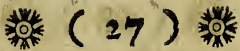

- Cin Etein ex Vefica Bilaria foemine finto jiver gemes

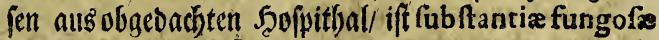
uno gelb bratn/ foft figure Cob cxe.

3 awey Hleinere rothe Steine meldbe neloft bentigenden weifien von einem Nephricico nbgetvieben albier.

4 Eechs ano deenpig Steine / cx Vefica bitaria foemine, finb 42. gémefen / alle foft nusmentio / wie Oriental.

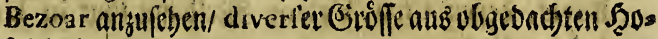
ipitisal.

5 Ein Etein/ben biit ex Pulmonibus Ifterici Lethargo \& Apoplexia morientis eines Ect)eiber = Gefeftlen 1702. Den 9. Novembr. Kernus gejehnitten / uno funs Den fich viele iartuner mie Ş̧irfe/ nebft ançegangener funge.

6 Ein aroffer Stein won ciner Frau aus Sinfterwalba/

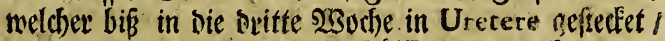

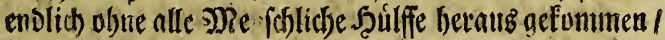
if figuritct als eine Teule unten ppitigis $2 \frac{1}{2}$ Zull bochl und s. breit.

7 Eit nroffe quantirât Fieren = Cteine ex homine, 8 Dren fiemlicf) groffe Steine ex Rhenibus An. 1694: . Diefde 1. 2. Sctupl 2.1. Scrupl, 3. 13. Gran.

9 Etcine ex Vefica bilaria Dresda ex Mare 1694. ro Rezoar mulinum, ober ein Stile von einem Etein/ po in einem sicul = Efel eentacbjen.

xI Ein Stud bon einem Etcin / fo in enem Pferoe ge= wachín. S. Bezoar Equinum, th

12 (Ein fiemlich aroffer Occidental. Bezoar, griultich.

13 (Fin Dundfelbrnumer aenud)ter Bezoar.

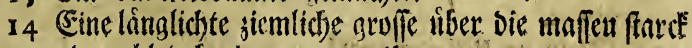
- uno mobltriecterbe xgagropila.

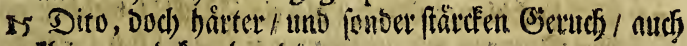
fleiner uns fugeltrinot:

I6 Ginige Fenertwhe Sturfe/ won einem Stein ex Vefica tilaria Animalis.

17 Gin groffer und zelyen fleine Steine ex Rhenibus Bo. 


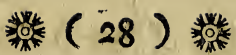

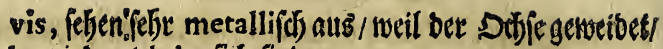
ba biel acidula fich finben.

18 Ilus obgebadjten Dobjens = 2jlaje cine grofe Quan-

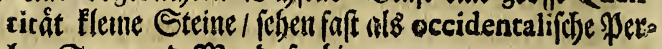
len/ Sonn uni Mions farbig.

19 Ein Ovaler fonneeweiffer Stein Umbilico fuillo. 20 Fragmenta cines grofien Steins ex Vefica bilaris Bovis, Deffen Char. Valent. Mieloung thut in Difp. d. Lap. Porcino.

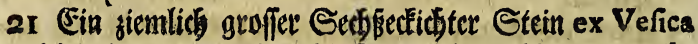
biliaria Bovis autswenbig braun / imwendig gan/zrotb. 22 Ein Lapis Porcinus ex Suecia.

23 Ein dito gank meifier.

24 श2ods ein andeter.

25 Ein ziemlith groffer Stcin / ex Vefica Urinaria Csnis per longum tempus dyffuria laborantis, wel= (ber nebft noci) 2. groffern / und vielen gants fleinern Die Veficam ganfs extenditet / uno ben Scund umbs

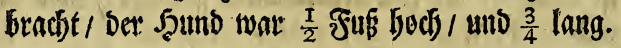

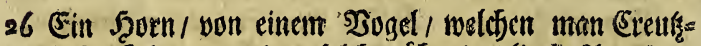
Sdjnabel nennt / Dem foldbes hiter bas lindele 2litge bers.

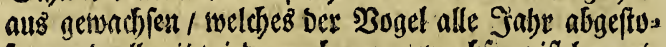
fen / uns allescit wieberumb nes gerwachjen/ iff lang ets iiber ein Drittel Zoll.

27 Petra del Parco, welder aus ber Ballen = \$la la beo per Strchel Scjweine aus der Inful Madagafear fom= met / uno fehe medicinalifob iff/ auch beswegen theuer bezinglet wirb.

28 Lapides 1. Lapilli ex Cerebro Pifcis Indici incogniti.

29 Ettein ex Pifce manati.

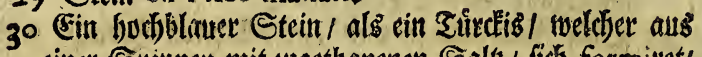
ciner Epiunen nit jugethanenen Sals. fiti) formitet/

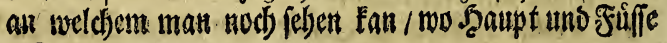
Beftantern. 


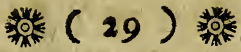

31 Ein greffer Stein/ fefrumberlif geffalt if bier bey cinem Nerfofen ausgechnitten.

32 Ein geller Nieren = Stein.

33 Ein Stick yon einem Stein aus bem SRngen cines Nerbes.

34 Einige Sects = Stcine.

35 (Einige Sour $(d)=$ Cteine.

36 Eine Copia yon einem Stein $y$ weldser per inferiora won einen Miann bey 60. Iabren gegangen' / babey nod)

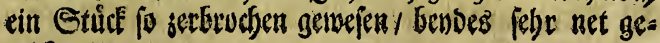
seidsnet.

\section{Dritter Stomung. 3imenter Unterf(d)ied Darinnen von 2llerband uno meiftens}

\section{Indianifden I N SECTIS,} Efllide bundert Stùd" gesellet weroen.

(3egentoantige IN S ECT A fins alle tructen balfamiret, und werden in 36. runben $\mathfrak{b}$ Łáltuniffen set: mabret/als:

No. I. 9. Stúck.

O92seene braune 3immet farbene 3weyfalter (Papi-

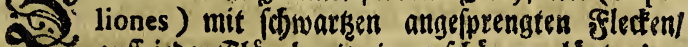

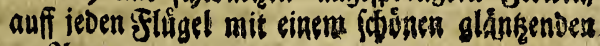
\$rauen-2lugen. 


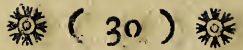

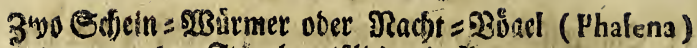
Deren vorber Sflügel weifflich mit Siraun gemarmett

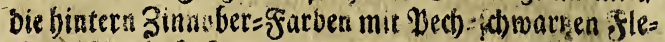

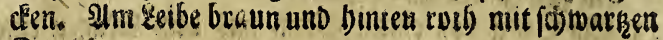
Stitiden.

3100 andere Siller farbente mit idmartzen Bârtgen uno Fusifer.

Bier bodblane fleine Rấfergen.

$$
\text { No. II. 9. Stúcl. }
$$

(Ein Strob)=fakbener groffer mit (d)wark uno blau mars moritter Zmenfalter voer Sommer - \$ogel/ anfi ben bintern fFlúgeln mit cinem Pfauenseduge und langen Extremitáten.

Drey andere gants gelbe/anfi beren jeben fliggel ein groffer Orange farbener $P$ unet $3 u$ fellen.

Cin anberer braun= rother mit gelleen und fojwarken fles den / am Raabe ber flígel braun uno fifmais eita gefafet.

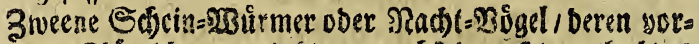
Der Flügel brana/ bie bintern nebfi Dem Sintertfell Des Eeribes હolo=glăngend.

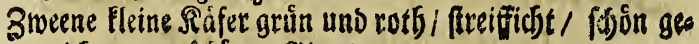

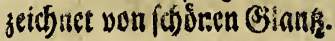

$$
\text { No. III. II, Stůcé. }
$$

(Ein gan brauner Sommer = Bogel $/$ auff befien yorber

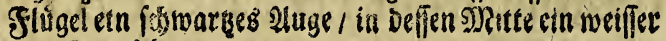
Punct ju fefen.

Bmeene andere b: aune/ mit gelben und fob) uno blau uno idiswat se eingefaften Ffliguln. 3ween gelbe 3menfalter febe zart rothe eingefaffet / auff

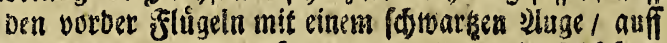
Den bintern mit einem braunen/ oarinken ein gleids fam bun S̈uld gemadtetes Slecten zu feben.

Bweene andere/ beren einer bunfelebraun mit weiffen fllecén / bee anderne auff Den siuiten liegend / weís mif fow warken Reern. 


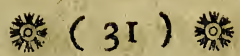

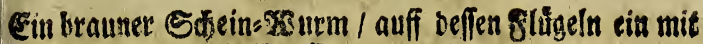

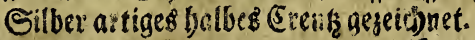

Fin anderer von brann/gelb und weif artig marmotiret. Zwerne flinere/ Deren einer auff 2 (c) =grau mit fdjwars

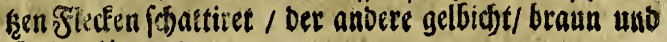

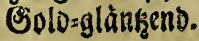

$$
\text { No. IV. I3. Stud. }
$$

Ein Pleiner rarer Eonmar = Bogel/ beffen vorber f̧liggel gans lotwars mit Eremofinerothen Fletén / bie bin. tern Eeemofin = roth mit fonmarken fliden anges fprenger.

3weene braune ober 3immet farbene grofie Echein.s3air. mer mit idjwarksen 2toern marmoriret / beren Sflugel artig ausis efterbet.

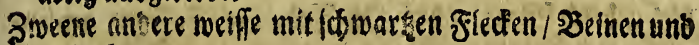
Bàrtben.

awiene fleinere gelbe mit branuen ffleden I Beren binter

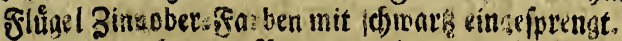

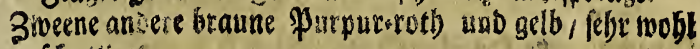
(i)attice?

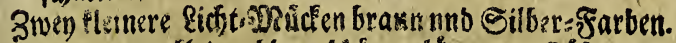

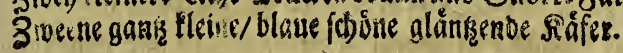

No. V, 7. Etuick.

(5in grofler Amboinifher 3menfalter / mit 4. Durowfids)

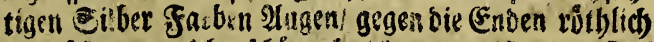
und (o)wars/ febr idfón |chattiret/ meiftens s. 3oll lang.

Ein lanaer $23 u r m$ mit viel bunbert Ş́ffen / aus Jnbien 4. Zolllang.

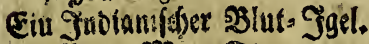

Ein fleiner MR :er-Etern.

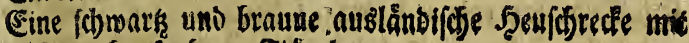
3innuber farbenen oflitineln. 3 mey Hleine braune Rốfergen. 


\section{嗮 $(32)$ 新 \\ No, VI. I3, Stuck.}

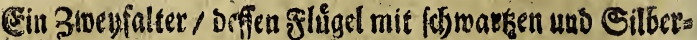

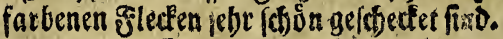

(Ein anderer auff andere weife mit idjwars / weip uno braun auff Selbichaitiret.

Bmeene gellblicite braun fojattitfe. Fleirere.

Sweene werffe mit fow wargen uno gelben Gleden.

3weene bellbraune mit bundelbraus artig / langen \$Bärtbeu.

SBire Silber-farbige mit M(o)-grau / broun und forwars (क)attiret.

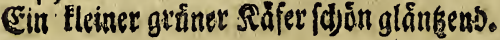

$$
\text { No. VII, 3. Etůd. }
$$

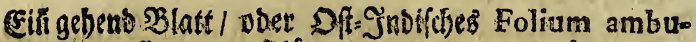

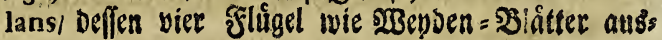
reben.

Gin groffer Råfer mit cinem Hrummen şarn auff Der : Rafer.

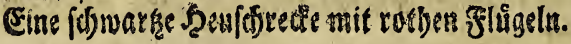

\section{No, VIII. I3. Strick.}

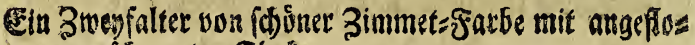
genen (d) warken flecten.

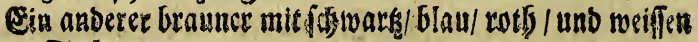
gitedien.

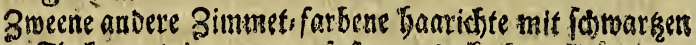
Glecten no brata eingefaften nusgetéterben Glugeln. Cla anderer Strob farbener mit bell und Dundelbraunet Streiden gemarmeit.

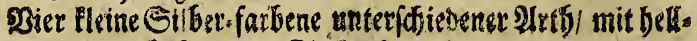
und bundel Graunen Fleden posattiet.

CEin Silberfartener ins Graue fallewderfleiner 3mevfal

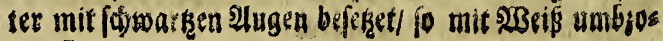
gen fino.

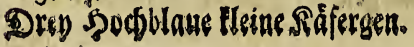




\section{称 $(33)$ )}

No. IX. 2. Sturt:

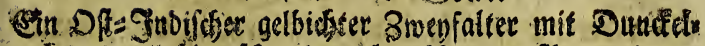

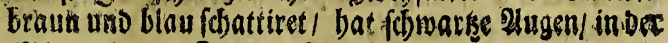
Mitte ein meifier Punet su spen.

Cine grimeibeurchrecte.

\section{No. X. 5. Stuif.}

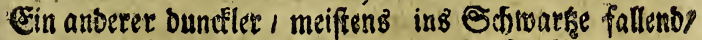
mit weifen Gtecten / und mit Zian ber Muneten ber

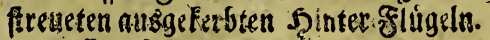

Eine groffe grine Şeufforecteten.

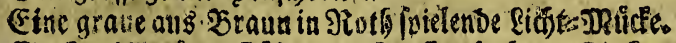

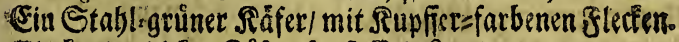

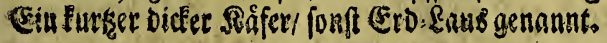

\section{No. XI.}

Cine grofie aublân ichmarc icjattiret.

\section{No. XII. 4. Stuck.}

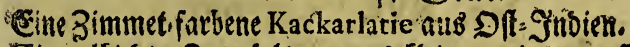
Ein gelbichter Zawenfalter etwas fleinet wie ber erfe/ in Der erffen Capful.

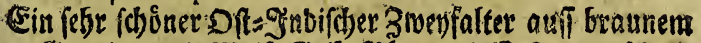

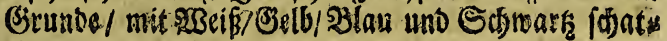
tiret.

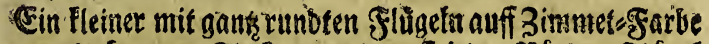

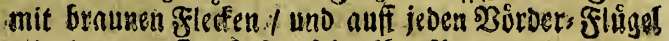
mit einem groffen Sditwefelegelben Nlingeo

\section{No XIII. 11. Stül.}

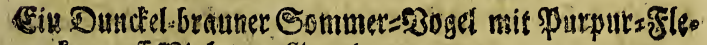
cien antiff Bioletten. (jirunde.

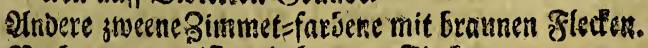
2Rod) gneente meiffe mit braunen Flectent.

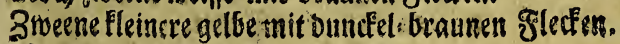

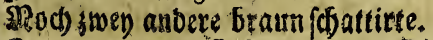

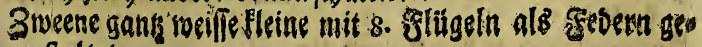
frallet: 


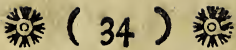 \\ No. XIV. 9. Stůct.}

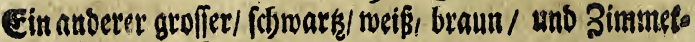
farbanis wit Saanen.

3wicene ganh benune mit Eidften flecteren.

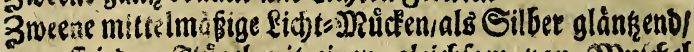

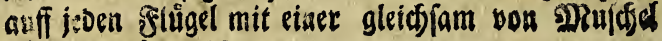
(3). 10 gemad)ten Marque.

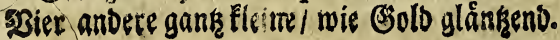

$$
\text { No. XV. 5. Stůce. }
$$

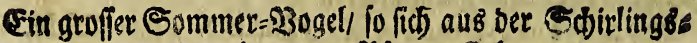

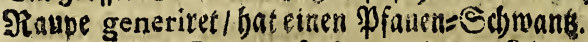

Bmeene andire Bemmet:farbene mit Dunctel = braun unb

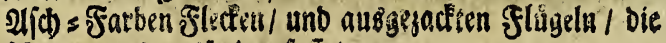
iómark und weí e eingefallet.

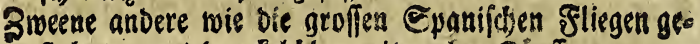
fraltet/ gan bundelebblau mit rotber Ripffen.

\section{No. XVI. 5. Etúd.}

Cin groffer breiter Sommer = Bogel 5. unb ein ljal5 Zoll lang / aus Amboina, nit buncelel biaunen Strnofen/ uno me iffer folectern auff bells braunen Birunbe.

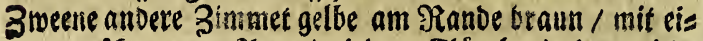
nem fobmarken 2luge in jebem flligel / in beren jeve zwen weiffe surcte.

Zwoene anvere ganks weiffen neblt stween weiß und braus (c)attivten.

\section{No. XVII. 10. Stuict.}

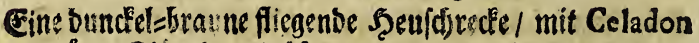

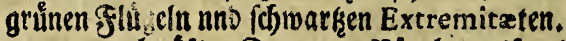

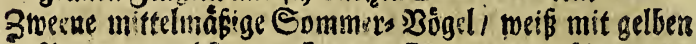

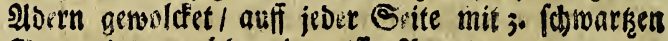
2ügew in icrun jeben vin weiffer Punct.

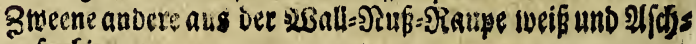
fa bia. Sowerne fleine bell-gelbe mit augen. 


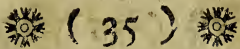

Cin bergleidyen busdel braun mit 12. Orange. Couleur filetern/ bergleidien Eilbe:-fatbig.

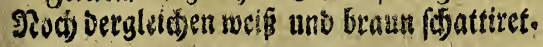

\section{No XVIII. 8. Etüá.}

Ein groffer nus der efoinlings= SRaupe generirter Som. mer:=3oacl mit pern oben angeseigten meiftentoeils gleid) / miem ofl in cima $B$ different.

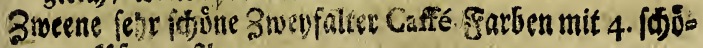
nex Pfauersolingen.

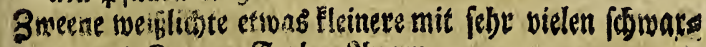
hen uni Orange=Farban Plugen.

Drin fleinere, weis und fojwaris fofattitte.

\section{No. XIX. 9. Etricf.}

Drey fraune fefre ithoin fojattitte Sammers:Bögel unter einander etwas unteridaieden einer if haaricbt.

aroene braune/ Orange/ blaue/ weíp uno / fowark artig

marm rire antere.

Bwo floine gruine Flliget.

Ein Fleiner Scjein-3ogel.

$$
\text { No. XX. 9. Stuid. }
$$

Ein arofier Sommers = Bogel braun / mit Strob-Sartien Slecfen und vier PFauen. Auger.

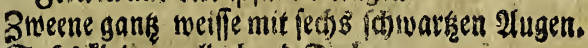
Eedjo Pleinere allerbano Garben.

\section{No. XXI. 9. Etuce.}

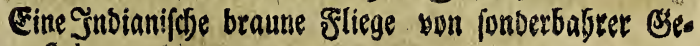
fialt.

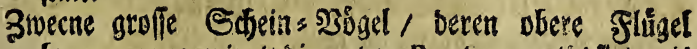
Graun marmorizet/ bie untern Ponleaustots/ fino mis

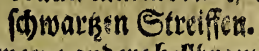

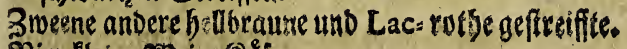

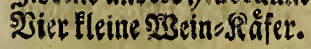

$$
\text { No, XXII, 9. Strice. }
$$

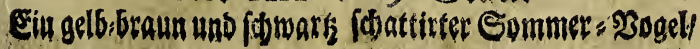

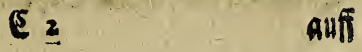




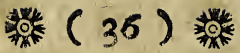

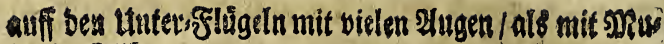
fôteln Silber.

Sweene andere weißs/ braun und gelbe.

Zwiene Bold. farbige mit weeffen Flưgeln / morauff fidmarge uab Bolo farbene Stedede gefiprenget.

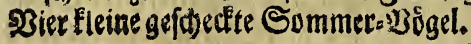

$$
\text { No. XXIIr. II. Stuct. }
$$

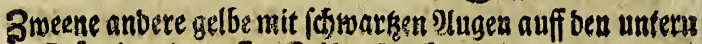
F̧túgeln mit atofien Silber- flecten.

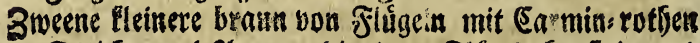

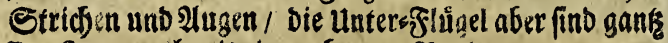

Ponfeau - roth mit einem brauen $\Re$ ande.

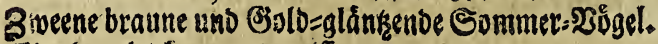
Cine Dergleidjen ganş weiffe.

Brosene Elsinere gefofiedte.

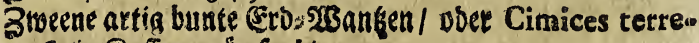
ftres Duffe:=grin farbig.

$$
\text { No. XXIV. 9. Stůce. }
$$

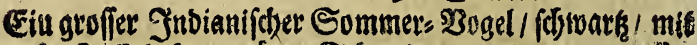
bod Celadon griner Sdjattivung uno gans weilfen Extremiraten / fifre fofón.

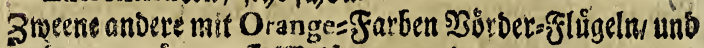
Ginten grim auf 533 is marmorirt.

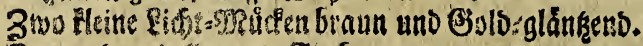
Zwo andere betler som farbe.

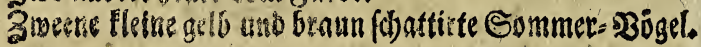

$$
\text { No, XXV. 7. Stuld? }
$$

Cine ganh gelbe simlid groffe \&idjt shicfe.

3meene anowe aelbe mim braune.

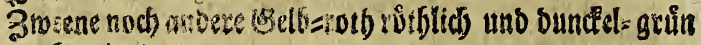
idnottiret.

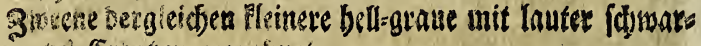
geti Ereufen gescicinat.

$$
\text { No. XXVI. 9. Stůcé. }
$$

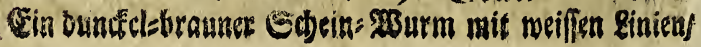




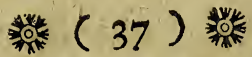

mit Ponfeau $\leq$ rotbert Fluggeln uno Eeibe/ barauff $\$$ echs

fotwarke Stecten geftreuet.

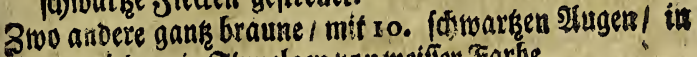
Deren jeben ein Sippelgen bou weiffer Jararbe.

(5in Orange-farbener Zmeyfalter mí braunen Extremi-

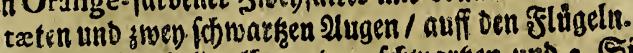

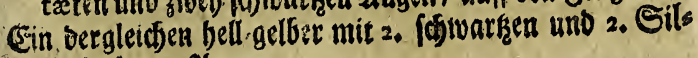
berfarbenen 2Uagen.

SBier Flsine weifs=braune und Silters farbige sidjts macicerer.

No. XXVII. 3. Ctück.

Ein fell 5rauner Eommer=\$3ogel mit iomarksen flecten] und fillornen slugen auff ben Fliggeln. Bweene lleinere witfe mit fleinen Sleffen.

\section{No, XXVIII, I2, Stüce.}

Ein Gbolofarbiger Sommers Bogal / mit weiffen Fligeln/ baranffídmarke und Godo farbene \$uncte geffreuet.

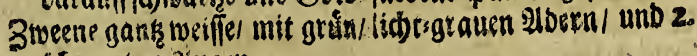

fobracken 2ugen.

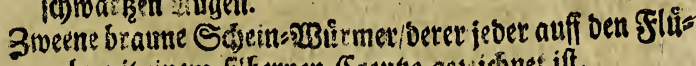

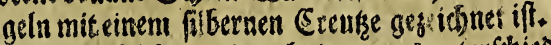
Breene beraleidsen/ wiewob) in etmôs unter(chieden. 3woene fleinere: brana icjattir:e.

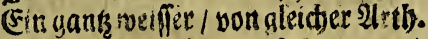

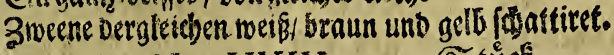

\section{No XXIX 25. Stude.}

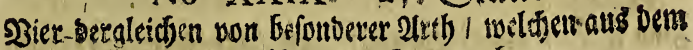

Sdimanthe 3.lange fow warke Saare geber.

כier grưte Râfer.

Sुier artige Ráfer idswar uno gelbe geffreifiet.

Sier De:gleidjen lange/ braune / ponft wie Spanifde Glies

gerrogfaltet.

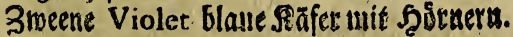

Zmeene fleine firegenoe Scorpionea.

Bier ganş Fleine gruine Siáfergen.

Cin grin uno braun idjattirter Săfet mit Scumem.

(5) 3

No. 


\section{橉 $(38)$ 兟}

No. XXX. I8. Stuct.

Cine attslantoifobe Endert.

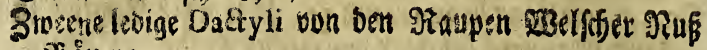

ЂBătนate.

2ct t inhere vin anbern Raupen.

3wo flene Libelta / ther St)ielfolle artig geffal'set.

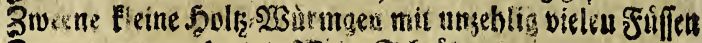

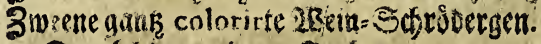

Derglicten anderat Farben.

Eiae lange Erb:23anth.

No. XXXr. 6, Etuide.

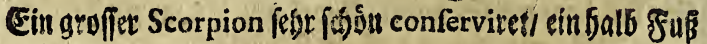
lang.

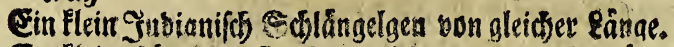

E:a Picia jủgelgen Quaianumbi geverinet/ mit gruin uno

Gulb farben Febeen / welds) B vom Than auf Den 3 lus men lebet । und Den Enfit Daraue fauget.

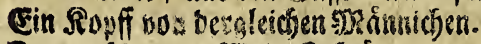

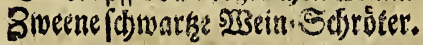

$$
\text { No, XXXII, 13. Stuce. }
$$

Swo groffe Şeufejtrecten von beriofebener 2arth.

Zmo vergleids $n$ Eleiners.

Ficunilleine Siffer rotb/grin/ blaw/ uno getbe gefobetet.

$$
\text { No. XXX111. 17. Stud }
$$

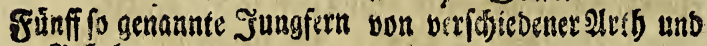
Geitalt.

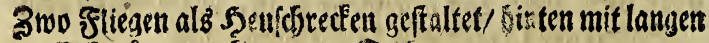

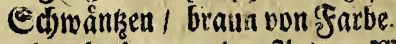

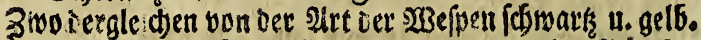

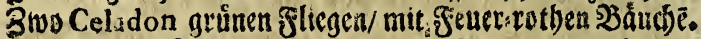
Bier Fleine Sơfer/ Celadon, Violer, braun uno gelb voa Farber.

3100 Tattil= Rern / won Heinen Raupen.

No, XXX!V, 10. Etuef.

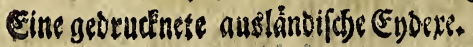




\section{橉 $(29)$ 耀}

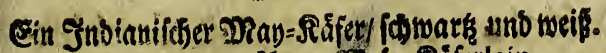

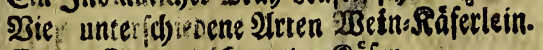

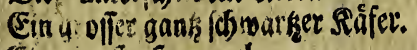
Eine grolle funmmel. Eine gaus itswarkelan se Cimex terreftris. Eine geibe und iffmarge Paupe.

\section{No. XXXV. 13. Stuid.}

Ein Fleiner Jrtoi (d)er Eto = Erocoboil.

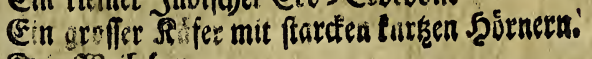

Gin 2Bcibden.

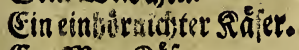

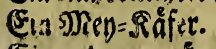

Ein anberer grün und roth glängetbete.

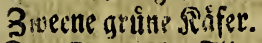

3 woo Spantiche Estiegen.

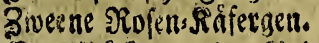

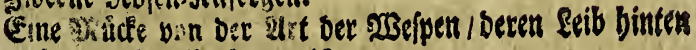

baib totb ball bidwarg ift.

\section{No. XXXVI. T4 Stůce}

Ein Snbiunifder Kaufnoffus 4 3otl lang.

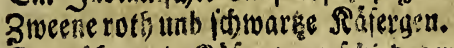

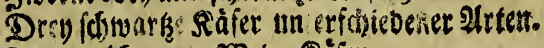

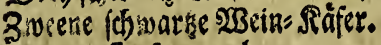

Eine gre fife Summel.

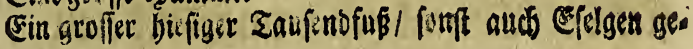
Eannt.

3no befordere Cimices terreftres.

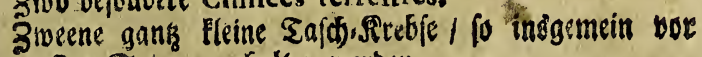

See:Sp numen gejofilten werben.

\section{Dierdte Dronung}

Darinnen rare Saden aus der See, als nemlid) yon $\mathfrak{A l l}$ erband Arten

$$
\text { (5 } 4
$$




\section{裆 $(40)$ 耀}

Murfoth / einfadsen uno doubletten, gefdranbetn uno glatten úber andertbalb Saufeno Studfe befidries ben werden. Sobbf cinem doppels ten alnbange von vielen fetse racen Stutcen Bernftein mit differentent Darim befindidoen Infectis tuno als Ierband Arten curieufer Sel= (Ges wád)

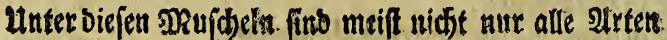
bie ber feer Rumphius in ber Amboiniffen Runflis Sammer/ und ber Jefuit Bonanus. beldjrieben/ zu finden; fordern aud nod) viele anbere rare fo in menig Cabinet-

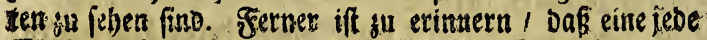

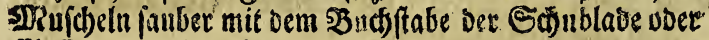
Claffe uno auch) zugleiố mit Der Nummer Des Catalog bemerdfet ift. Iftuds ift nods ein Bateiniforer Catalogus vorbanben ber gleitbfals mit Citirung Des Bonani und

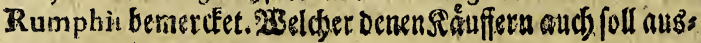
geantwurtit merber.

(Erfte Sdbublase/darimnen meis feno sie Ginfade und angedretheten Siufdeln.

\section{Sub Signo A.}

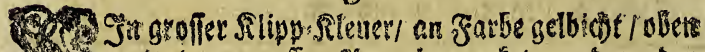

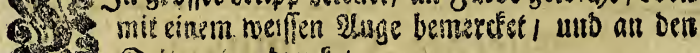
2018 siler engetruciet.

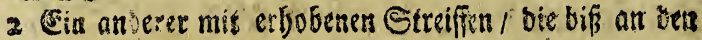

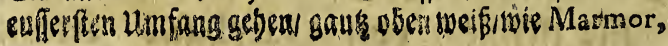
aus? 


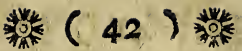

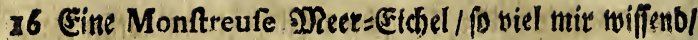
bon feinem Auctore bejdrieb $\mathfrak{n}$, befely:t aus 8 . By-

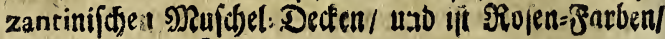
gelbidst uno we ef.

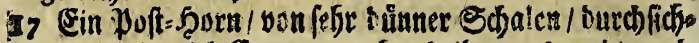
tig uno in viel Cammern abgetbrilet ausweredis gel: bidit inwendig Perlen=?atben.

18 Drey andere bon verfobiedener Grifile

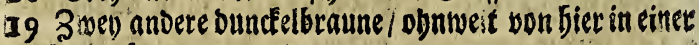
Sire gefun:en.

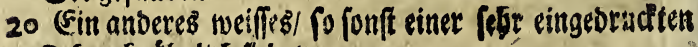
(Sd) edf: ábnlidy fiebet.

21 En anberes traun rotbes fleineres/ meldece fich sulest

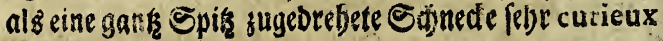
cribiget.

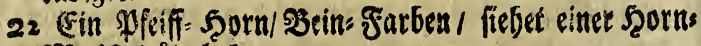

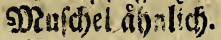

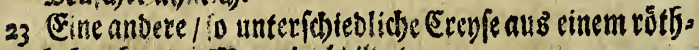

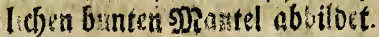

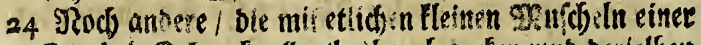

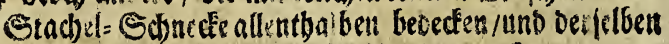
allenthalber ein monit eufes 2 (n eb $n$ madten.

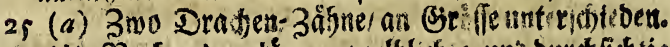

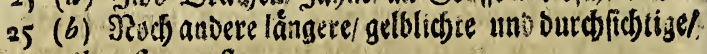
quibus femen fpargunt.

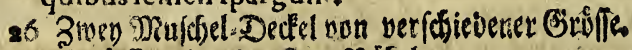

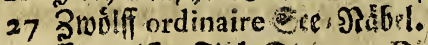

28 Einneifiter ffi dy Eken 29 Dito.

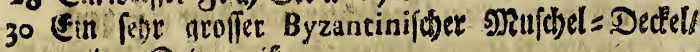
an einer Eetic weif.

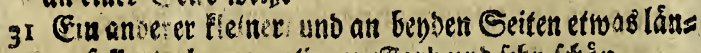
ger fallend b aun rotis you gsarb uno fegre fajoun.

32 Ein anderer $\mathrm{O}$ ccidentalificter gelfer $/$ boa anbern dor Ftaur nach febr unterfos eden.

33 3rou braunc und an enferffin umfange geferbet.

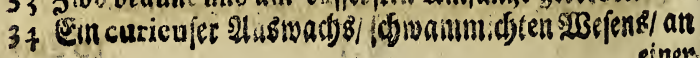
einet. 


\section{粼 (43) 䊁}

einer doublette bie fonft Mufculngenennet wetbetto 35 Ene Defter Doublette allentbaltien uneben / Daran

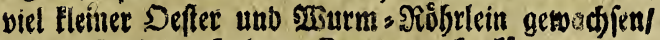
Dakero fie einem Scabnene fiamme nafe fommt / tut

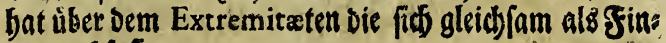
get umbfafien.

36 Ein gank gelber glatter (s)aper.

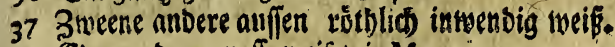

38 Eine andere oroffe weif wie Marmor.

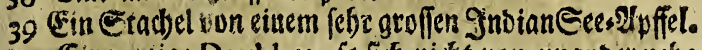
40 Eine artige Doublette fo fin niøt yon einander nefs men láflet.

4I Eiae Duffer=Doublette/ baran oben wiel fleinere und

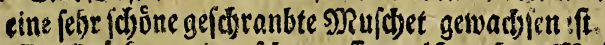

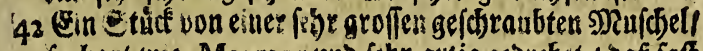

fo bart wie Marmor und fobr attig geireffet / ba b faft

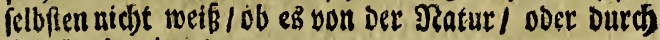
Tunft jubereiset fen.

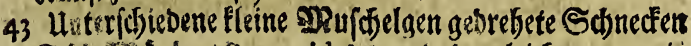

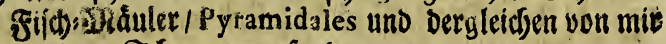
in einem Sdivumm gefunder.

44 Ein monftreufer Rlipp: Slauer von aufen Srofenfars

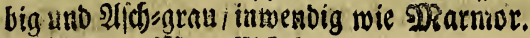

45 Ordinaire s)?err.(Eid)eln.

46 Dren fleine Deftern zufammen getwadffen/ Darauffeis

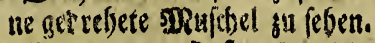

47 Eine andere Deffer Doublette Fleiner / auff welde

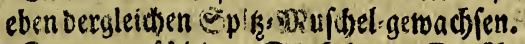

48 Drey unterió(iedene Stadjeln von See? lepfieln.

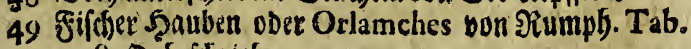
40. \&. D. bejdirteben.

so Ein Moft = Seren / Daran Die Drefungen gleid) benen Sdinecteu jufamen geroadffen/bundele braun von Saro ben / mit braun rotben flecter febr glatt von Escjalen. 51 Eine Edjucter/ meldye mie Nautili oben fich iffinet/

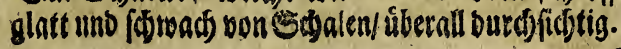




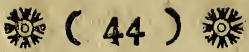 \\ Die andere Edublade Datimnen bie Dubletten.}

Sub Signo B.

(Pe) Ine grofle Pert=-Nutter Doublette.

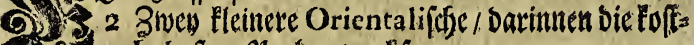
(2) 3 balstfen \$ertrn wadjien.

3. Zmeis red)te Venus doupletten mit Stadtels voer Şaaten / Organon Muliebre reprafentantes, yon Rumpli. Tab 48 n. 4 . bejdsteber.

4 Cine Alt yon 2fufter Doupletten i fo Pinna genam werien/ meís und surchficítig.

5 श2uch cine fleinere gelbidste.

6 Nod) eine anbere sSiot biate.

7 Sime gantş weiffe fleinere als bie vorigen.

8 sin grofles Venus Şeerke Bon. II. 49.

9 Bier fleinerc ctwas neiffere/und inuteribicben an Figur.

Io Eine greffe Drgel-speiffe/ $5: \frac{1}{2}$ zoll lang meif uno gells (idy) beyni Bon. II. 57 . Solenn genannt.

II Eim Shane von orey Soll.

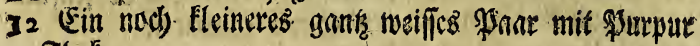
fletell.

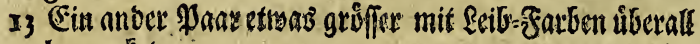
becuertiet.

34 Eine Doublette fonft Sardiniello genafit/ Bon. II. $\$ 8$. Is Drey andereDoubletren fort fdyón/su felfen/Viol blau= er Fanbe/ an weld)er aus bem Centro 4. meiffe Errab)=

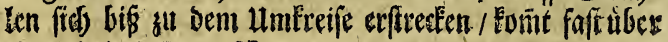
ein mit DeB Bon 11.77.

26. Fine andere yon gleidjer Int / Doc etwas bundflert unb all Dem Limfrei gelblicht.

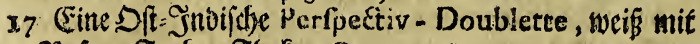

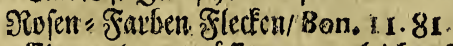

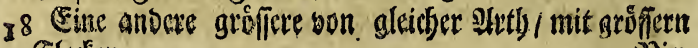
Slectell.

19. Dier 


\section{梏 $(45)$ 耀}

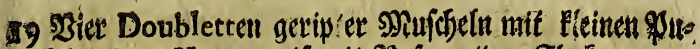

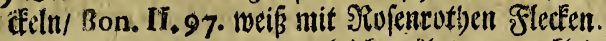

20 Drey Doubletten won gleidfer $24 r t /$ etwas flesiner.

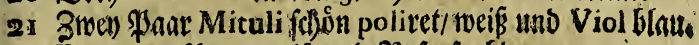

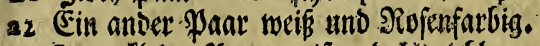

s zwer) Heine \$ant weís und Viol blau.

24 (Ein anber \$aar! glatt von Sibalen/ inwendig Viol blau / augivenbig yon Strof) = Sarben bundelbraun/ Putputr/grinlidbt/ und andern Coleuren / curieux gemialet.

25 . Eine fin etroas blat.

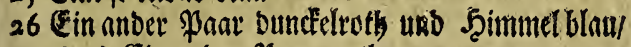

27 (a) Eiu ander Paar rotbe.

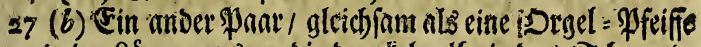
in Ber Ránge. ausiswenoig Duntiel gelbe in Das Sdjwarķ fallent.

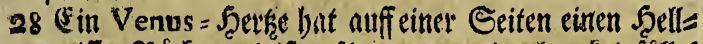

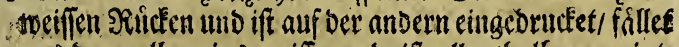
nus Din gelben ins weiffe/ uns ift allentbalben geript/ (el)r curieax.

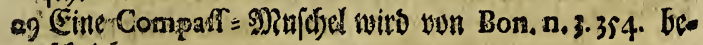
fofricbent.

30 Ein anber:Par felye fboin/ aber weniger sufemmen fílieffeno / als pie burige.

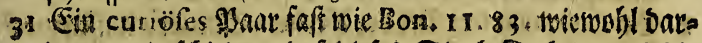

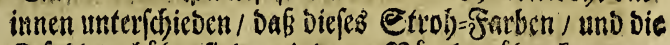
Dácilein buber fint/ uno Denen Sâgeln náber foumen.

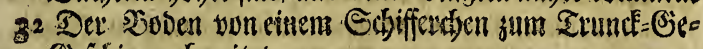
(Sefibiet zuthereitet.

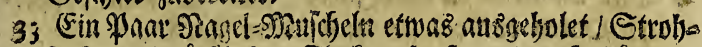

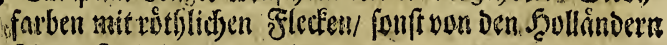
Meterfculpen genannt.

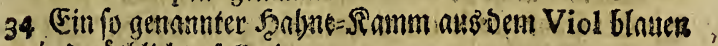
ins vótblich)e falleno

34 Eine Monftröfe Doublette/ attz bem weiffen ins gelbe fallend/binten nuch an ifyem Scjlofie fefe/ uns tam Dajer nichs gans gesfinet werben. 


\section{耀 $(46)$ 䓪}

36. Eine fleine Venus Doublette / wie obien beffortipten.

37 sin glatter Baper auffen weiß und glatt / in wendis nit Chinefiforen figuren fauber gemablet.

38 Gine Venus Doublette forften toop Bestidfte ges naunt / mit Strieffen febr rax.

39 Sin gerippter Gaper/ fiéset einer falben linglideten Defter gleids.

40 Eine fleine Pinne forne ivei仿 unb binten gelb.

4l Eine meiffe Solenis inwenoig gelblictst auffen mit 2Afofarbenen gelben und weiffen Eetreifen felly curiós,

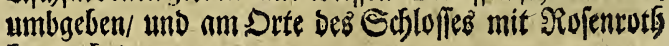
bemerctet.

\section{Dritte Edublabe continuiret Die Doubletten.}

\section{Sub Signo $C$.}

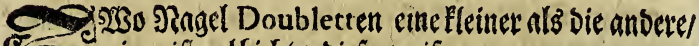
jene iff gelbidjt/ Dieje weip.

2 Roblit=2lufter R.p. I55. n. It.

3 Eine Doublette/ einer Deffer gleids mit bint= ner und surdsfidstiger Sdbale / baß fie nutr wie bas feine \$appier Didf if fâllet aus Dem weiffen ins siof nnesthes umb ift won Eeinem Auctore befd)riebeu.

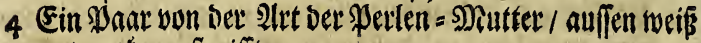
utib gtún geffiteifft.

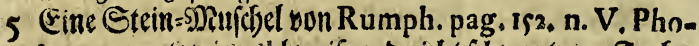

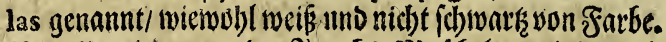

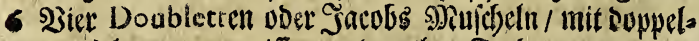
ten Dbren von weiffer uno rother farbe.

7 Dren Paar Spondila an (Siruffe uno Farben unterfobjes Den/ und nicht a woblat aufecinanoer fobliefferd.

8. Siin \$aar grofie lánglid)te 刃suicheln funften Sartari= fobe sjerufdela genanut/ weil fie Dergleidsen braune Soudjtabell abbilben

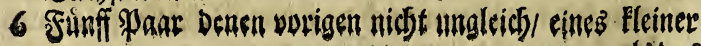
als bas 


\section{紫 $(48)$}

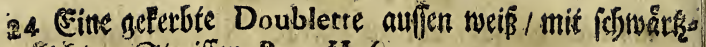
Tidbten Streiffen Bon. 11.6:

2526 Eine andere Etrobfarbene mit Dumfelleraunen

Streiffen/ utio geferbten Deffnumgen Bon. 11.60.

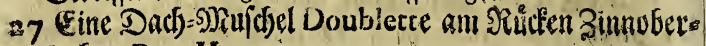
farben Bon. H. 30.

28 Ein 1 elbr weiffer (S5aper/ wie Marmor, mit einem braunen Sabel febr cucieux bemerdet.

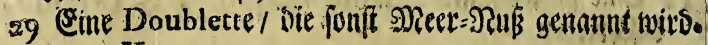
Bon. II. 98.

30 Eine anbere 'bon gleitfer Figur aber glítter/ant Rlicten mit Eircultunten Einien/ uno braun ruthett Punctirten Streiffen / auf meiffem Gitumbe gemablet. 3 I Eine geterbse Doubletce mis filecten uno Sirreiffen bemertfes.

32 Eine geripple Sacobs = Mif fol Doublette van Bon. II. 7 I. bejobrieben.

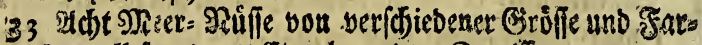
bea/ allefaumt mit Sieculzundosen Etreiffer.

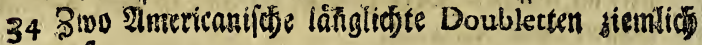
geof

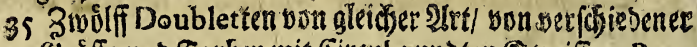
Bjevffe und Farben mit Sircul-sundten Streifer. Bon.

II $35 * 36 * 37 * 40,41.42 .47 \%$ il. Fo m.

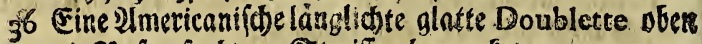
mi $\Re$ ofen farbigen Streiffen bemercter.

37 3nou tånglidite ganks weiffe Doubletten von auffen atlintibaiben glatt.

(38 Idft lärglidfe Doubletten / glatt ann Edjal/ mit sofen - farbigen Eircal sumten Binien auf weifien (3)tunde.

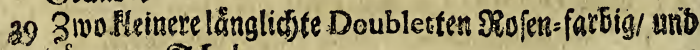
Dixun bon Edisal.

40 3nov nod) Heener etwos runter von gleidjen far áen.'

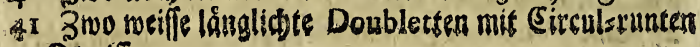
Strrificn. 


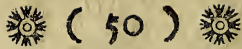

7 Sine anbere former von ङ ben Puncter.

8 গ?od zwo anbere Sfeinfarben mit braunen flectem/ uno febr oundelus siucters.

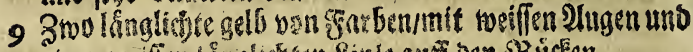
einer meiflen länglicseten \&inle auff ben sRericken.

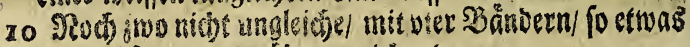
sunctel falles/ quer úbe: gegecosonet.

Ir Eime anoere aus Dem Biol-blauten auff Puepur fals lender 霞arbe.

I2 Eine antere nicot ungleidye mit groffen und fleinen braumen 24 ugen bemerctet.

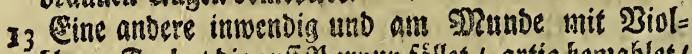
blauer Jasbe / Dis auñ Burpur fallet / artig bemablet/

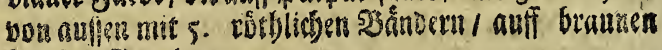
Uteichen Oorunben.

I4. Cine anvere oben meiffe/ uns unten Sraune/ mit febe bundelelgelben Einien und flecten.

Is Ciste andere auff braumer Brunde mit Saffeansfar benen gleften.

26 Cine novere etwas runter yon gleider Farbe/ am 5Runde 3iol blau.

17 Ster lángligte unien braun unb oben Strob= farben mis oreen Erantren Bănoern.

18 Cire anbere unten bundter Eaftanien brauner farbel

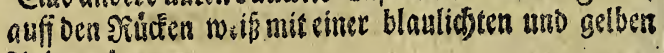
finie umbzogen.

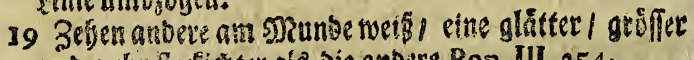
und mebe fiecticeser ais bie andere Bon. Ill. $254^{\circ}$

20 Sier groffe nuD swo flecine unten getiegers / anff Den Slicters sseinfarben mit rutblitben Miablen artig ge-

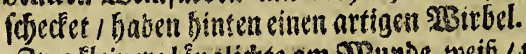

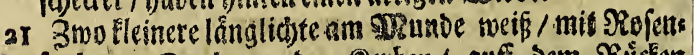
farbenen Epalten ooer Rerben/ auff Dem SRtucten Strob= Farben mit buncteln Rinien.

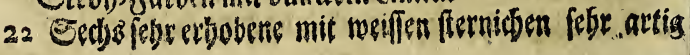




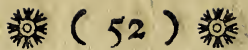

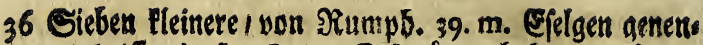

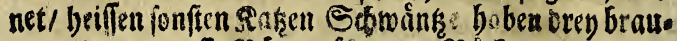

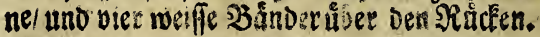

B) Tier andzre mit geterbesem stunde und etfobenetr

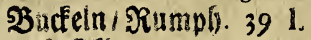

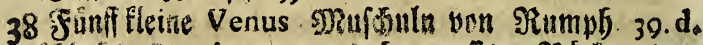

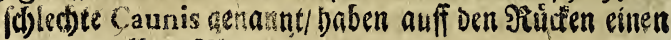
artigen gellen ?ing.

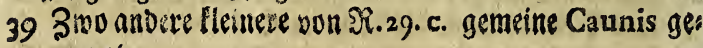
nesteret.

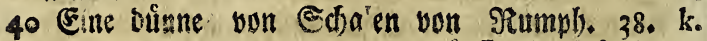
Carneola genannt / wegen Der fininf fflelidi) fartenen כ3änver.

41 3ivo anbere fleine Venus= $=92 u$ (w)eln fonft Cafuarius

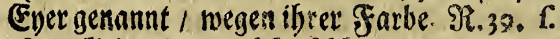

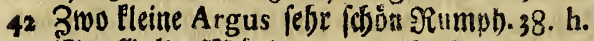

43 Gin Golb = э)?

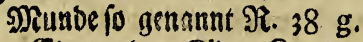

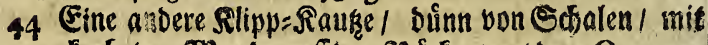

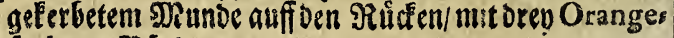
farbenen 2 Bảnoern.

45 Sieben Dracten: Rópfitionen von Эiumpf. 39. z. be=

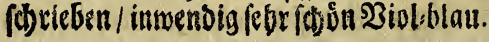

Sumffte Sobublase barimnen Die fogenanten Sirabben entbalten.

Sub Signo E.

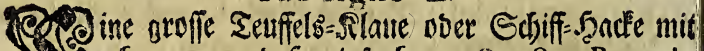
o) 3raunen uno Şunigfarbenten Puneten Rumph, (2) $35 . \mathrm{b}$.

2 Eine See= Strabbe mit Eects Stacteln am

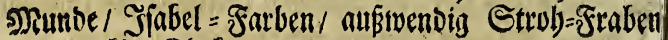
mit bundilen flecten R. 35. e.

3 Ein fo gentunuter Scorpion R 36. k. Bon. III. 3 I2. beifift fonfifen Podagrica, weil tie Epifen getruimmer ferben wie Die Stinger ben Denen Podagricis. 


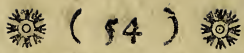

bet Garben / auf ber andern Seiten aber buaktel inb

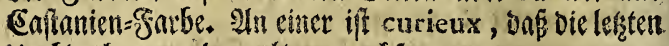
unborebungen bopwelt gewadjen.

20 Ein Casquet mit Zndeten/ uno mit breiten şsellen yon brouner Farbe muff weiffen Getumbe Gemnhlet.

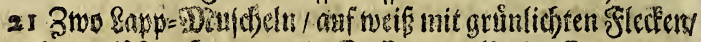
Darstwifhen berum uno Saffen gelbe lanffit.

22 Ein Casquet wie Marmel/ allentbalben uneben / mic

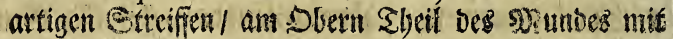

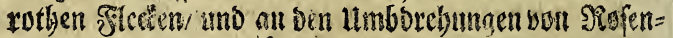

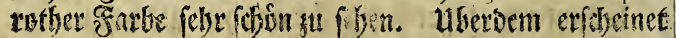

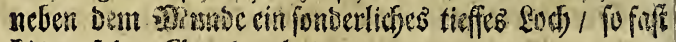
bis auf ben Gintuto geljet.

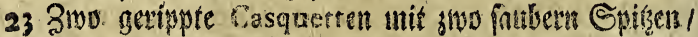

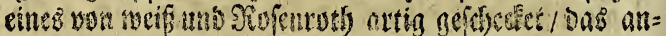
bere mit Enfftan gelfen sinten artig twnbsoge:.

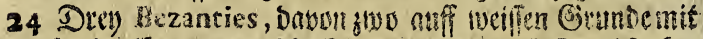

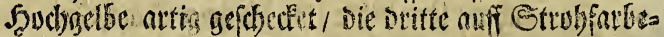
nenen Bitunoe mit SRofentolyen Rinien felse curieux gentablet.

35 (Fine gefdraube Miufdel/ wie cin Casquer getaffet /

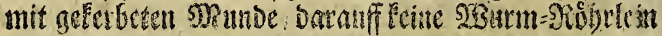

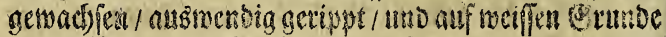
nit gelb bramen filction bemertitet

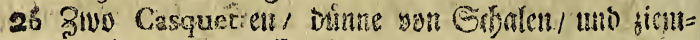

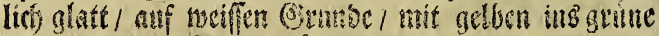
fallenden flectext uno Bimoern gesiout.

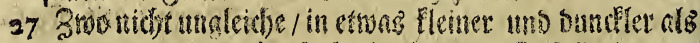
Die anvern/ ats Selfatbe ia has metefe falleno/ mit

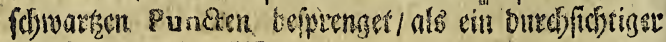
Sandracer, Bon. MII. 49 .

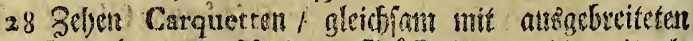

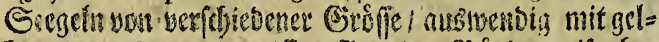

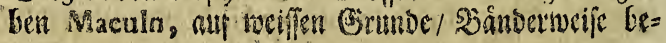
firenct.

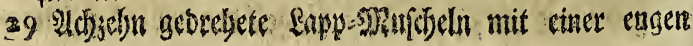




\section{橉 $(55)$ 筑}

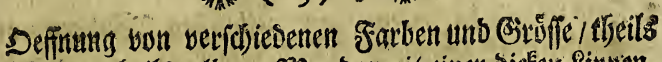

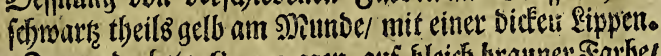

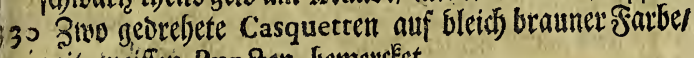
mit weiffer Puncen bemerteret.

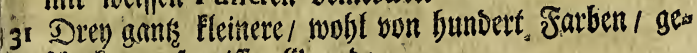
fohertert / auf weiffen (siutube.

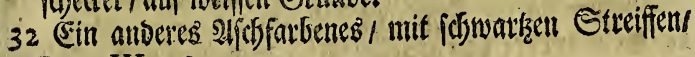
Bon. III. 38.

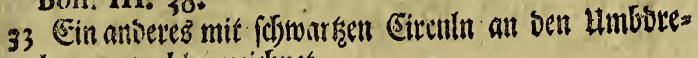
butrgen wobl geseichnet.

\section{Srdifte Sdublabe/sarmen dic Stadel, und Riget Mitudimfor Rumph. XXIV. \& XXVI. beforeibet.}

\section{Sub Signo $F$.}

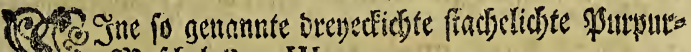

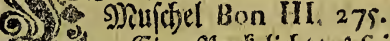

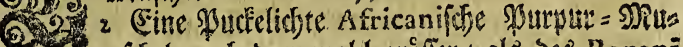
III. 28 r. (focl/ noci) brey maly gruffer / als DeB Bonani

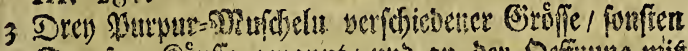

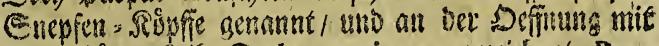

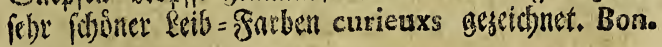
III. 268.

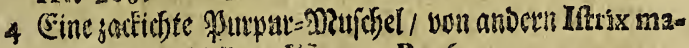
rina gennutht / Bon. 16i. 269. R. 26. g.

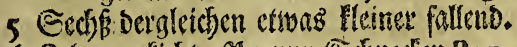

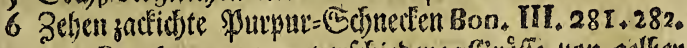

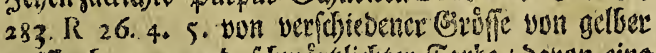

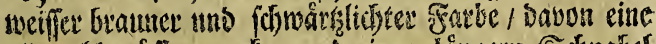
ailemabl grofierere zacten tmo eimen lingern Scjnabel bat als bie andere.

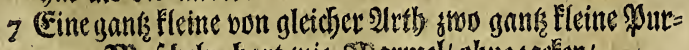

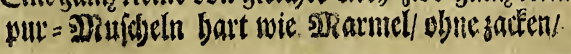

$$
\text { D4. } 83 \text { sivo }
$$




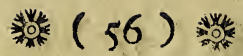

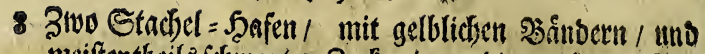

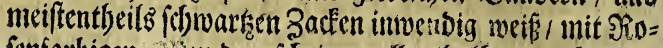

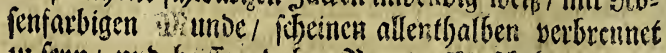

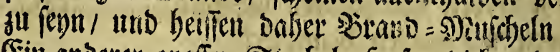

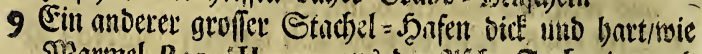

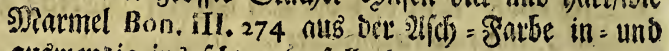
ausivensig ins foswartge fallend.

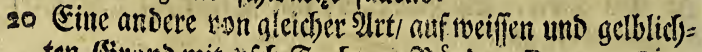

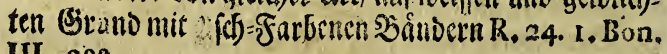
III. 288.

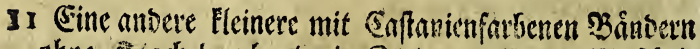

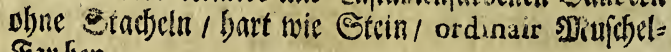
far ben.

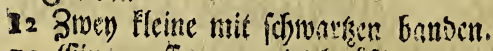

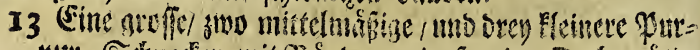

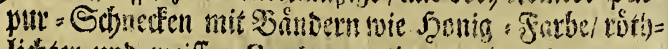
libter uno meiffer Coulcur artibuva ein anter unter= idsieben.

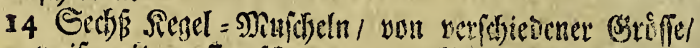

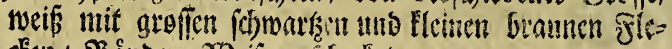

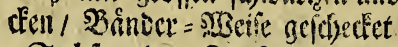

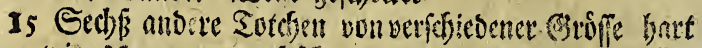
wie Marmor, auf (Wnsursent Estumbe/ mit weiffen fobuppigen flectien/ urtig geciderlet//Rump $2: 1$.

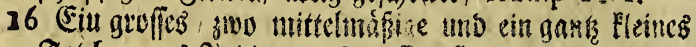

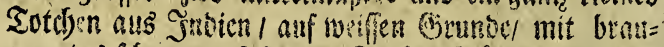

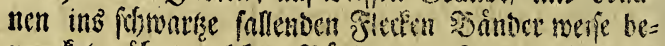

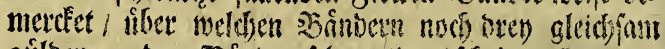
guilbene anbere Sánoer felor artig cifódseinen Rumph. 3I. d.

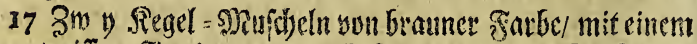

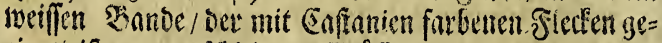

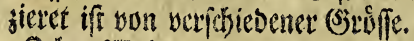

18 3ethen 23 stlen/won Rumph XXXI f. getirgette Sint=

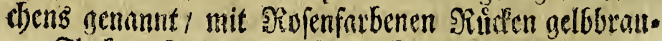
nen Steden / Sinien tveife bemertett/Bon. III. 135 . 


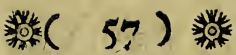

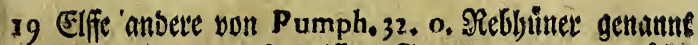
Bon. III 13). nuf weifien Gitunve mitt cu esfen Silutfarbenen Simien und Gollojarbenen Flectin / auf

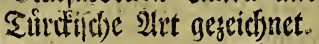

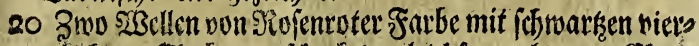

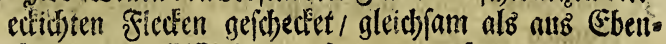
Solls tun Elffenbein bufammen gejerset / Rumph. XXXIII. p p. nernet fie Sauter, Soten.

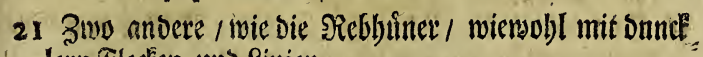
lern Flecten und Sinien.

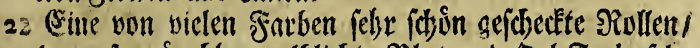

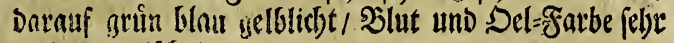
ntrig vermiichet.

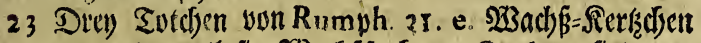
genern t t weel fic 230 ad) farbener Couleur find / am Sinnie fallen fie Violet unt find auf Den Imborelunts

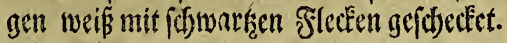

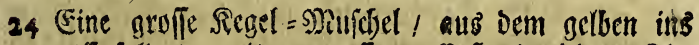

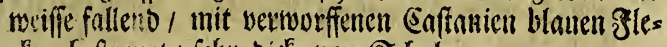

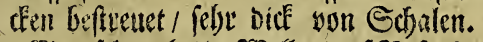

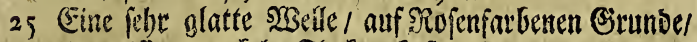

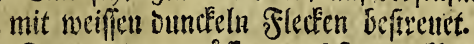

25 3wery andere gróffere/ nebfte : ner fleinern / auf weiffen

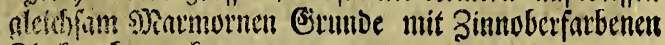
Ffectein bemertifet.

27 (Etite atbere Der yorigen nicfst ungleids/mit brauner

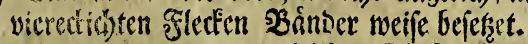

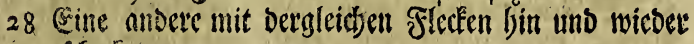
gefdectelet.

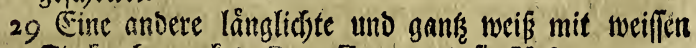
Filecten bemertetet/ $R$. 33. Z, nemet fie Volutam arenatam.

30. Sưnff ansere gants fleine mit weiffen Puncen jiertlids) befintenet.

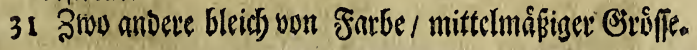




\section{橉 $(58)$ 繁}

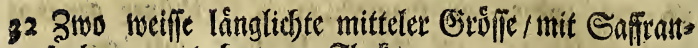
farbenen uno braunen flecter.

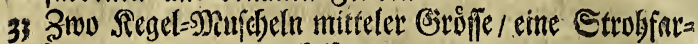
ben / bie anbere sundelbraun.

34 Ulebtgans meiffe mit groffen uns Pleinen Eaffaniert braunen flecter.

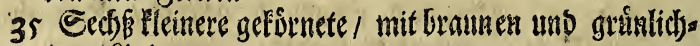
ten Sinien.

36 3wo anbere langlicje mit brounes fleden und vers fóbiebenen fratben febr fóbun gemallet.

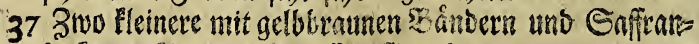
farbenen Einien nuf neiffen Brunbe.

38 Zwen fo genmute Dlir: Belbange/ bon gelbbrauter

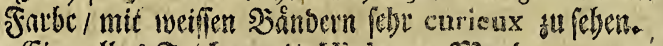

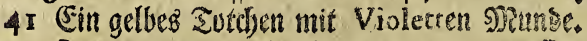

4. Swey anoere wie Sharmor/ mit groffen broumer filediet.

43 3wo Preine Regelmuffoln weis uon Fratie/ Jabou cine Streiffer/ Die anbere Puneten/ bon bríunlibster Fartie bat.

\section{Giebenec Sdoublade/ barmmen meiffens Die Fifómàuler und Del SPuridoln bortemmen.}

\section{Sub Signo G.}

20.j Iebengig Heine weiffe siollen verfblictener Itreen 8) theils mit Buncte theil's nit Pinien/ Sirtifin

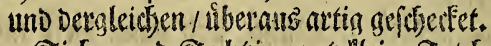

2 Sieben uns Sedbsig gank Hleine Sotcheng/Dar=

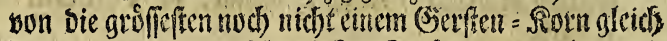
fommen / bon unterfibiestitisen Farben / gemablet / tus

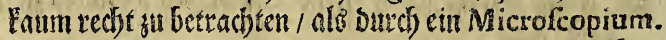

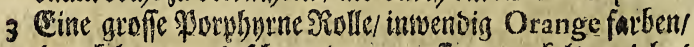

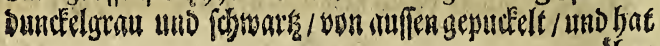




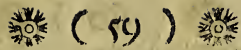

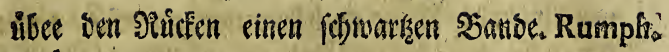
39.1.

4. Drey andere austwendig auf weiffen Erunte / mit @aff tan fartien / getb / brath / utto grinlicfter Couleuz, fojoun bemerffee/ werben fonfen Agatien genennet in wens in Orange = Fanteil R. 39. .II.

5 Siwo antere spordbyene/ inmenbig ats sem tweiffen ing

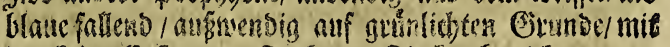
butudefen Enfmatien = Furbenen Fl dien bescidget.

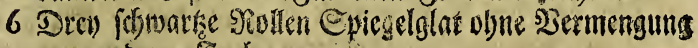
von nascrer Fimber.

7 Gine anbere briunliclyte.

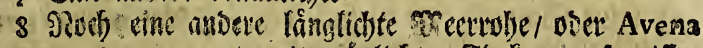
marina genanut / mit grünlicften flecten / auf meiffen Bintunde.

9 3wo andere meiflicbete nit Slofenfarbenen Flectent.

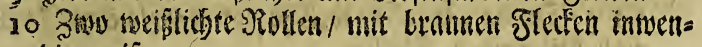
bin weik.

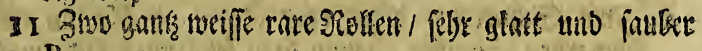
R. 39. g.

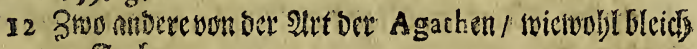
yon क्रribe

13 2lift Sivlen von meiflifiten / grinen braunen/ uno

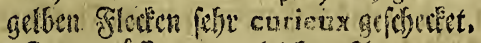

14 Dien groffere won gleiffer sint.

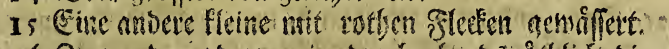

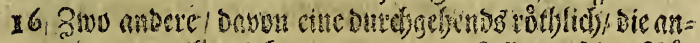

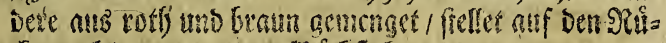

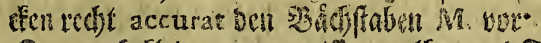

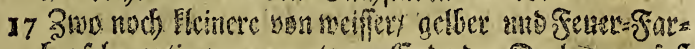

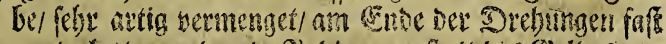
gank platt / metroen in Sndien an fiatt Dis Biclocs ge= brateffert.

18 Bier flesinere ats wcif ins gelbe fallens / nit braumet Punean artig beflecter. No. 23.

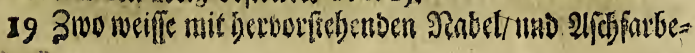




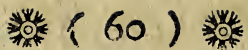

nen Sinien ber Rănge und berunter Gemetrlete / aus unb

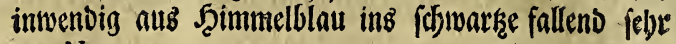
rar No. 22.

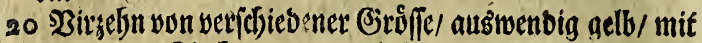

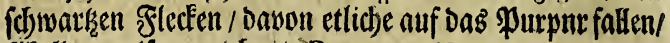
2Bellen-weifie gezeiclsinet. R. 39. 5. No. 20.

21. Drey Regel = \$Nificheln wegen ibser Figur bernt

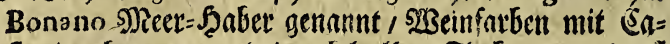

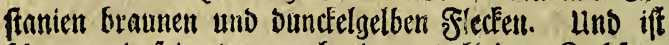

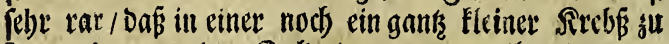
fe'en / Den man ven Evilonten nennet/ weil ere einten ans = Dern aus jeinem Şaulf bertriebern. Bon. III. 57. No. 27 .

23 Eine Sdinnedle/ an Gieffalt betien Fildománlern / mit Statffarbenen Flecten und giferbeten s)? imm / als nit silut beffcét't.

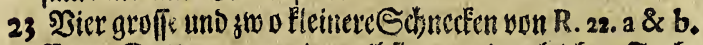
Ener = Dutter genanut / weil fie von bersleichen Fanbe fino mit weifitin gletéten.

24. 3mo Edjnecten/ binn vom Etjal / getborann mit

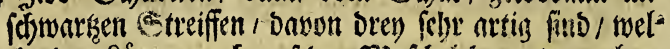

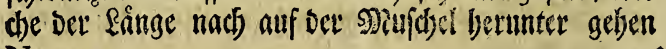
No. 24.

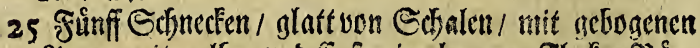

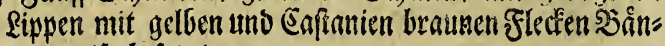
Der iveife bejeretet.

26 Drey anbere mit weitem Munse ganfir weiß / uns an Ben Sippen/ mit biáunlidfter felyr Dunctler Sarbe geseicidynet.

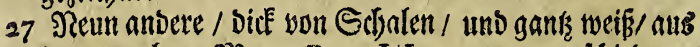
Dim rothen sieer Bon. III. 7. won veribjebener Brioffe.

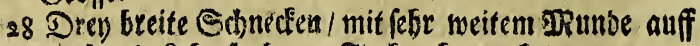
weiß mit Referfarbenen Glecten bemertét.

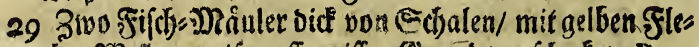

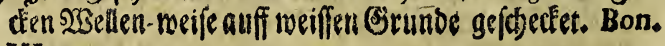
III. 170 . 


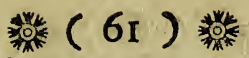

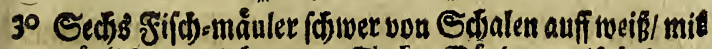

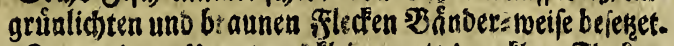
31 3two nubere dito etwas lleiner mit bunctlers: Glecten. 32 (Ein groffes und awo Eleinere/ bergleiden auff weiffer

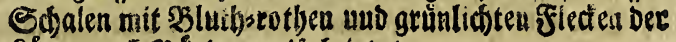

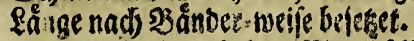

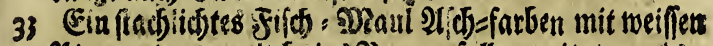

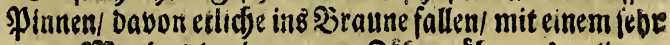

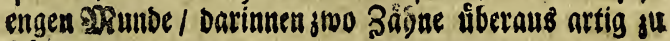
reben.

343 wo groffe und eine fleinere dito van Rumphio val. vate granulate genanat/ auff weiffen Girunbe mit

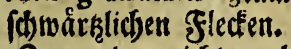

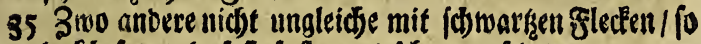

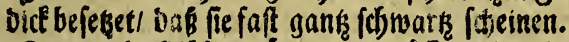

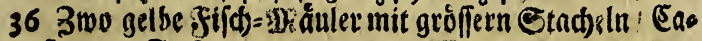
ftaniens Sarben/ mit engea \#Ruave uno artigen ב̊̊̆innen.

37. 3wo Eleinere auff wei init braun und Eaftanien-şats be artig geidertet.

38 Sieben flemere auff weiffen Sd)alen/ mit 2I[d, farbes nen Sinien uno şlecten curvís gegesidnet. Bon. III, 198. 200. 204. 20\%.

39 3wo Pleinere roth) \$urpur: Gatben/ grïn uno gelb ars tig gemablet / eine etrwas butuctiler als :ie anvere.

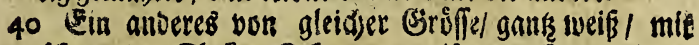

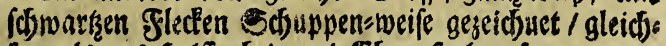

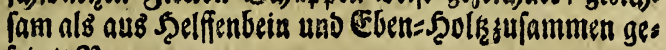

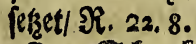

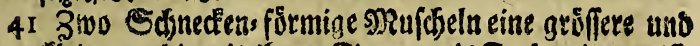
fleinere, bie mit ibrer frgur undifarbe eine meiffe

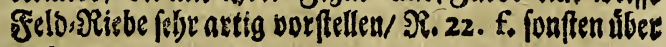
aus rar.

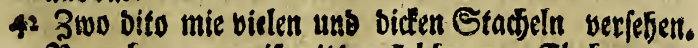
Rumpf. 22, o. weis mit bundel loraunen flecten.

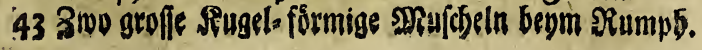




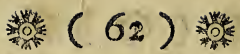

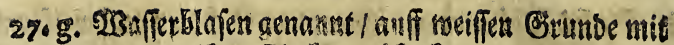
braunen uno gellen flecten sédbectet.

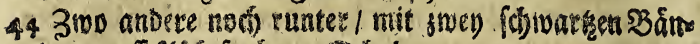
Dern auff $2(d)$ farbener 3 (6) alen.

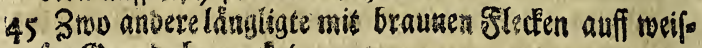
fer: Ertube bemertiet.

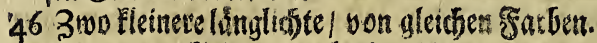

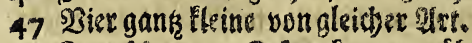

48 Zwo fehr rare Sdinecten von iberaus bitumer uno

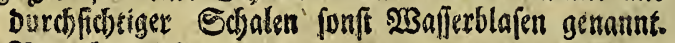
Siumph. 22, b.

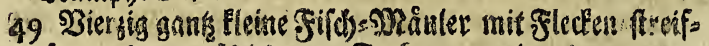
feu uno unteriogiebenen Farben bon einander unter: foriever.

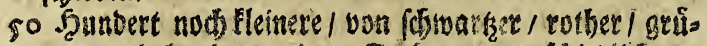

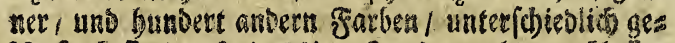

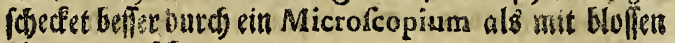
Slugen anjugefen.

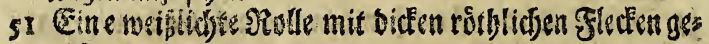
geichnet.

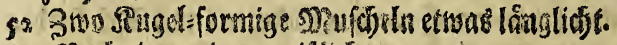

53 9tod eine antere miffliçte.

54 Eine andere Pletnere / auff meiffer Brunde / mit \$urs pirefarbenet gelecter.

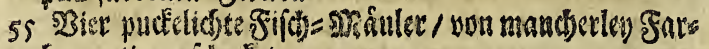
Wese attig gofoctert.

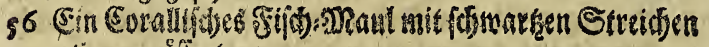
attig gemáfert.

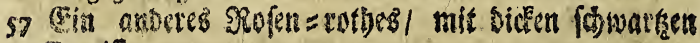
Streifien.

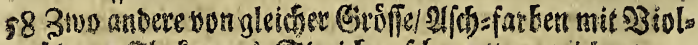

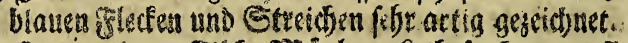

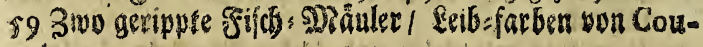
leur.

Go Эier Hleine jowarke Sifommåuler.

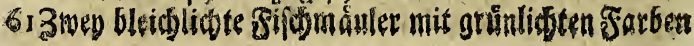




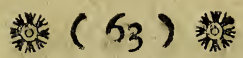

quff ber geriapten @djalen fo artig gemafilet/ baf fie wie \&anb=Sarten anjufeben

62 (Fin anber gुetipptes auff meíf mit \$urpur/gria uno Slut - farbenen flecten artig geseicionet.

63 3wo anbere Straf farbene mit einer braunen Sisto ganç uno gar umbgeben.

64 3ivo noch anbere mit 9iofen-farbigen edjalen.

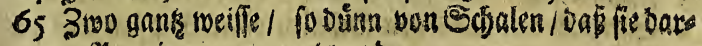
um pappierne getsenuet werven.

66 शeds eine andere gelblidte mit \$urput farbenen Streiffin.

67 Gine anoere gans moiffer mic Marmel/ mit rothticfiet Farbe fhün gewåffer:

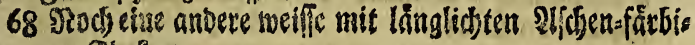
gen flecten.

69 3wey Pleine \$orten farbige mit bielen flecten.

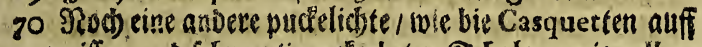
weiffer uno felge artig gefertbeter Echalen / mit gelben uno braunert fltcten.

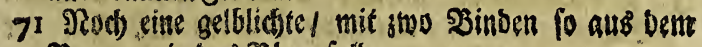
Sraunem in Das SBlaue fallisn.

\section{2dbte Sdublabe / Darmnen bie Casquetten uno groffe ङdnnefen.}

\section{Sub signo $\mathrm{H}$.}

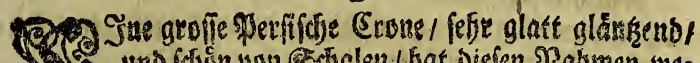

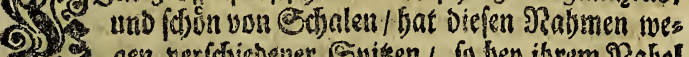

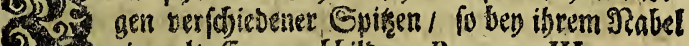
eine alke Erone abbilden. Bonanus III.

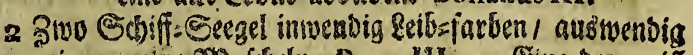

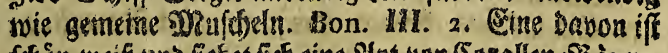

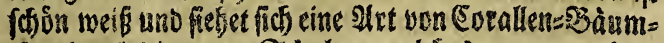

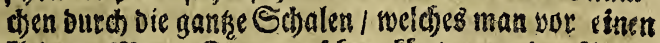

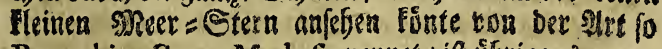

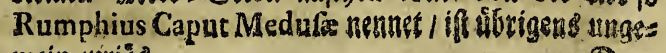
mein curitis.

3 Driv 


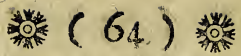

3 Drey Watrifen you Bon. HI. y 9 I. aud Srompefer

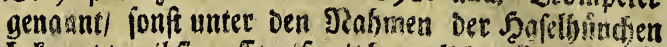

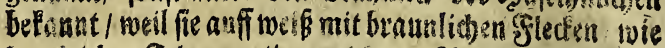
Dergleicher Severn artig gejeidnet. Siumpt $27 . \mathrm{c}$.

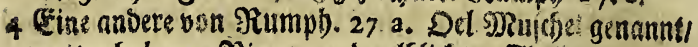

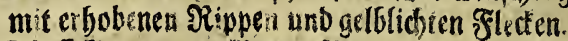

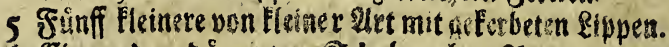

6 Eime andere dinne von Sdunlen ubae 3:ppen uno ges ferbeten $x$ punbe.

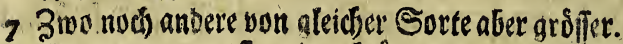

8 Drey ganthe meliffe mit geletimumten Rippen surdsfids=

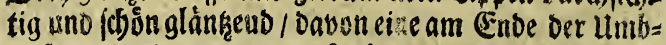
Desefunges eine accurate Sinfe mit redten libenoigen Garben vorfectlet.

9 Eine anbere bart yon Esfalen mit gebuagner sinpe/

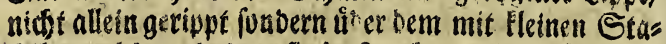

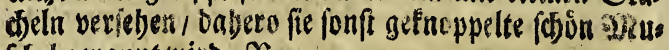
focel genannt twito. $\Re 2$ 27. n. I.

10 Ein Casquete/ melebter. Bonanus III. 16r. Oval= Sdinecte nennet / mit gauts fleinern Etreiffen / cer Eánge nady g: Eerbet.

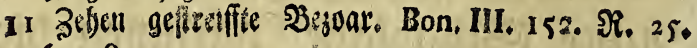
6. 7.8 .

12 3mo andere mit Sraunen uab rótflidsen Gl cleter.

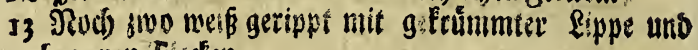
beaunin fricten.

14 Sin meiffes Casquett mit braunen vierectidften fles

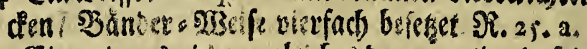

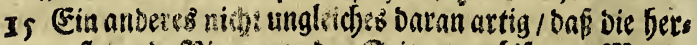

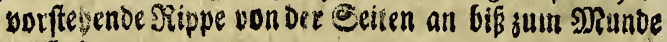
reidhet.

16 Ein anberes Pleines Casquert von Piumpj. 25.b. Fetetben getsannt mit oundéln Fiedere.

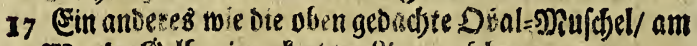

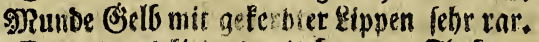

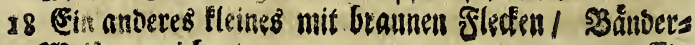
asseife seseridsueto 


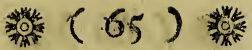

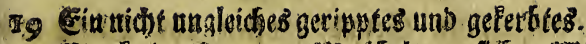

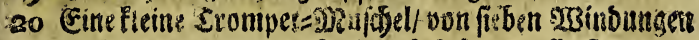

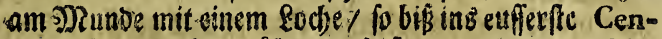
trum binein gehet / fiberaus fejón/mit braunen fles cten gefeidnet / wiro won gimpb. 49, d, mit unter bie zureften gegehlet.

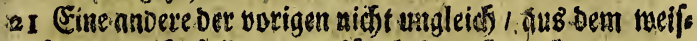
len ins gelbe falleno/ mit lánglioften se treiffen.

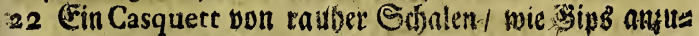

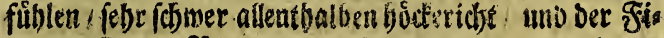

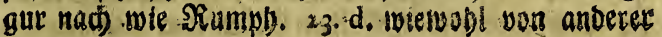
Farbe.

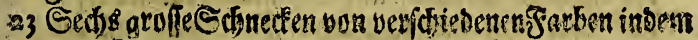

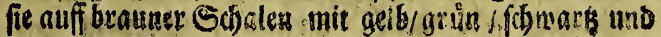
rotb geze dinet / inmenbio Mirlen struterfarbiguno am SRunoe gleidfam mot (ciolde eingejaffet. 3ma von Diefem geigen gle d)iam cine fleitrere Sifnedte / won boulemmener coralen siothel am Ende wrer umb. orebunien.

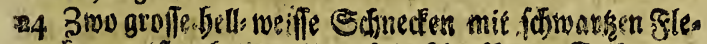

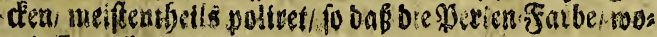

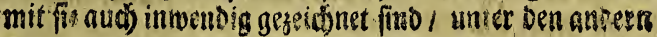
Couleurewartig bers oaipiclet. Bon. MII. I9.

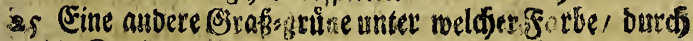

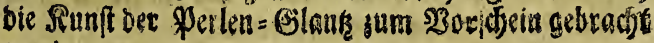
morten.

26. Cime andze von gleidier Grofiffe / auff meiffen Grombe mit fofonen grinen Glecten/-nebft bratuen/gelbes uno

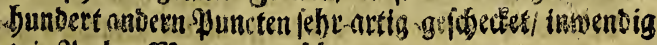
wie \$erlen-Sndutter anzuf fhen.

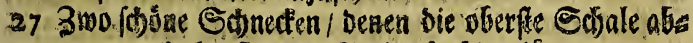
gezugen/Daber fie gank soetlen farbig glianz̧en.

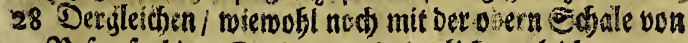
No ofen-farbiger Couleur unb giemlido ungleids.

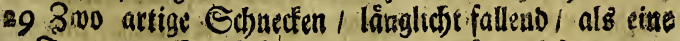

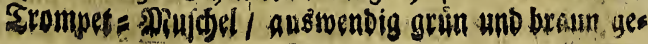




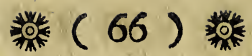

flectet / auff gelben Grunbe fonften obne Silank uno uneben.

30 Eine groffere von gleidjen Sarben mit grófiern SRippea/ und inwenbig \$erlen,farbig.

3I 3wo anbere nit Stadjeln/ auffen von vielen frarben/ vornemlicf) aber rotl) und braun; Inmenbia Pitten glánkig/ uno weil rine febr artig mit Esalb= Sarbe am Shunde bemercfes / wiro fie Daber Esolo=s)Rinbeben ges nainat. SRumpl $2 \mathrm{r}$ d.

32 Eine Saffrait=gelbe Solnedé mit braunen Eircfeln.

33 3no andere fonft Silbers sycuint dsen genannt / aufi

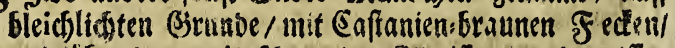
uno über sem mit fobmarkenen Streiffen/ uns weiffen Puncten febr curios versieret / inwendig Perlen: farbig.

34 Eine Edfnedfen aus ber Norb: See oon rôfflid)sfpies lender f̧arbe.

35 Eitue andere lânglidjte auff gelben Brunbe mit vielen eftwas nufünticben Farbent keferget.

363 mo anbere aus Der Siber See gank fojwark / mit ber eufferfien groben seittbe noch iberzogen/ uno Daber in etwas unanfebnlict.

37 3wo Sugel förmige Sojnctêten/ fonft Feizen genannt/ R. 27. k. auff meilen Sirunde mit braunen Slecten/ uni Enftanien: farbenen \$unteen.

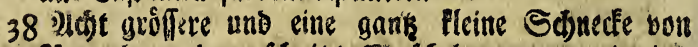

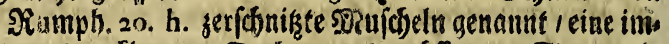

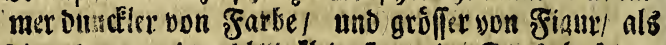
Die anbere/ miemobl sie fleinefte artice Stad)eln bat.

39 Srey andere von Siumpb. 20. I. Spobren genannt। berfen ands ionft Gornen=Sdjnedfen i von benen Stas dela / Die die Strablen der Sonmen vorbilben/ meif unto errbéfäibig.

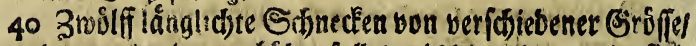
Dav netine immer bojber f llet / als bie anbere/ vier fino aufi weiffen Grunde mit grinalidsten flecten/ swo mit 


\section{紫 $(67)$ 耀}

Yuupurffarbenen/ unb viter mit Erb-farbigen artig bes feket / eire iff braun / und nods eine andere gants und gar wie bie úlurigen inwentig \$erlen= farbia.

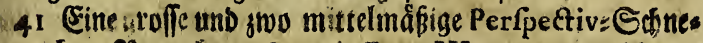
den / Rlumph). 27. 1. unb Bon. III. 25. unter Die ras reften geredinet.

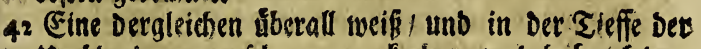
umborehungen fabr rar acterbet und babe id ber.

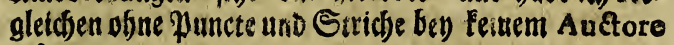
gefunben.

43 Eine anbere mie bie vorigen benber miemobl ofine flecten/ an ben perfpectivifosen Theile.

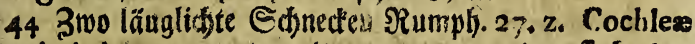
imbrium genannt/ mit gat engen / und auff beyben Seit:n mit geferbeten פRunoe.

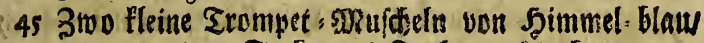
roth uno andern flection uns rarben aefdecfet.

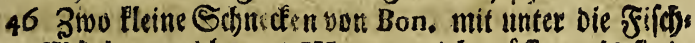
Shäuler gejeblet und III. 322. viel griffer / als fir in

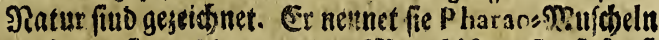

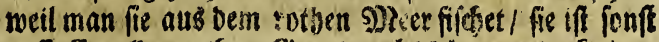
auff Corallen yotben Gizubbe sleidjam mit fieinen fajónen \$e:len uns fojwarken eines umb bas anbrere

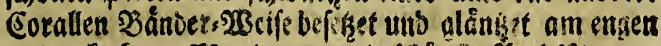

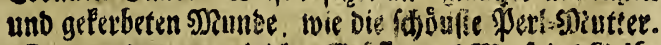

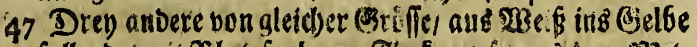
fallende/ mit Blut farbenen Stecten / fo aus tem s)its tel= Pince bis an ben euffertiten umbereis geben artig gefdectert.

48 Sedjs gelwanbene Sduedien / fo audi) glu vieleut unter

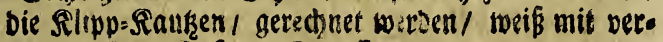
tebrten (Bold: farbener Serreiffen.

49 Sechs Dergleicjen an finte ber Stricfe mit Puncten/ uno gulbenen glecten biferst.

so Eine audere Fleinere mut febr bidfer Sippe auff bleidjo lichten siunde mit weiffen flectin und gilben Strerfifen. E 2

st Eine 


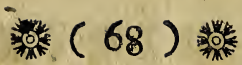

si Gine platte Scjnecle / mit groflem Naube/ Ponft Sdidirgen genannt. $\Re, 27,0$.

52 (Eine Dergleidjen mit braunen flecten.

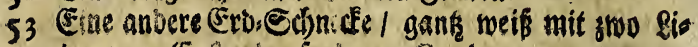
niea / von Eaffanien-farbener Couleur.

54 Eime andere meift runte / Dúnn yon Edialen / und bee grigur nadj faft sie ein fo genäniter Scjiffer.

55 Finff Sáneden/ inmendig Perlensmintter-farbig von auffen geferbet mo gerippt / rotb und gelb-braun artig geicjectet.

\$6 3wo Schneden / Davon eine långlicjt fallet wie bie

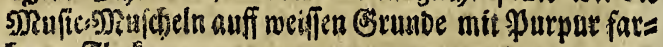
benen flecter.

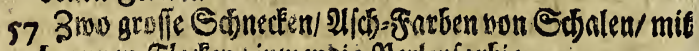
braunen flecten / inmendig Perlenfarbig.

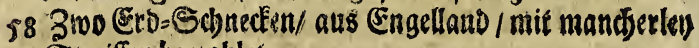
Streiffen bemablet.

\$9 31wo braunlid)te Sdjuctea/ mit Wurpurfarbenen 3̊ănbern.

60 3wo andere rótllidjte / mit Sidjenrotf) beflectet.

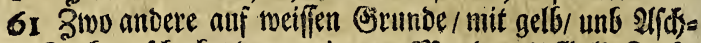

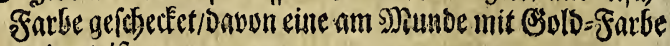
gesietet ift.

G2 3mo mberc Eleinere/ in und aurmendig wie \$erl= S) Sutter glántzento.

63. 3two anbere / Dabon eine ftadjelictet / uno bie anbere ges ferbet iff slodfarlien won Edjalen.

64. 3wo fleinere fefje fladf won Figur, auf meiffen

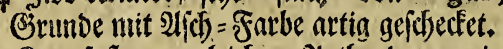

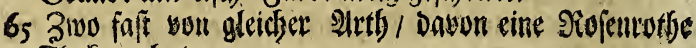
Flettigen bat.

\section{Teundte Gdublade / Darinnen bie Spirmuldeln befdrieben werden.}

\section{Sub Signo $I_{*}$}




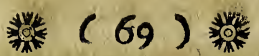

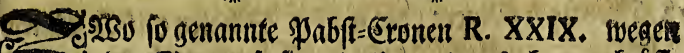
बन. Der Figur/ fo fie mit ibrem irenfacten geftivifi:

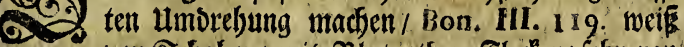
tind theurer.

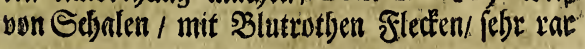

2 3mo fleinere nit 3innoberfarbenen Slecteen / nicht gat zut woblt confervitet

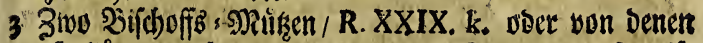

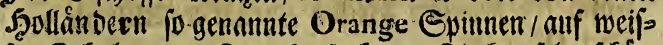
fer Esfalen / mit Zinnoberfarbenen flecten Elebr iss)ith Befóectict.

43 wo Dergleiden etronas fleinerer

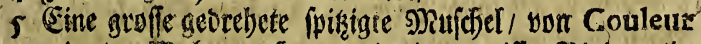
mie eine skobren = Scaut $/$ mit einet weiffen Binbe artig gevictelt. Bon III. 357 .

6 Eine fo genannfe Spille Rumph. 29, f. mit sethen Ltm=

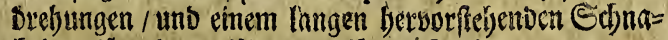

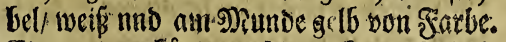

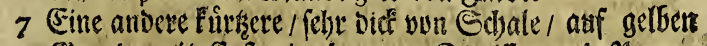
Birtunie nit Eaffenien bratuen Streiffen unb \$imner umgeben. Bon III. 88.

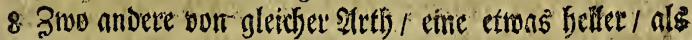
bie andere.

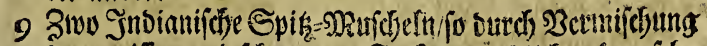

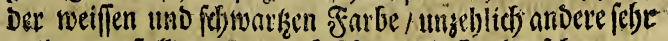

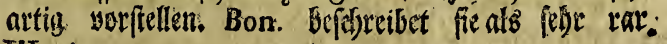
III. 65 .

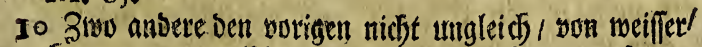
frauner uno getlblidgter Couleur, Siandoer weife ges foreerlet.

II 3lwo fo senannte rauche Srommete

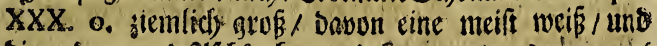
Sie andere aus̉ 2ifchfarben und braun etwas gemenget iff. Bow. III. 69 .

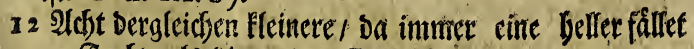
an Sarbec/ als Die anoere Rumph. $30, n$. 


\section{耀 $(70)$ 粼}

13. Drey nodb tleinete / auf weiffen Esfalen mit Caftanièn braunen Puncten / Dason Die Elcinefte audf Dergleidfen Sierbichen bat / artig gefdbecfect.

14 Zmo unbere mit 2 lich farbenen Soindern / allentbalben gefertet / uns ungle di).

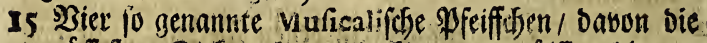
grufieften Siebenzelyen/ bie flemern zmolff mabl umgeorelinetfino/ auf weiffer Sdjale/ mit vier ectidjten Caftanien braunen Sledten gejeid) net. Rumph. 30. b. Bon, III, 1 I 8 .

16 Zwo bergleidjen etwas fleinere/ bnoon eine cin und zwankig umorelyungen zellen fan.

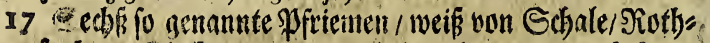

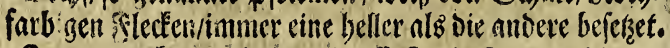
18 Zwo gant Fleine / broon cine Enfranienbraune/ Die an= Dere gelbe flecten bat.

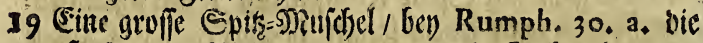

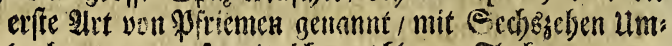

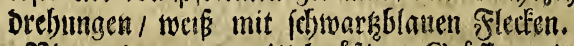

20 Bier anoerc bon mittelmåsigiger Girúfe / mit barten SRippen / 2/(c) = Farbe bon Sdbalen/ eine ift etwas breit= (id) / als eine Erompet= Jierfotel / uno bie antere bat

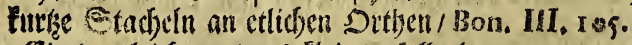

2I (Ein Dergleichen etwas Fleiner fallemb.

22 Nod) fmo fleinere / beybe weiß / cine mit braunen $f(e=$ den / bie anbere mir gelbon Streifen befereset.

23 Drey fo genannte Tubi, wegch ither fiemlidyen \&inge

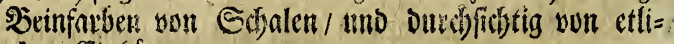
dhen Eingorner genanet.

24 Zno andere twods lingere/ mit adf tschen Unbbrelyungen.

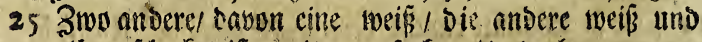
gelb gefthecter iff / mit zuenfadyen unorelsumgen won unten an/ bis oben ju.

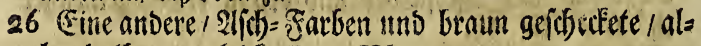
lentbalben unigleidis/ Bon. IIl. 327.

27 3wo andere/von gleidjer Figur und Sarben. 


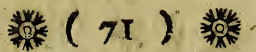

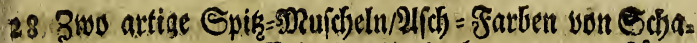
len 7 und an bem Ende Der Umbrelgungen / Die fánge berunter eingeferber.

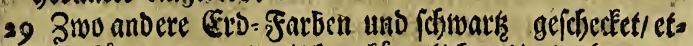

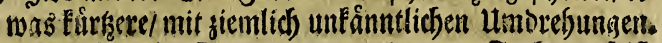

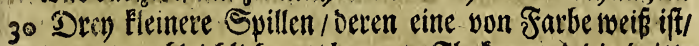

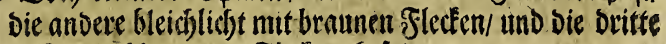

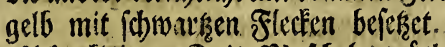

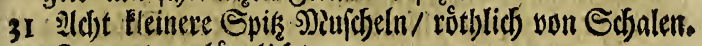
32 3rus andere lángliestere.

33 Eivie $\mathbb{E}$ cinste Enublichte yon curieufen farben.

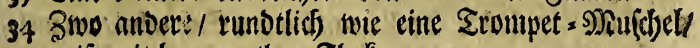
weiß mit braunt then flecten.

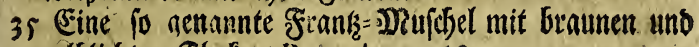
gelblichten fflecten/ Ruinph. 40.M.

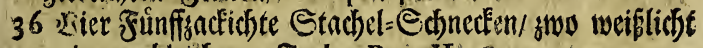

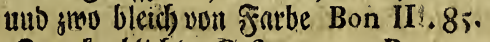

37 Znow Enoblidfte Cafquetten / R. 24. n. 4. brain ge= flectet:

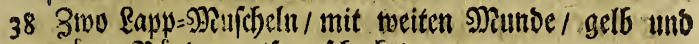

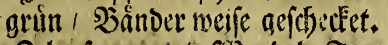

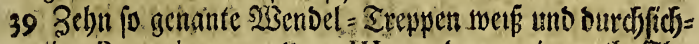
tig/ Rumph. 49. a. Bor. III. 73. Daubn eine vothe f́lle=

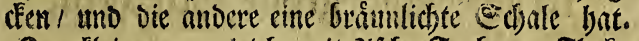

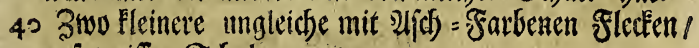
auf weifier Stanlen.

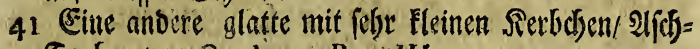
Farben yon Couleur, Bon: III. 40.

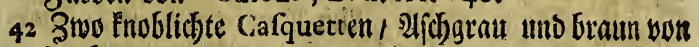
frarben.

43 Eine anbere meiffe metgr linglidgt fallend / als cine इroumpet Muifdel

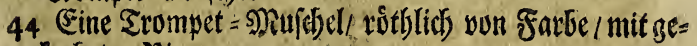
Perfeeten Nipipen.

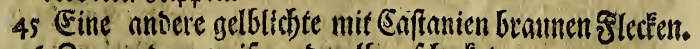
463 3wo andere weif und gell grifibectet.

$$
E_{4}
$$




\section{耀) 72 ( 㩆}

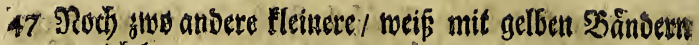
unmidelt.

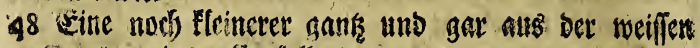
Conie ${ }^{2}$-ing gelbe fallerio.

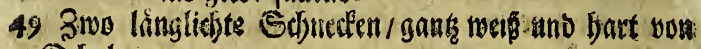
Edyalm:

so Eime ffentere !ónglidtet von R. 30. g. r. Piquenien ge

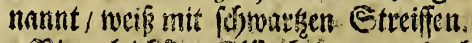

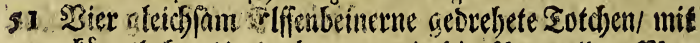

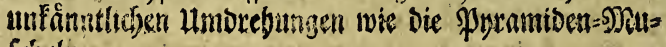
fódin.

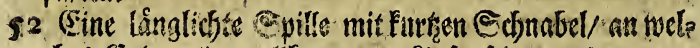

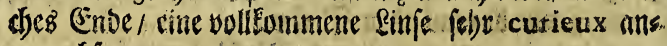
getwadjicn

53 3wo anbere / gants weiffe mit brounen flecten: ichón gro⿰s.

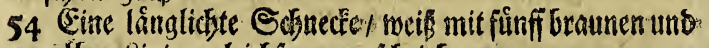
gelben linien gleichjam samichrieben.

ss CEine andere gants gelbe / won gleidjer Grófle und Fio gur, etwas flach geterbet.

\section{Sebenoc Schublabe barinnen Un

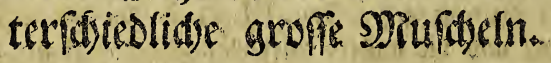

\section{Sub Signo K.}

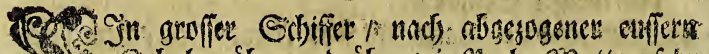

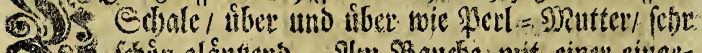

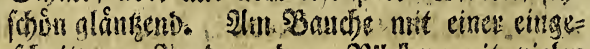

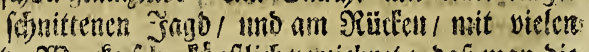

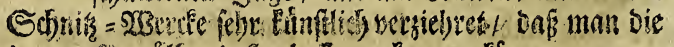

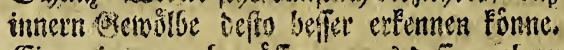

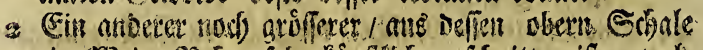

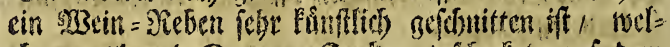

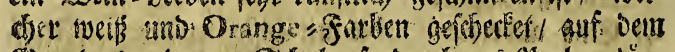

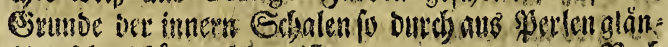

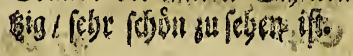

3 (200) 


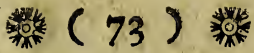

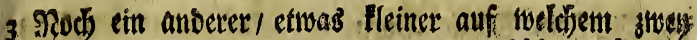

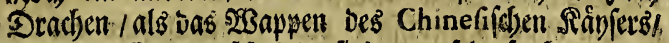
mit einer Erone gefdonitten find / von feble fauberest und Puffibabee: 2lrbert,

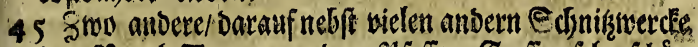

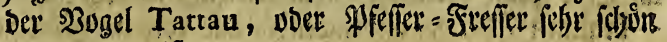
exprimiter ift.

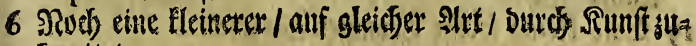
betriter.

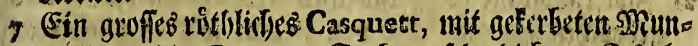

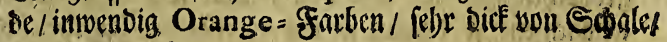
Rumpl. 3 b.

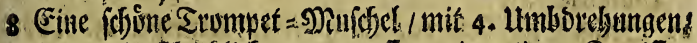
innendis bleichlidfte / won auffen mit artigen Streiffers

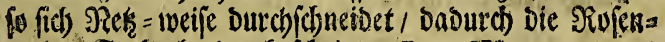
rothe = Farbe herburd) (d)einet: Bon. III. 14 I

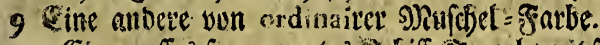

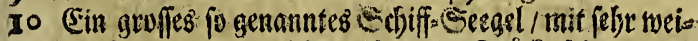

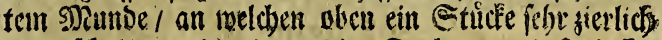

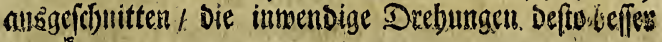
zu erfennen

II Sergleidben / mierwafl etwas Hleiner.

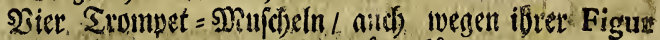

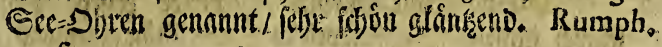
24. f.

II 3two Bezanges mit einer fêt breiten Eippen / nuf Dem

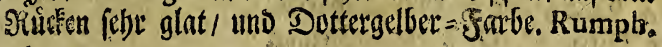
$36 \mathrm{~m}$.

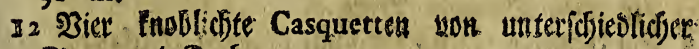
Figur uno fartben.

I3 Eine gleidfiam EIffentreinetme Mufdyet / mit Coffanien braumen flecten fonft Flligel-Şorn genant.

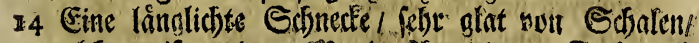

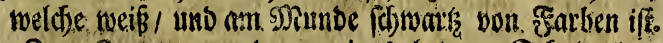
15 3wo Casquerten / Davon eines̉ glat von Sdjaten ong:

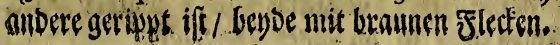

$$
\text { E5 } 18 \text { E.tu }
$$




\section{紫 $(74)$ 耀}

16 Ein flein Muffit Şorn.

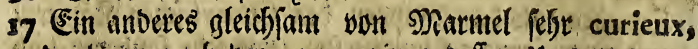
Dertänge nads berunter aeripps beffer umotebungen gegen Dem sane gu tas sie be fallen.

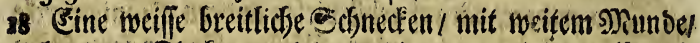
braunen Flecten / unb inmendig aus roth ins gello fal= lenoer Farbe.

19 Eine fleine meiffe Trompet \$naridel.

2I Zuvo Enoblicfte von Dergleichen 2(tet) / Davon eine uffters gebrucfen gewejen/ uno won Satur wiever snjammen

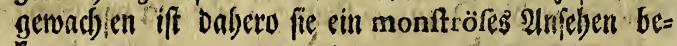
tomaren.

22 Sech) E Elffentreinerne Casquettely/Rumph. 25, c. volt bleici) gelber Farbe.

\section{2ln aroffen grufdeln uno Bdbne= denffind weiter vorbanden.}

Sul signo B.

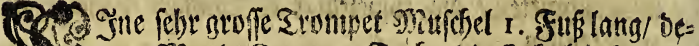

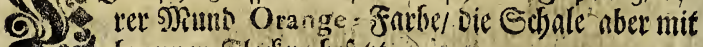
Q.2. Graunen flecten befekt.

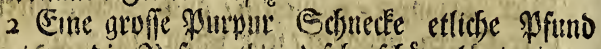

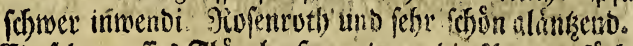

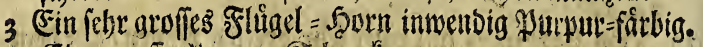

4 Eine ytuffe \$urpur Scinedte.

5 Diro. 6. Dilo ertuas fleinct.

7 Ein ichơn Sromed = Sowen.

8 Ein fleineres glattes dito mit braunen flecten.

9 Dito ctwas fleiner:

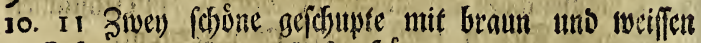
Sduppen gejierte Mulic f̧üner.

I2 Ein Casque von giemlicter Brofle.

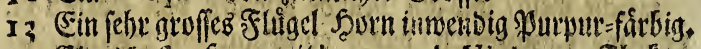

I4 Ein Vuufic Sogen nit braun uno Violeten flectert.

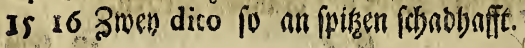




\section{解 $(75)$ ) 耀}

17 Cin brauns Mufic Soun mit Durdjgebenben gatter weifien Eirfeln pelyr fauber.

Hber Dem befinber fich nod) viele bunbert manderiets 2irten Saufdeln / fo nod) auffer Dronung fiub.

\section{Erfter 2(nl)and beftebend ausิ Dielen raren Stúcten Prewbirden Bernfteins.}

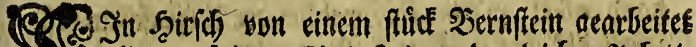
Q) 5 liegt nufeinem Piedeftal von berglciden Antbeite.

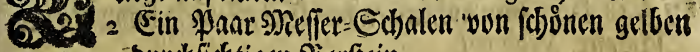
burd)fidftigen כerfein.

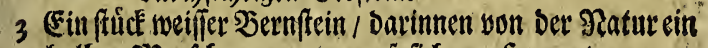
balb r s)ienj(d) won unten nuf fids præfentret.

4 (Ein Dergleidfen Strick/ /D ein \$sappen mit Der J̧elm= Decfe prafentirt.

s (Ein Stief barinmen cin Siupff fid) zeiget/ wie ber Teceli gemablt wiro

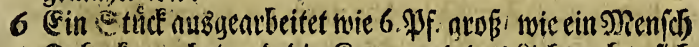

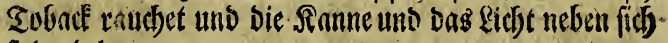
frebend bat

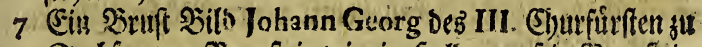
Enchien von Betmftein wie cin STeller grob in SBernftein

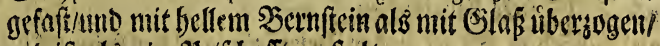

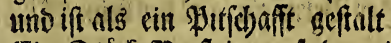

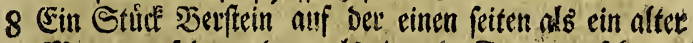
S) anu/ auf ber andern als cine alte Fran anjuffefen.

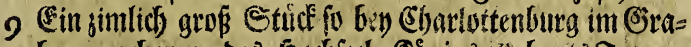

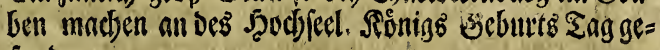
fultben.

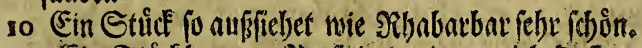

II Ein Ettict brauner jerftein/Darinnen vieles effimungen als unit einem Mieiffel eithgebauten su feben.

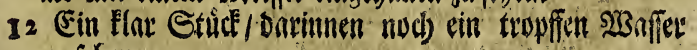
un felen. 


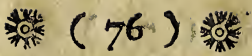

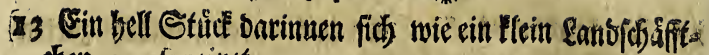
dien prafentirtet.

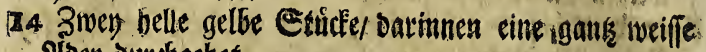
2iber Durderzelbet.

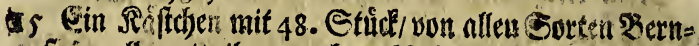

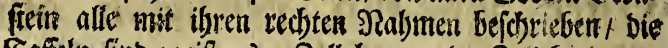
Faffieln find meifitens 1. Zoll lang und 1 soil breit.

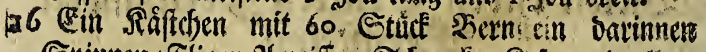

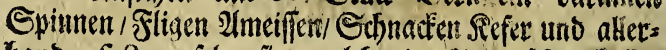

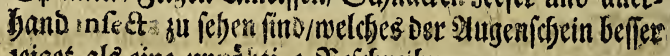

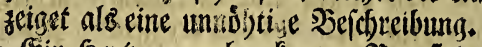

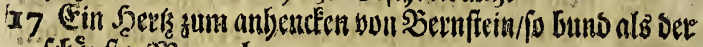

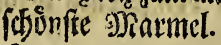

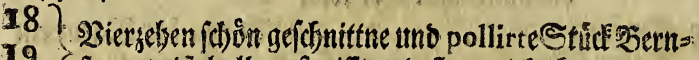
19 (fren meif balb geftreifft uno flammicht bundo/ oak = $20\}$ unter cins mit cinem sidnetenbils.

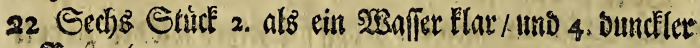
Saphit.

23 Ein stid Dirmftin Darimen cin Fleiner Frofos.

24 Ein Stud Sernffein barim swo Mricten in Coitu begrifen find / eiri curide etiul.

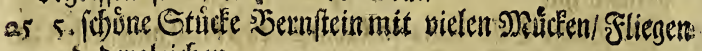

uns Dergleitsen.

26 4: Silute Dergleidsen.

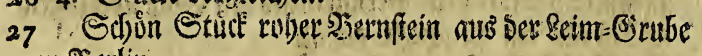
fu Tierlin.

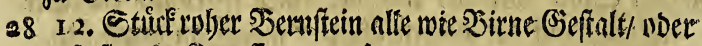
Dá̧ fie als Trovfen gecuefen.

29 Rod) 2 Etide io etwas rumber Dennods sber neeis ftens eine Epitze basen womit fie angefeffen.

30 खsieser 12 sture dito fo aber meif tante fins.

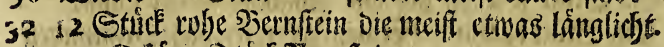
33344 djune Stride \$ernftein.

35 ร. Stuffe unter(d)ievener fratben. 36. 12. Etule diso unferfobiedener Fubber. 


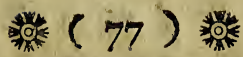

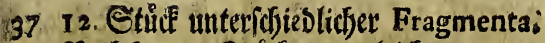

38 Nadb fen 12. Etricten Detgleiden.

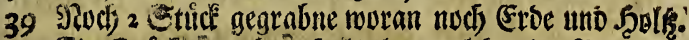

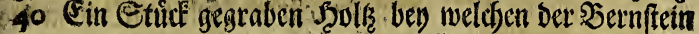

gefimben uno bur bie șkatter beffen gebslten wirb wie

aud) in Sturingen bergleidjen uno Sernffein Dabeng ge

funberi wito.

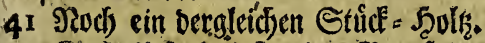

42 Dactyli Judxi ben Dein Sernftein gefunden:

43 Dito io innen bobl

44. Ein fuick bernftein fo in ber Reimfuten bier sejunben.'

\section{Buciter Inbang von Eee (Bewád)}

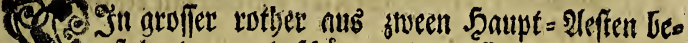
Q) St febender/ und fobin polirter Cotallen Sonum,

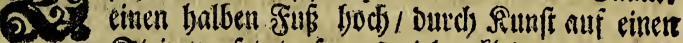
Etein verferzet/ fis aus vielen Hleinern/ in ber See jufanmen gewadjion.

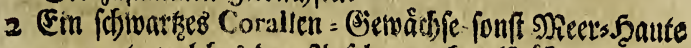

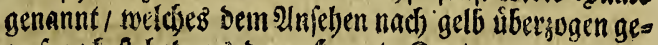

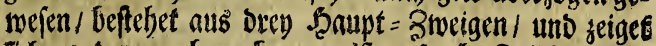

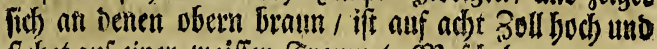

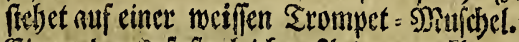

3 Fin anderes feft gleidfer Ant etwas Hleiner auf einer Slipps Staukse.

4 Nod) ein nnberes mit vielen Sinupfen meif betbraun, vben burdbfictsig geld / fitehet ouf einer Voluta.

5 Nod) ein anberes mit Viol. blauer unb geller Materie iiberzogen/won fohr bicken 2lefien/freljet auf Mufculis.

6 פ2ud ein anders beffen einer 2/f mit Dergleichen Mate-

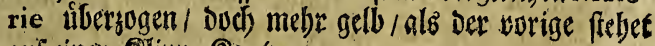
auf einer Siltip = Sautse.

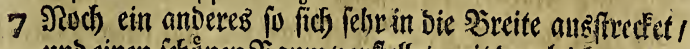
und einen fGiunen Saum worffetlet/ mit Dergleiden Materie meif úberspgen / nuf einet Murice.

8. ग0, 


\section{耀 $(78)$ 耀}

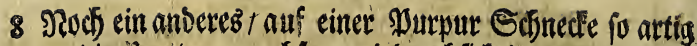

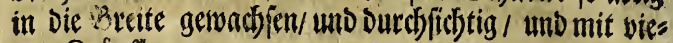
len Rinupfien.

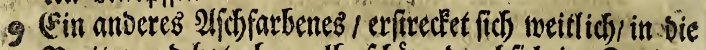

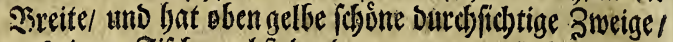
auf eincm Filidbnautl fiel)end.

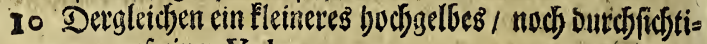
ger / auf einer Voluta.

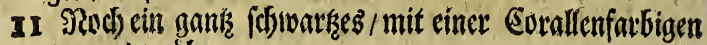
Materie úberzbgen.

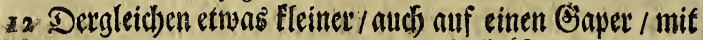
ciner Marerie, wie Florentiner \&ack úber:sugen.

I3 Eine andere fleine burdblichtige/ gelbe Curallen=Staube mit vielen Farben gcjielset.

I4 Cin braun weiffer Etern = Corall / mit eflicfen 2lefien auff Mytulis.

is (Eine weiffe Eorallen = Strube/ woran gugleidf) ein weiß

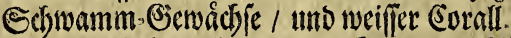

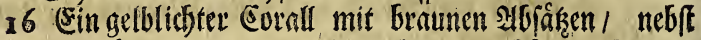
einem braunen/ Daraus cin totber gewadjen/ auf cinem Buecino.

17 Bin tranfer weiffer Stern-Sorall auf einem Gaper. Is Sin weiffer Sornn nebnft einem grauen elern=Eotall 6. auf einem Şaper.

19. Sin Demgleithen auf einem Casquet mit: See: (Eidjeln befleioct.

20 Ein bundfelbrouner Stetn = Eorall / auf einem (S) neet.

21 Ein grater frauler Stern = Corall $/$ auf einem grouten

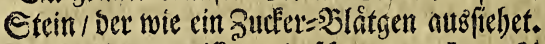

22 Ein anberer weiffer mit fobmartsen Interftiti!s. jouft Hippuras Saxea genannt! auf eimem Ginper.

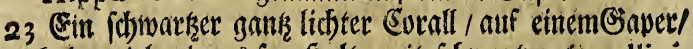

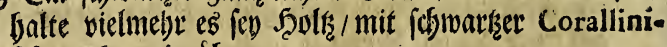
icher Materie úberzogen.

24 (sin amberes Eorallen s Gerwåc) je auf einer Tellina.

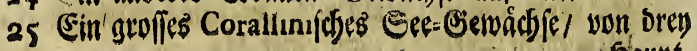
5ुaupto 


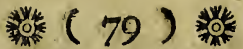

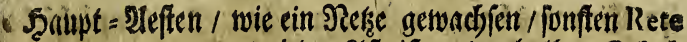
marinum genannt/ jeber 2 ift if anverthalber Edsulb

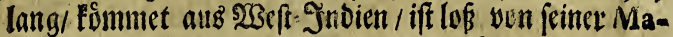
trice, und auf einen Stein fefie gemadfet.

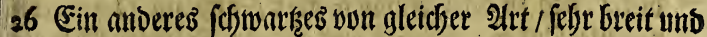

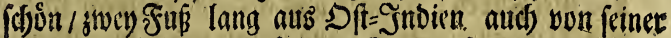

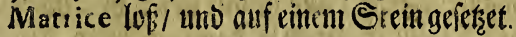

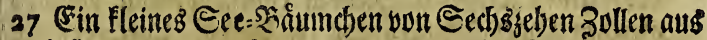
SOft = Snbien / an finer Matrice nod) reffe / an weldher Veftigia, Das nods melbrere rothe Corallen darauf ge= fienden.

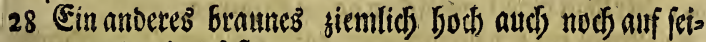
nev Marrice feffe

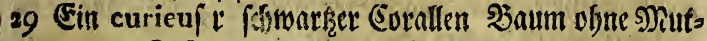

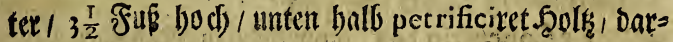
auf perrificiter meiffer Eerrall getwabjien/ aus wels

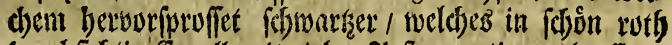

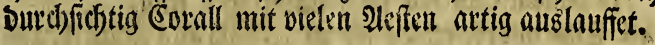

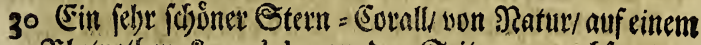

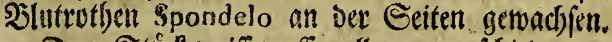

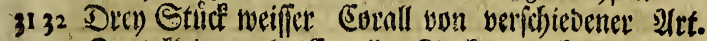
33,34 3wen fleine rothe Curallen-3indien volfifonmen wie

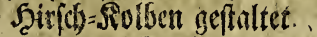

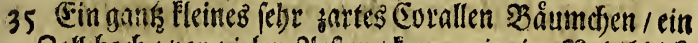

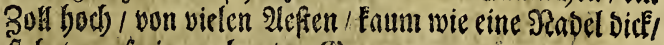
fitelfet/ auf cirem bunten Biaper.

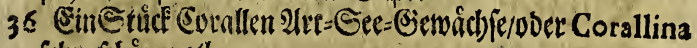
febre ficin rotl).

37 Eir Stick bon einer Erbenen.

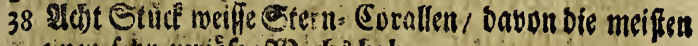

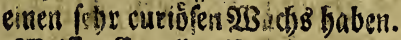

39 2 Beiffer Corallen-SR o⿰

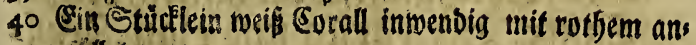
geifitlet.

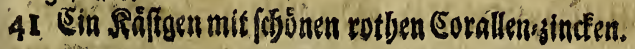




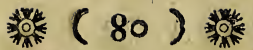

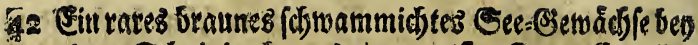

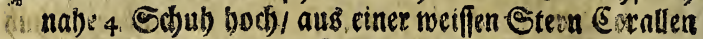

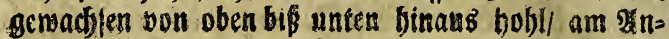

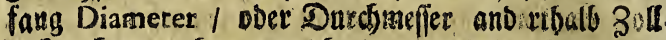
Dideter oben su aber etwas breiter / und im Diametro yon vier 3ollen: Eielet aus als ein ryatteral úber eis

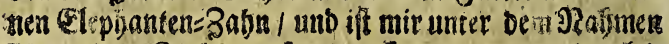
Hercules = Seule fu S.anben Fommen / wor Dergleis gleidten findert fich nirgenos in feinem Cabinet rider

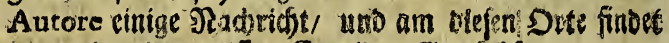

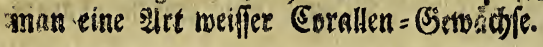

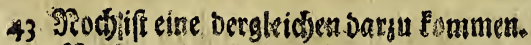
44 Nod ein Dergleichen aber ganz gerade.

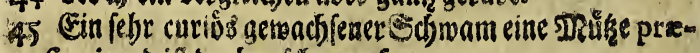
fentixent iff barben fegre geofo.

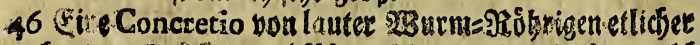

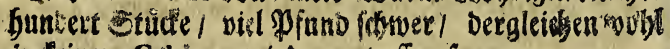
in feinem Cabinet wird anzutreffen jenn. 47 Sine anbere etwas fieinere Muffa aus Lauter Erautern

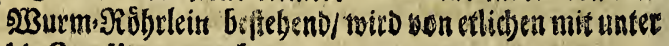
Die Corallina gezedinnet.

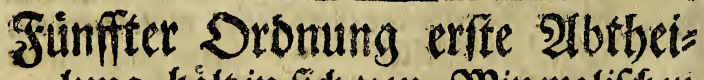
lung, bält in fid von Nineralifosen SReide die Metalla, als (Gold, Silber, Rupfier, SBurg, Sirn, Bimn und Zin: nober,oder Quedffilber= Stuffen, wie aud) Mineralifde Sdiefer und benut Mineralifâte Serg= 2 irten, alg RD=

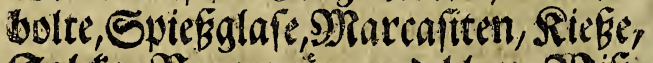
Salefe, $\mathfrak{B e r g}$ grün uno blau, $23 i$ : muth, Spath, Pyrith, sblende 2c. 26. siles 


\section{警 $(82)$}

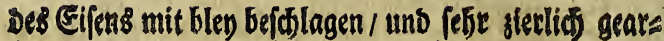
beitet. Sino 80. Stuide gró uno llein.

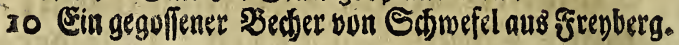

Jerner faffer Der erffe Unterfale in fid Dinerali iche groffe Stücle uno Stuffem/ io nicht in Schubladen liegen fơrnen/ und fino folgende:

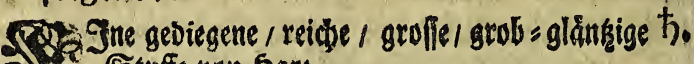
S. Stuffe von Saars.

2 (Eine $\hbar$. Drufe jiemlid fidmet.

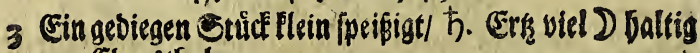
bon Elaustbal.

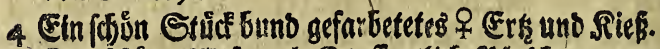

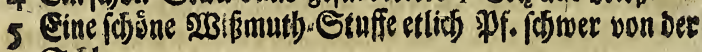
Schleem.

6 (Eine mit vielen Spath vermilchete/ grobglinnagige D) Stuffen ift etwas 2intimonialifob.

7 Gine groffe fosoutre ôfen Etuffe / mit 4 Graupen vers mifojet / aus Eadfen / wiro vor eim fefr rares Stures getbaltent.

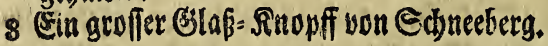

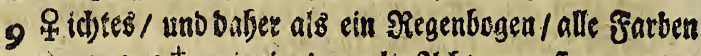

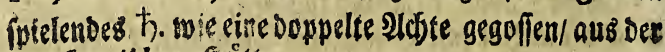
Srenbergilden Soutte.

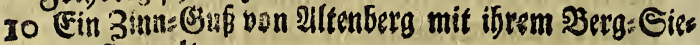
gel geftempelt.

II Duars mit eingefprengten weiß Cidjen uno

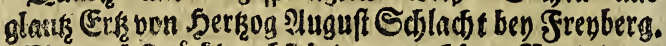

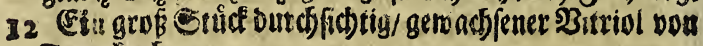
Feandenberg.

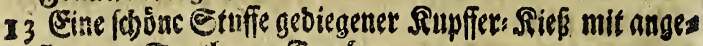
flogenen Spath von Gronberg.

14 Eine groffe Stuffe auggemittert 全 Dites bon Jreys berg.

Is Eino 


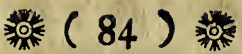

28 Ein grofier vierte balben 3oll Did und einen Guß lang

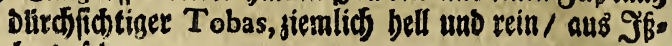
lano febr rar.

29 Eine geoffe Stuffe bou benea 3menbridififen Achac mit vielen bunten Ploern/ und weiffen Eryftalliniforen Quars.

30 Achats Stuffe / etwas Hleinere bock obne Duark.

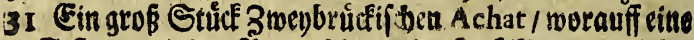
feftung mit ben 2loern febe artig abgebildet bon bielen boc aftimiret.

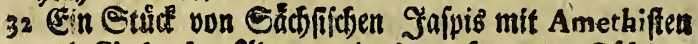
uno Chalcedon 2loern/ wie es Der f̧eer von S/Chirn baufen polirets uno fobleiffen láffet.

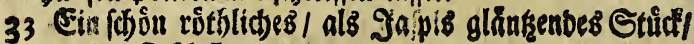
ausier Shlefien.

34. Ein Stuck von einer MRuffienet Diamant: Rugel mobl getwad) fen.

35 Ein ğrofles Stucf Jafpis, Chalcedon uno Amethift ifbereinanber gewasfer/ bun froberg/ wirb nadjoem spoliten fefir fofon.

36 Sutforene Diamant=Stưdengiemlid groß).

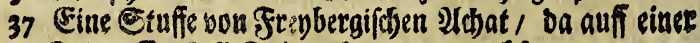
Setten (Sryilalls Spizen beraus getwacb)en.

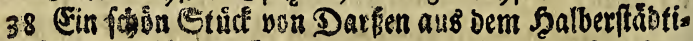
(b)en/ Da felgr viel fleine Peetunculites uno Oitracites nebft Lapide entrocho nno trochite artig Durcbeinans Der getwadjern.

39 Ein Stuiff brauntotber Marmor / in welcjem 3inmos ber re the stoern gefiet.

40 Pietro frumentale Imperati, vid, Epl. Germ. An: 1697. Append.

4 I (Sin gelblicter Stein wie eine io genannte Donners Axt geftaltet/ wiewobl folden Cl Major nicjt vor efo Lapidem Ceraunium will paffiren loffen/ fonbern pro genere armorum berer alten (simbern boult in beren Brábern foldeaucy gefunden worben. 


\section{繁 $(85)$ 橉}

42 Ein Cornu Hammonis, von ungemeiner gróffe/ wels

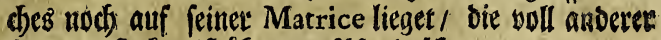

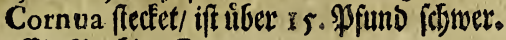

43 Sin Probiert-Stain.

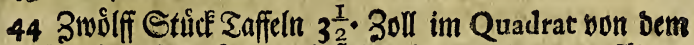

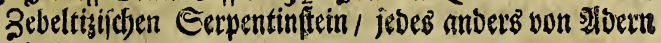
als das andere.

45 Ein fejou Ettide Cornu Hammonis von Altorfi.

46 2ld)tzeben Striuf allerband Marmor Taffeln won als lerbans Brisffe und Farben/mortuter cine io naturtid

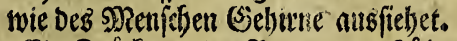

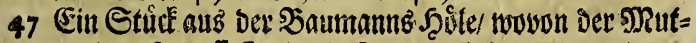

tev/ Dem Iropff:-Stein Offa petrefacta mit unterges metnget ein fotoin greffes Ettide.

48 Ein groffes Stuid' von Dem Tophothermarum Carolinarum, weldyer fids) gat artig ge(d)effet/angebainget.

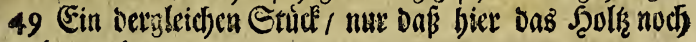
Daran banget.

so Ein Tophus boet petrificirfer Miaop/atu bem Jrun= nen bey Jerra siemlicás grố

It Detgleichen fleinere / nber bâtter / woran man Pein

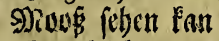

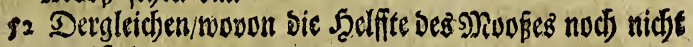
petrificiret.

53 Tophus \& foliis fagi alni \&c. impreflis ex pago Etalten/ im Zuircher Gebirbte.

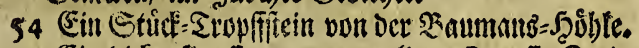

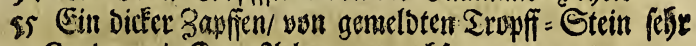

Curios wie Sorn Alebren getwads sen

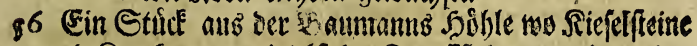

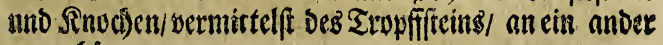
gewadjern.

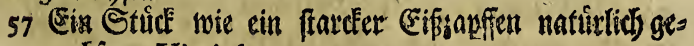
madfiner Vitriol.

58 Ein Cornu Ammonis vonDuterfurt.

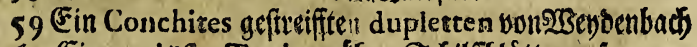
60 Eitt curiöfer Tophus úber Sdjilffblátter julammen rwadjen. 
61 diro Hleinere 2 (nt).

\section{繁标 $(86)$ 新}

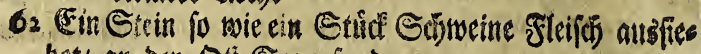
bet/ an ber Dft=Ser gefunden.

63 Ein grofer rotblibfer Etcin Daraus won Cifen ein 5aum getwadjen.

64 Dentrites poer \$aum=Stein yon Sallfelb.

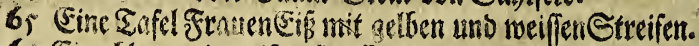
60 Eine blau mo meíp geftereiffe Terra figillata 2 Stude. 67 sine groffe Eliriftallen-T rufe ifgón gewadjen.

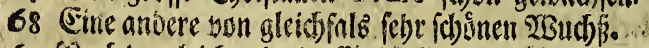

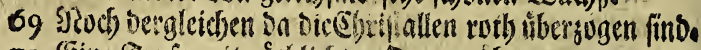

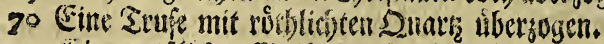

72 cinu weislidote Elyrifgellen $\mathfrak{E}$ tufe.

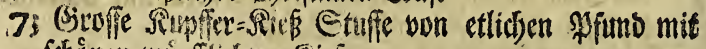
ichomen winfilioten sies.

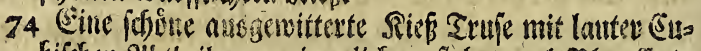

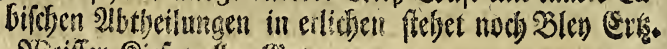

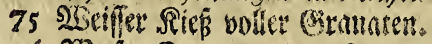

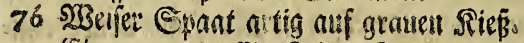

77 (sine curiöfe Ebrifallen=-Stufe van Inneberg.

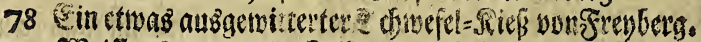

79 2Beiffer paat mit Silber und 2iten=glung uben mit gethen Siens überzugen.

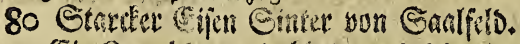

8i (Fin Conchires mit blatten dubletten uon Duerfurt.

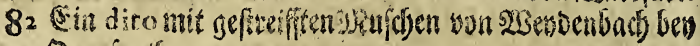
Dureriurtly.

83 dico unit wielen glatten uno Hleinen georelyeten rechuedera

84 dito mit vielen Heinen Siatujeln.

85 Ein Stehin mit vidln grofien uno gants fleinen felbe

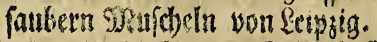

85 dito ba sic bratuc Edjonle fids noch diftinguiret.

87 dito mit lang geovelgeten und vielen anbern.

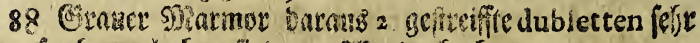
fauber etlyoben find von sisenoenback. 


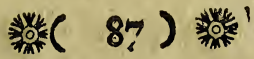

\section{9 dito.}

90 3mey groffie Stúck Sinotien Der cine im Diametro

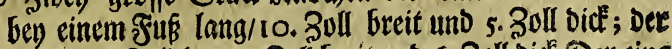
ander 11.30 of lang/9 3oll breit und 6. 3orl bick. Der eine Dabon iff burchaus Sedfwammicfst/ bas anbere aber iff noch etwas mit barten Sinecten íberzogen. Daß́ ę yon

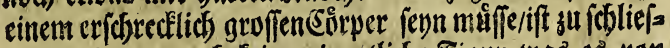

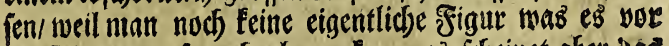

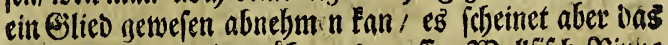

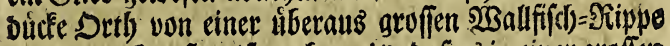
zul fenn. Eonffen iff nodf curiös Daf es in einet groflent Etant alb man ein SErab in einer Sirchen madjen wolo len/ nebft noci vielen andern groffern Stúden gejunders worber.

91 श20d) ein Etice aber Pleiner.

92 (Ein Ervftallen Drufe/ iv inwendis bohl und als eit Casquet gemadbern.

93 Ein 3 echer von Serpentin Stein ein Dunnt baltend. 94 Eine groffe ङutter \$lidjfe bator.

95 Eine jojone $\hbar$ Dorufe mit Eryffallen und angefloges nem 全 Rick.

96 Ein Hlein \$natien Nill son Carlsbab Stein. 97 Ein Róme von eben Der Xyzateric.

98 Eine Rutud yon butnten Marunds, Boll imDiametes。

\section{Der Crfien \&btboitung}

Non ber Finfitien Soromung

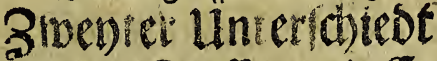

Sált in fid die Stuffen und Sretse von Denen fieben Metallen / als erfflid allerband Bold: Erre aus allen Sheilea Der 23 elt.

$$
\text { I. Gold: Ertse. }
$$

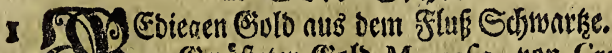

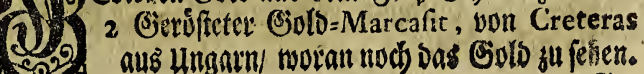
aus ungarn/ moran now) bas Gollo zu fehten. 


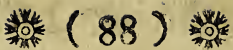

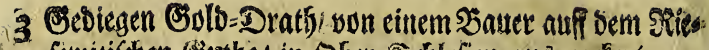

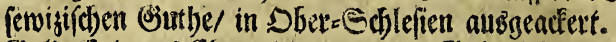

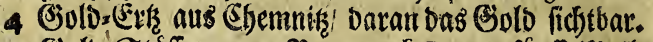

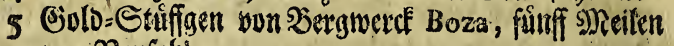
von ఇeuffoly.

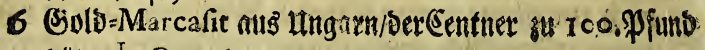

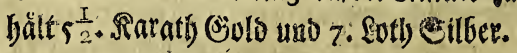

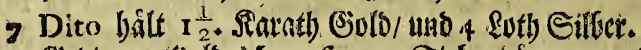

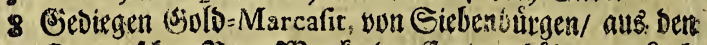

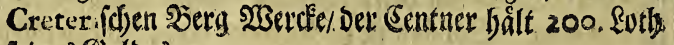
feinis Goloeb.

9 Dito fhin ausigeriftet won felbigem (Sebuirge/ ift abee taub

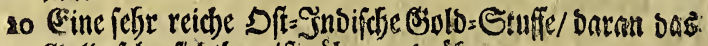
Golo falye fidstbat ift tiber uno siber.

II Dft Intiches Guld=Stuffigen / ba ber Eentner snolff

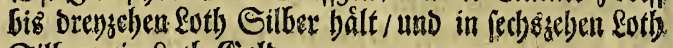
Gilber/ ein \&oth Ģolb.

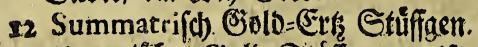

I3 ungarifores Golb Stuffgen weif uno roth gulltig an= sulelien.

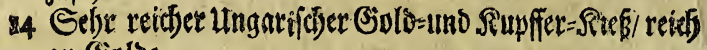
an Sivloe.

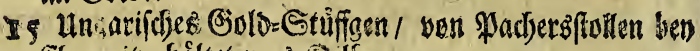
Ehemnits/bâltet tons Eilber.

I6 Eemeine Golb-Eetten / Darnus in Ejenmits bas Solo geronjdyen mito.

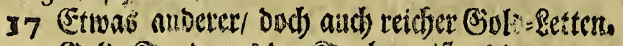

I8 GSslo= S amb aus Der Sanle meiff gediegen.

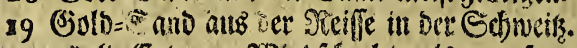

20 $9010=$ (E)

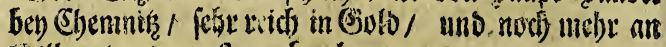
(Silberi in Augufto gebrodien.

21 Eiue Stuffe won ber Jceiligen Dreyfaltigfeit / Berg=

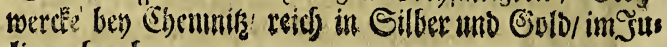
lio gebrodjell. 


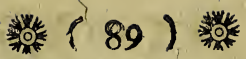

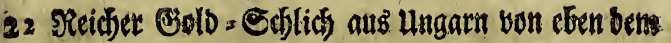
Drtie.

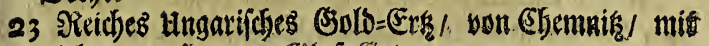
wielen angefiugenen Bilaş-Errge.

24 (S)

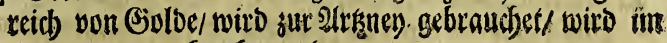
Septembre gebrodjen 1699.

25 (S)

26 Etwas anderes.

27 Giediegen Galo=etuffifgen/ in weifien Duarkge.

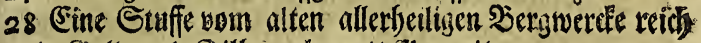
in Giold und Silber/ oljnweit Eljemnirs.

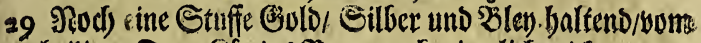
beiligen Drey Risnigs sergmertfe jiemlicf veich und at= tig getwindfen.

30. (Eime Etuffe von \$edberfollen recid) an Eilber und Bulbe.

31 Eine Etuffe von Sanet Michaelisetollen fálbrețileyf Gold und Eifber /ift aber reidfer in Boldo.

32 (Eine anbere von eben Dew Drter weld)e dato Das befte. Eert an Esols und Eilber bált.

33 Ecblids yon foldsem Erake.

34 Guld und Eilber woul \$acterffollen/fo wor rar gebals

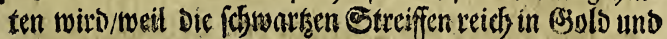
Eilber. Die rothen werden Zinnopel genarnt / auf

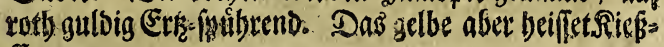
Evis.

35 Eine Stuffe bon glansen গ3ergmert: in Shemnifz/einers

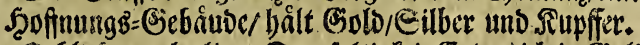

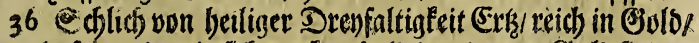
bas wer bamit fidjern fan balb DaB (autere Golb finbet) in septembr. aebrudsen.

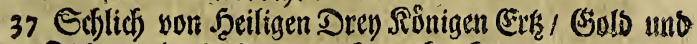
Silber balterio/ im Augufto getbrodsen.

38 Eine Etuffe von einem ganks nar amelegeten berg

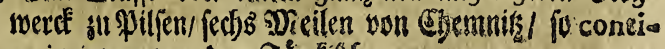
nuiret/ war uor Dem Seitchifich. 


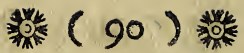

39 Bolb und Silber baltige Stuffe von scilif Eotte

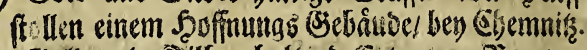

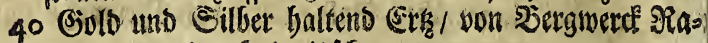
benftein bey ber Scuderifife).

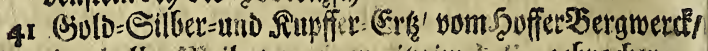

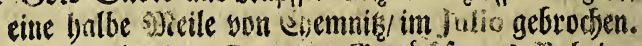

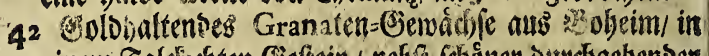

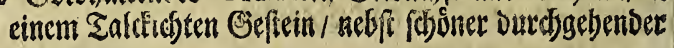
Lithomarga。

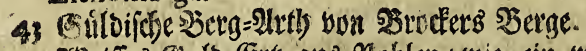

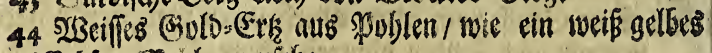

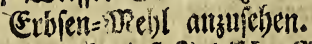

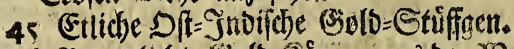

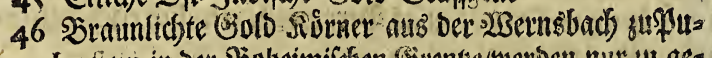

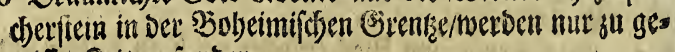
nifier Zeit gefumben.

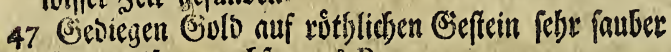
Scanrweife gewadbien aus Peru.

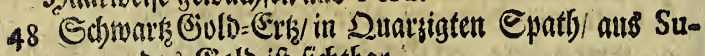
matra, Das Gulb if fictetbar.

49 Bidbfrifficen nus Potoli, mit gebiegen Bodlo.

50. Sndianild) braun Erts mut cingefprengeten gebiegenen Golde.

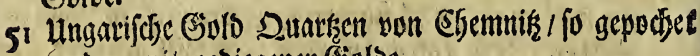
werven mit gediegenen Gioloe.

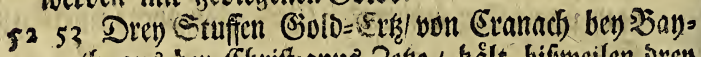

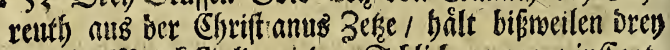
bis vier Minref Esvlo/ wiro zu Sdjlid) gejugen/cinEent=

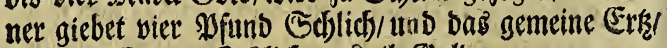
aus bem Eentner Sflid 18. Roth) Ëplb.

\$4 55 Gublofieffe aus Geffowiz in Shatingen baft Det (Eentner Drey Ducaten Biold fino 5. Stufien.

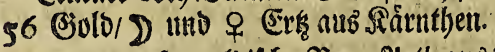

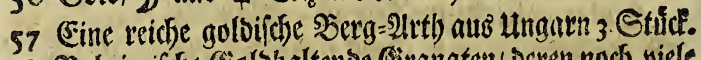

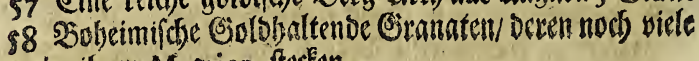
in ilfret Matrice frecten. 


\section{珰 ( $(9 \mathrm{I})$ )}

59 Invere fo reidjer von Golb aus zebliz in Sachento

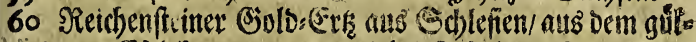

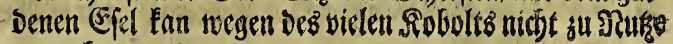
gemndbet werben.

6i Sido. Oslimmer

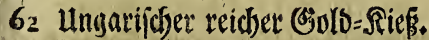

63. Gievingen angeflogen Gold aus \$eru auff weiffen Duaris.

64 Edele (orine weldse felye teids) in Guld.

6; Gold uns D) (Er aus ungarn.

66 ungarifdje Sivlo=Stufe mit reidsem $\odot$ Ries D uñ angeflogen Silopi-Erts.

67 Eine ungarifith 10) und D baltiges Etuffichen.

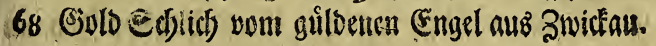

Broentents

\section{Die Sillber = Enke}

Scalten in fitif)

Jolgende 2urtben Gtuffen, als:

Gewadfene, gedigeme $5 \mathfrak{a} a \mathrm{c}=$ und Jes

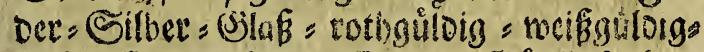

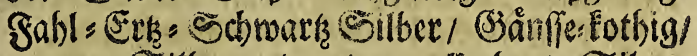
grau Sillber und andere allerband Sils berbaltige Plethen.

\section{Gdub = Rabe No. 3 . \\ II. Silber= Critze.}

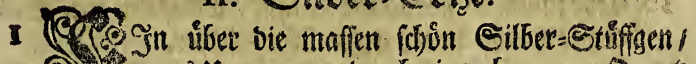

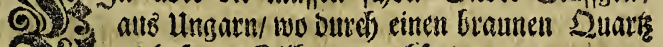
62: viel Jaan=-Eilfer gemadjifen.

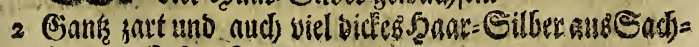
fen von Edjueclerg. 


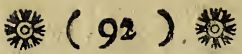

3 Eine gebiegene Silber=Stuffe mit vielem ausigetwact)=

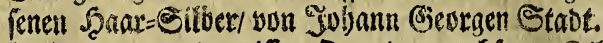

4 2lus einem gant weiffen Duar gewadjenen Cilber/

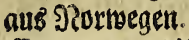

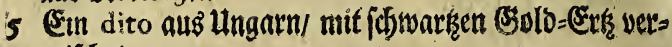
mirget

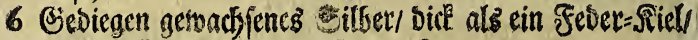
nus meiffen Duars bon Suneeberg.

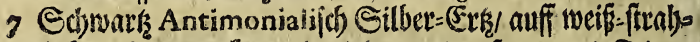

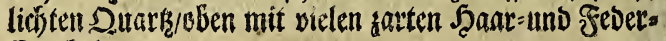
Ertic bele iset.

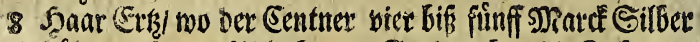
balt / auf Dem \$almboum/ Fundgrube / bie Zeche ges naint

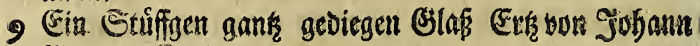
Givergen siadt.

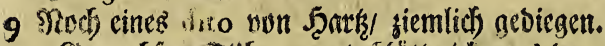

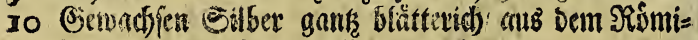
fden Ploler bon Yobatn Eeorge Etrot.

II 2 ge gefogenten gebiegeren eilber auff einem in roth. uno grat fobrnioben Geffein / von Jubgann Beorgen Etait auz der SRen- Jolyrez sMeffe.

82 Dito auf einem gelblithten Sdjitferidjten Beeftein, von vorigen Drthe und Gituben.

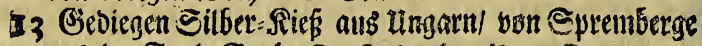
aub Der Fund = Corube Sanat Anthonii von Padua.

14 Faft gebiegene Eilber Etuffe/ von 2mbreas = SEersel bat funffifig Jabre ungebautet gelegen / nods eine Ders. gleidjen / und cine faft gants mafíve.

Is 2 ingeflogen gebiegen Sillber uno roth gatibig Errs/bort Johann Seurgen Stadt.

I6 Eine fofine rotbbriebige Etuffe / morinmen biel Eils

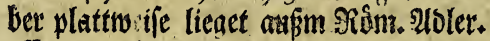

37 Eine groffe tuffe gewadbjen Silber auff Duarts mit

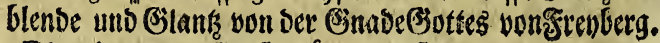

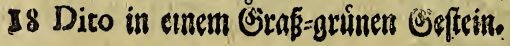




\section{翡 $(93)$ 新}

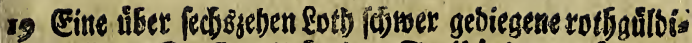

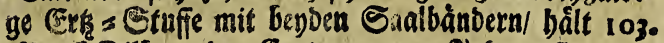
Siard" Eiliber/ Der Eenture / von Jobaun Gieorgen

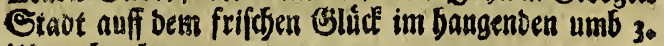
ubr gebrodsen.

20 Eint Epan / $\$$ ) Silber Mineral Armadillo genanntr.

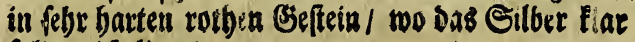
fplitterid) lieget.

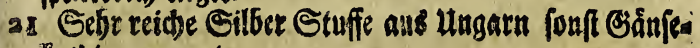
fothig genannt.

22 B̈edregen Silaß Ers/ Golobaltig/ von Egemnik aus ungarn.

23 Cin Stuiffgen mit Silbet=Er

24 Weiffer Spatb/mit gebiegen Silber ibberjogen als Slect) aus ulngarn.

2s Ein dito grofler uno reidjer.

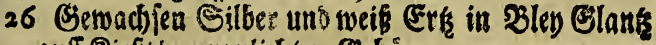

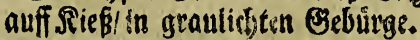

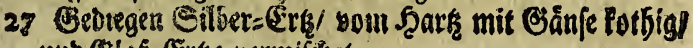
unb Bla Er Erke nermínet,

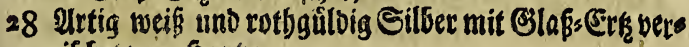
miffiet oon searfie.

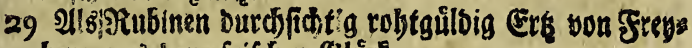
berg aus bem frifasen sslíte.

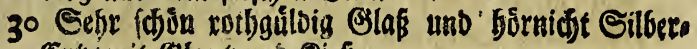
Ers/ mit BLlank uno Sief.

3r Stefflic) rotburilbig (Ers/ überall mie Granaten uno Nubinen glămkenb/ aus üngarn.

32 Eine groffe gediegene rothgutloige Silber-Stuffe von $10 \frac{3}{4}$. Potb/ ton 2lnoreas:3erge aus Det Felicitas.

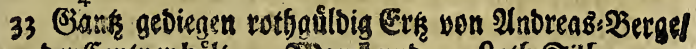
ber Centuer bilt 7\%. İard und 12 . Soth Silber.

34. Dergleidjon bon efen Dem Drthe aber gruffer.

35. Dergleichen aud bon Sarge.

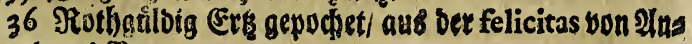
oreasisarge.

37 Cine fobue \$feiffetmeif getwadjfene rotbgutbige Stuffel uon pber Dem Drthe. 
38 Dito anders getwadfen.

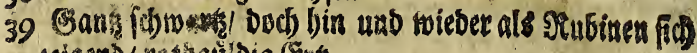

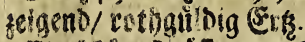

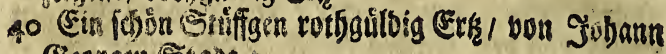
Gearoen Staot.

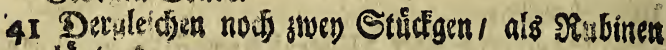
glânkeriot.

42 Stuffe Suark mit vielen angeflogenen Glaß ove boufrerberg.

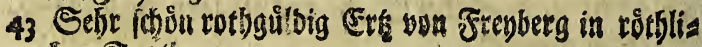
dien Epath.

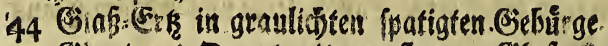

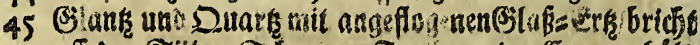
auff Der Silber E⿳亠丷冖巾.

2. Brardi Silber.

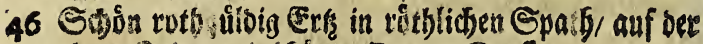
andera Seiten mir idjinen Dusn Stuffe.

47 Blafosern und gedieg $n$ Silter von frenbera.

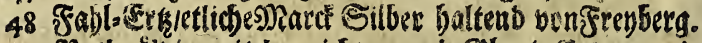

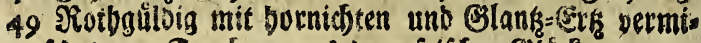
ichet von frenberg aus Dem friforen silute.

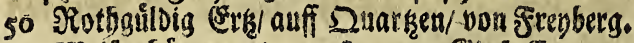

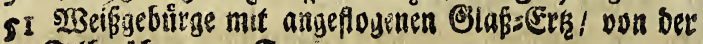
Silberiognur ju freyserg.

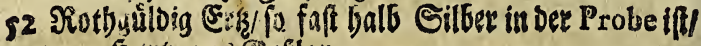

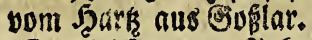

53 Dergleidjen yan Sobana Eeorgen Stabt aus Sachen.

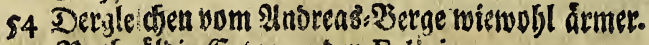

5s \$otbguilbig Erth yon Der Felicitas.

56 Dergleidyen aus Der (3rube Samfon.

57 Dregleid)en aus Der (S)

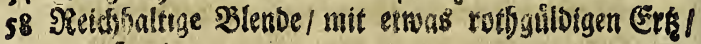
bum forthe.

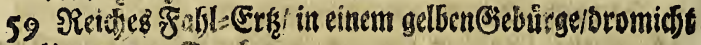
liegen/ non freyberg.

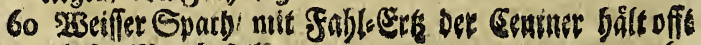
etlide s) sard Eilver. 


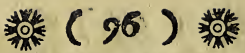

83. Dergleider aus Alixiwarie in Bapplanot.

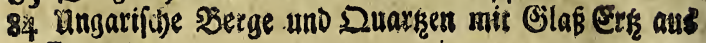
(Exemuirs.

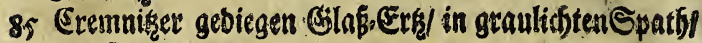
aus §ore=ungarn.

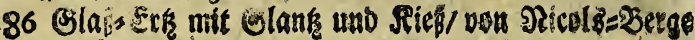
in Sobibmen.

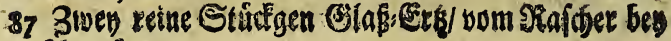
भintuáerg.

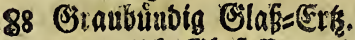

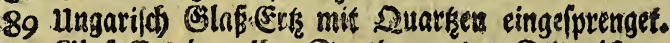

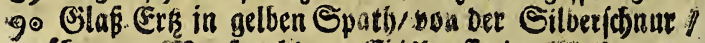

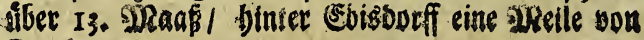
frevberg.

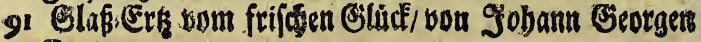
Etabt.

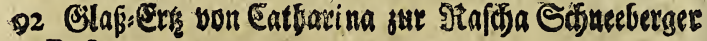
Refier.

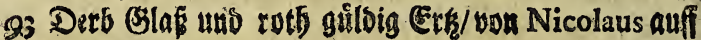
Dem Brande.

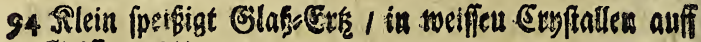
(se) iff aus ungarn.

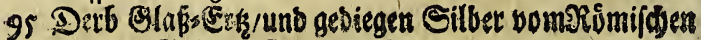
2liler/ zu Jobann Georgen Stabt.

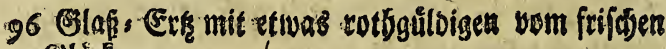
B!tute.

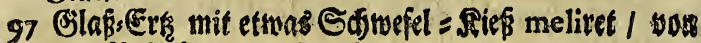
bem Malmbaum in Frenberg.

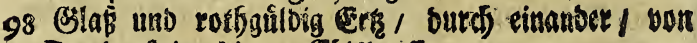
Setwenftein/ binter Ebisoorf.

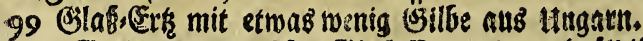

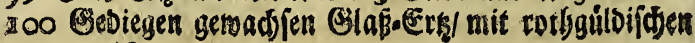
vermildst.

sot (a) \&̈laf. Ex(s/auff einet weiffen Drufe.

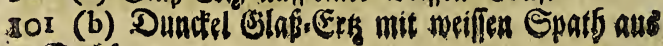
Sa中ien. 


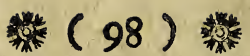

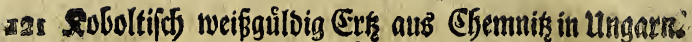

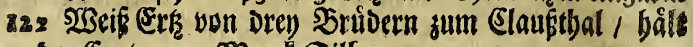
Der Eentner 2. Marde Silber.

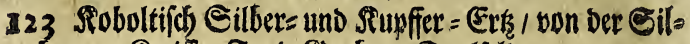

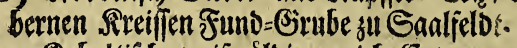

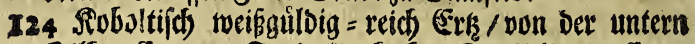

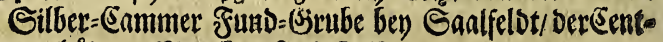
ner bált 1. Maref́ 8 fotl) Sillber.

125 Erobipeifigt Silber=Eris/mit rothgelben Spatt/uon getreuen Eifinot "u Saslfelot.

126 . Frenberger weisgelblidfter mit S广lant/ in weiffem Epath.

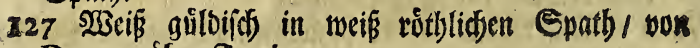

Donat, liber Greyberg.

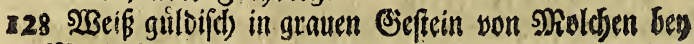

Marienbern.

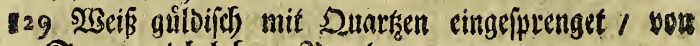
Sonnenivirbel beym Srande.

330 Dergleichen mit (Elanks/Epath) uno Rießs.

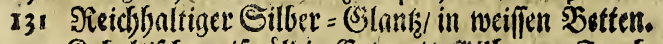

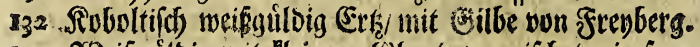

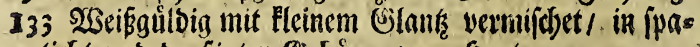

ticht und brufigten Gebuir

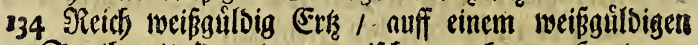

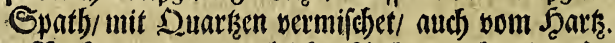

I3s Stocf) anders von gleicfer 2(rth/aus eben DemDitfe.

Det 6. Saften.

136 Cin anber Etuffgen mit gelblidfen Spatb und

Quarts vernifobet bod viel reidber.

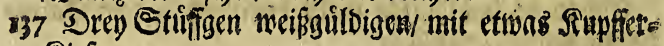

Siefs.

138 Fanbl Silber Erts/auff weiffem Spatt/aus ber

Sdjweik.

339 Fabl Ere in Spatb gepurnget/ won Edjarfienberg.

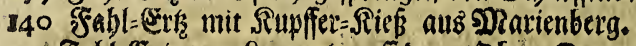

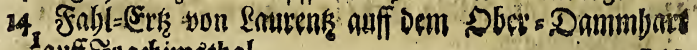
aufi Juarbimstbal. 


\section{耀 ( 99 ) ) 粼}

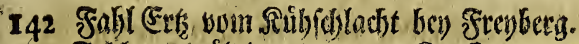

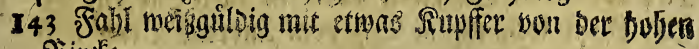
Situke.

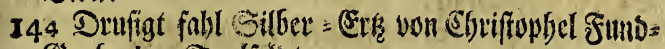
Gitube bey Salfelot.

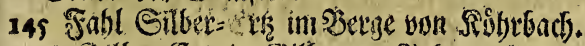

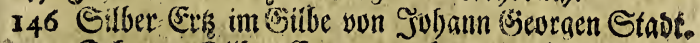

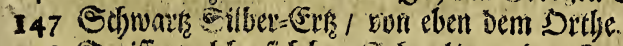

148 Speifie weldse fich ben (Sctjmelsumg Der Saalfelbio

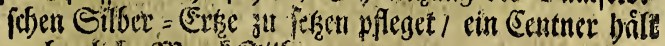
nuch etliche shat Eilber.

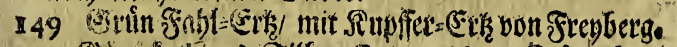

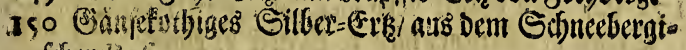
fojen Refier

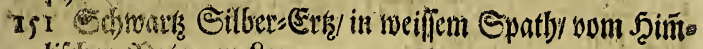
lifocn Sater fu Rangchau.

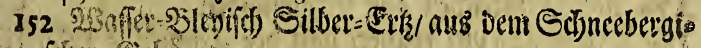
icjer Seburge.

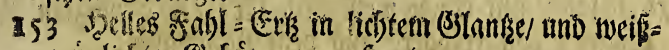

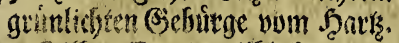

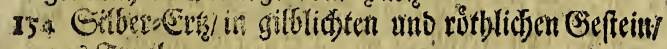
a

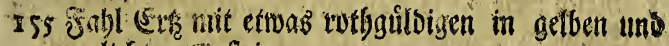
graulidjten seffein

I56 Dnndel Fabl=Erfil mit cingefprengeten Supfer= Riế bou Frenberg.

157 Gemein frabl= Sir won Franberg.

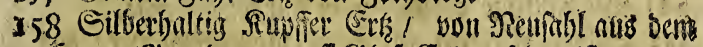

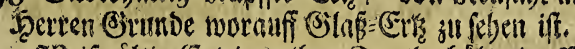

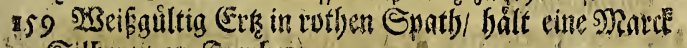

Siller/ uon fententerg.

160 Eine Etuffe von Sronberg / moran etwas groiegews.

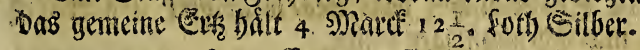

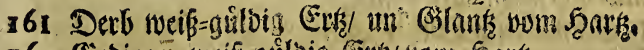

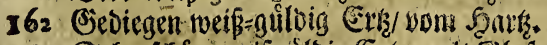

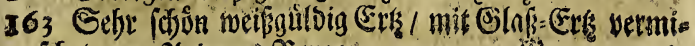

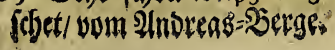

(⿻) 2 164 


\section{站 $(100)$ 新}

164 Dito vom Elautithal aus Dem alten Seegen.

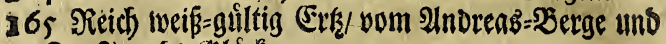

St. Jacobs=:Shict.

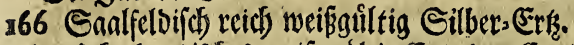

167 Dberbarkifiobes weif-gultig Ever/ ber Eentner bailt

2. Marté is $\frac{1}{2}$. Both) Silber.

368 25ei

Serge.

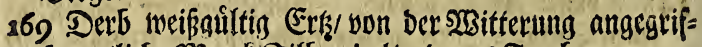

fen/ etlicbe Mincti Eilber balteno/ auz Frenberg.

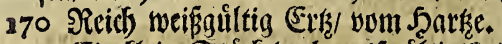

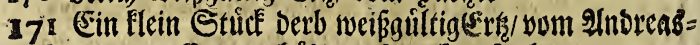

Berge/ ber Eentner bált 6. S) artê 8. Sutb.

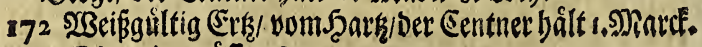

373 Dito cin grufferes.

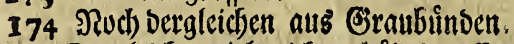

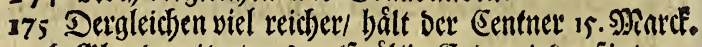

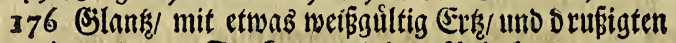

Duarke/ yon Freyberg aus Dem Palmbaum.

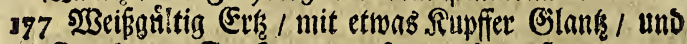

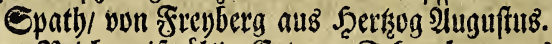

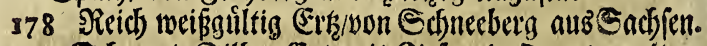

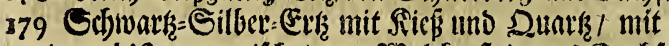

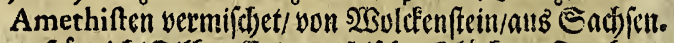
s.80 J̧ortnid)t Der 7. Tinften.

181 SBeifiniltige Stuffe/ unm Ilnorens=\$erge.

182 Silbet=Mineral mit \$erg = blau vermifhet / ats

Giraubuinien.

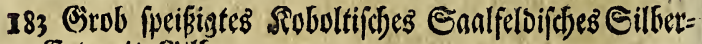
Err mit Gillbe.

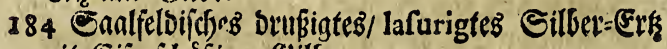

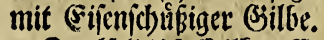

185 Enalfelbif Eilber: Erts.

186 Robolt-ppeifigt = gruin befoblugenes Saalfelbifbrs

Silbet=und Supfifrecerts. 


\section{橉 (102) 紧}

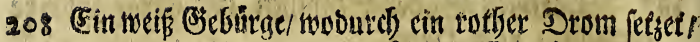

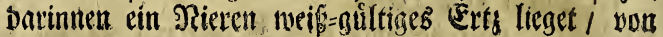

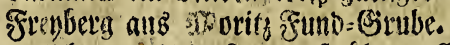

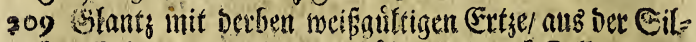

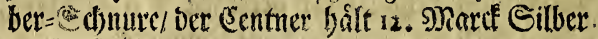

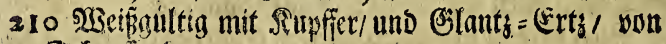
Estjurfienterg.

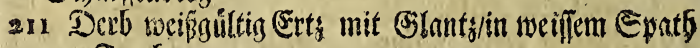
von Frenberg.

212 Silber Eitty / weifguiltig/ boun alten Ecegen boun

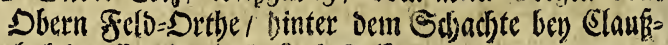
thal/ ber Eentner $17 \frac{I}{4}$. Qutl) silber.

\section{Der 8. Raffen.}

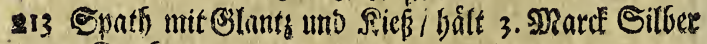
won Girub ferg.

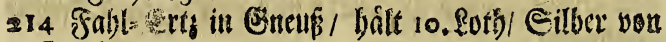
Freaberg.

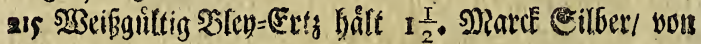
Frenbers/ aus bcem Palm-23aunt.

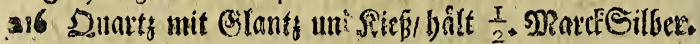

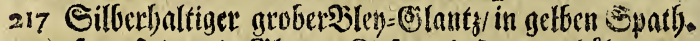

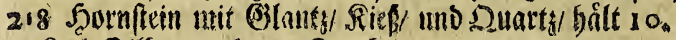

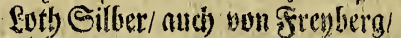

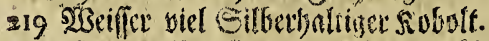

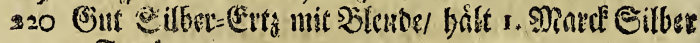
von frenlerg.

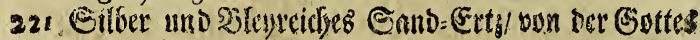
Gaabe zu Silmethnt.

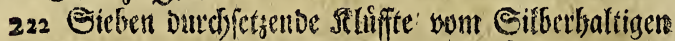

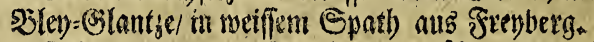

223 Edjyants uno wei (S) ber/ wen Ssenterg.

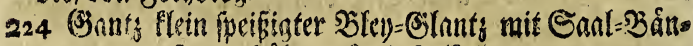
Deru/vom . Gants baitt 8. \&uth Eilfer.

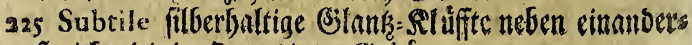
ftretidentot in Duarkigten Belvirge. 


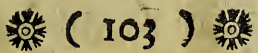

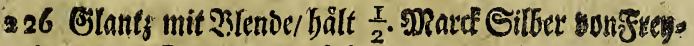
berg/ bon Sonnen=2Sirbel.

227 Sirof uno fleiner Ġlants / bålt ein Maref Sillete von Freenberg aus Dem Siálber Erantze.

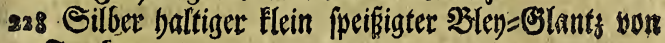

Frenberg.

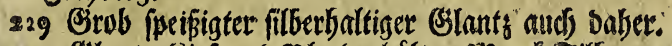

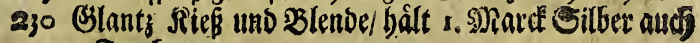
von Frenberg.

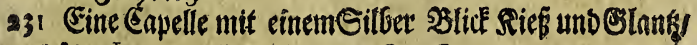

bålt $\frac{I}{2}$. Natadf Silber von Freyberg.

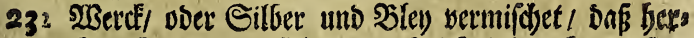
nad) in Form Der Ejlette im Srieb=Dfun abgetrieber

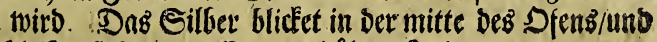

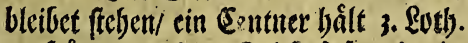

233. Scârte aus bem Trieb=Dfen / Darimnen Da Gilbet abgetrtieben wirb/ Gált viel $\mathfrak{s}$ len.

\section{Dritten: \\ Die 2 len = (Erke \\ 2ปllక:}

Gediegene, tociffe und allertband farbis

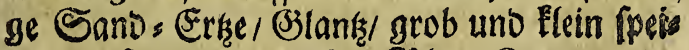
figte/ und fonften Blev = Snos.
fpidite Stuffen.
III. SZlev $=$ Srk̨e .
Der 9. Zlaffen.

I Thajefoun gebiegen / als sweige getwadjenes 5ley! 8) auf ciltem getben Beftein / wun Esfinets berg.

22 S3ei SBlen von freyberg.

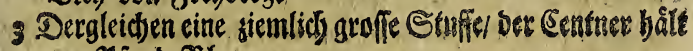
80. Pfund $\mathfrak{2 l e y . ~}$ 


\section{粼 (104) 耀}

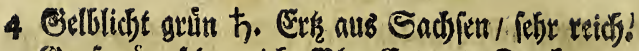

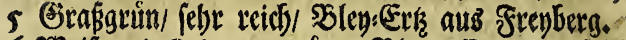

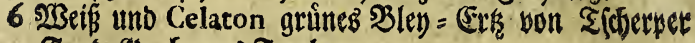
Fund=-ઉrtubeaus Srenberg.

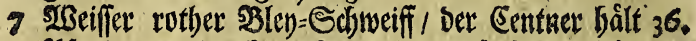
Spfind utmi surcd) deftillation verfuctjet / giebet cin

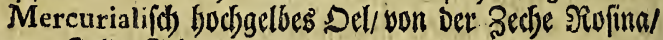
jumm Bellev:Felae.

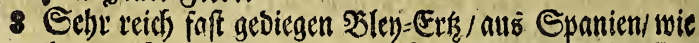
lauter lánglidbte Diere-Guler bâlt Eilber und Rupfer.

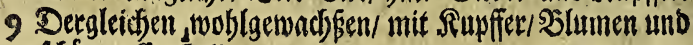
ichósuen Eryjtallen.

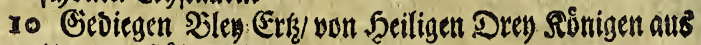
unnaarn bâlt Ssub unb Silber.

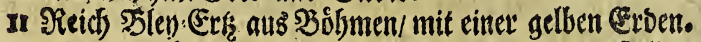

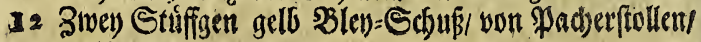
aus̉ Unyarn

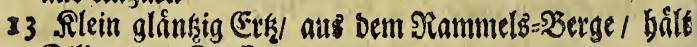
Eilber unb Sirpfier.

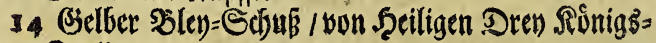
Stollen auts ungarn.

Is Dergleiden won ber Sceiligen Dreyfaltigleit etwas grófier.

16 Bivubglinnthig(En

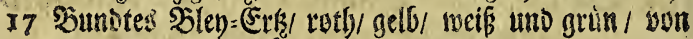

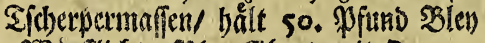

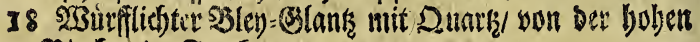
Birdefe/ in Frenberg.

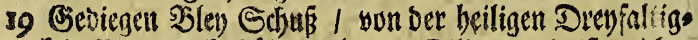
Beit \$Bergwertíl bâlt Sold und Silber/ und iff reichet in Biolhe.

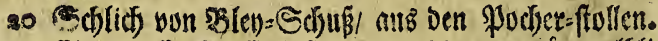

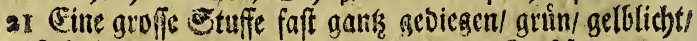

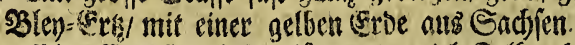

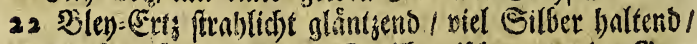

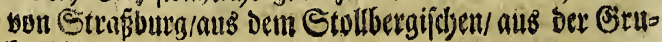
be sleonora senannt. 


\section{(105) 嚙}

23 Eine grob = glintstige fert reicfe 2 bley = Stuffe you STats.

24 Dito Elcin-fpeifigt und ármer.

25 Dito wab unterídjieden.

26 Saalfelbifher silen=-Edieffer.

27 Ein artig gewadofern blen=Striffien/welldes wie laus ter Gilieber won einer Retten aubfiethet.

28 şlen=:Stiffigen aus ber Sojweit?.

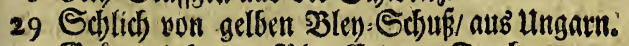

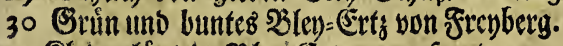

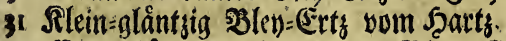

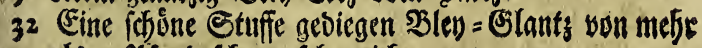
als 1. Pfind fodmer jefrer reich.

\section{Der 10. Taffen.}

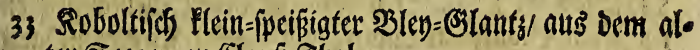
ten Eeegen ju Elaub-shal.

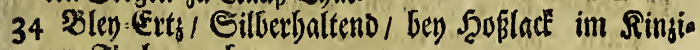
ger Sbal gegrabert.

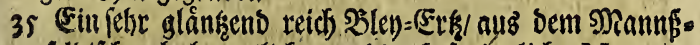

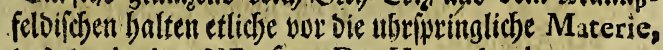
Des Agricola. NB. Şert Dr. Hattenbach.

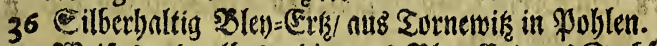

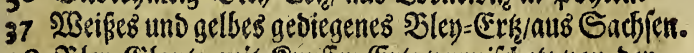

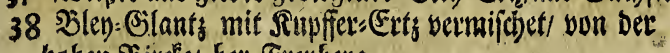

bobsen Sirtue/ ben frenbertg.

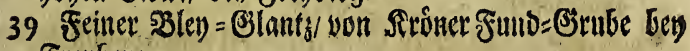
greenberg.

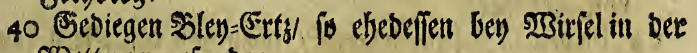
sisetterau gefiunben.

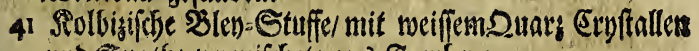
uno Epatb/ vermif(d)et/ auş Freenberg.

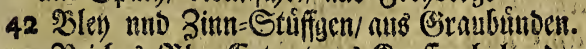

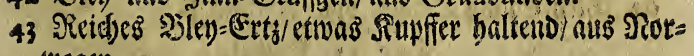
wergen.

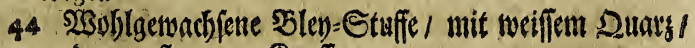
und angeflogenem Rupffer. 


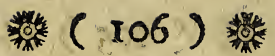

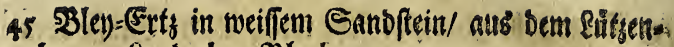
burrger Ranbe bey silenberg.

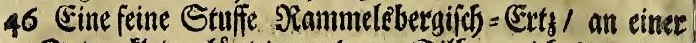

Epitze flein-oläntzig/ und an Sillber reidfes/ an Der

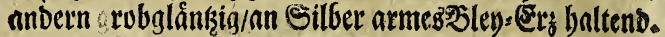

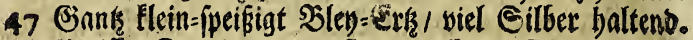

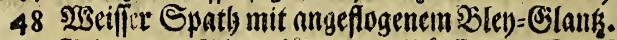

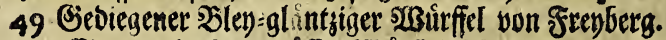

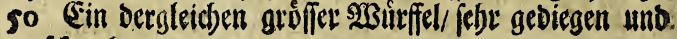

idswelfr.

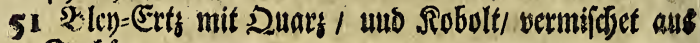
Sacbjen.

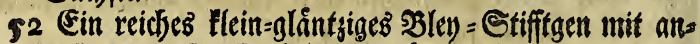
geflogenen Siupfier=SBlumen/bălt Silber.

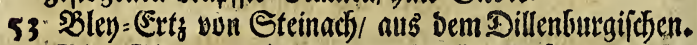

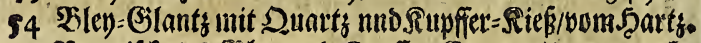

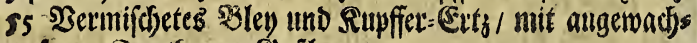

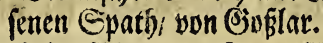

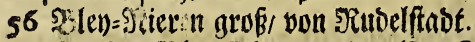

57 Derber $\mathfrak{B}$ Len=-Silan:/ mit gelben Suinder angeflogen/ vour freenberg.

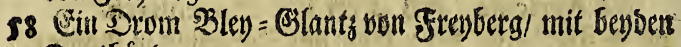
Eaalbinuern.

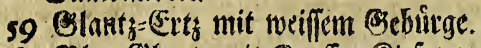

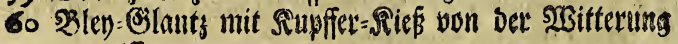
angcyrifen.

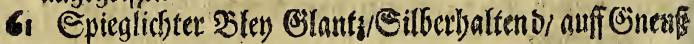
von Frenbera.

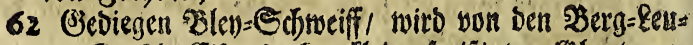

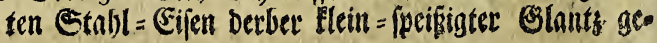
nannt.

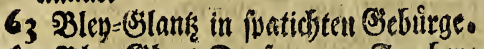

64 Dlen=:Siank Drufen yon freuberg.

Der II. Saffen.

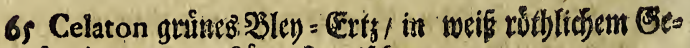

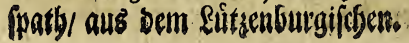




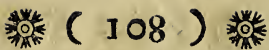

Shietvon liegt in Rafen No. 20.

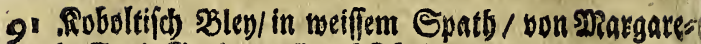
tha fanto, Girube im Elauf S S fal.

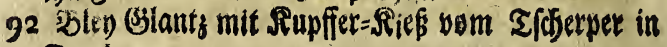
Grenberg.

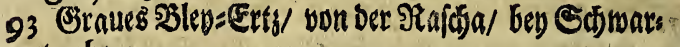
tsenberg.

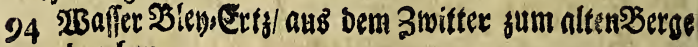
gebrecten.

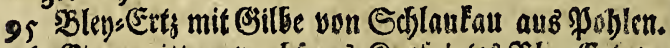

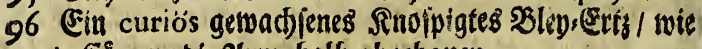
ein Cirper/ Dee Atrme balb abgefjauen.

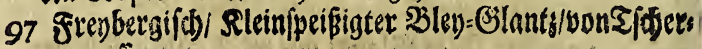
permafien.

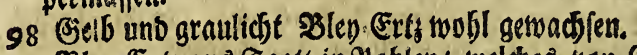

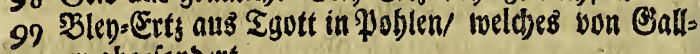
mey abgefonbert.

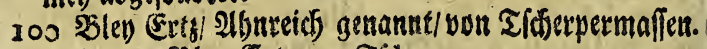

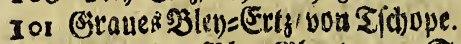

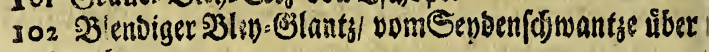
Frenberg.

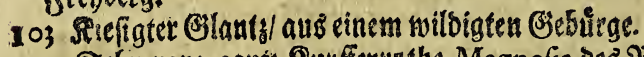

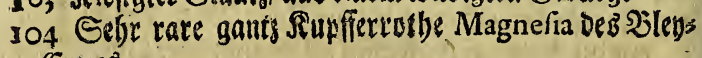
sitzes.

Ios Speribigter Elants von Naraaretba zum (laufitjal. yos Eeoiegen Silen=Erts aus Sadfent.

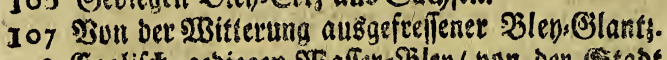
108 (Eagliff) gebiegen $25 a$ ffer-s3ley/ bon der Stabt Darby.

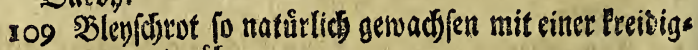
ten Materic áberjogen.

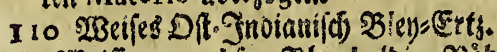

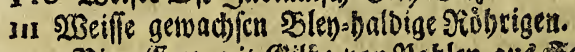

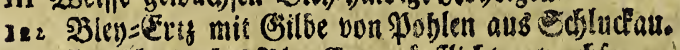

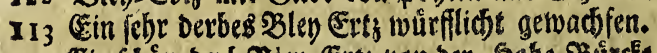

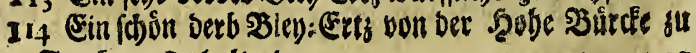

Frengerg D balteno. 


\section{紫 $(109)$ 粼}

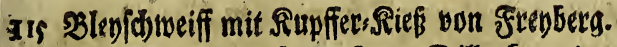

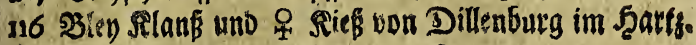

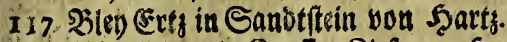

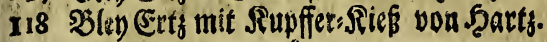

119 Sleg Certs mit grime.

120 Bler in einen weifen Ginter von Frenberg.

121 D. Jaltiges 5ley Eets in rótblichen Spatb yon ber Sircte su frenberg.

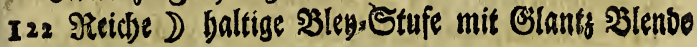
in Spat5.

\section{2ierdeteng,

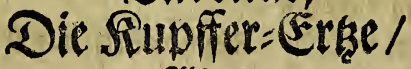 \\ 2!'B:}

Gediegene, gewoadfene $\mathfrak{R u p f f e}=(\mathfrak{G l a} \tilde{B}$, Sundtes Supfer = Erkg/ \&ajurigte audb Neus foblif be aus os crementiret / und fonften allerband o \& Erge, Stuffer.

\section{Supfier = Erket. Der 13. Ziaften.}

I Ges Effir gebiegen getradjen Rupfier/als cin ats

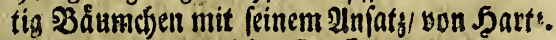

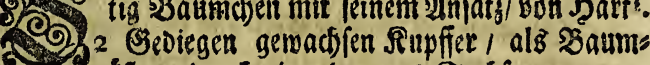
áftgen burds einander aus Sacjeer.

3 Ein groffes Stiut gebiegen getwadfen Rapfiex / yon Sdjnecberg aus Sadjen.

4 Taponifo Rupffer fefre idoón.

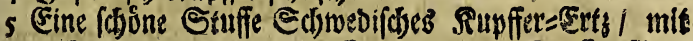
Ditern gewadfenen Tupfer / aus Dem Rupfers: gen/fey ber Stabt Taloc, 27. von Stoctbolm.

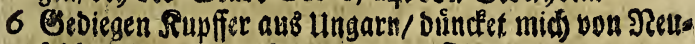
fobl gu feyn weaen feiner artigen Figur.

7 Ein aebiegen Rupfier = Stifffien aus Sdjweben/ wie Rupfierorafb Durdjetnander gewadjíen. 


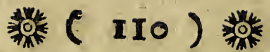

8 शrod sin anbers von Scjneeverg in MReifen.

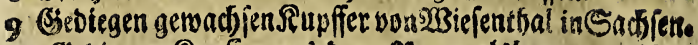

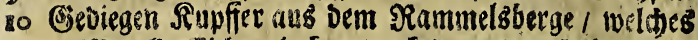
an 1 Retall = Eidben (aber an fein alber scolts. ) uno פoobianifieffet.

II Derb gewadffen fupfier in melfiem Duarts/bon Sints bei Saalfeldt.

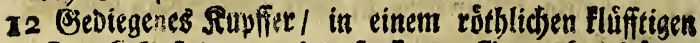

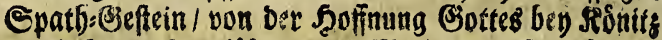

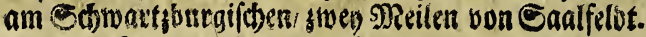

13 Dergleiden von eben Dem Drtbe.

14 Ein lobin Stuffetein Tupfer - Sdjieffer/mit Conchiten in Dicferm Seiés.

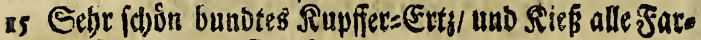
ben fpielenvipon frenber:.

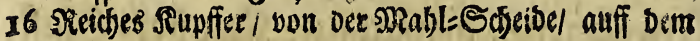
Wrefterwaloe.

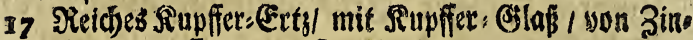
nebetger; auff Dem 23 efter=233alde

I8 Reidje Frametenberger Rupfer Braupen blair von Farbe.

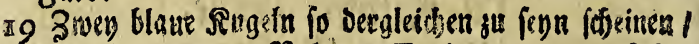

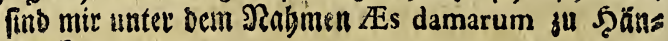
Den fommer.

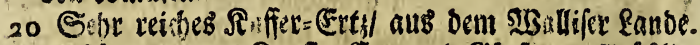

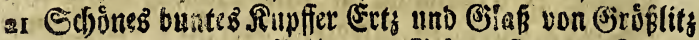

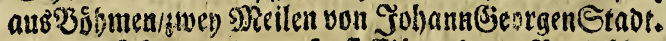

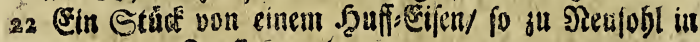
ungarn ju Supffer morben.

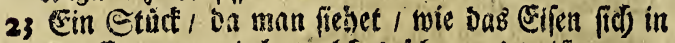

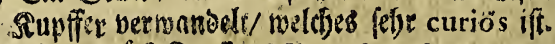

24. Ein Stúc Rupfer Mineral aus Ungarn/ woraus Das̉ seumffer überall bid wie baar beraus gefproffen.

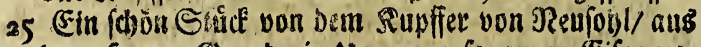

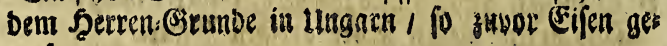
wejen.

26. Dergleiden in fleinere etriften 


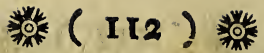

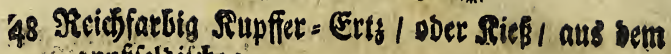
waannfiseloilden.

49 Supffer Mineral mit etwas \$ley vermi (d)et। aus

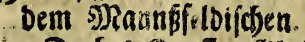

so Derbez Surpfer: B̧las.

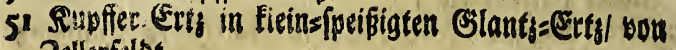
3ellerfelos.

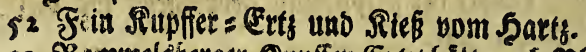

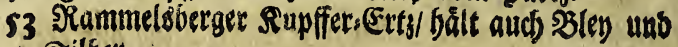
Silter.

54 Derbes Supffer (Evt ober fo gemannte Sunft vom

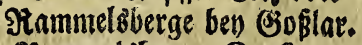

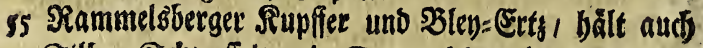

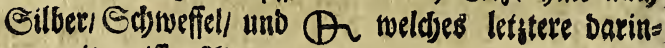
nen mit weiffen 2 been su fefber.

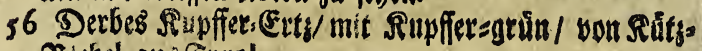
Dichel aus Syrol.

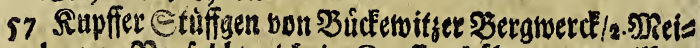

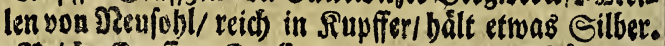

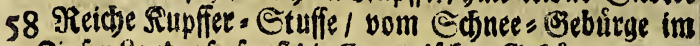

3ipjer:Ranbel fo fouff bie Carpati(d)en Geevuirge genens net werben/fält Ssold uno silleer.

59 Supffer Mineral aus Ungarn/in berGiegend SReurobl. 6o Rupffer Mineral bon Neufobl aus bem Scrter

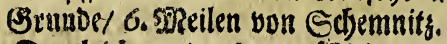

6r Dergleicter etwas untert (ibieden.

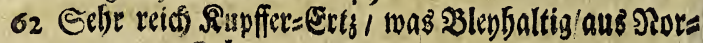
megea von edsonen.

63 Rupffer Eirt/ mit weiffen (Eifenffein vermenget/ vom

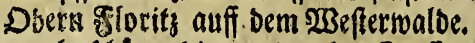

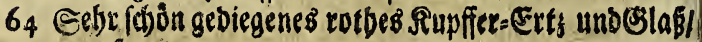
vom Scatts.

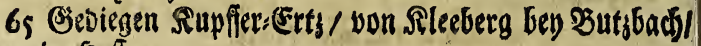
in Şeifen.

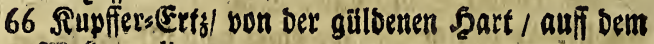
weffermalde.

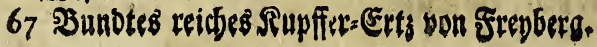




\section{背 ( II4) 耀}

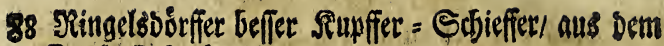
Sinnf: Schadte.

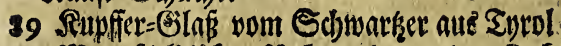

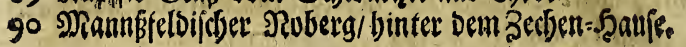

Der 15 . Raften.

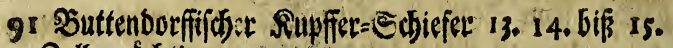
Zoll mácbtig.

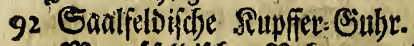

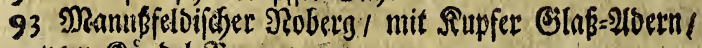
yom Silindel=serge.

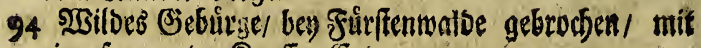

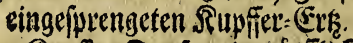

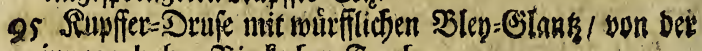
jungen bolsen 3 birle bey Sirenberg.

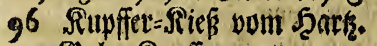

97 Baalyss Pupfere.

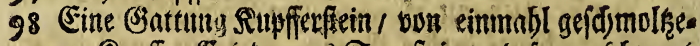

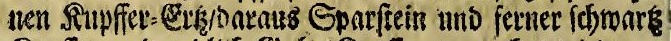

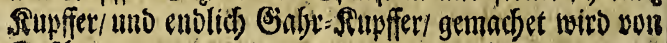
Goitlat.

99 (Eine andere 21tet)/ won obigen Spabrffein.

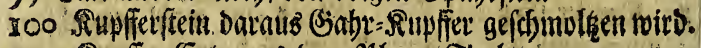

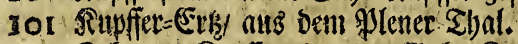

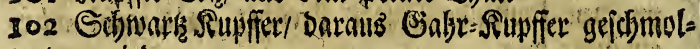
ken wirb.

103 Edjlacten son Siable-Rupfier.

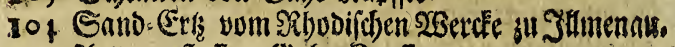

105 Intig geffuiten Babr-siupfer.

106 Nupfiez=-E colfact:

1 os SSseidese Supfier-baltende Esfinder.

108 Soupferfifein.

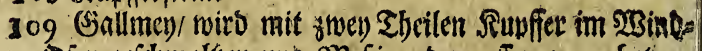
Dien gefibmul(zen uno greeging Daraulfer gemadjes

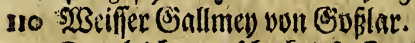

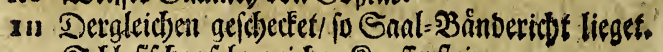

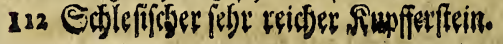




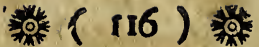

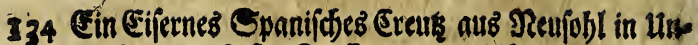
garn/ po in bas befte Siupfier verwandelt tworben / aus Dem Bergsobiunnen/ Dns alte Cement gennnnt 1689. genoument.

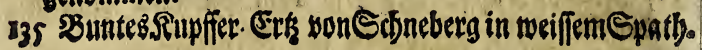
Der 16. Eafien.

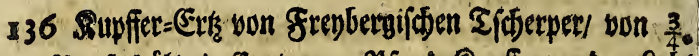

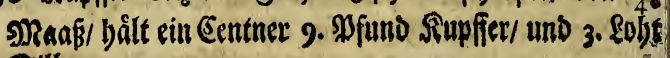
Eilber.

137 Gabb- Ruyffer/ yon greevenffein.

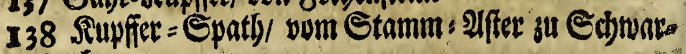
- kentburg.

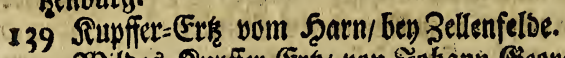

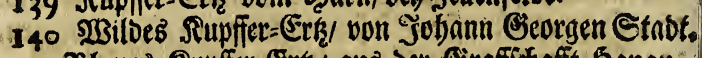

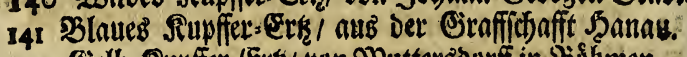

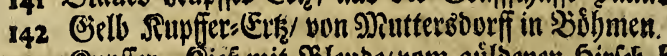

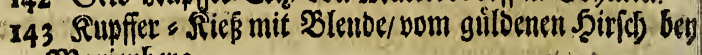
Marienberg.

144 Supfier Sief won Jobann Eevorgen Etabt.

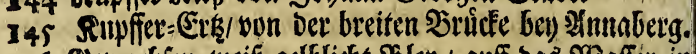

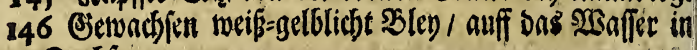
Sachien.

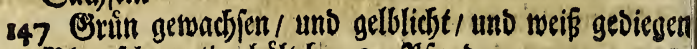

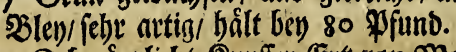

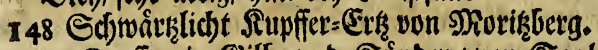
149 Surpfier in Eillbe und Einders / von Saalfelbo. 250 Ediwartes Supfier.

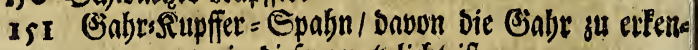
nen/ wenn er/ wie Diefer runtzelidet iff

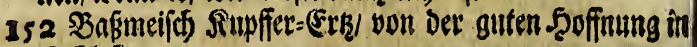
Sdilefirm.

s53 Eelyr reidfbaltiger Supfiete: Sdicfess.

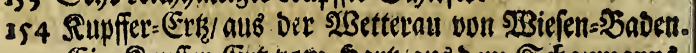

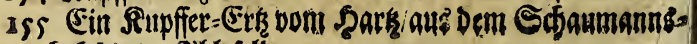
thal binter Shblefeld. 


\section{龉 ( \\ Jünfftens, \\ Die Eiren=Steine/}

als:

Gediegen, gewwadfene SIafs= Roppfe, (Ei:

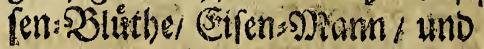

bergleicber.

V. Bifen=Stuffen.

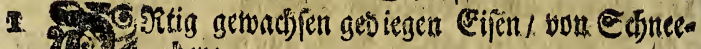
GE

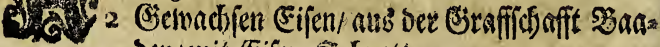
Den/mit Eifen=Estrott.

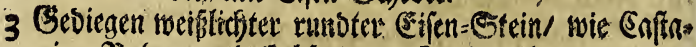
nien/ Sovfnen und Erbien/ aub eben bem Drte.

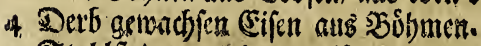

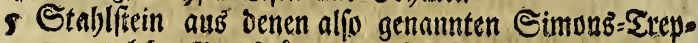

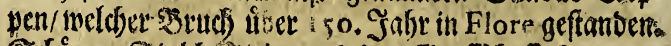

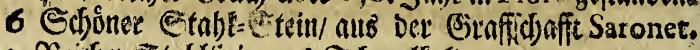

7 Seictser Etablfiein aus Edfontalfalben.

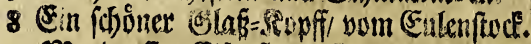

9 Marborfer Eifenftein/ frebet wie Edjeot/ bey. Stoma buts in Seeferen.

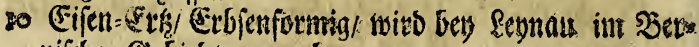
nifdjen Giebiebte gentaben.

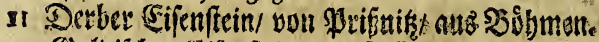

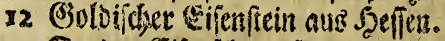

13. Derber Eifenfobrot/ /o aus bem vorigen Stein gefallet:

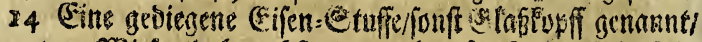

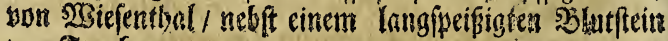
ban frensarg.

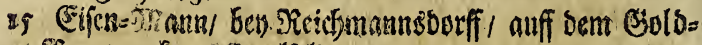

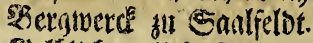

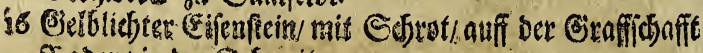
5abrn/ in ber edjweirs.

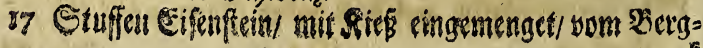
inert" 


\section{橉 (ro) 形}

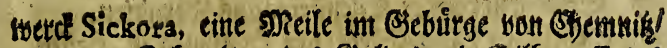

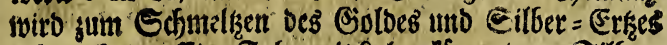
gebrautdset. Einte Fufut mit Robn tómmt so. Silber. Ģrofoten ju fteben.

18 Siúpfiferichter Eifenftein yon Dlferobe.

19 Şatḩigtet Eifenfftein.

20 Eifenffein bey swarienberg/ 80. Pfund Eijen balteno:

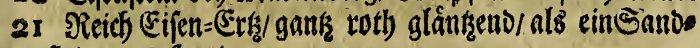
ftein/youm Şar

22 Sdullenburgi

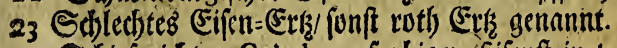

24 Sdfieferidfter Celadon-farbiger Eifenftuin/ aus Boheimien.

25 Gifen-2bluttbe aus ungarn.

26 Siother (Eifenftein in woiffemepatf.

${ }_{27}$ Эibler Mingnetffein.

28 sieicher Eifenftein aus ber SteyermardE.

29 Eraunfpatigter Eifenfein/non Rinuenftein.

30 Blutfiein unit meiffem Eifenffecin vernifjet/ wan-bet 3orgen an \$ar

3r Ârtiger gelber Eifemfein aus Eadjen.

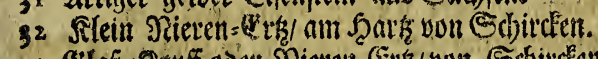

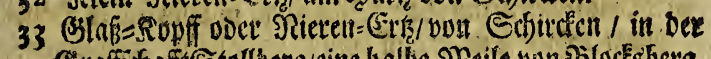
Eraffidyaftestoll berg/eine balbe s)eile vin §locksberg.

34 Dito curiös, als ein Priapus geffiltet.

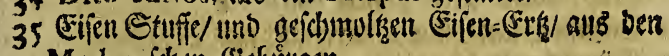
Modenidjen (sebuirgen.

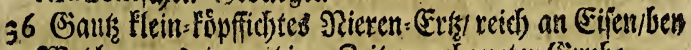

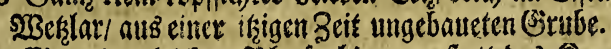

37 Eben Dergleidsen Slenfarbig / an fiatt bez Seupfers lange Streiffen babend/ weldfe glcichjam Drgel-\$peiffen abbilben.

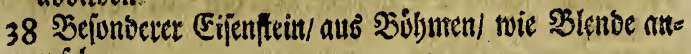
jufelten.

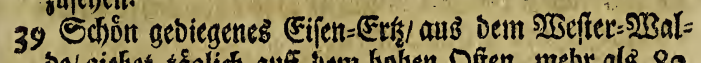
De/ giebet táglid) auff bem boben Dffen mebr alz 80. Centnet. S马 4 $4^{\circ}$ 


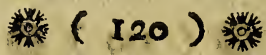

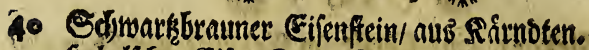

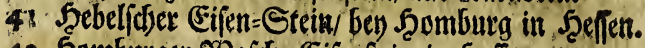

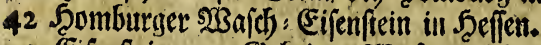

43 Eifenftein von Eefrrigb = Wुstefen / auf Dem guitliden Sergleide vom Jark.

14 (Eroforbiger mit gelo= und roth eingejprengetert (Eipenfiein aus $\mathfrak{B}$ śbmet.

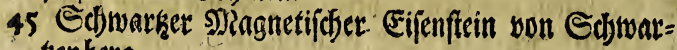
kenberg. 46 Eiiens=slietfe cuts Rärnbten.

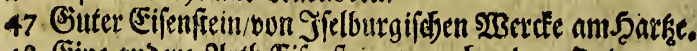
48 Eine andere 2(rth) Eifenftein/ von eben Dem Drte.

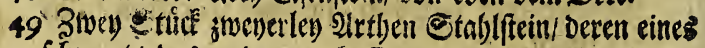
fobmarkil Das anoere rotb Ever genenuet wirb/aus ber Eraffichafft Sarunet.

so श?och cin anber ziemlid) gebiegener Eifenf(tein/son eben Dem Drte.

91 9)agnet-Steir von Schwarkenberg.

5.2 Neicher Eifenffein/ mit Siupfer vernifidset/ und anges flogen/ von ubigen Drte.

53 Dergleidjen etwas unterfobieben/ von Slienburg.

54 Eifenftein bey Dem Rloffer Şenna gegraben.

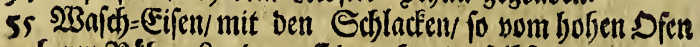

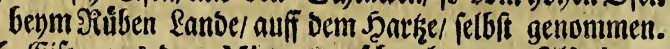
96 Eifen nůs Dem Mineral gefdbmolken von Jlfenburg.

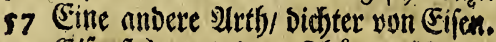

58 Gifenftein aus bem Shturingifben.

59 Eifenftein/ fo fthon cinmall int Feuter getweien.

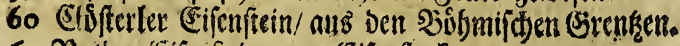

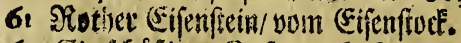

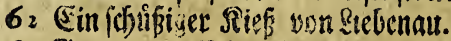

63 Car artige (Eijen=Eshlace.

64 Indere / auts ber Sdjweik/ als Den Sarunetifjen Dfert.

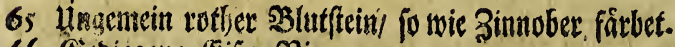
66 Bedoiegene Eifer=:Aieren. 


\section{站 ( I 2 I ) 赫}

67 Eifen = Mineral aus bem 20ener (befbietbe.

68 Eifenferin/ vom netigebolinen Sinde/ bey 230 olden:

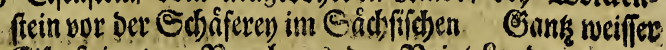

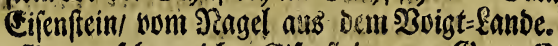

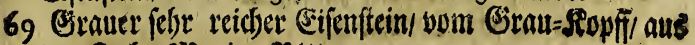
Der Zeche sysarien=Bitte.

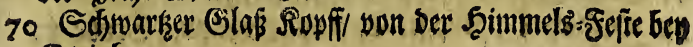
Etcinberg.

7r silaue (Eijen=Sd)laden von Sllemburg.

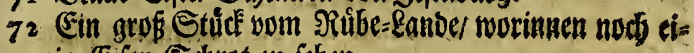
nig Eilen=Edbrot zu fében.

73 Эietider Edfweizerifher Eifenftein.

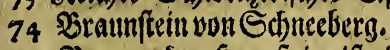

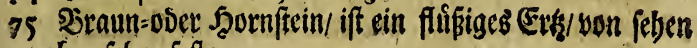
aber felfor feffe.

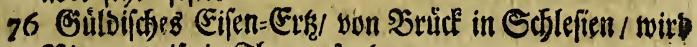
Nierenalueife in Shon gefunben.

27 S2Beifier Eifen=Stein aus \$areuth.

78 Eien- Rugeln von Tupplis.

79 Drey bejunbere 2letben Eifenftein/ yon verjobiebenen Suthen in Snchien.

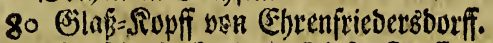

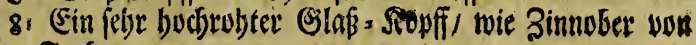
Fantie.

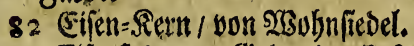

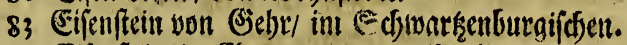

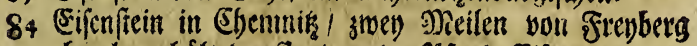
gebrodsen/ bält Der Eentner 64. Pfund Eifen/ und ein balbes Roth (Siblo.

85 Silciner Bilapisopfi/ won Roinig ben Saelfetbt. 86 Eijenfeil von Der \&unge/ aแร bem Altenburgifben . 3 twitterffuct.

87 Eifenftein von Sornemit?.

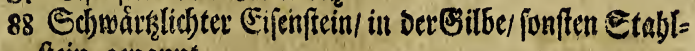
frein genamt.

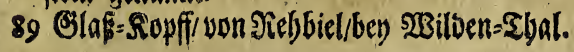

$$
\text { D. } 5
$$




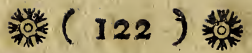

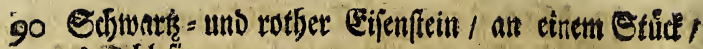
तแrs Sdilefien

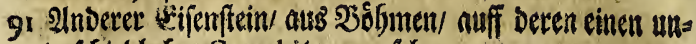
terfófiedlidfe Conchiten zu (eb)en.

92 Eifenffein von Sornewirs.

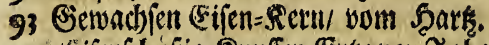

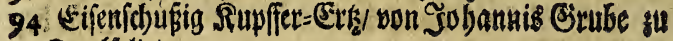
Eaalfelot.

95 Edjone Eijen=sliutte nuts Snrol.

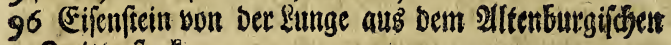
Smitterffode.

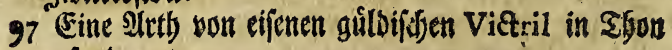
gefituben.

98 Siecther=Eifenffein / bin uno wieber als Sinnobet ant jufthen aus sacjien.

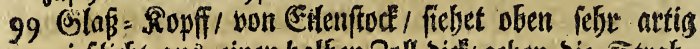

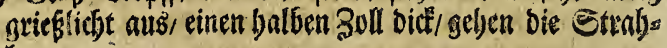
Ien an.

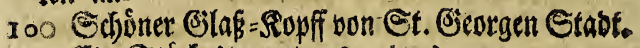
zor Cin Stud dito etwas anderB.

102 (Ein reid)er Eifen Elinber.

so: Sin febr reidjer wifien Estein von Eilenftutet.

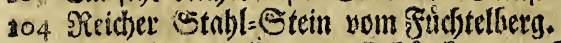

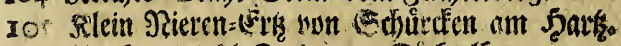

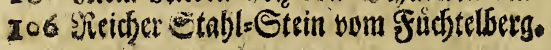

$$
\begin{aligned}
& \text { Sedtifent, } \\
& \text { Sinn Stuffen/ }
\end{aligned}
$$

श्राइ:

3imn = Craupen, Buitter aud $2301 f: 5$ $\mathfrak{r a m} /$ uno Dergleichen.

\section{Bint = Ertse.}

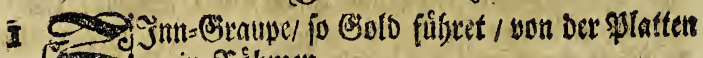
in soifthent.

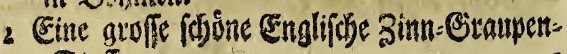
Stuffe. 


\section{粼 ( 123 ) 数}

3 3wo bergleidjen fleinere.

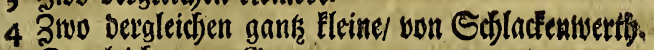

5 Dergleithen won Giener.

6 Eine fobine groffe 3inn=Braupe / aus Sachfen.

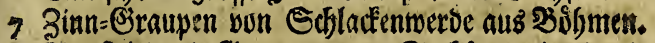

s äinnfetin uno Braupen/ aus Eachjen wic cin Ame thiffen=Mineral anzufelfen.

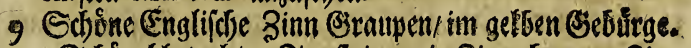

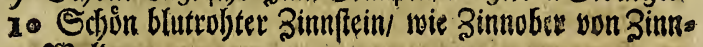
खBalloe.

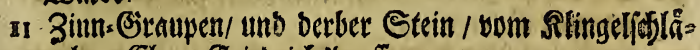

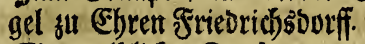

12 Gine gelblicjte Drufe/ wornuff einige 3inn= Craupen fificen von Sdjlactenneraloe/

13 3innftein von 3íbeliz̧.

I4 Zinn=-Sraupen und berber Sinnffeitu/aus bifimen. 25 Dergleichen nit Amethiften uns Spath vermenges.

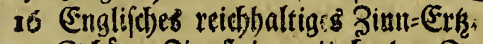

17 Sthiner Zinefferin/ nit beyben Saalbânbern: von Edneebern.

38 Reicher Sóddffifheses Zinnffein / mit Graupen.

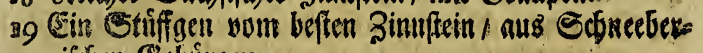
gilfhen Bebuirgern.

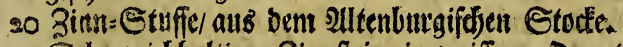

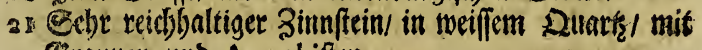
Granpen und Amethiften.

22 Cine frosine groffe Stuffe Bimnftein / und Giraupen/

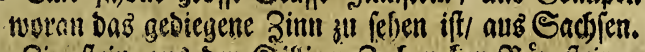

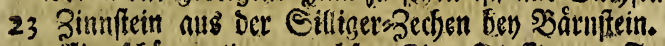

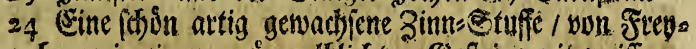
berg/ in einem grin geiblidiften Beffecin/ wait weiffert Ries.

25 Zwoen Fleine Etria obje Sief.

26 3inn-3witter yon 3oberis/ bey. Marienberg.

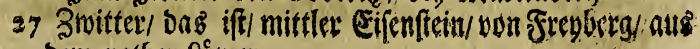
Dem votben fóner. 


\section{然 ( 124$)$ 橉}

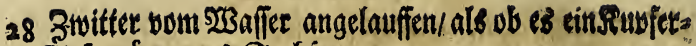
Sieié wionire / aus Sachjen.

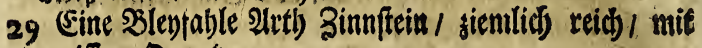
meifiem Duart.

30. 3mitter in 23 eiffenffein/ you ber 2utue.

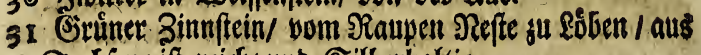
Sadjjen/ if reich/ und Silberbaltig.

32 Besiegener 3innffein/ mit graullibften Sefferin / aus Sólmen.

33 Sd)marker 3 mitter/yom 2 Altenberge aus bem Stodelel wortunter sibismutl) ift.

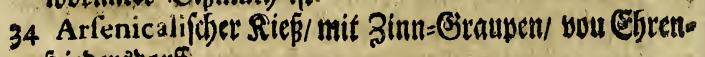
friebersbourf

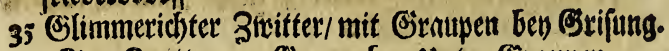

$3^{6}$ 3inn=Zwitter yon (Sener/ Gey) Unter-(Sraupen.

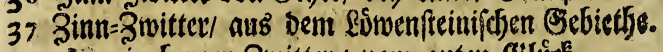

38 iilicarienberger 3witter / vum guten Blicte.

39 siother 3mitter von ber 2une/ bey Frenbertg.

40 Dergleichen roth und weiß// aus 2ovbeimier.

4' 3inn=3witter/won Sdflette/ been 2lnnaberg.

$4^{2}$ edneeberger 3inn-3witter 1706 . anbrídigh.

43 3innffetin uno Graupen in brausem Spath) bey PRa venffein gebrodsen

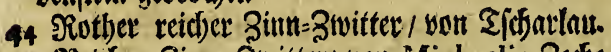

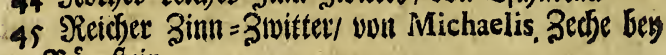
Bâtrnftein.

No. 2.

46 Silabrer graupiger 3mitter / in gelb-beaunen Epath.

47 Gin atberer flabe graupiger 3witter/ bon ber Sofa.

48 Эiotbe 3itn=Giraupen/ auff getbem Spath.

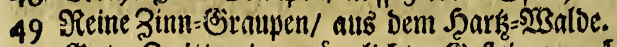

so (Suter 3mitter/ in grumulidbten Ejeftein/von eben bem Sitthe.

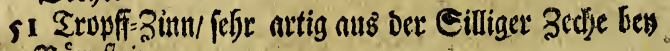
Bárufftein

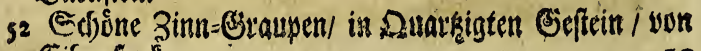
Eibenforte. 


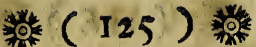

33 Sinn = Graupen von 33 åbrenfels.

\$4 3mitter in Sandftein/ won Jobann Gecorgen Stabt.

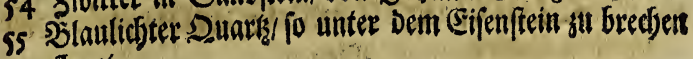
pfleget.

56 Sumbten 3witter/ aus bem Iltenburgifoben Stodfe.

S7 Dunctel rothe Sinn=Graupel won 2BSiejen= Shal.

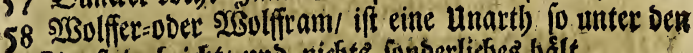
Sinnfecin bridtet/ uno nidjts ponberlidseb balt.

\$9 Sinn=-Siraupen/mit etwas gebiegenen - Bitn/ in Amethiffen/ von Srblactenualbe.

60 . Bwitter in rothlicfen Beffein / non ber Singel. Gi Soboltidster Zmitter/ bey Sdjneeberg ber.

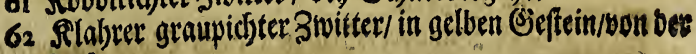
Sula.

63 Zwitter aus ber Sus an ber Beche jum B̧ânffein.

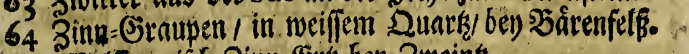

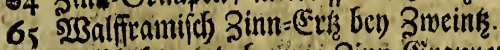

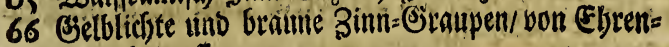
friebrid)sourf.

67 Silabr grauptester und glimmidbter 3witter / von Sdjeibenferg.

68 SNuber reidjer Zinn=3 wifter/won 3icherlau.

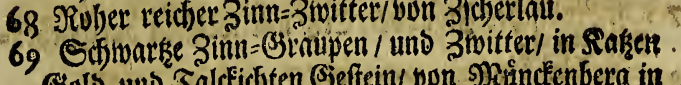

Gols uns Talctidten Geftein/ yon s) Sindenbers in Sólbmen.

70 Ingeflogene Zimn=Grautpe nuff einer Ergffallen Drufe.

71 ?eicher Ztwitter von Der Bottes Sabe.

72 Eine Zinn=Siraupe yon eben Dem Drth.

73 Sifoirl mit ainn=-Graupen vom Gener.

743 mitter.

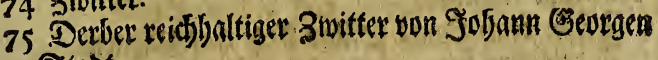

Stabt.

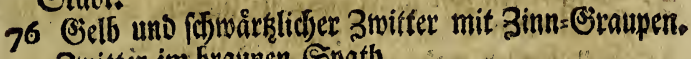

77 3mitter im braumen Epath.

78 Eine ếberaus fojune braitnlichte Zinngraupe mit iz.

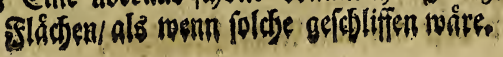




\section{粼 $(126)$ \\ Siebendens, \\ Quect:Silber=Stuffen/ Als:}

Binnober = Frk̨e, Itns anderet 茨 faltenoen 2uthen.

VII. Binnober -und Quect $=$ Silber $=$ Srese.

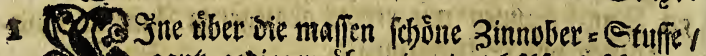

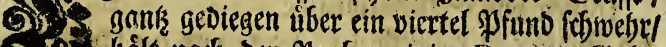

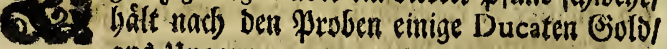
aus ungarn.

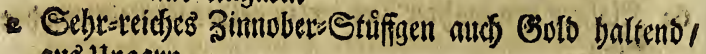
aus Ungarn.

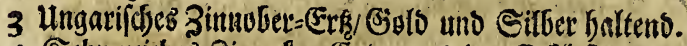

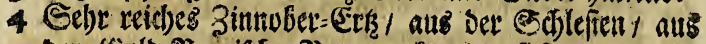

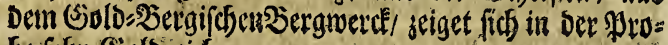
fe fefre Soldreido.

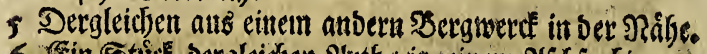
6 Sin Stud bergleidjen Irth $/$ in einem 2rfoffarbigen Eroenen Geftein fels artig.

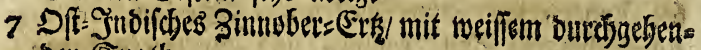
Den Spatb.

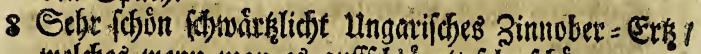
weldses wenn man es auffichlåget/ febr fofoun.

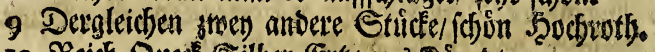

2o Reidf=Sued=Silber Ers/ aus Sirnbten.

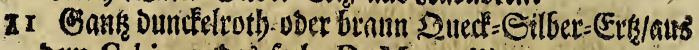
Dem Cabinet Des feel. D. Menzelii, utuntur pro IEthiop. Mineral.

12 Sabin reid) Duret: Silber-Ers/ aus ungarn.

13 Dergleidben felor ichon aus Sft= Smbien.

x 4 Dergleidjen in barten Oeftein aus Lingath.

Is Dergleiden mebr S̈bloreicf.

16 Dergleichen ganis Maffiv.

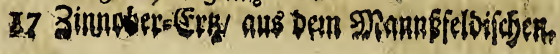




\section{粼 ( 127 ) 綮}

Is Cin Etuid gebiegen ännober/ bon Efemnis/ biel Bovlo balteno.

39. 3innober:-Eris / aus Surot.

20 Edjiefier mit angetwadjerenen Binnober unb angefilos genen Rutuffer.

21 (Ein ander 3innober baltender Edjieferffein/ woburct) Golle=2lbern forsen.

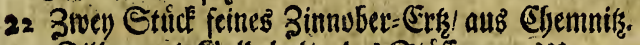

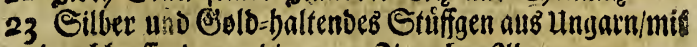
Durchlauffenten geviegenen 3innober=2dbern.

24 2lrtige 3innober=Etuffe/ in Taldetioben Gejeftin / worn Econecberg.

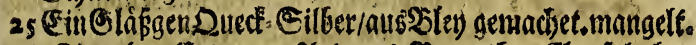

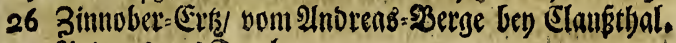

27 Intureres aus Tyrol.

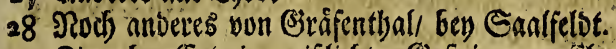

293 innuber:-Eres in weiflichten Geffrein/ yom Elruufthal.

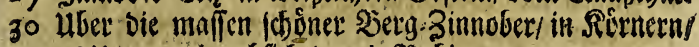

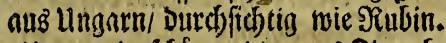

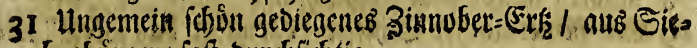
binbuirgen / fant Durchjicitstig.

323334 Einige Labores bon Figitung bes Duecte.

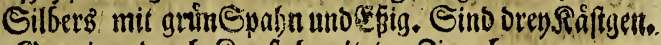
35 Giemeiner Durd Siunft bereiteter Zinnober.

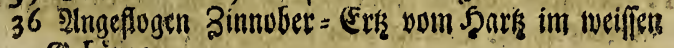
अebuirge.

37 3imnober-Eris im Edjieffir.

\section{Ad(d) \\ Metallifde $=$ Sd) efer}

So eigentlich ju Den Siupfer. Ersten geforten, weil aber einige auch $\hbar$ und D balten/ bieber geferget nootoen/ und theilen fich folche/ in :

(a) Metallifde Sdiefer allein, von al
lerband Alrten. 


\section{结 (128) 翡}

( $\beta$ ) Metallifde Sdjefer, fo alfertsand Sivauter=Figuren præfentiren.

(y) SRetallifide Sifiefer, to allerband Fifhe: (Geftalt prefentiren.

VIII. [a ] rretallifthe Schiefer alleinI von allerband 2 retben.

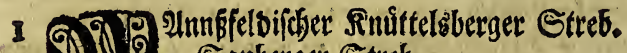

91. 92 Enuberget Streb.

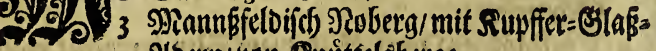
21bern/ von Sinittelsberge.

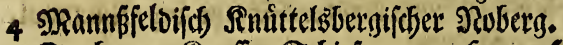

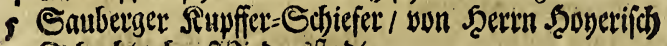
Schadte ben sigiederergftadt.

6 ziegen uno Sauberger Etreb.

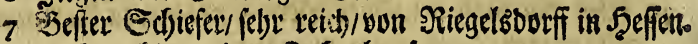

8 शobers Ginter bem zectenbaufe.

9 Ein SMineralif Ger Sdjeffer/ barimen bas Metall wie Sdjott lientst.

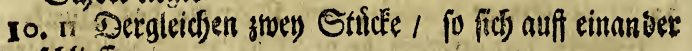
ifflieffer.

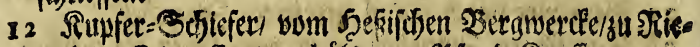

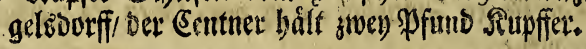

13 Seeetbaulier/ weldjer als mit Edjrot befofufien / auşa fielet.

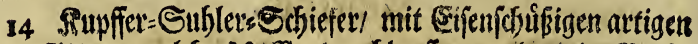
Bittern/weldse Maffiv-Durchlauffen/ und eninige:3uct)= ftaben/ als A. H.F. E. prafentiren.

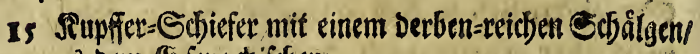
ous Dem Eiventadifideren

I6 Ruppfert=-Sublet:-Swjiffer mit Siefigten gelben Can。 cellis. 


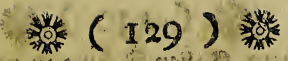

\section{[B] Mretallifhe Gdblefer / to allerbano}

Lirånter-Figuren prefentiren.

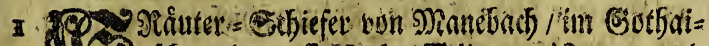

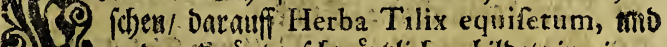

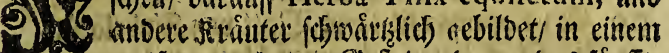

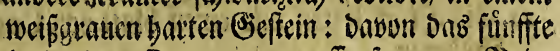
Paquet, ber andern Ravageson nuffigefangenen sbric= fen banbelt.

2 (Fin anderes/ welther and) aufif farten grauen Ges

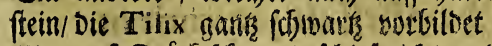

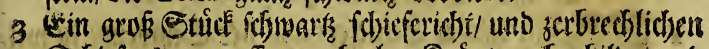

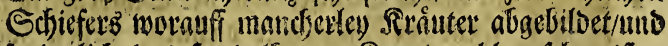

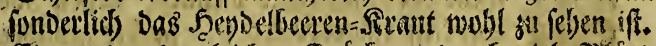

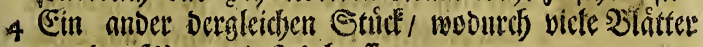
yon Der filice paliaftri lauffert.

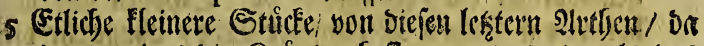

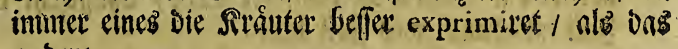
andere.

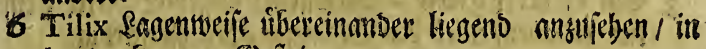
baiten Brounen Geffein.

7 Dergleifien mit Polypodio und Iride palufri vet mildjet.

8 3men Etatid Tilix, wie bie sorige.

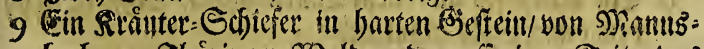

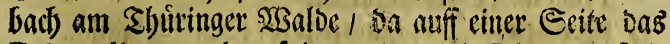
Polypodium, unb ouf Der anbern Die Iris paluftris fauber zul fellen.

o Ruvfferfubler Schiefer/ fo von einer weiffen Materie einen Dentritem prefentiret.

II Siriuter Sedjieffer mit fiern leber Rraut/ Echilff ano

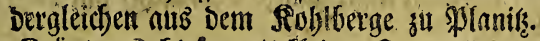

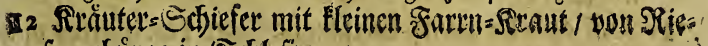
fenthebuirge in Sodjlefien.

I3 Dito yon Gablan.

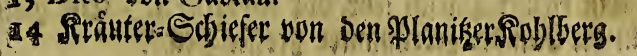

$$
\text { s }
$$




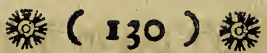

15 Dito mit bem Saf̧en Sdjwank von eben babet: 16 Dito.

17 Dito yon Riejengebuitge mit bem Rebet=Sirnut.

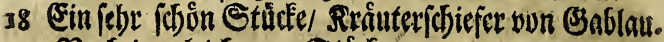

19 Nocb Dergleidsen 2. Otuite.

20 Ein Tophus mit einen Slatt von Sinoelorice.

21 Ein Stric letten mit einen blatt von Commodau in soibmen.

[r] Metallifbe Schiefer / fo allerbans Sifhe: Geftalt prefentiren.

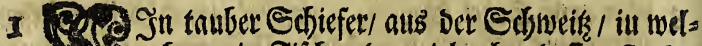

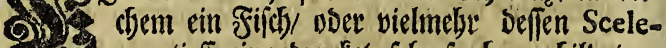
2. 2 ton tieff cingebrudetet fejse fauber gebilbet.

2 Ein Supffer s reeidber Sdjieffer / aus Slyiritu getw/morauff ein Pafferculus ober Ectjollen jebr fau=

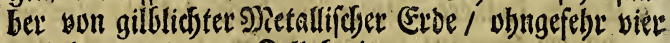
Zull lang/ tund stwen Boll breit.

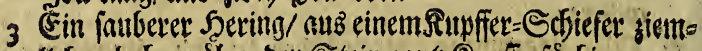
lic) erboben/ úber Den Stein ganf̧, Nupfierfárbig.

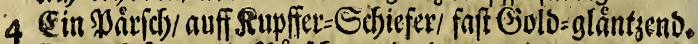
s (Ein geftaimmter \$árí) / wie ber vorige/ weniger glíntzend.

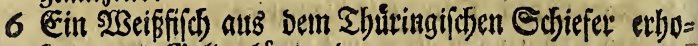
ben/gants Gn lb=glåntsenb.

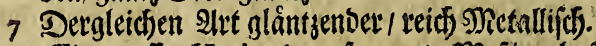

8 Eiin groffer Haringites, fo gants \$Nefing-bafftig aubs fiteftet.

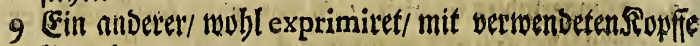
liegent.

10 (Eine/ wiemobl nicht gantge Corauldel gat exprimitet.

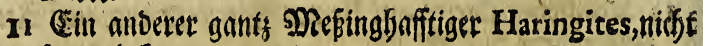
ponberlicf) exprimiret.

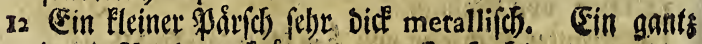
in bie siunde gefrummier geofer sededt. 


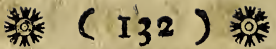

2526 Eit balber Rarpfen / zienllids gró uno felse me-

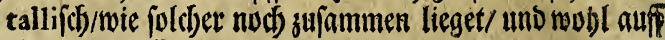
einander paffet.

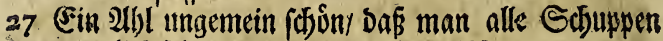
cigentlic) jehen fan/wiegt wohl 8. Pfund.

28 SToch sine Sarbe obne Siopfi.

29 Ein (c)biner exprimirter Fiid) mit einem búden sopff.

S.: Ser Srften atbrilung.

Non Der

Sinfften Dronung.

Drifter Unterfated

bealt in fich

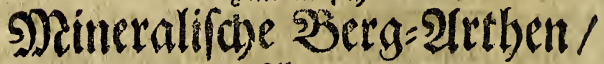

Alts:

Sobotte, Antimonial-Erke, Artenic-

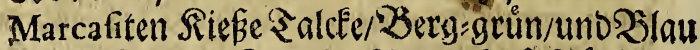

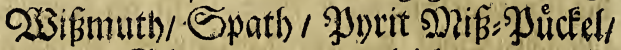

Slende und Dergleichen. श्Sic audf)!

Sineralifde Dinge fo aus ben Sirbeiten erwachien.

21B:

Gold und Gifber = (Blatten, aflerband

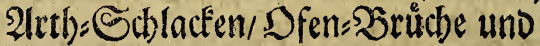
Dergleidien.

zie 6. Sdbublabe.

Unvoflesmmene Mineralien.

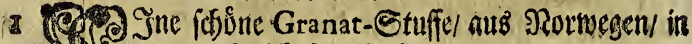
Q)

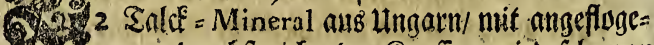

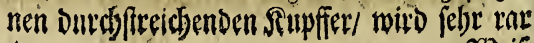
gendidet.

3 भुsei 


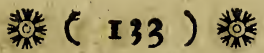

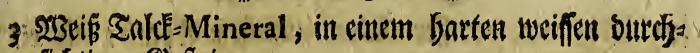
fidjtigen Sieftein.

4 (Eine Stuffe Granaten in Salde getwadjien.

5 Dundelbraun Salde=Mineral feljt bart wom Etcite.

6 Sulofarbig Saluf $=$ Mineral.

7 Sraun butd)fid)tig Marien-(Fiß auts \$reufen.

8 Deffelben Matrix in barten Bseftein.

9 Talek=Mineral in weiffem Beftein.

1o Talde-Stuffen/ Darinnen unteiffe Eifenfarbige Granaten ftecken.

Ir Eine artige/ als Amiant viber ein ander getwadjent sald Stuffe.

12 (3ritnlid)t Sald Mineral, nus Stolien mit Girnuaten.

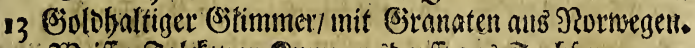

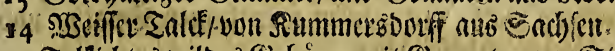

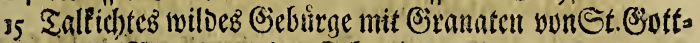
batots Derge/ ans Det Ed fuch

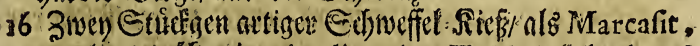
bon lanter tofereinander liegenoen Tubis leftebend/ans

Dèlli Şartge.

17 Ein ran Ettidk Marcafit, barinnen ein Sndifere En-

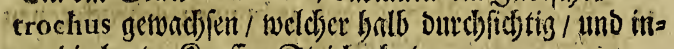
wendig lnuter Supffer=Striche bat.

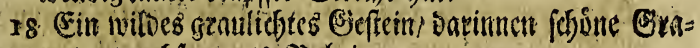
unten wad)fette auts Wobleimen.

19. 23 ignutl) bon Sours.

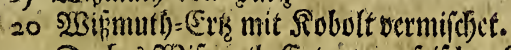

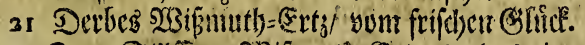

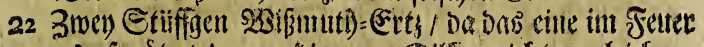
nutgecprutzet bem geóbienenen Eilfer nidft ungletíf.

23 3wen. Derbe 223 ismutl) Etuffen/ non Ecfineeberg.

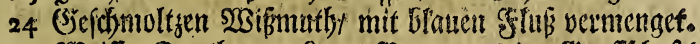

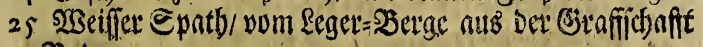
Saben.

26 Durchfichtiger Dutarts/ wie ein Entoirter Zutlerften=

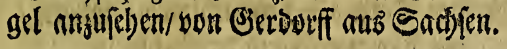

$$
\$ 3
$$




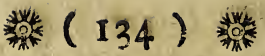

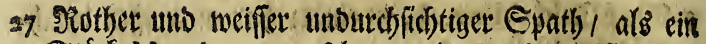
Ettúck Marcipan anjulueben/ mit etwas anigeflugenen Rupfiet=-Ries yon Girener Fumb=-Sirube ben Stryberty.

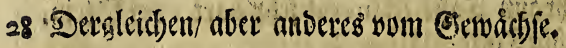

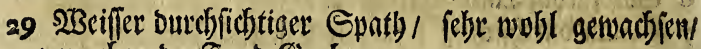
von elen der Syund=-Grube.

30 Deralkidsen/ aber Hleiner yom Eetwódbfe.

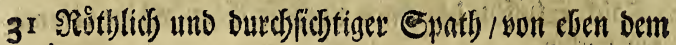
Drte.

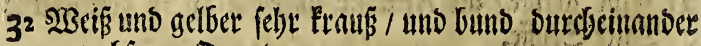
gensadjener Duntts.

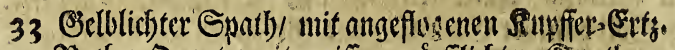

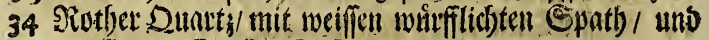

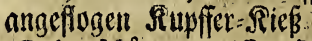

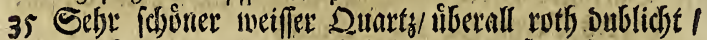

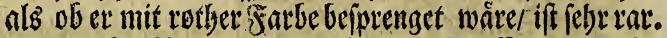
${ }^{6} 6$ (Ein reidfer \$orit/, mit angeflugenen Maffliv-Marcafit。

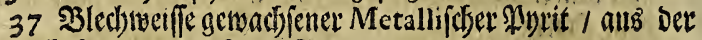
Edjweit yum Reger-23erge.

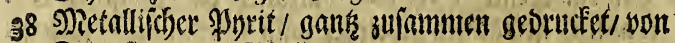
Stcinfsadt/am ఇibein.

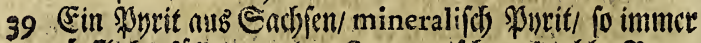

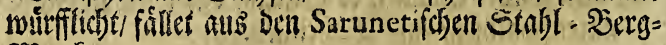
șercéen.

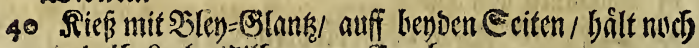
ein ball Rott) eilber/ bon frenberg.

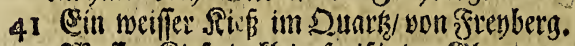

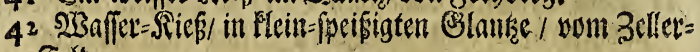
Felbe.

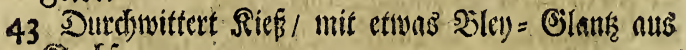
Endjicr.

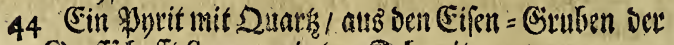
Graffichaffit Sarunet in Der Socfiveitiz.

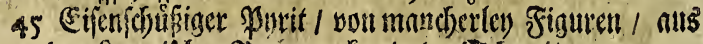

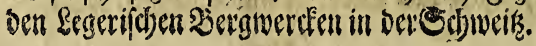




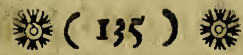 \\ Die 7te Edfutblabe.}

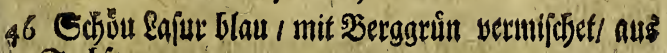

Eachien.

47 Dergleidjen Silberthaltig/ wom Saarke.

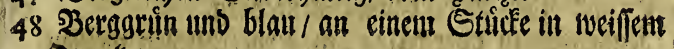
Duark.

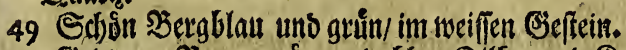

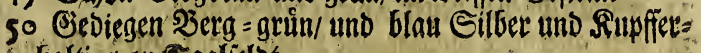
baltig/ioon Eaalfelot.

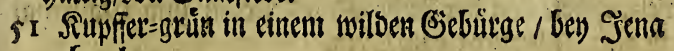
gebrodecen.

\$2 Silleerfarbiger Metalliicher Edjiefer.

53 Ein artig Stuffigen weiffer Spath / mit Siller: = Evis vermichset.

54 Eine Derbe Blenbe bon Elautftlal.

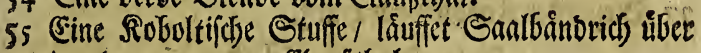
einanber treeg/ yom Elaustfal.

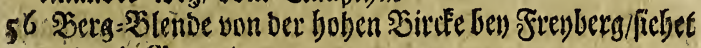
nü̈ wic Siranaten.

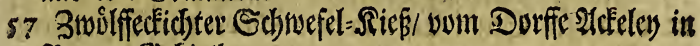
berner:-Bebiett)e.

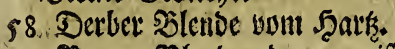

59 Straume 3 lenoc uber unreiffer OSlanţ.

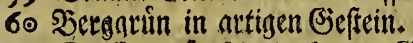

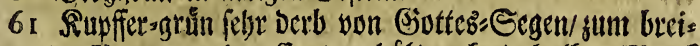

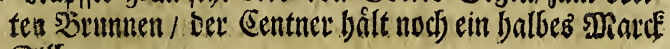
Eilber.

62. Ein ficter Mineral, fo mir vor Materia prima ge= fojictet nuveren.

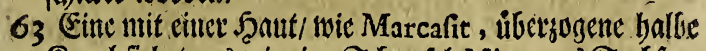

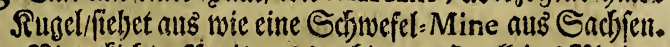
64 Sierectictiter \$yyrit/aus Der bintern Dutell Des Sibcins.

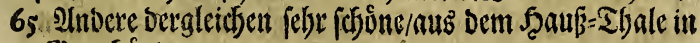
Graubindeen.

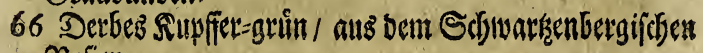
ßefier.

$$
34
$$




\section{粼 $(136)$ 獎}

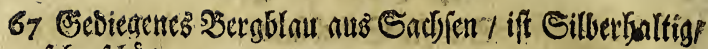
feffr idobit.

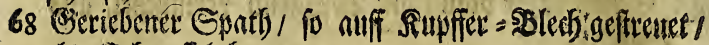
als ext)weffal brennet.

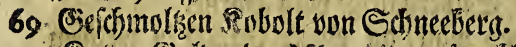

go Jiakgen=Siolo voer Mica, it zerbrecblichen weiffen

Beffitin.

7L Enalfelbicher befter Robolt mit Blütlse.

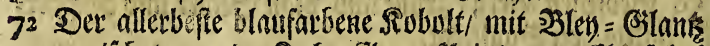

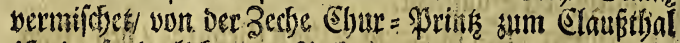

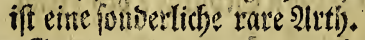

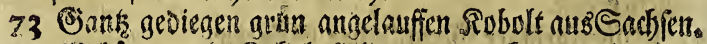

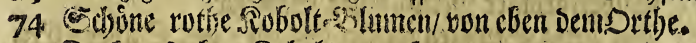

75 Derber farben Nobult mont Scart.

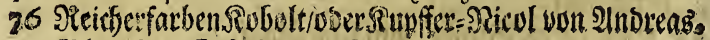

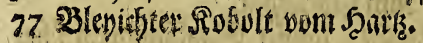

$$
\text { Trie } 8 . \text { Schublade. }
$$

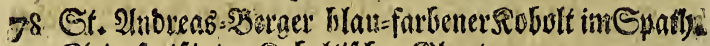

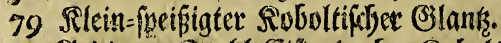

\$o Gebiegen Stnjl: Eifen Derbar fubolt vonedjneeberg:

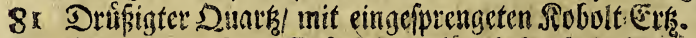

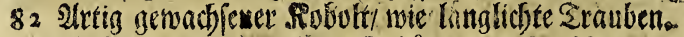

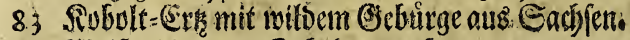

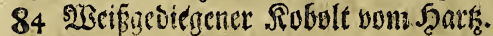

85 Récolitiô Mineral von 23 ernigerwoe.

86 Eifenfolifisiger Robolt / aus Der Seim = Grube vor

bertin.

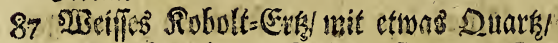

s8 Sicfter Froblt/ bun Jubnan Eeornen Stabt.

89 Eir Stut A ntimoniun, ats Lingntm/Baldbaltend

* nit Wintutatothen Blumgen.

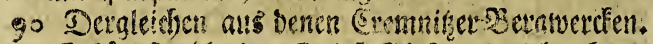

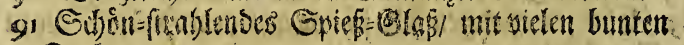

Saruell.

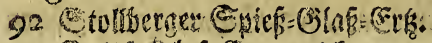

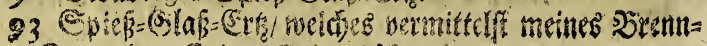

Epiegels nuff einer Esiff geformuligen. 


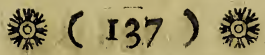

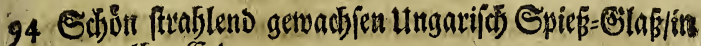 ciner gelben Erbe.}

95 Dergleidsen mefre neiflictst.

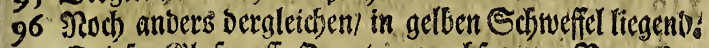

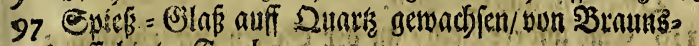

Dorfif binter fretsberg.

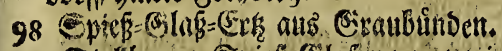

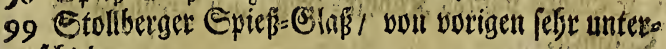

ichieben

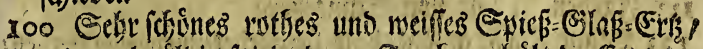

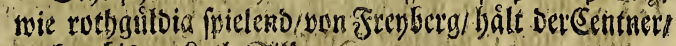
nutb) 3. bis 4 . Ruth Silber.

Io1 Lumgarifoces gattes Antimonial-Erts.

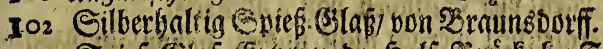

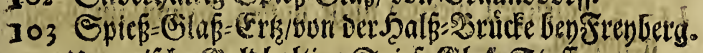
104 Ungarifote Sulbbaltige Spief= EIInb=Stuffe.

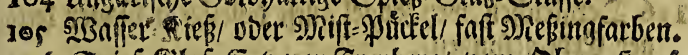

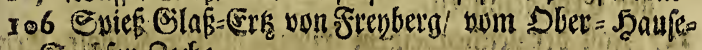

\section{Sadijen 3eche.}

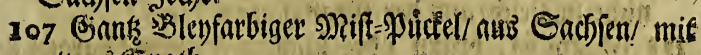
etruas epath.

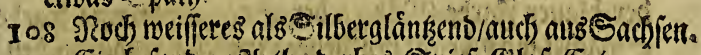

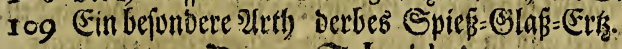

$$
\text { zore 9. Schublabe }
$$

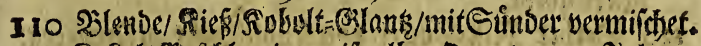

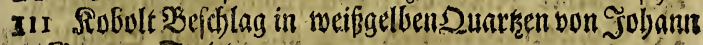
Georgen Etrot.

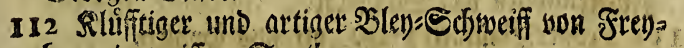
berg/ in weiffen Spath.

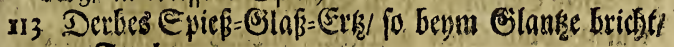
von Frenberg.

114 S3affer Ries in grauem Bebuirge.

IIs Speanter doce 2̧ince.

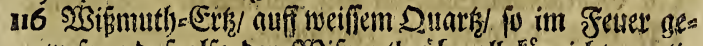

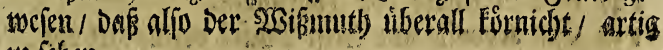
sul êlien.

$$
\text { os } 5
$$




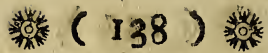

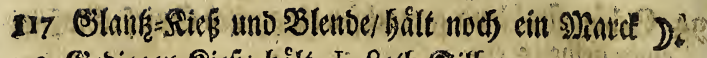
18 Gediegen Rieß! bålt $\frac{I}{2}$ Rotl) Eitber.

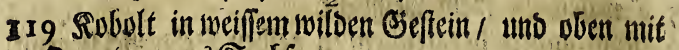
Duarken/ aus Sadjent.

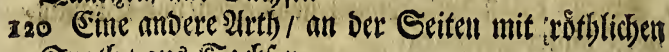
Spatly / aus Sadfien.

22r Edjines buntes Robolt = En/ / bey Jtenberg in Eadjicn.

222 Jarben Sobolt won Ectineedberg.

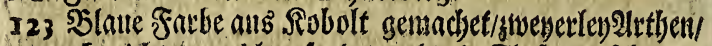

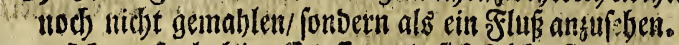
I24 Marcafit baltige Stuffe / wie fich folche finver su Nezingen/ vier Stumben von Tibingen.

725. Dergleidfar werben nicht unweit Dabon/ im 23 affer gefuntsen.

I26 Deroleiden jwen Singel aud) Dajelbft in blauer Lettiger Eetbe gefunden.

127 Frnft bergleidjen/ einent Eifenfdrifigigen sobolt = $C_{r}$ nicht umgleids/aus Der (straffidsaffit Sulms.

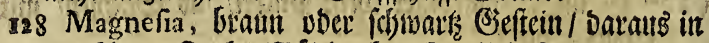

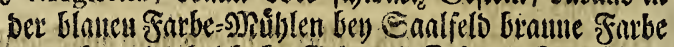

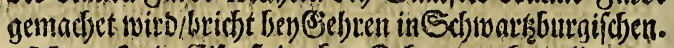

129 Marcafit in (Eijenffeciu/ bey Sdjwarkenberg lieget Nierenmeife.

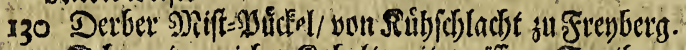

I31 Edjwarker teidjer fiofolt/ mit weiffem Spatf/ yol Surufrenftollen bey Saalfelot.

I32 \$iblet Giaflimei) yon Zgott nus \$oflen.

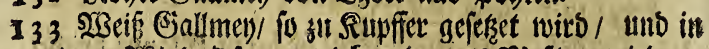
einem 23 ind :Dfen getrieben/Daraus Miéfing wirb.

134 Erriner Garllmey.

135 Etvilfergifber Bntlimen gant gelbe.

136 Gebrannter Batlmey von Sornemifzo

I 37 (Sratter. Robult won \&ubenftein.

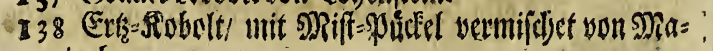
rienberg. 


\section{整 (}

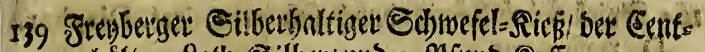
ner bâlt ". Soth Eilber/ tmo 9. Yfind Suffer. 140 Eime Befondere Altrfy yen unteifien Electro piretet th alle Sarben.

341 Rovbult mit \$Si

\section{Die 1o Ethublabe.}

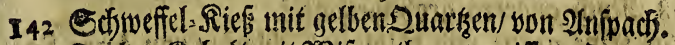

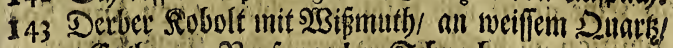
won Eartfortur ?Reufang/ bey Edjnebberg. 144 Edjneeberger Edjwerfel=Riés.

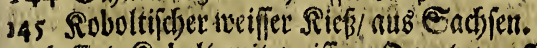
I46 Encr-Sobolt mit weiffem Duark/von Magenkits.

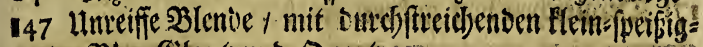

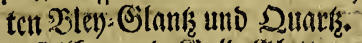

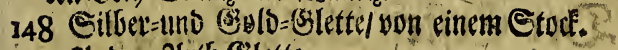

149 Iinvere Sititl) Blette.

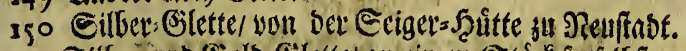

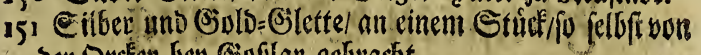
Der: Drteler bey Goufiar gebradot.

of 2 Blen=-Gilette youn Reuffabt.

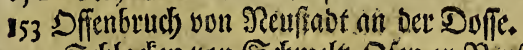

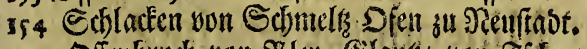

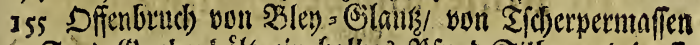

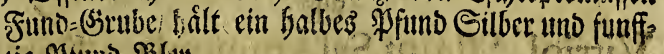

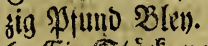

s 6 Ein Etuded von ciner Mineraliffeen Mixtur, von eftlithen \$funsen / Derglecisen cine groffe Menge at Stargaro in \$ummern esgraben werden.

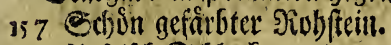
158 Infritid) Sd lacte.

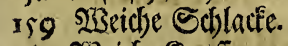

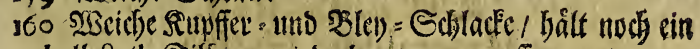
ball \&otl) Siller / wirb aber wesgenorffen.

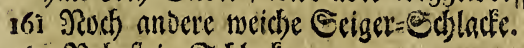

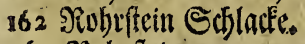
163 sivblyftein, 


\section{势能 (140) 耀}

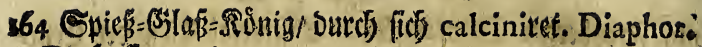
D. Jcoffutaung.

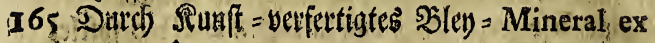
万) uno 金.

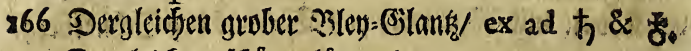
367 Dergleidjen ichơn glántsent. 368 Neds anderes grob=ipeipigtes.

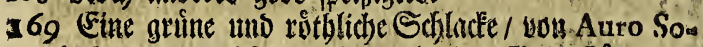

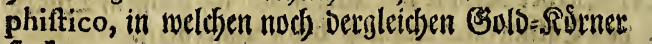
frecten.

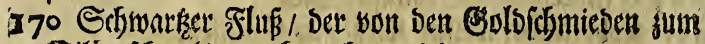

Eilberfojmelizen gebrautset wirb. 17 Dergleichen von anberer Farbe.

\section{Der Erften abtbeilung/} 23on der zunftent Orotunt
Sierder unterforior scalt in fich

\section{Mineralinto Saltsel}

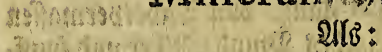

Vitriol, $\mathfrak{A l a u n , S a l A m m o n i a c . , ~ B o r r a s ~}$ und andere Salfsel

sssic autic: :

Mineralifue Sdinefel=:2ten Rieffe und SteinsRoblen. Der 4. Kaften. Mineralifose Salke.

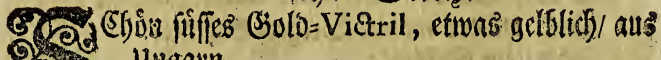
802. Ulignin.

2 Dergleichen ing gruine fallens. 


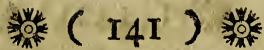

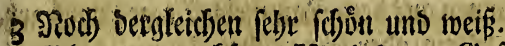

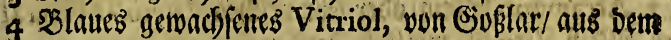
शRammelsberge.

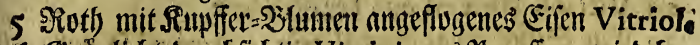

6 Gutunlicht burchfichtig Vitriol, aus \$reuffen/ wirb bers Dem SEernfein gefunoen/ uad von D. Scartmannen Vitriolum vitriforme genannt.

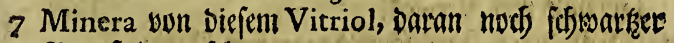
3ernftein

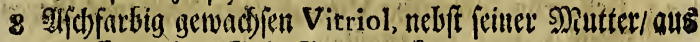
Den Ercmnif̧er Ejuld=25ergmert'sn.

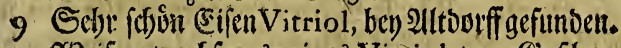

so $\mathfrak{X}_{3}$ eip=gemadjenes zeines Vitriol, yon Goplat.

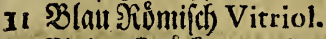

12 Eiaine Etidfe von einem fojonen weiffen Vitriol, wel-

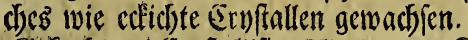

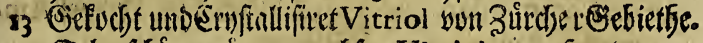
${ }_{4}$ Eelje fojôn gruin getwadjen Vitriol vom scatr.

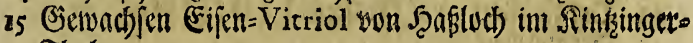
Thal.

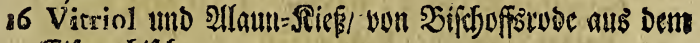
Eifenncbilchen.

I7 Euprifcher Vitriol, io fojun bodblat aus ber Braffe

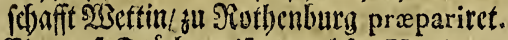

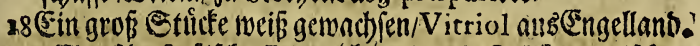

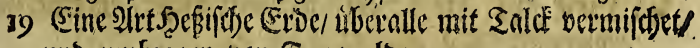
und umbsogen son Freyiwalde.

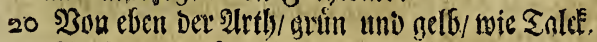

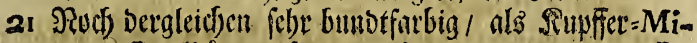

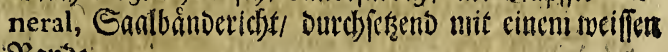
Siantive.

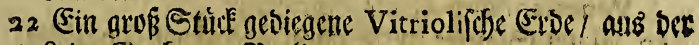

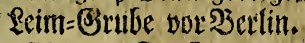

23 Vitriol-Stuffel auB Den Strweiractifonen 2lpen. 24 शieide Vitriol-Stuffe yom Scark.

25 Eine andere 2lith/ in ciner barten braunen Eroe. 


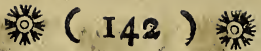

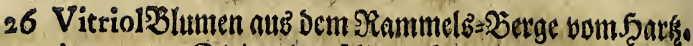

27 Atrament-Stein/ voer felbr reidjes Vitrol-Mineral.

28 2Bei Vitriol, mie $\mho_{i \beta}=$ Zupfen.

29. Vitriol-Mineral aus Der Scfjuctôs.

30 Ein Stufk Englijhbes Vitriol - Mineral fo nur i mit aus frugen im Rodfen zu gute gemadset wiro.

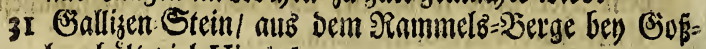
lat: hâlt viel Vitriol.

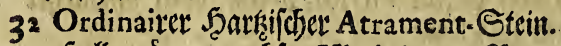

33 Jetlegrín getwad)fen Vitriol, vom aimmmels=3berge.

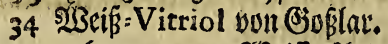

35. 36. 37. 38. 4 25seiffe Anten gewarbjenen Vitriols in

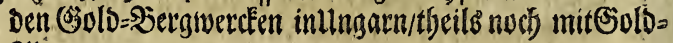
2lbirn vermentet.

39. Selbft getwadjen Salpeter won St. Mivriş in ber Edjucitif.

40 Sal ammoniac, nus Africa, teben bem Sempel Am. monis, Anno ${ }^{2}$ 00. colligitet.

4 I Dergleichen aus Štalien.

42 Dergleichen gelo.

43 Gegraben Sals aus \$oblen.

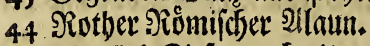

45. Vitriol-Się von breiten Srunnen bey Sreyberg.

\section{Dex 5. Zâfen.}

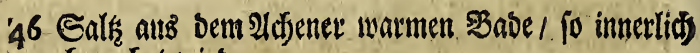
gebrauchet wits.

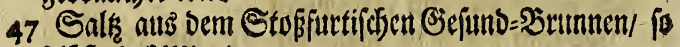
felbft deftillitet

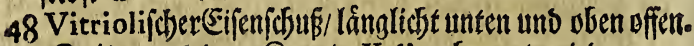
49 Salk! aus Dem Sraute Kali gebraunt/ wirb sum Venetianifosen Gilafe gebraudjet.

so 2llaun Scfiefer mit Sajwefel=-się yon Saalfeldt.

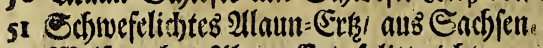

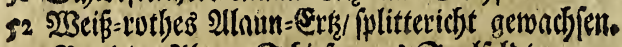

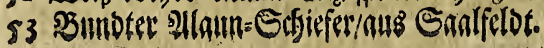




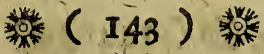

54 Gin Stiud geflofien Salf/, broun gemauturlt/ wie gellifen/ und Etcinflippent.

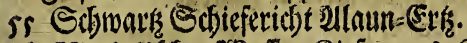

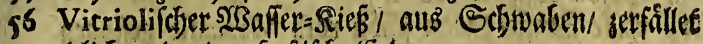

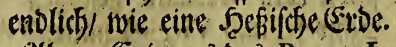

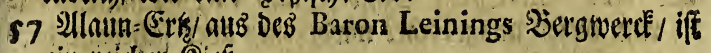
ein reidjer Rieß.

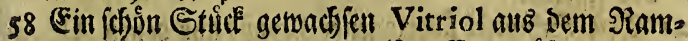

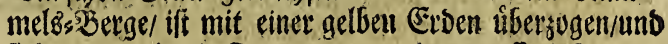
fiefet aub/ nls cin Dommen von ciner groffen MPaung Şand.

\section{Sd)rofel=stie.}

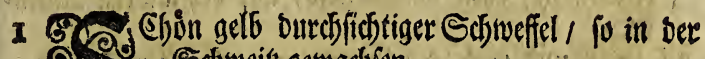
or. Schweify gemadjien.

2 Durrdffichtiger lebenoiger Surngere-edinefell wiro bey Bactiac im soerluer-(Stebiet)e ges graben.

3 Sdjun balb Ditreffichtig gewadjener Sdjwefel vom Pammels=\$brge.

4 Rebenbiger Sdjwefel aus Candien.

5 Ejervachjener Ectwefel yon Solza terra ben Neapolis, olnneit bes Vefuvii.

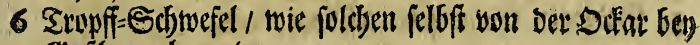
Giveslar gelanget.

7 Sd)un unformlid getwandener Edjwefel.

8 Sdjuner Sdjwefel in Etâbaen.

9. Deraleidjen/ was istedjter und dinnet.

1o Dergleichen/was Differ.

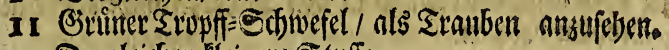

12 Dergleidfen fleinere Etuffe.

13 fingelformiget Sfinefel/ aurs Scandien.

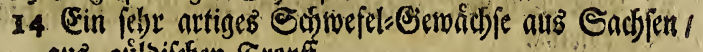
auts gúlbifofen Stopft.

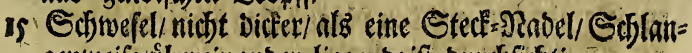
genweiferbereinanoer liegend/ iff ourdfictstig. 


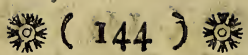

I6 57aw Mineral mit angeflogenen Edjwefel.

i7 Eschefefl=Stuffe/ biel Marcafit balteno.

18 Echon ruth Marcatit.

io Gine Stuffel batan idfón roth Marcafit oefloget.

so Atrament-Etein/ Sdjwefel und Vitriol baltend/oa

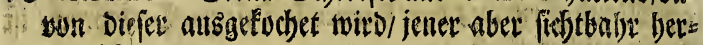
vor idjeinet.

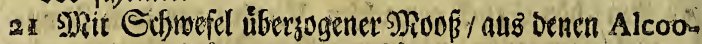

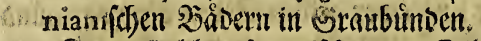

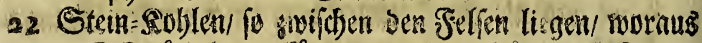

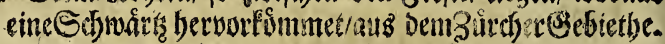

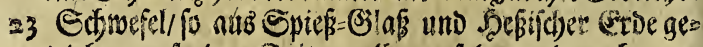
trieben/auf einer Eeiten gelb/auf Der ambern bram weth.

24 Gelb und roth Auripigmenc, tiefjet noch lebs Sdinefeclidyst.

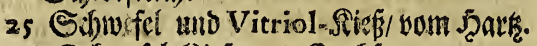

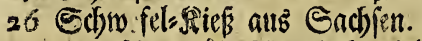

a7 Sseirifder guilbicher Sdjmefel und Vitriol-Piefo.

28 Sdbuefels Siep.

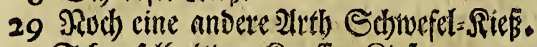

30 Sdfwefelbaltiger Rupfert= Sieß.

31 Edjin gefárbete Sdjweple=Drufe.

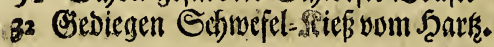

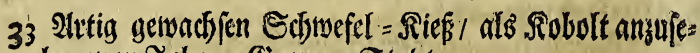
ben/ von Jobann Siveraen Strút.

34 Selfr bunte Stein=Poblen yon Frenberg.

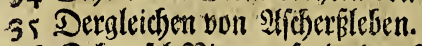

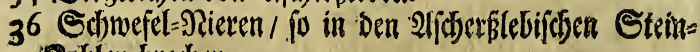
sobjlen bredsen.

37 Engliphe Etein= Roblen.

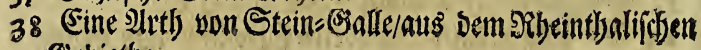
Gebietle.

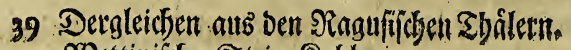

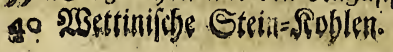




\section{紫 ( 145 )}

41 Slanif̧er Etein= Seblen ben amidfau.

42 ??ach cine Antl) Englifther Etrin=soblen.

43 Sdjwefel = Mineta yon Reipusig / befefet auls cinem

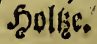

\section{Der Erften 2 lbtbeilung}

\section{Ton ber}

\section{Jưnften Drbmung|}

Junifter Unteriforio

f̧ấl in fị)

Allerband Quarket, Druren, und aller.

band Arten von Ernfrall = Stuffen

$$
\text { und }
$$

Imetbiften/Snacintber/Srana= ten, Eorallen, Topas/ und Derglei: deen borfterlend.

Der 20. XAaften.

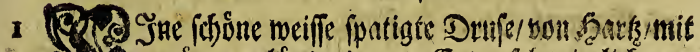

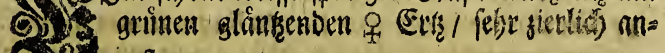
2.23 geflugen.

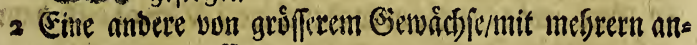
geflogenen of Eirge.

3 Gine andorel wie Seers und Thal gewadfen/ mit mentig

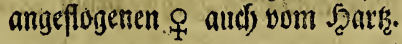

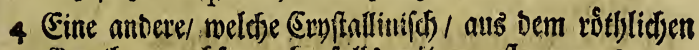
Spath) gemodifen / ebenfalls mit angeflogenen o

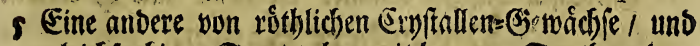
gleidj/arbigen Epantl/oben mit bramen Spatb/ und o

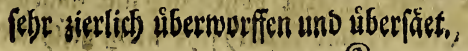




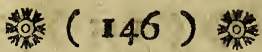

6 Cine andere/ bicfer nicht viel ungleich/ofjne Daß biernuff

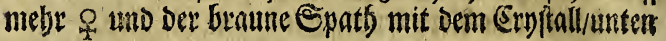

vat ino veren verniifdet.

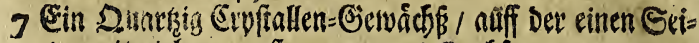
ten mit vielen angefígen o oub Eachjern.

8 Ein Cstide beffeteni nus Inuter Tubulis umferer Cadmia factitia voer Tutia nidft viel ungleidf.

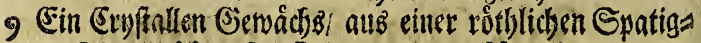
ten Metallifojen e tuffe bermus getwachien.

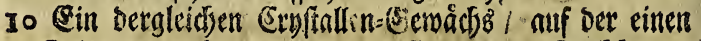
Scite mit vielem Metall angefiogen/ mus Sachjent.

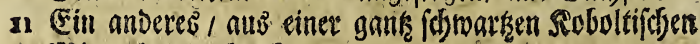
Miner berwor bredsens.

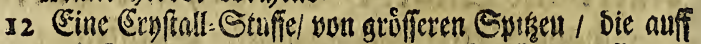
einer Eeitert/mit weiffem E patt) und Supferer angeflogen.

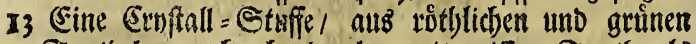
Spat's hersor=brechend/ oben mit weiffem Spath/als Salls berworfien.

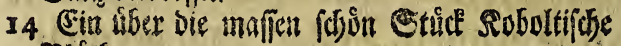
slititfe.

15 Eine rothe Eryftall-Stufiel aus einem 4 Stein Jere

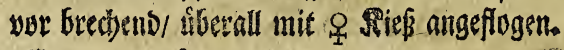

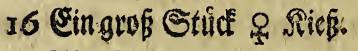

17 SilexSanguinolentus, wowon Becmannus in fuaDif-

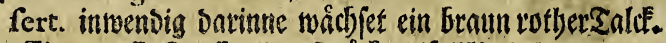

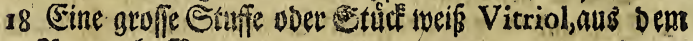
शimmmelb=5erge.

x9. Cine Maffa yon Stetin-Erbel Topho und petrificir-

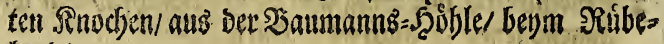
landt.

20 Eir Sergleitsen mit Topho iberzogen.

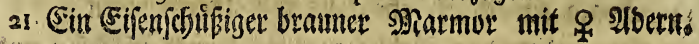

$$
\text { Der andere Zaften. }
$$

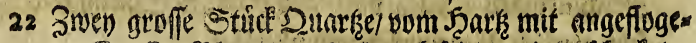

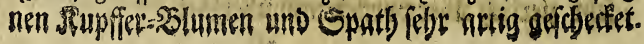




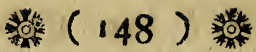

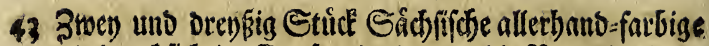
und Durdjfidtige Drujen Da immer Die ?atur in cinem anbers fpielet/ was Farbot und Beffalt betrifft.

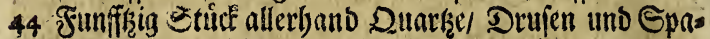
then won unterfobiebenen Jarben/uno Seffalt/ alle nus Eachiern.

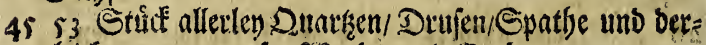
gleiçen wun curiöfen Sißudb) itno farben.

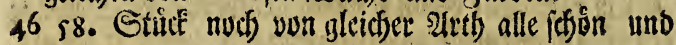
curiös.

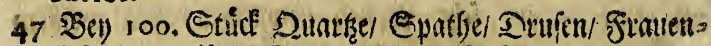

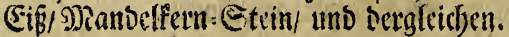

48 Ein Scaften mit felyz vielen Steinen fo eine gerviffe Figur vortiften/Fenerfteine mit Eurallen. Stem 2 Bie= Ietlen Eteine und Sachen won weldfen man nidst ge= wis recmutit wa fie berjern ober was es iff / auds die fonft tibrig blizber.

\section{Fimftret Dromung Burete 2lotiocilung \\ Şâlt in fich) \\ Zon Mineralindyen Sieidee, \\ Erfilid) 1 \\ Dit srocn/}

So mohl gefiegelte/ als ungefiegelte/ bie in ber Medicin und funften im semeinen $2 \mathbb{B} c$ fen gevráudblict)! wie ands)

âventens

\section{D) Gre}

So wofil Ede Steme auzo Orient und Occi-

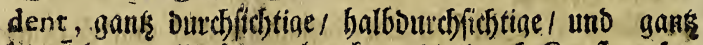

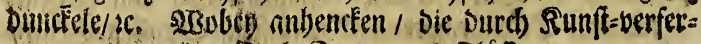
tigte Evele Steine/ uno Stlúfer als auds: 


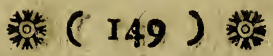 \\ (1)}

So megen ibrer allerhand befondern (3) eftalten. aufgeboben werben/ anbey allertjand anbere Steite/ bon Marmor, \$pyriten/A mianten/Spedfffeine und Dergleichen.

\section{(Endlic) Drittenz: \\ Berfemerte Solnge।}

\section{Eo mobl}

Derfernerte Muldiln und Sofneden, als audi andere verffeinerte Sad)en/ als Sinos dben/ Sruedte/ Şolk/ uno fo weiter. Der stwenten 2 abtbeilung

\section{Bon bet}

fơnfften Oronung

Erfter linterfideto

5ृålt in (iid)

\section{Die Erioen/}

Whelde fonohl in Der Medicin, als ronft in gemeinen S:efen geftatchet werven und jwar erfflic):

\section{Sie ungeficgite,}

șeldsem beynefuget cinige

$$
\text { sizas=isteme. }
$$

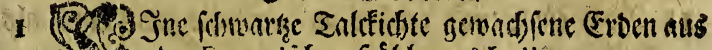

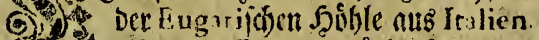

6.2. Eme neiffe etwas tithliche Materie, balte es na: pine Althl / Stein = Gialden / ift Anno 169. an D. Scetmannen/ atb Dff = Inbien Bcidicfet troitien.

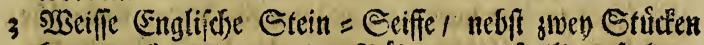
brauner \&attem/ aus ben Bábern icr f̧eiligen f̧elena/ in Italien/fio fratd ausgcorudfnet. 


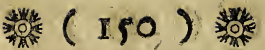

4 Detaleiden yon anberer 2lteth / aus bem Drfe / foll eir gutes erweidfendes Stücte fenth.

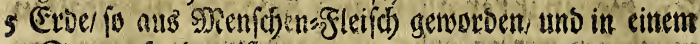
Saris gefunten ift

6 .

7 Erve aus ber Grotte Des Sceiligen Pauli,nus Der Snfut Malrha, Darinen sin foum einer ballen finfen geoffer

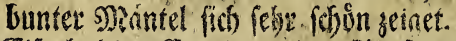

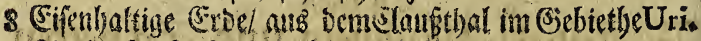

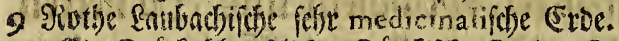

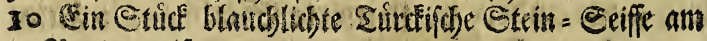
Sande weí

II Ein Ettice Sinnober rother Bolus.

I2 Crmulidgt Etciunarde/ fo zwichen Eanoftein gefunben wirb/ in ber Sdineth/bes Steinfrabt.

33. Finc betl: braune lichte Mediciniffje Evoe.

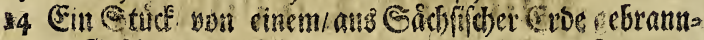

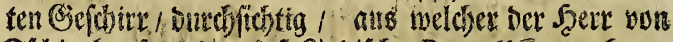

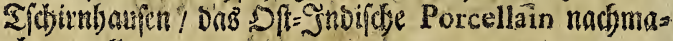
d)en wollen.

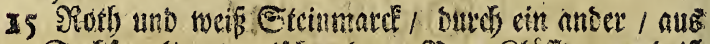

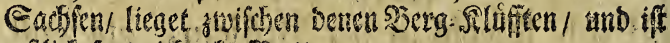
exflich) fo (beich als sbutter.

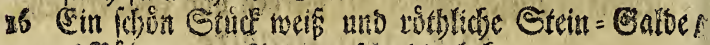

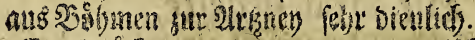

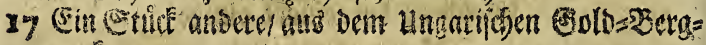
worte.

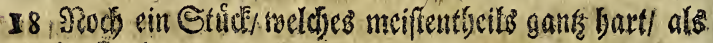
cin Etrin.

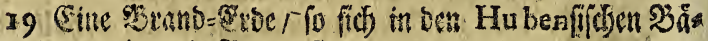
bern/ ohnmeit 53 aben finibet.

20 Eelie fobint Armenifoer Bolus.

21 (Ein rutiblicher nod) gants rofer Bolus.

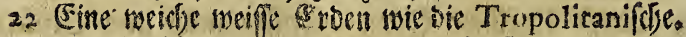

23 Ein Sovensontiger Bolus, yon feyftabt aus Det ossetternat. 


\section{(I5I) 粼}

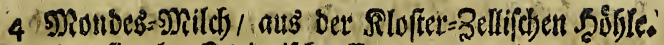

25 Ungefiegelte Etriegildbe Erve.

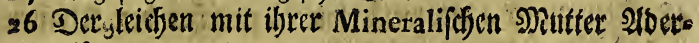
weilfe vermenget.

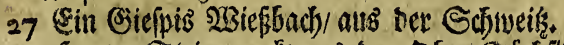

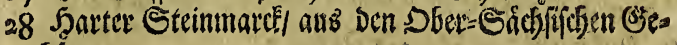
bưrgen.

29 Sine weiflichte Groe von Eceflyolshbeim/ ben Etrapj? burg/ mit vielen fartien gefdecteet.

30 Eine braune von eben Den Dute mir vielen Farber marmoritet.

3r Eire anbere neiflicbte.

32 Dergleidjen?

33 ' Socts ethe nubere different.

34 Dergleidfen in etwas unter[dictert.

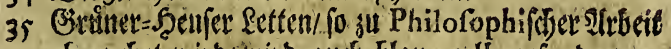

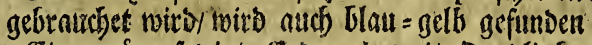

36 Esine griue fettigte (Eroe/ Dbmtreit Duenlinfurtg ges funien.

37 Cine rótblict)e (Eroe/ mit blau uno antern fanben I artio marmoritet.

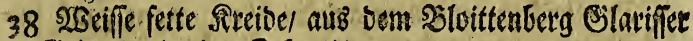
Gebieths in Der Erlyweits.

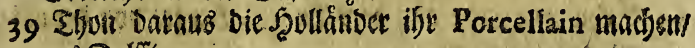
aus Delfft.

40 Dergleidsen won einem ambern Sorte.

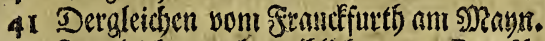

42 Deraleichen mefor getbäicht/ uon StrabGurg.

43 Gelbe- Mineraliffec Siegel Erbe auts sobmentwelche wenn Beffoitr baraus gebrannt wird/gank toth wirb.

44 Eerie/ bataus in China Das féinte Porcellain ge= Erantht wirs.

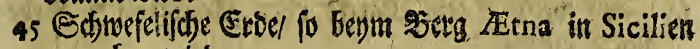
gearaben soiro.

46 Dergleidenen etruas beller.

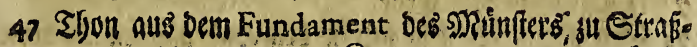
$\Re 4$, burgl 


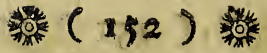

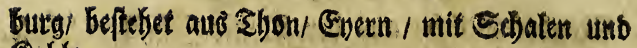
Sioblen

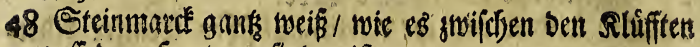
auff Dem Şarts Ju finven ift.

49 Eroe ober vielmeils cine IIth von Babeftein / aus Dem Earla=? Bab

so 2lufnutf oes şerges Vefuvii.

gr Edjwifelicbte Eroe / obnweit bemfelbèn gefunben.

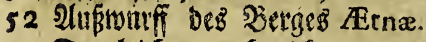

53 Dergleidsen nod) nicht ganfr ausgebrannte/unt babet Belb und réthlid anzufeben.

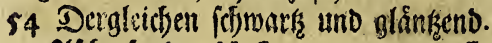

ss 2 igche fo Der Vefuvins ausBgerworffen.

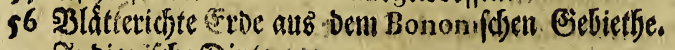

57 Indianilfore Dinte

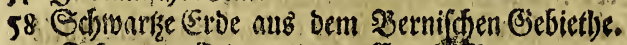

59 Sdjwarize (Ethe aus Dem Engeto-berge.

60 Vitriolifidse (sibe.

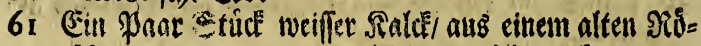

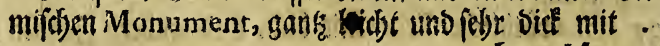

62 Deraleidjen nu einem andern Monument gants uth terfdbieben yon unferm feutizen. Taldé.

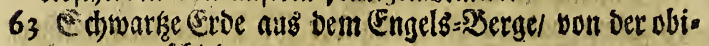
gen unterichieben.

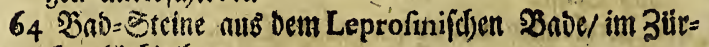
dyer (sisebietfye.

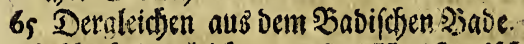

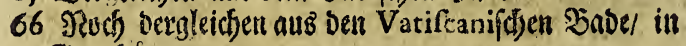
Gratbin en

67 Dernleidsan yon eben bem Drte/ bad) was different.

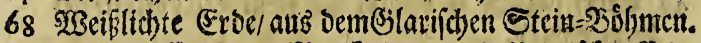

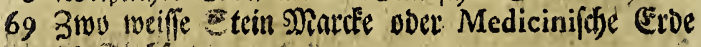
a

70 Sergleidsen ritblids.

7) Tripolitan fóde Eroe.

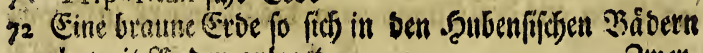
obntweit SSaden anleget. 


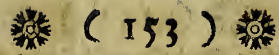 3norntens, \\ Die Sefiegelte}

fo nur in ber Medicin gebrauchet werben/afs: 13 men Stuid Malthefifoge Siegel-Erbe alf einer Seite mit bem BilbePetri/ auf oer andern mit bem Droenss Ereuke.

2 Eine fleine florentiniffe getblid)t/ mit bem 2Bappep von floreng.

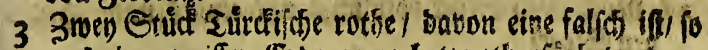
aus einer weiffen Eroe gemadet/ rotbzefárber uno gee fiegelts if.

4 3too andere fieller won farbe mit einem andern Sangel.

5 Eine anbere braune/ movan bie Edr the ausgegangeal mie einem 23 appen/ io aus einem balber 2ubler/urto eis nem Felbe / mit 3. Subiffers:baitfen beffefet / mit Der Beyf(drifft Terra figillata M. I613.

6 Z3:0 andere Surectifobl yon anderer Frarbe und Eiegel als bie botige/ fino sufficibtig.

7 Eine rare weiff? Sizgel = Erber barauff Ebrifti Billo!

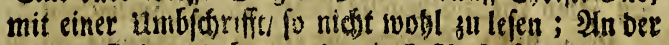
anbern Seite erbohen/ tumb mit J. H. S fovon einem Rorber:-Eranst umbgetten/geseid) net.

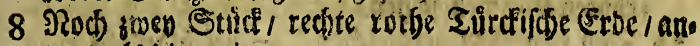
Der's als bie vorigen.

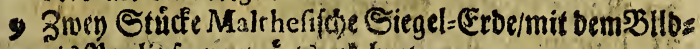
nis Pauli/ fo vorwånts gett bret.

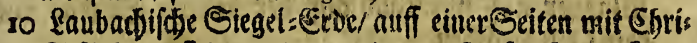
fti Sillo/ auff Der anbern mit ciner $\Re$ ofen bemerdet.

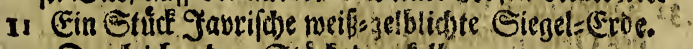

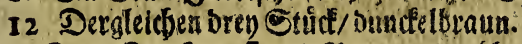

13 awey Stiucf we fle mit Bergen Dariber $\odot$ uno D

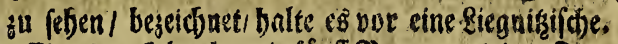
14 Sine Dundeetrotbeimit funff Dergen uno bren Sannen: इ̧áutnen benterder.

15 2wo weiffe mit eilf $\Re 5$ 


\section{耀 $(154)$ 耀}

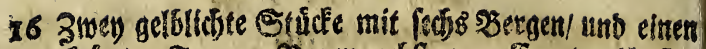
getrinten Tannen=23num nebft gtwey Ereutrmeife fles

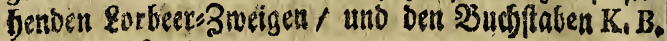
$165 \mathrm{r}$ gejecidinet.

17 âter Dergleiden Violet blaue ${ }_{16} 6$.

18 Eitue ganks weiffe bon 1605 .

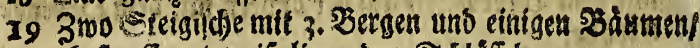

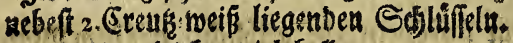
20. 3wen bergleiósen viel beller.

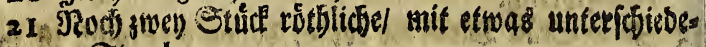
nen Siegel.

22 3wey Stude feuerrothe mit II. S3ergen/ und, cinen Şaum bemercfet.

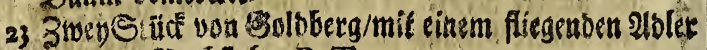
unt oen Buthifiaben B. T.

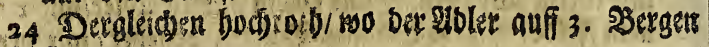
fetert.

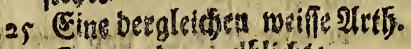

263 mo andere aelblitite.

27 Steignifre Eiegel=errbe weiffer als bie obige?

283 noo rotbe gefiegelte Exten aus $50 ̈ 5 \mathrm{~m}$ m/ von Anno

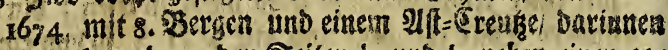
w. m. be unb an ben Seifen k, und 1. neben ciner ges crouten 8 lumen foben.

29 3mo bon then dergleichen Stempel blauliogt meip.

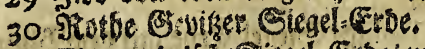

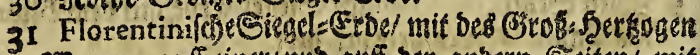
waspen auff einet/utio atef ber anbern Seiten/ mis Dem Orleaniforen.

32 Dergleitben yier estude / auff Det einen Eeiten / fats

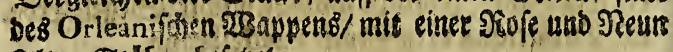
Bilien. St? ben beferget.

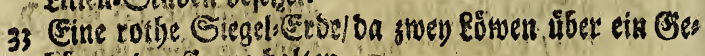
birirge eine Crone balten.

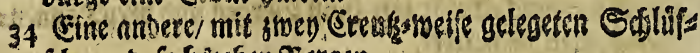
fela/uns fedjegefer \$ergen. 


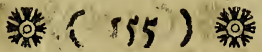

3) Deraleicien gants weip.

36 Derile: ben aelódidt.

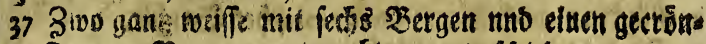
Sannen Baun/von fer bbigen unteríchieben.

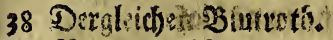

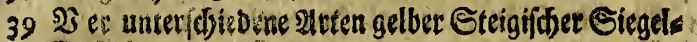

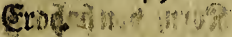

40 Eine sundelsbraune Eroe mit 9.53 ergen uno ro.

meiffen Sdyúfifeln unb Drunter: Terra Sigill.

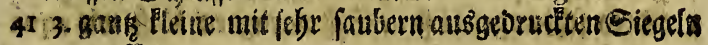
graue Ẽroe.

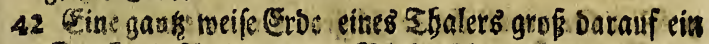
Stod mit SRojn um den SRanot/ Terra Sigillata No. barfowenfis itoo.

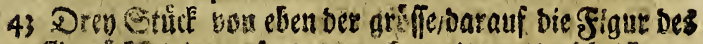
Gemady es barauf es gemadgt/ mit ber um(a)rifft:

Foffile Arborefcens Maslenfe Kleinichweinnenfe.

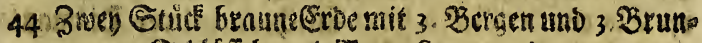

nen ztorn Sdgliff la huno Terra Stregomi.

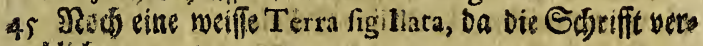
blichen.

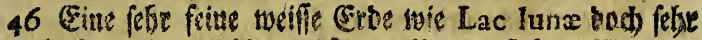

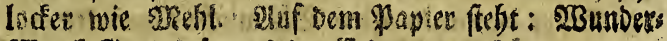
25sta Eidtes fo aus Der (Erben gerwadsen.

Der Jroenter 2lbtbeilung

son Dee

Finffen Sronung

Snotret Lunterfociot,

Scált infich

Die Etrite,

Lino stwar Enflicis:

Diereselen etent

unto sus folthen

Die burchfiditigen / fo mobl Orientalif be als

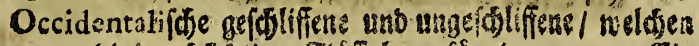

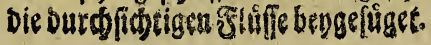

$x$ ein 


\section{然 $(156)$ 就}

I Ein von ber Natur oben uno unten gleicfani Eantide ge(d)liffener $\Re u b i a / n o d)$ rof.

2. Ein Elein' gef(d)liffener Orientali ider Sapbir.

33 Finfi Orientali jde ?tubinen.

4 Ein Orientalifđer Syyactintb/etwas blaß.

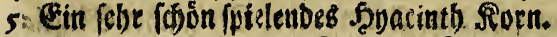

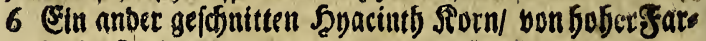
be fonft Chryfolit genanat.

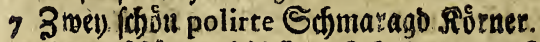

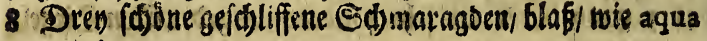
marina.

9 SBier Orientalif(d)e gefostiffene Granaten.

10 Ein groffer Amethift.

II Eir gef(c) liffenter Eleinerer Amethir.

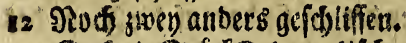

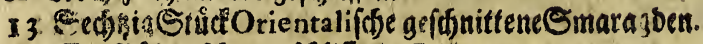

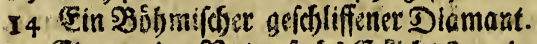

15 Ein vort ber Natur fedse Edildyt formirter Diamant! obrweit \$yrmont gefunben.

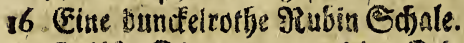

17 Fali(o)e Diamanten aus Der Sa)meis.

18 Ittoere von einem anbern Dre.

19 Einiye accommodirte 250 frnil(đ)e Granaten.

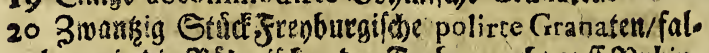
len wee bie Sbibmi(d)en ber Garben nad)/ auff Siubinפ্lth.

21 Ein znm \$et/daffit accommodirter/ etwas getblidft fallenber Diamant.

22 Sbimifide (Sranaten.

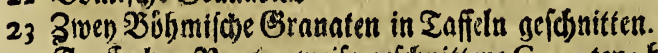

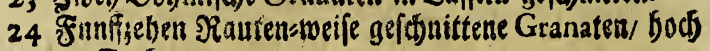
yon Sautbi:

25 Bin Stúc Orientalifoter Caicedon.

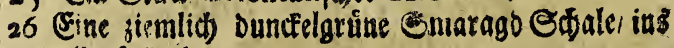
gelbe fallent.

37 Ein Stúd Orientali ifort Topas. 


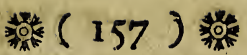

28 Ein Stifd gefolififener Lapis d'Aventura.

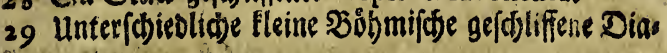
manten.

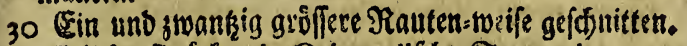

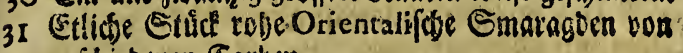
berithiebenen Farben.

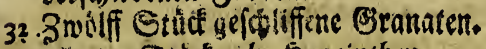

33 .

34 Drey tobe Siubinen.

3f Drey robie Urientalifore Smaragben/siner bund'cer an farbe als der antere.

36 Pebber Orientalif(b)er Amechifften/ in Der \$utter ltegrnd.

37 (sin groffer unreiffer Orientaliforer Estannt/artig b)abrundt genadifen.

38 Orientalif(de Sinptir mie fie bie Matcrialifen jur

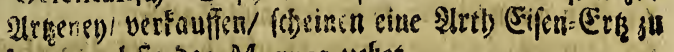
fenn/ inell fie Der Magnet gleffet.

39 E:in Strúf rob Aqua marin.

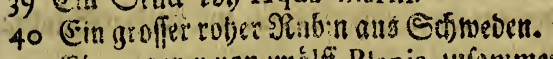

4I Ein anierer von jwoulfi Planis, sufanmmen gefergetet.

42 E:n Stúf Calcedonier.

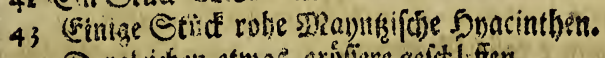

44 Deraltichen efwas grúfiere geforl fen.

45 शimuereforticher rober Diamant.

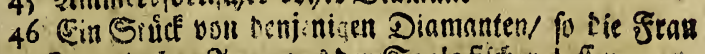

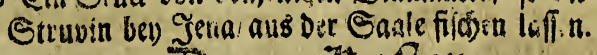

$$
\text { INer } 12 \text {. Elafren. }
$$

47 Sinize groufe Frenburgilde unpolite Granaten. 48 Ein Stutd Occidentalifíder Damantuten.

49 Eine Amethifent: Strufie/ nit Siryflallen.

so zwery Stǘf robe Aqua marin.

SI 3 sinige Amethiften.

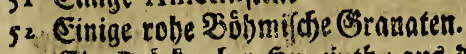

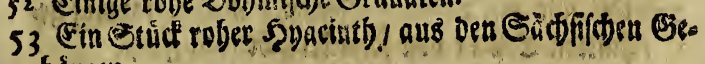
burgena 


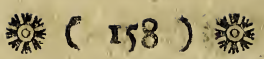

54 Dergleiden / wo ant ber Seiten etwas Smarage ate madien wollen.

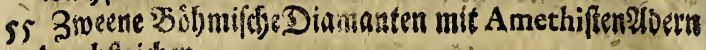
ourduftidien.

56 Einige Studen Amethifitt.

57 Nods einige Differente.

58 গod) cine faubere Amethiften Stufie:

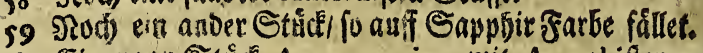
60 (sin yaar Stutf Aqua marin. mit Amethiften bermifoit.

61 Ein losin Stût Smarago/ zienlib) grob/mit Ame-

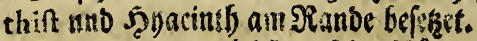

62 Sin anderer sergleiden/ rebe fojoner.

63. Zwo flinere etwas ioflediser.

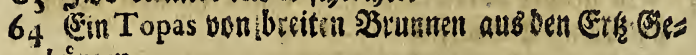
bưrgen.

6r. En gank blaffer fyatintb aus Sachen.

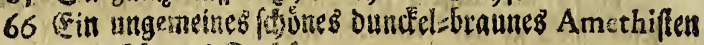

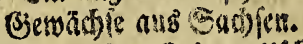

67 Fin rober Orientalifoter Topas mit rotben Sulmber umbleaef.

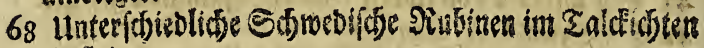
B)eftein.

60 3abliţer rofie Oeanaten.

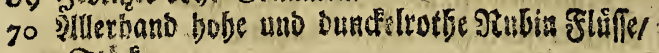
5. Stitie.

71 Sin Amerhiffen ofu?

72. Fainfi bohe und bundelfarbige Saphir=Finffe.

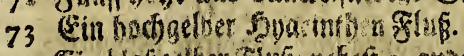

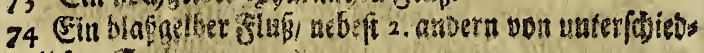
liden forarsen.

75 Sier gefdliffene Crnfallen / wie saffetrouten und Dergleici)en. 76 Ditn Saphir=5shid?

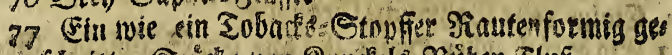

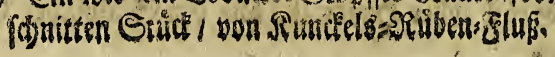




\section{粼 $(160)$ 繗}

13 Drey Orientalifofe Opal.

14 Ein gtuffer Opal you den Carpatifjen Eebuirgen.

is Bmeene Carniol Sfleifhfarben.

16 Zुweene anbere/ febr lyodh von Srarben. Gin Ropfi

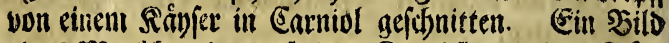

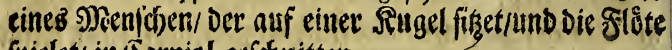
fpielet/ in Earniol gef fonitten.

17 3weete gant gelbe Earniol.

18 Bwen Cam huja, in Deren eines ein Ropf eines (5) gelbs in Dem andern Des Alexandri Magni Sovuff gé (id)nitten.

19 Ein Sardonix.

20 Ein balb=-iurchficffiger griner sajpis.

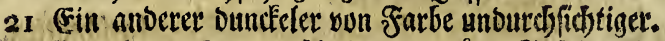

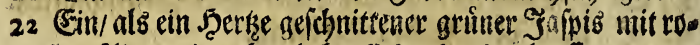
t)en 2loern/ Dancben licbte Calcedonier Lauffen.

23 . Foth uno griner Jofpisis.

24. 3iven gan totbe Etrice Safpis.

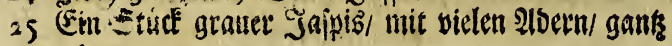
rutben.

26 Ein fo genanntes Beli-2luge/ unten geto uno balb.

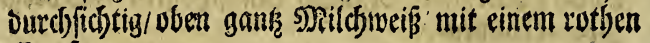
Erenfs.

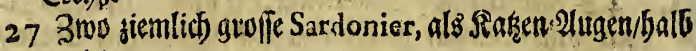
rundí.

28 3ino andere fleitnerer.

29 Unterichieblide Agathe, ba immer einer anders yon Grutbe/ als ber antere.

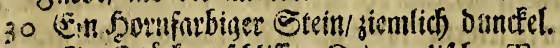

3I sin Et tid aefólifien Orientalifber Topas, mit Sildjfarbenen/ unb rothen Ibert!.

32 Unterifbieolicbe rotije stgathen/von verfobiebenen garben.

39. Ein fobin Ettid Orientaliforer Sappis.

34 Ein Etwite rober fehr lyeller Calcedon.

3 i. Sin anderes bunckeles. 


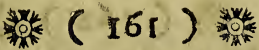

36 Pramnion five Morion eit fotwarfer Erifall vor Wlaniz.

37) Ein Stư Duncfel Topas.

38. Cin Stitid Carniol, fo man bey Diben gefunben.

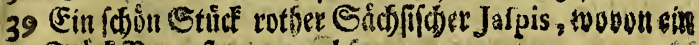
Stuid 'Baumfter gemachien.

40 Ein roher (Earpatifider Opal.

4x (sin Saidfifider Hyacinth.

41. En Saldififiter Topas.

42 Dito. 43 Dito fooundilet.

45 Chascedon van Planifz.

Durdfitotige Croelgefteine, Orientalifobe und Occidentalijthe gefolieffene und

\section{ungejolitieffene.}

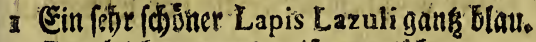
Derglcichen etwab weif vermilost.

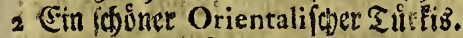

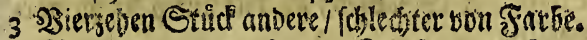

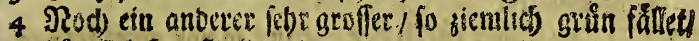
fóalt fidftbar Cold/ woie der Lapis Lazuli.

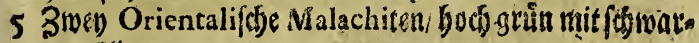
ken sloern.

6. Eingrofier Malachit, tiber ein zoll breit/ uno ein balb

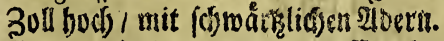

7 Ein Gamhuja, woraus ein Engels= Ropfif che fauber gefocuttten.

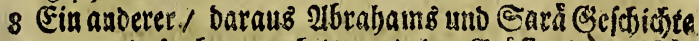
ungentrein fauber gearbeitet / in Der Gruffe eines \$fefo fer=fiorns.

9 Ein Orientatiffer Agath, barauff weiffe Ploern eine $\mathfrak{B e f t u n g}$ astig abbiloen.

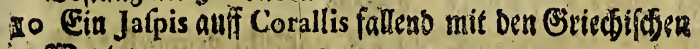

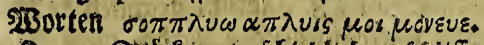

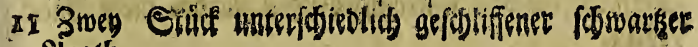
सhath. 


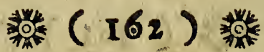

12 Ein grưner Safpis mit rotben \$uncten/ Darauff cin Delphín fauber gefónnitten.

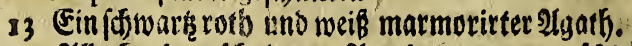

14 Illerband gefdonittene 2lgatben von unteridjiebenen garben.

is sin io genaunter Srovten. Stein / runo unten etwas bofl.

16 (sin anberer ablanger/aude efwas bobl.

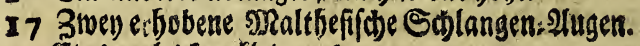

18 Ein Dergletdent fleineres?.

s9 3wey Deraleicifen ganks platt.

20 Zweey grófifere/ beller yon garben fo man wegen bes

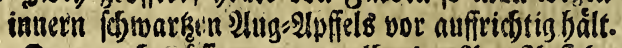

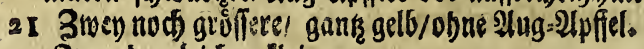

223 wery Dergleicisen tleinere.

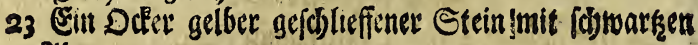
थbern.

24 Ein blauer Etein mit einem meiffen ßinge umgeben;

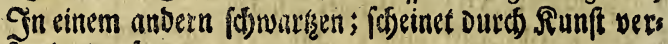
fertiget su fryn.

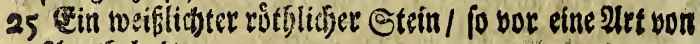
A a at batte.

26 Swen Hleine 21 ter: Steine.

37 Sinj (otsarser platter Etem / wie ein Agath / barauff fiib bie Distalle proven lafien.

28 Lapis Lazuli audis rob.

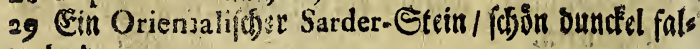
Leno.

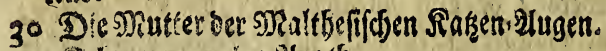

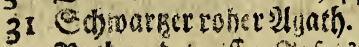

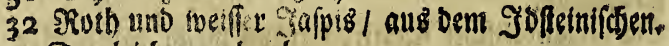

33 Dergleiden nod rol)

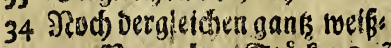

35 36. Decunjeben Stuid/8. Eantidot gefolieffene Ges

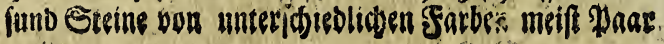
meile. 


\section{耀 $(163)$ 䊁}

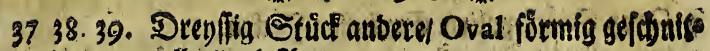
ten / bon illetband $\mathfrak{A t}$.

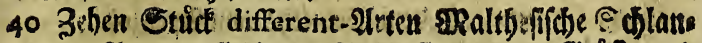
gen = 2lugen allerband Ant 1 . Couleur, Groffe und Beffalt.

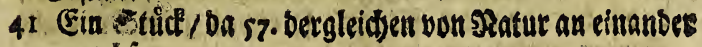
gemadjien.

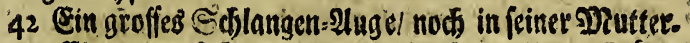

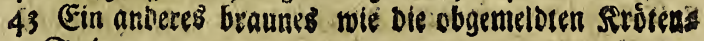
Steine.

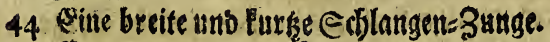

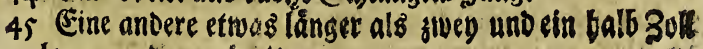
lang / uno zmey freit.

46 शrod) etme andese (a)måres lidje.

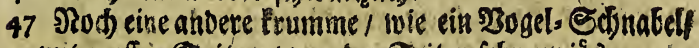

mit gruffen Spisen / van ser Seiten febr cutriós.

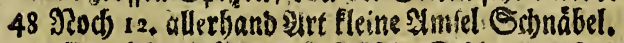

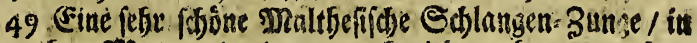

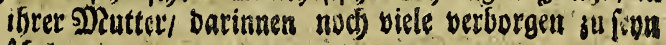
侮einen.

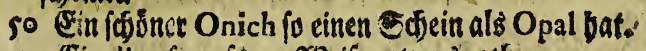

SI Ein dito fo auf oem গ̇seifen etwas totth.

52 Ein weiffer fäubeter Stein mir rothen unb weiffer Sinien.

S3 Sin febr jouterece Bleitreb ovales Steinigen / fo als ber

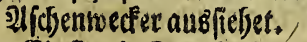

s4 Ein Lapis Camahuja, unfen borufarbig, oben weifs. 55 Dren Stúd rober Carniolo

56 Ein fofinet Onich.

\section{1wenten:}

Steine $/$ fo wegen iffer allerband befondern Beffalten aufgebobien werben. Die rr. Eduublade.

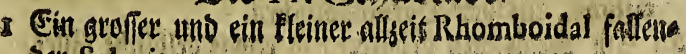
Ber Selenit.

$$
\text { 2 } 23 \text { ameg }
$$




\section{粦 ( 164 )}

2. Bmen Stide politete (Eryffallen 3acken.

3 Ein allesect Rhomboidal fallender Selenit, alts Dem

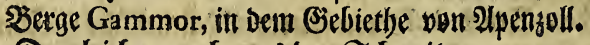

4 Dergleichen audch aus ber Echmecis.

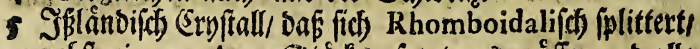

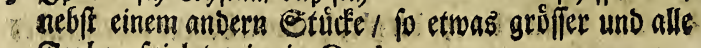
Jarben fpielest twic ein Opal.

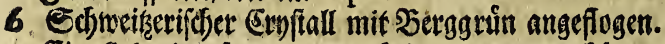

7 Ein Selenit, io artig burcheinantber germachjeal aus

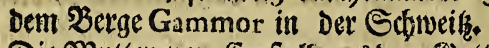

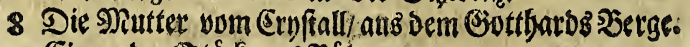

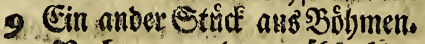

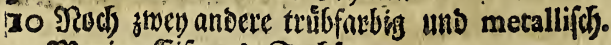

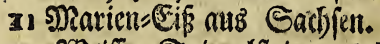

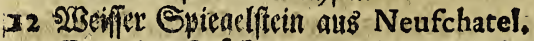

$x_{3}$ Gin ander Strite aus Sadjjen/fo Durres) meinenboennEpiegel calciniret.

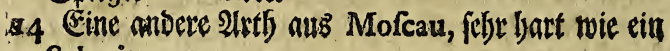
Selenit.

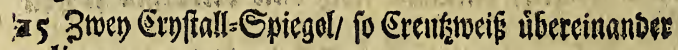
liegen.

1n 6 Durcffictstiget Selenit. fo Rhomboidal fâllet.

a7 Ein Mineralifolet: Calcedon, alks Rothsmund im Berner Gebiethe.

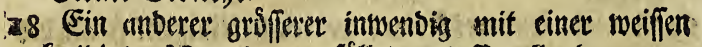
Freibigten Materie angefífllet/ auts Engellano.

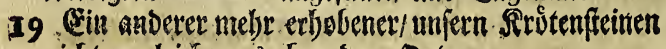
nibbt tangleich)/ aus eben Dem Drtes.

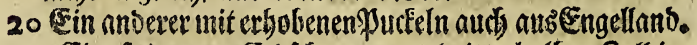

21 Gin fteinerner Evoichmamm / Dritte balben 3olt im

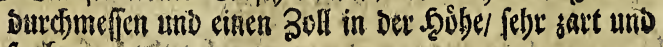
4 fruber.

22 Ein fiv genanater fóswartser Hyftrolithos sber Muts terffein/ exhbens utriusque fexus Pudenda accuratiffime, sus Den Darmoftátitichen.

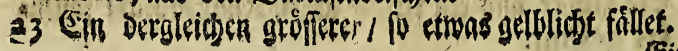

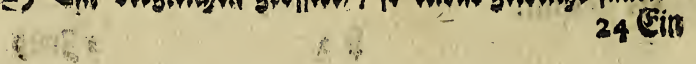




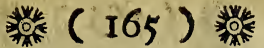

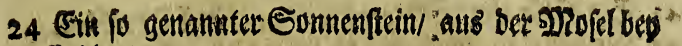

Coblents.

25 3wo moere/ anbers geffaltet / auff bunte Eteine ges swadjen.

26 Ein brâunlidber Sirotenftein/ von siemlid)er Grivife.

27 Ein anberer bun eben ber Farbe/ nebof einem fo mebe meip fállet.

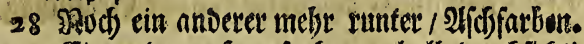

29 Ein anberer frornfarbener/ balb Durbsfichtig.

30 Nod) ein fleinerer braunlidjter.

$3^{1}$ Ein anderer ziemlids) platter/ Blaulicfjeter Couleur.'

32 Ein anderer 10 gleidjfam einer verfeinerten Dousbletten gleidfểmmet/ wirb Dods son vielen mit unfer

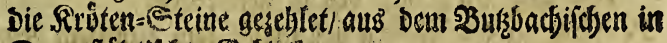
Sarniffádtîfoben Eebiethe.

33. 3wo Grepaudines, Davon ciner braun / Der anberee

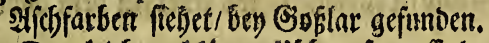

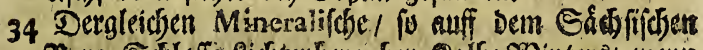

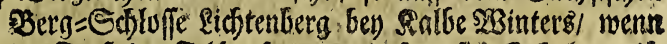

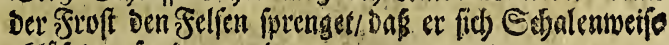
abliset/ gefunden werben.

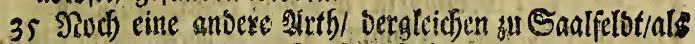

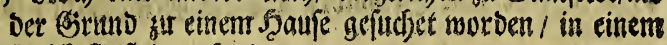
Talté= Beffein gefunden.

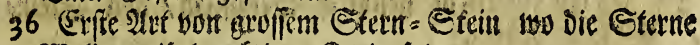
23sellenweife bures osn Etein fersen.

37 (Eime nutere 21rth) wiecine lyalbe Rutgel geformiret! mit groffen Sterner.

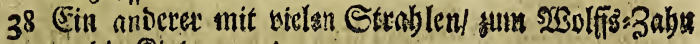
vor bie Sinder aptiret.

39 Nod) ein anberer mit Eleinern Sternen.

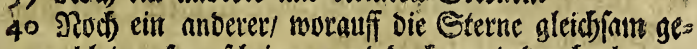

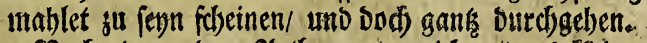

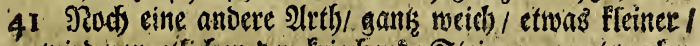
twirb von efficken der friedjende Stein genanut/wetl er im Esige bin uno ber geljet. 


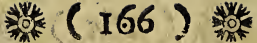

42. Ein ablănaligfer Etetnftein / mit fúnff Strableb.

43 5?:0ch ein ancerer (éber fart/ mit vielen Strablen.

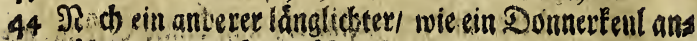
jufethen aus Dem \&egertióden Ferge.

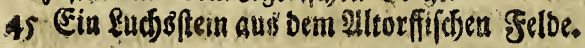

\section{Dov I2. Kafter.}

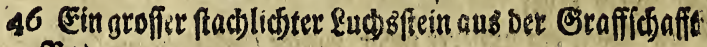
Babea

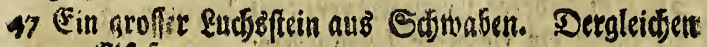
ous sirap.

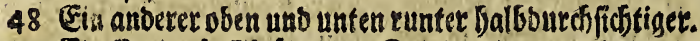

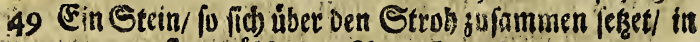

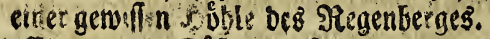

so Gin mue ein gáággen gefaiter Êtein aus dem Regrtio fiton $\mathfrak{b} r$ de.

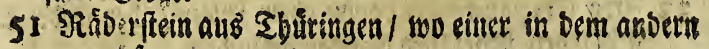
gematifen

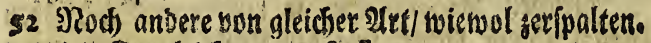

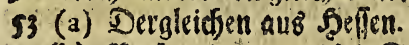

(b) Nod) anberer aus Der Sdimeit.

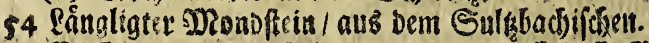

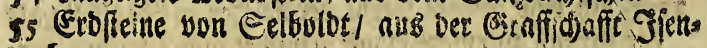
burg.

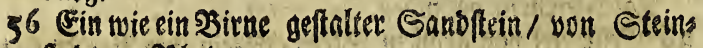
frabt am Ratein.

$\$ 7$ 3mo Sirien vom Lapide Nephritico.

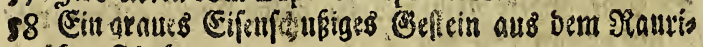
idszen Stabl.

59 Jubenffein / fo siemlid grof / been Dber Eobenffein ges funber.

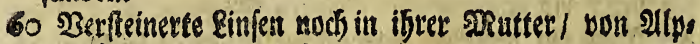
Gebuirgen aus ber Edweris. 6r Deraleiden aus ibrer Diufter.

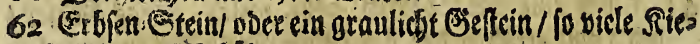
feiffeine in riof bălt.

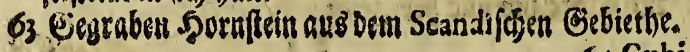
64 Cubi- 


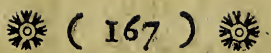

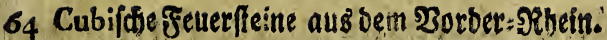

65 Specffern aus Chira/ weldben vor eine Mrt Lapia dis Nephritici balte.

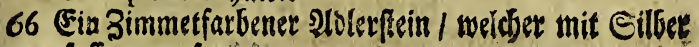
geforfet getwefern.

67 Eir anderer gantsotber.

68 शtode ein anderer ablanger.

69 Ein anderer gruffer fehr rots.

70 Einige fetinerne soburffel / wie fie bey Baben gegrabet werben.

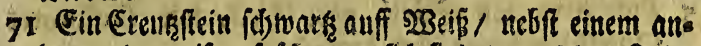

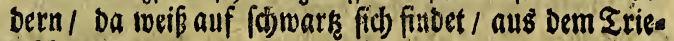
rifojen.

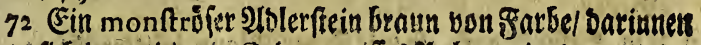

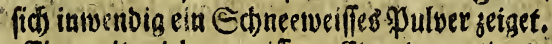

73 Ein mit vielin weiffen Eirculn artig tumbzogener. Stein.

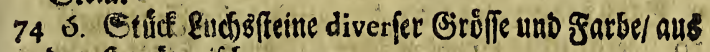
Den Staniberif(d)en.

75 Ein fChóner Dundellbrauner Sirviten-Etein.

76 Ein dito granter.

77. Ein ü Siein geworbene \$Ranvel mit Der blauen eys permen Sdiole.

78 Eire enviófe Singel fo ben Dem Dorff Shippen gefuns Den / welthe in 2Baffer serfipringet/ biervon ift eine bes. funbere Defcription.

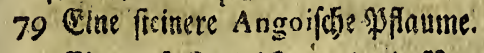

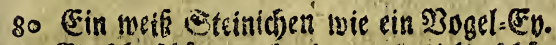

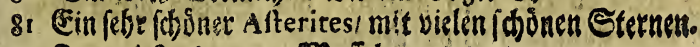
82. 3wey Afrerites yon 5 Ruffet.

$8_{3}$ Ein feiner $\mathfrak{B}$ undffein bon \$Ruffel.

${ }_{83}$ Ein dito ba ber 3 und von Eifen.

84 (Fin befoubere $2 / 2$ th rotb Eurafl.

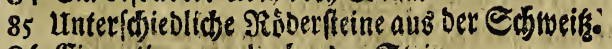

86 Gin artiget ovalet bunder Stein.

$$
\Omega_{4} \text { Drit }
$$




\section{漛 (}

Pritteng, afferfand andere Steine von

Marmor, Yysiten, Speclifteinen/ uno

Dergleidien.

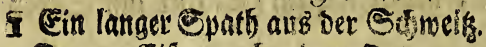

- Geauen Eíf bon eben bem Drte.

3 Eizartiger Spatt) mie Gerraben Elffenbeir aus bem

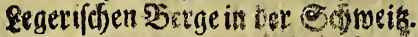

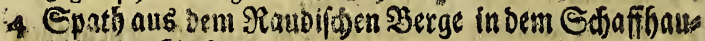
fijoben tsobietbe.

5 (cime 2lrth yon Gyath boer Esips/ mie fublimires Duedfilber 1 aus eben Dem Drte.

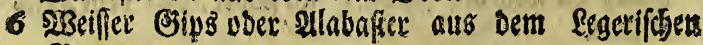
Berge.

2. Ein Stüd Epatb/fo nie Fecber alaun fplitteribjt ged wadiferi.

8 feber Allaun giemidis fort.

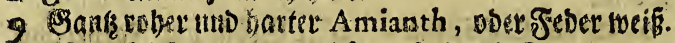

3o Derglichen etwas weicher / fo bura feuer zu einem weing nqulber gemadet mitb.

1) Gruizer Arnianth. fo bas Fetuee leiben lan.

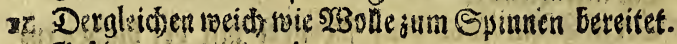

33 Gefponinen Amianth.

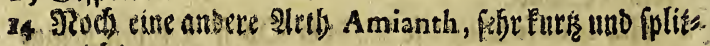
serid)t.

is Brinntidfiter Amianth auts Gerantuinder.

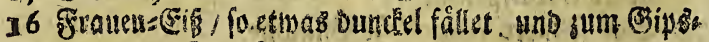
şremen gebrautogt miro.

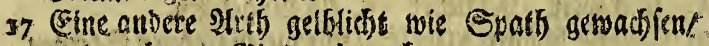
woro anch sum Bips gebrautbit.

18 Cine artige Stuffe yon roth uno meif Frauen (Sifil

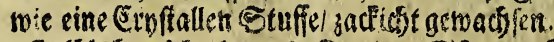

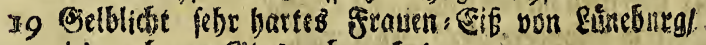
twiro auch jum (Cips gebraudet.

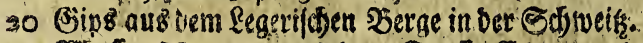

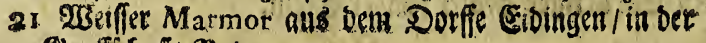
Scaffidanfit כaben. 


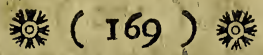

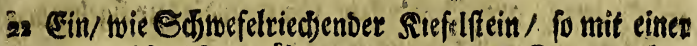
merallifden Saut überzogen / aus ben Frenenwaldiphen Ge funto Szrunnen.

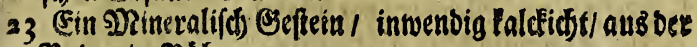
Baber in 30 d́fmen.

24 Ealdichte Sesfetix aus ber Elbe.

25 Ein mit Ochra úberall iberzogener Stein / aus bem Edimalbadifiden Saurer= Boumnen.

26 Reberfteine aus ber Ediweirs.

37 Ein Strid Etein aus Der Jnfiul Galtha im Tivtrenis

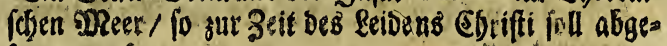
jprutugen feyn.

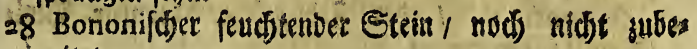
reitet.

29 Sier ectidfte Etcine / bon manciertley \&arben / aus Der See bein Puteoli.

3o Eine lobmarge buncelele Materie/ gleidjam wie buto

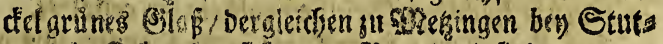
gatro in Edswaben fich ganke Szerge voll finter.

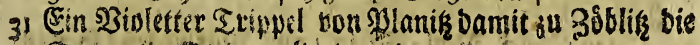
Eerpentin eteine politer werben.

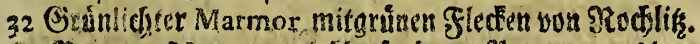
33 Brauster Marmor mitt blut farbenen \$uarten/auB Ders

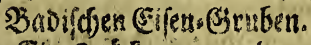

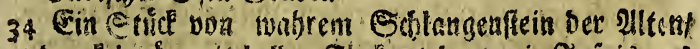
Dunckelgrin mit bollen ficteten/ batt mie Japipis auts

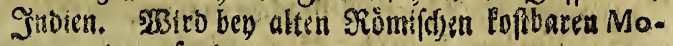
numenter gefunder.

3) Ein artig. Beffetin mit fojwartent Rinien umgogen/ aus vem Siottifarbts:3erge.

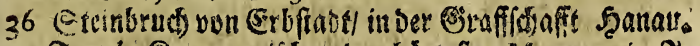

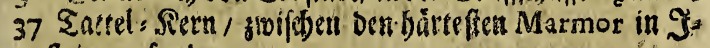
frien gefunben.

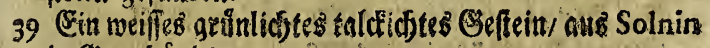
in Straubinoten.

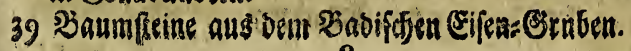
\&\& 


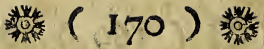

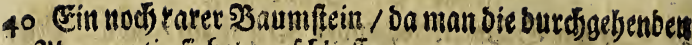
Ho:rn artig fichet / aefólieffen.

4 I Zw'l fofone 3aumftetue / Darinnen braune 20ern bif 20lden voritellew/ aus ber Siauk.

42 (a) Storentmiffor $\$ 3$ aumflein.

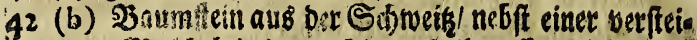

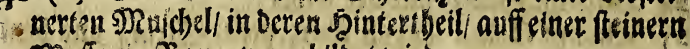

Nafie ein Baum borgebiloet wird.

43 Cin Duars aus bem Saure Sachseu zu Saalfelbtl. barinnet no fid bie Rliffite abfegen / ess als tleine

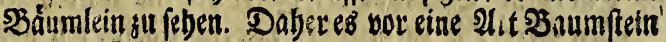
pasfiern tan.

44 Serpentinftein / Doer 3edliţifder Marmor. Niole Stein / fo qut riechet.

45 (Ein moblriechender eoher Riefelftein.

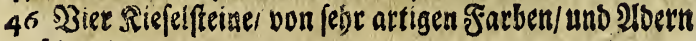

ithattirt.

47. Ein Durdfichtlger Siefelftein als ein En geftalret.

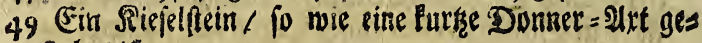
fialtet iff.

so Zney Stuid $\mathfrak{B l o l}$ Stein / ans Norwegen / fo nod frarcte riectsen.

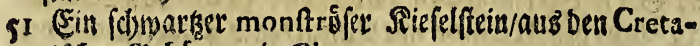
cifoben Eiebárgen in Champagne.

52 श2od einige Stlide von roben Gofteinifor)en Marmor.

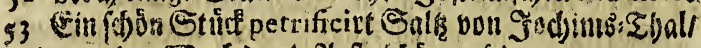

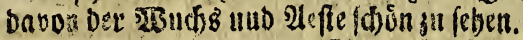

45 Dito von Keipkrig aus der Eand= Birube.

46 Dito eflwas amoers geffalt von dito.

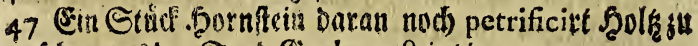

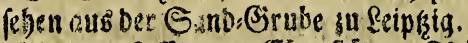

$4^{9}$ Lignum foffile von Eiperfitot in Shuringen.

49 Dito vou Reipsia.

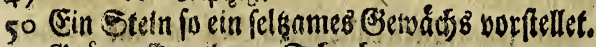

s) Gruine: Spatt vor Sobneberg.

fl Ein wiffer Marmor bon Zunnuerg. 


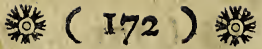

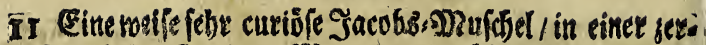
Brestlideu freinigten Materrie genwadfen / von eben Dem Drte.

I2 Ein Evofarben Gesfein / bariunen fiob eine bunfe SPRantel mit peiuen Dhren / febr artig absebilbet.

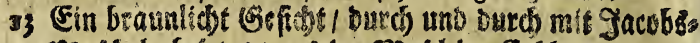

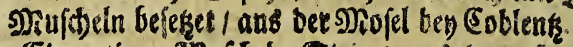

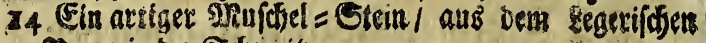
So erge in ber Stwiveif́.

I5 Ein anderer mit einer beevorgeberben Epige vers feljen.

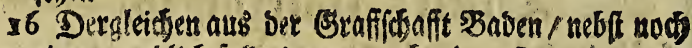
einerm runblids falienben / bon efen tem Drte.

27 Dien bidfe / getiupte/ auffer braun und intwentig

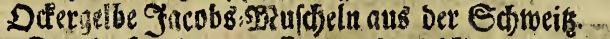

18 Dergleifien bren groffe $/$ und fo biel lleine $/$ aus einem

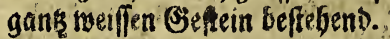

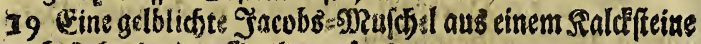
beftefent / bey Esoslar gef untben.

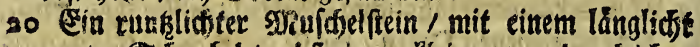
rmaten Scinabel / nebff tinem Fleinen von Dergleidfen 2(trth.

2) Cine getippte Sacobs = Mur(bel sientlid) platt / aus Neufchatel.

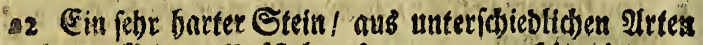

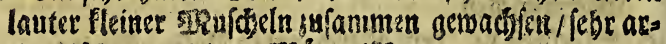

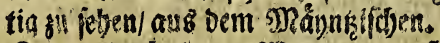

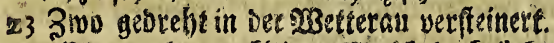

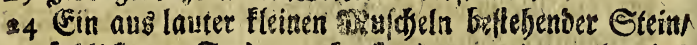

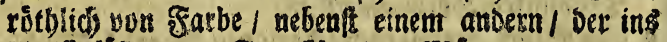

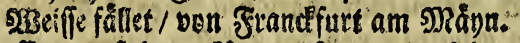

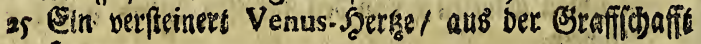
Sanau.

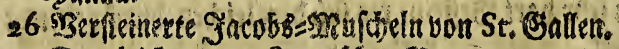

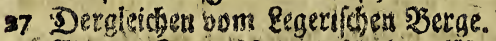

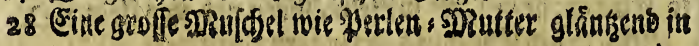




\section{紫 ( 174$)$ 耀}

go जeatn verffeinerte bon eben bem Drfe.

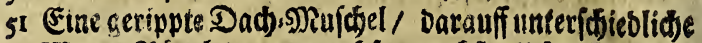

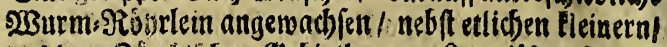

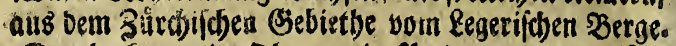
S2 Dergleidenen mit Obren mie Perten s Mpurter gläns Bend / aus Neufchatel/ utad Dem Serner: Gebiete.

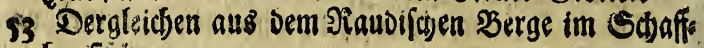
baurfition.

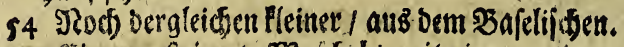

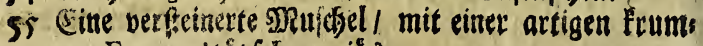
men Extremitốt fehr curiós.

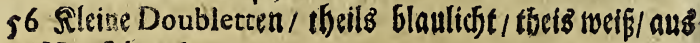

Neufchatel.

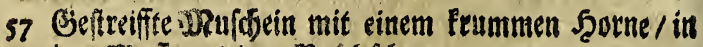

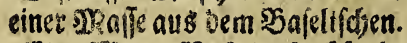

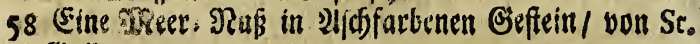
(siallen.

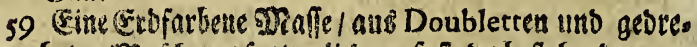

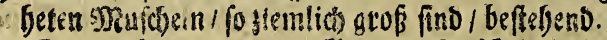

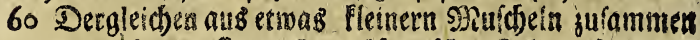

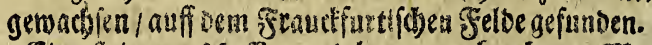

61 Eine fteinerne Maffa, aus tauter serbrod)enen Mn.

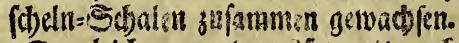

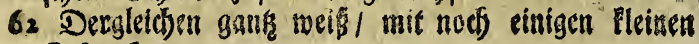
Scinerten.

63 Ein berfieinerter Esaper aus Neufchatel.

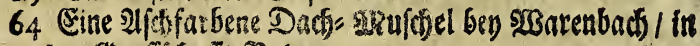

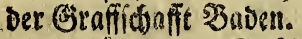

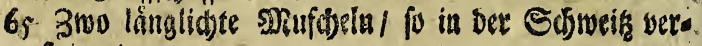
feimert.

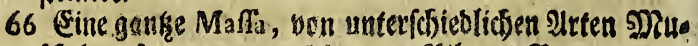

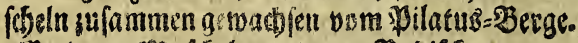

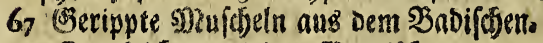

68 Dergleichen aus Dem פerniichens

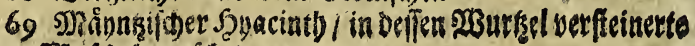

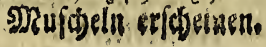




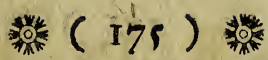

7o Stride von einer verffeinetten Dadj:Mnufosel.

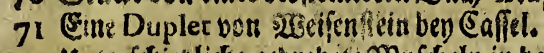

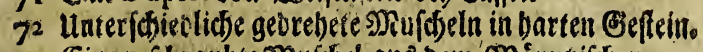

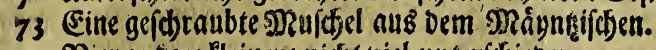

74 Sier andere Eleinere niff́t viel unterf(chieven.

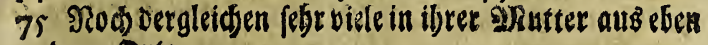
Dem orte.

76 (Sin 2 Rufdolffein 10 ungemein bart unv aus lauter Eleinen georebetern Mhu[weln beffefet.

77 Sin verffeinertes Hleineres 3rompet.5̧orn/ mit fobe wiel anbern / in einer gelben Maffla,

78 Dergleiđen Maffa mefbrbicfer / bårter uno meiffer.

79 Dergleideren nit gank Heleinen glänţenden Zrompets Şorns / van St, Gallen.

80 Rleine Erompet = Şorns/ fo von biejet Maffa abs gefallen.

81 Eite verffeinerfe CEtbe / aus einer gebrefieten Diujdel/ febre artig zu ficher.

82 Serftetinerte Trompet, Sorns won St. Gadlen.

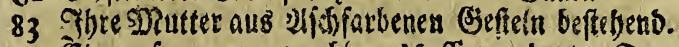

84 Ėine sufammen getwactjene Naffa von lauter Srompettsfjoinceens ben srandf furt gefunber.

85 Dergleioben twenig unterfibieven.

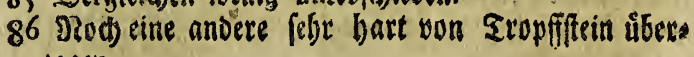
zogen.

87 Mi Bjeftein / aus ber șetterau.

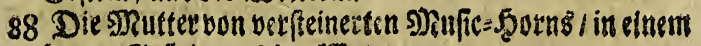
Garten Berftein aus ber 2 Detterau.

89 Dergleiden granticht wie eine EHfen= Stuffe.

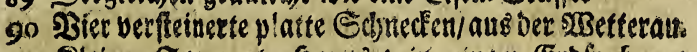

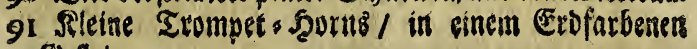
Geftein.

92. (Eine Dergleiden Maffa / fo ber worigen fegre nabe ESinmet.

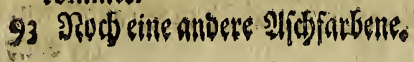




\section{紫 $(176)$ 褛}

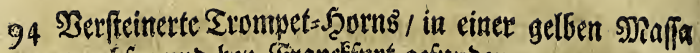
gemadifern und been Strancffint gefunden.

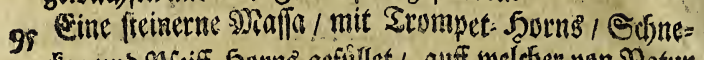

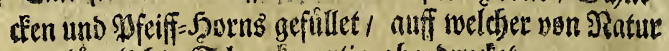
eine línglitifte Sdonecte attig abgebructet.

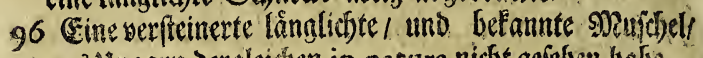

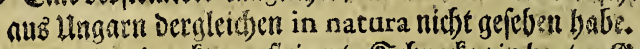

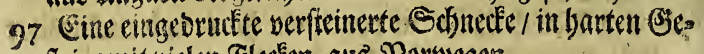
fiein/nit vielent Sflectien aus ఇeorvegen.

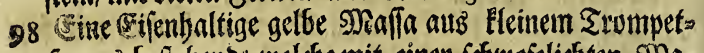

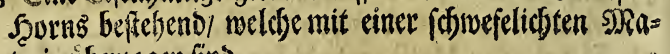
terie cifersoger fint.

99 Dergleiden mebr weik/in buinneren Sdjalen. 100 Eine anbere SMaffi bon gleicher Ult.

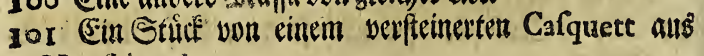

Neufchatel.

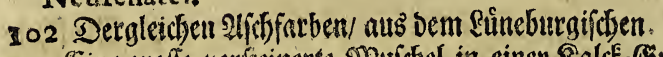

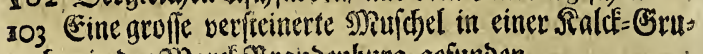

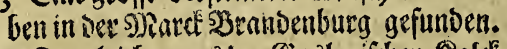

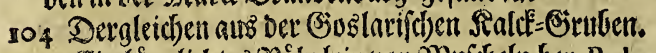

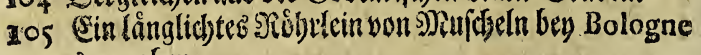
auşgegraben.

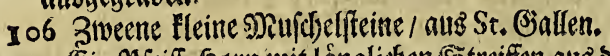

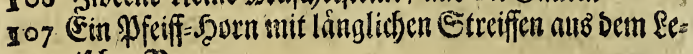
gevifífien SBerge.

gos Eine Ero = Sdinecle verfteinert/ fo ben פergen / in Der Graffictoafit Sannau gegraber.

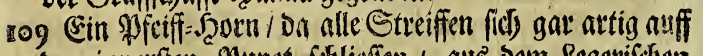
Dem innerfien spunct foflieffen / aus Dem Regerif(d)en serge.

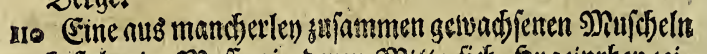

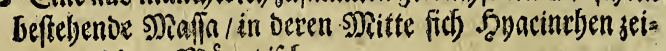

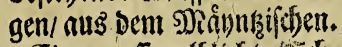

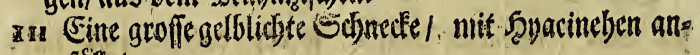
gefiultet.

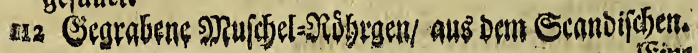

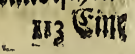




\section{柴( $(177)$ 耀}

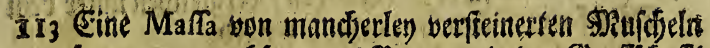

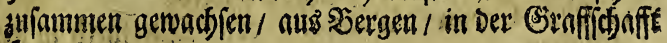
Sonati.

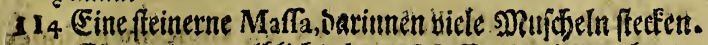
Is Sine anoere gelblichte borte Maffa; Darinnen lauter

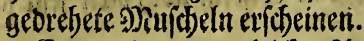

216 Eine anvere von gleidjer $240 t$ th

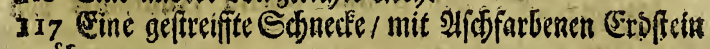
ilberzogen.

I8 Dergleidben / fo mit lnuter jerbrodsenen Doublettens Sctjalen tiberzugen.

ing Dergleidben 9 lid farbett.

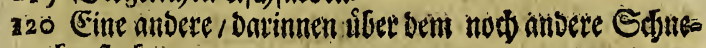
dien fredteri.

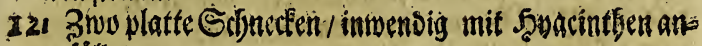
gefithet.

122 Eanglidfte verfecinerte Stfnedfen.

\23 Fine gelblidite Maffa, Darinnen viele fleine Srom: pet = Soirnb fredfen.

124 Eine balbfeinerme Stanede/mit Itou gefillet.

i2s cin gefteifftes verfteinertes \$feiff= Som aub Dem gerifchen Sictge.

126 Dergleicfeumit enet medicinifesen Setten úbersos gen oun siltorif:

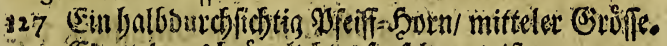

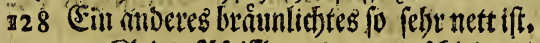

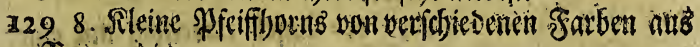
Stutgunioien.

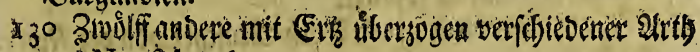
ans Neufchatel.

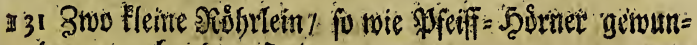
Den von eben Dem Drte.

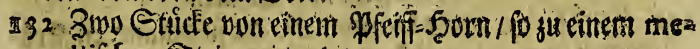
tallifínen Etcin gerwotoen.

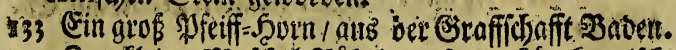

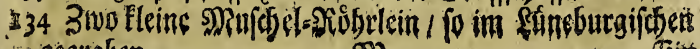

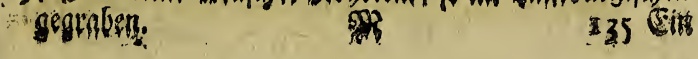




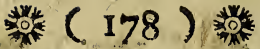

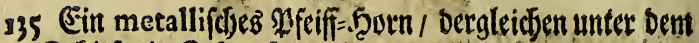
Ediefer in Sdjiwaben gefinten mito.

136 Ein Cornu Ammonis von Duerfurtl) in feiner Mitter.

\$37 Ein dito gejsjlieffenes / Da bie Concamerationes

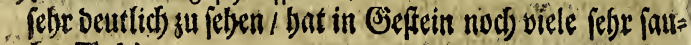
ber Tubinat:.

138 Ein Stuide yon eincm Corna Ammonis won Ejperftádot:

139 Ein Cornu Ammonis von Fundenturs bey Queriutth:

$14^{\circ}$ 5. Stiul geffreiffte Doubletten nus der Sdjweir. 141 5. Stuiff dito.

142 5. Stuid dito glatt won Donerfittif.

143 5. Stuik dito unterichiedl. Errófe von dito.

14.42. Stútú Bucordites von Eifencid).

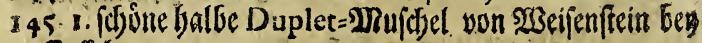
Cafiel.

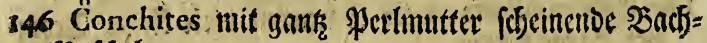
Minfibl.

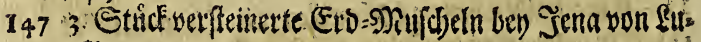
ter:Srumnen,

348 Eine Duplet von Sindel bey Eijenach.

149. 2. Hlein Duplet fo rieift nur calcionitt won şBenten: Gadi bey Durerfurtly.

150 5 . Etitfer accurat gebilbet als ein Umbilicus $\mathrm{Ve}$ neris, von Neufchatel.

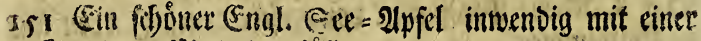
Etreibigten s) Jaterie exfiflet.

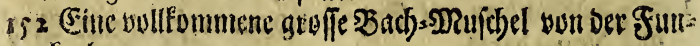
cfenburis.

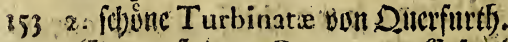

154 (Eine geffriemte Duplet bon (Erfurt).

iss (Bine idsone meite Duplet/ wie Die io genannten Sitg:

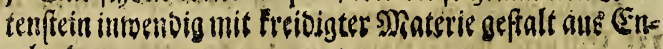
getailio. 


\section{紫 $(179)$ 悲}

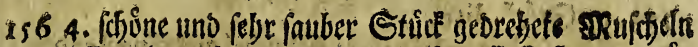

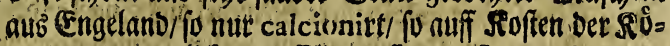
nigin Anna etliffe 30. Ellen teff gegraben wortoen. 157 ఇoods) cine ansere dito.

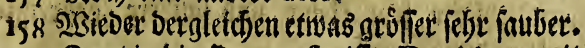

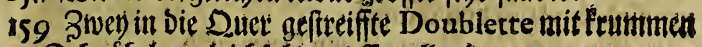
Edonábeln $/$ gleidjfol's aus Engerlanb.

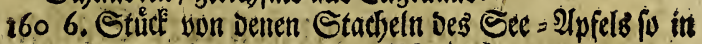
meififen Spatf betwantbelt find/ieler inuber aus Engel land. 161 8. Stinicf glatte Doublerte von Sena.

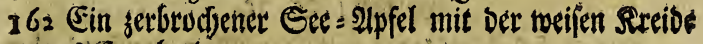
aus Engelato.

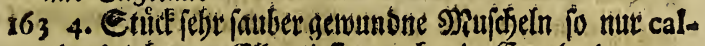
cionityt bey ;0. Ellten tieff gegraben in Engeland.

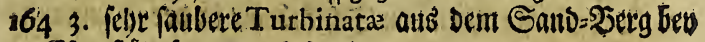
Es perffáoto io nur calcionitt.

165 3. Turbinate, fu petrificirittwon 166 (Ein Quadrat \$Muf(d)el butt Duer furth.

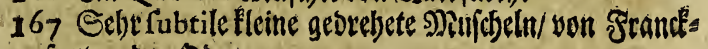
furt an ver Doer.

168. 2. geverthete s)

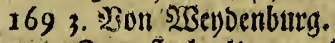

170 3wey fladse dito von 3ürith.

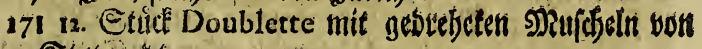
Stittgartot.

172 Eine Doublet calcionitt yoin ber 3eit uvn șseifent feein beri Eaffelel.

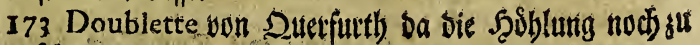
peheri.

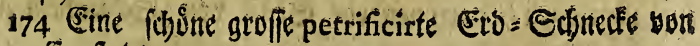
Einffabt.

175 Ein Conchites mit vielen feltramen sionudideln.

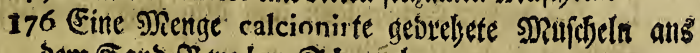
Dem Eanb=sers bey Sdrrajelau.

177 Ein Conchites, fo unten glatt intuenbion viel unges nein fifóne pechier jeciet won \$iaffel aus ber Edblefiert. 解 2 178- 


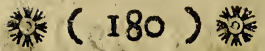

778 9. Griff dito.

- 79 Eine fobune calcionirte anufodel nuts Engelant.

180 Ein gelber Conchires mit vielen fleinen georelyeten

Edjneden von Frandfurt an ber DDer.

18r unterfobiedlidie felor fleine und faubere geftreiffite Doubletten you SRanfel.

§82 (Ein grof Cor Vencris von Duterututh.

Bwenteng, anoere verffenerte Sadten,

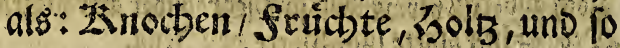
meiter/ $26,2 c$.

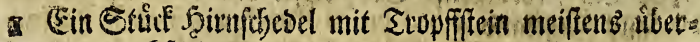

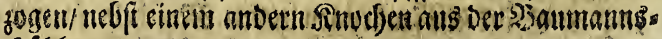
J78̊le.

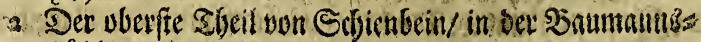
SToble petrificitet.

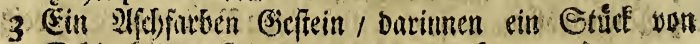
Sdjicabiein verfenert morben/ ats efen Dem Drte.

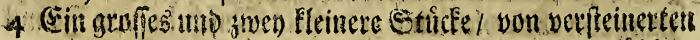

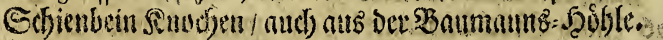

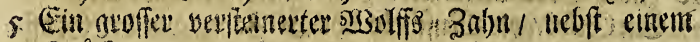
Stitce wen bergleichen von cber viem Dite.

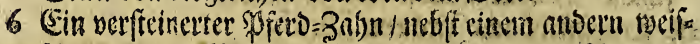

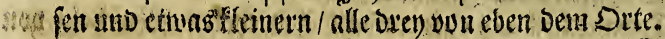

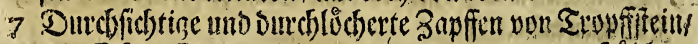

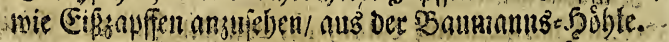

8 Der oberfe Etbil von Jufft: Snudjen/ an eben Dem Drte werkeinert.

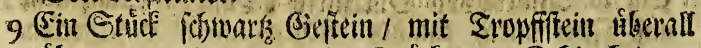
úberzogen / ontinnen sin Strid bon Enbienbein ju fichen.

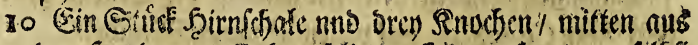

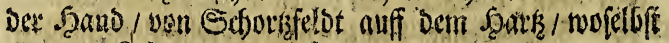
singath Scheleton gerwefter.

I1 Strols mit ciner freinigten shiaterie / úbersogen / ans cinem 5 bunuen in Engelland. 


\section{先 $(18 \pi)$}

>2 Segraben Einhorit aus ber Stbiutif.

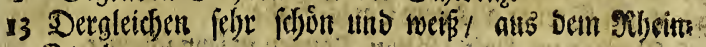
Etrobit.

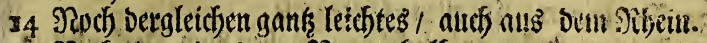

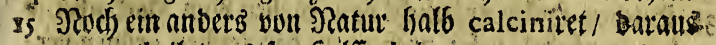
man urtijeilet / es fen) Soelffenbein.

16 Dergletdjen ballo gelbe.

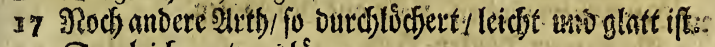

18 Dergleicjen etrơớ lánger.

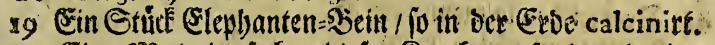

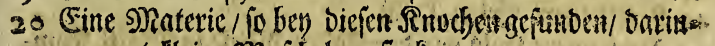
men ganks Hleine SRujhelgen fectien:

$2 x$ Ein Stuck gegraben Sçelffenbein/ attis ven Donau. 2.2. Sleitie verfecinerte Sinochen/ aus Earfinat in Ehwaben.

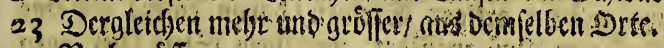
24 Nooch griffer.

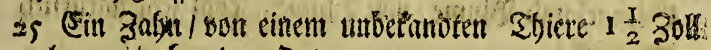
lang woir elecn Dem Drte.

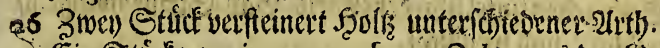

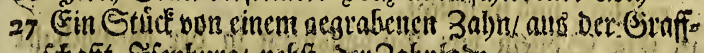

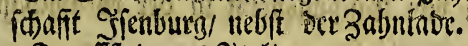
28. Sevuffifin ans Straliem.

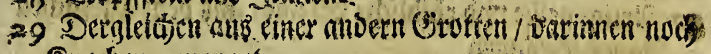
Sinodien gemenget

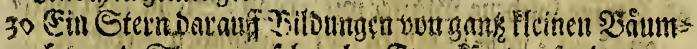

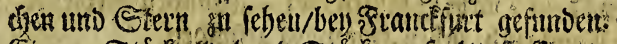

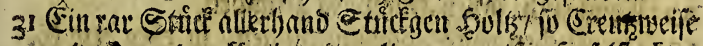

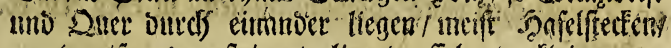
gants neip uno berffeincet/ / liegetsunf lanter flcinen ju=

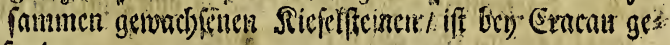
fumben

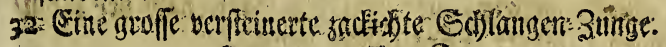

$$
\text { Der is. Zaften. }
$$

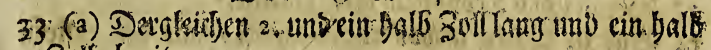
Zull breit.

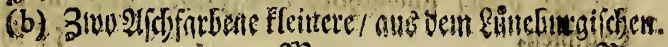

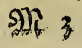
$3421 \mathrm{im}=$ 


\section{新 $(182)$ 䟿}

34 5unte 2 Imb

35 Ee:bE= Eteine nuz dem Carlb=sabe.

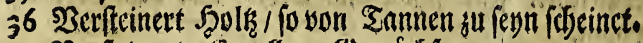

37 Berfteinerts Sorallen = Sicwódofe.

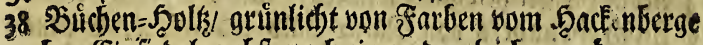
bey) Einfiebel/ nebft noch einem Dergleidben grümen verfteinerten Stúdfe / von cinem andern Dite.

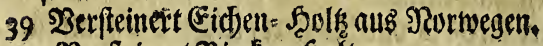

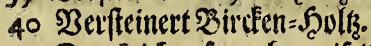

4x Dergleidjen fo mebr meis iff.

42 $\mathfrak{B e r f f e i n e t t}$ Erten= Solls.

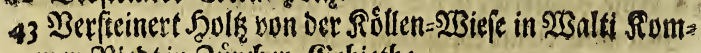
mer=Soledt in 3lirdser: Giebietbe.

44 (Eine nod) grine \$ommerange / fo berfeinert iff / neb fe. gmo andern attigen Eteinen / Davon einer/ als cine

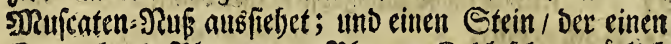
Etengel und Slume / von Slumen=Sobl febt natirlich vortuilbet.

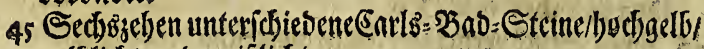
getblicht unto weiplicht.

46 Ein Eroftein von sild offisteim in ber Braffifoaffi fyamau.

77 Aletige geloe und rótblib) Steine / aus bem Egerijon Sauct=83rument.

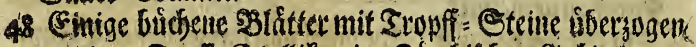

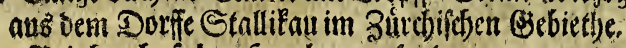

49. Seinfruch)/ fo bey Jeambatrg gefunden.

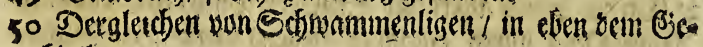
biettje.

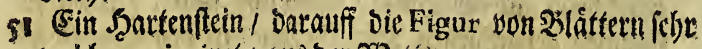
wobl exprimiret/ aus ber 2 setteraur.

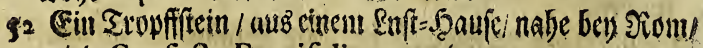
mito Confect Fontifoli gennmut.

33 Dergleidbeu Iropffftein muz Italien.

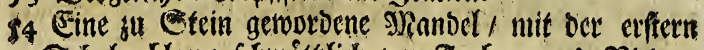

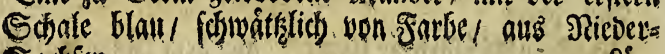
Sadjict.

Is Rü 


\section{(183)}

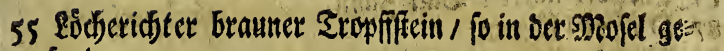
funben.

s6 Dergleiđen weip.

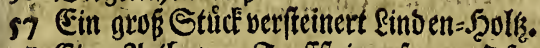

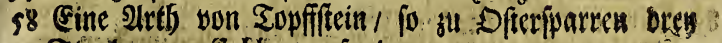
Sfimben von Eoblenz gefunben.

59. Tropfftein aus 23 aumann: Sçoble.

6o stiverer uit feiner Nutter.

61 Inderer Sroffftein aus beffen.

62 2noerer alts $353 i$ baben.

63 Dinderer aủ 5 Bnoen.

64 Inberer aus bem Earls:-3nioc.

6. Inverer aus dem Scubeffoden Sade.

66 4. Stridf ftetuerne Ero=ectivamme won Ninfel.

67 7. Sture dito fleiner.

$68 \mathrm{i}$. Curallen= Sindie

69 2. 低une Dattel:Sern.

70 Bitie Cartemome fouber mit den Stichl.

7r Ein gruiner fobmather Stcin/posine accurat vetfers

nerte SRiere vorffellet

72. Ein Rnodjen mit Tropffeen libersogen.

73. Sropffiten mit cinem faubern Slatt bon Sena.

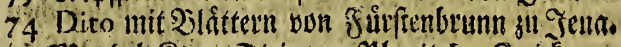

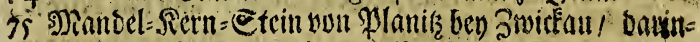

nen bie weiffen s) anedn in gelbet Shaut liegen.

76 - Maned = Serne fo nusgerdingen fins.

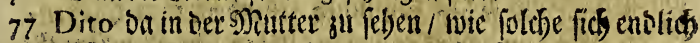

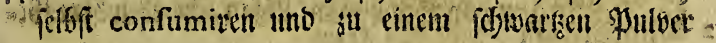
werien.

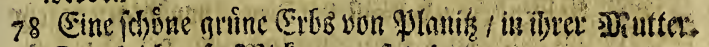

79 Detglcidien jo 2 Biden prefentiten.

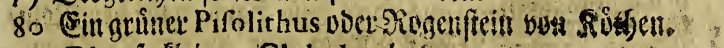

81 Dito fo fleinere Globulos bot.

82 3wen artige boble Tubuli.

83 Ein fdrwartiet Stein billoet eine Nietc.

84 I. Stuit petrificite Eorall. 


\section{橉 $(184)$, 新}

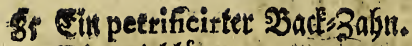

87 Dito viel lainger.

87 (Ein Vertebra von einem Sifo) von Giper|tadot.

88 Ein Stide Marmor mit einem unbetannten Sinodjets yon Duerfutth.

89. Dito mit einem anbern Sinodjen von Efperffádt.

90 Dito mit einem ífoinen Sinoden.

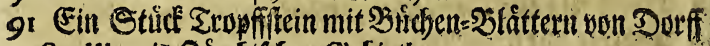
Scailicn in Zuircbifdien Giebietb.

9: Ein Sropfifizein io bey Dfterpar 3. Sfunder won Eo. blents gefumben wirb.

93. Ein fefter graner Stein/ ba bie eine fladse mit felse

icjoinen Sternen bepleibet ift won Camftadt.

94 Ein geaner Noúffein nit cimem artigen Sundjen von Duterfutity.

95 Eine Chalcedon= Siugel von $\$$ Planitg.

9s Curiöfe Ero = Steine bon SRambutre.

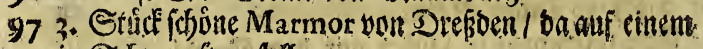

cin Stjwan pegn por.

\section{2inbang!}

DSeftehet in' Dertsecthnif einer Nenge allerhano

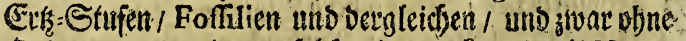
Srotumg / nut twie man foldbe eingepadft und mit Nummern Geseichnet gefunden.

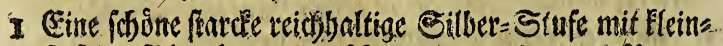

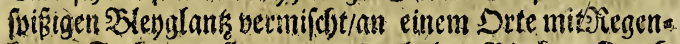

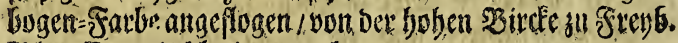

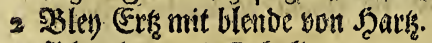

3 Slenglan mit Sobolo vecmengt won Shnaterg.

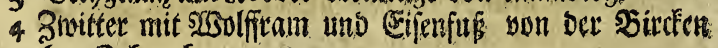
bey Sthneebers.

5. Rupffer: Ent in grauen Spath nit weiffen Rief von Saalfelbt.

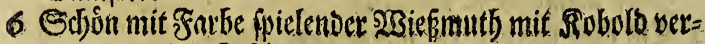
mengtuon ire Sdlem. 


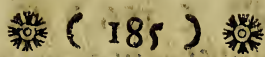

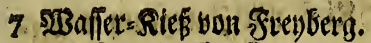

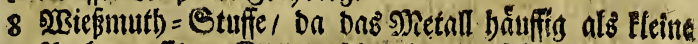
Merlen auff bem Stein aefóntulizen u fefien.

9 (Eine reiche $23 i e$ mutly mit foboner blauer Jarbe ange flogue Stuffe von Sdjneeberg.

io Eijenftein von ber Gottes Sjabe.

1I Siechbaltiger 2 Bießmuth von Jobann (Jeorgen Stabe.

12 Blaks=sopff oon Enbenfto of.

${ }_{3}$ Grobjpifiger Sblenglan's mit einer nngeflognen griute uno gelbe von Gopilar.

34 Bologelbes Sinpfier= Erts in weiffen Beftein yon Canlfelot.

Is Sinpffer-Nief yon foreyberg.

I6 Spurftein won Bettenborff

17. Rupferftein bon Sull.

I8 Siobulb von Frevberg.

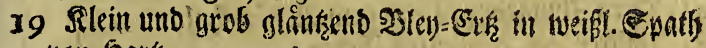
yon Sonts.

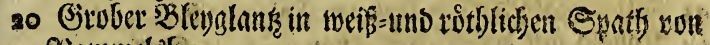
Stammelaberg.

2I 2 Baffer= fite

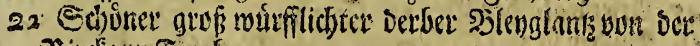
Bircte su frenberg.

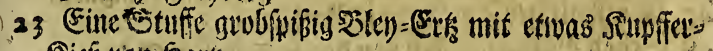
Rién von gath.

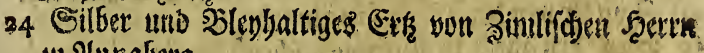
jull Alunaberg.

25 Antimonium.

26 Şraune Simpferfisladfe won Sostenoorff.

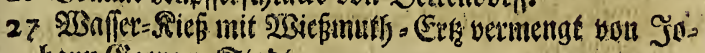
bavn Scotgen Stabt.

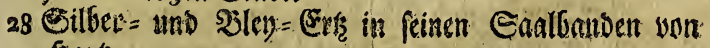
5ark.

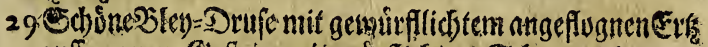
nuff genuten Bieftein mit wrinflidgten Echmarags. 3o. Sleyglan's von Jirenberg.

$$
\text { खs }
$$

31 Ediva 


\section{(186)}

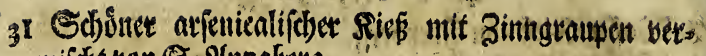
nuifdit bon 8 : Innaberg.

32 (Sin reicher Zwitter mit 3inngraupen son Innaberg.

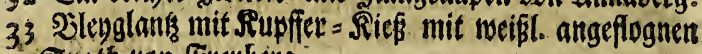
Epath yon Frenberg.

34 Siobolo von sBiefentfal reicisjoaltig.

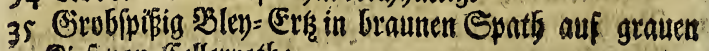
Sicé yen Sellerrothe.

36 Farbern Siobulb von Der Saglette.

37 sibbuld von grenberig.

38 Gifenfdoufiger 3witter.

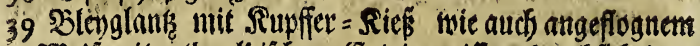

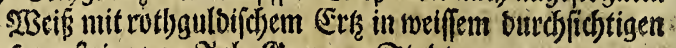
Şornffein/ youn Yol. Beotgen Stabt.

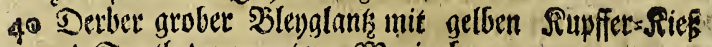
uno Spath sermengt yur 9 Rarienbers.

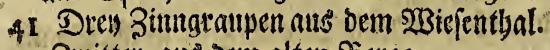

42 3witter aus Dem altert Sierge.

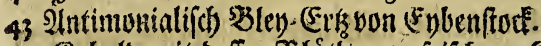

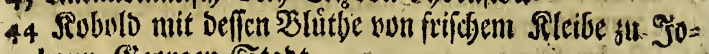
bam Gieurgen=Stabt.

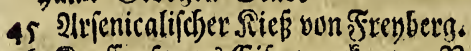

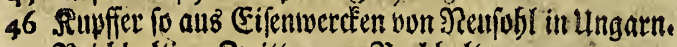

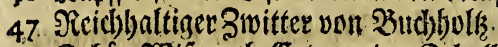

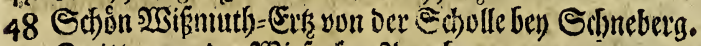

993 woitter ven Der 23 Sicic bey 2tnnebera.

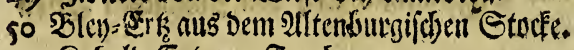

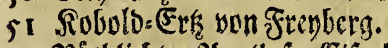

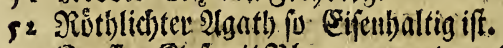

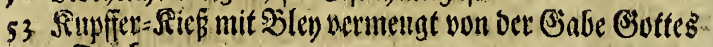
zu Sreenberis.

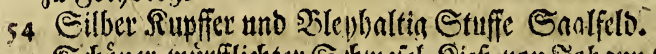

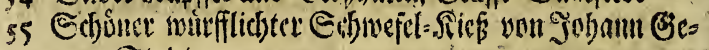
uraen=Striot

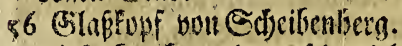

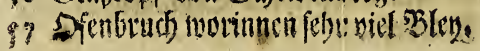




\section{煞 $(188)$ )}

86 s3olfitintio

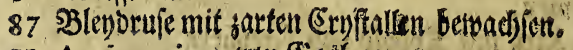

88 Ancimonium von Goslar.

89 Sieidbaltiger o uno D Eefiefer von Jimennu.

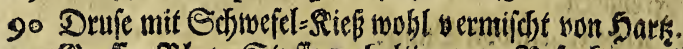

91 Broffe Bler = Stufie D baltig von Siofentrans in

Frenterig.

92 Eelje flarer Sillatraltiger \$leyglang.

93. Marcafic in (Eifenfitem bey) Schwartzenberg.

94 Blenbrufe mit Eryffallen yon Sarts.

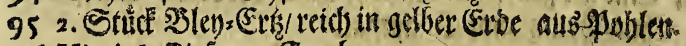

96 Virriol-siés yon Frewherg.

$$
\text { Der 4. Liaften. }
$$

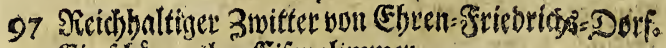

97 Ein foun rotber Eifenglinmer.

98 Rupfert Nię von Suble.

99 शE lenve uon Frenbers.

100 31witter won Etfeibenberg.

101 Śblate von Dem Antimonio.

102 Antiatonialifose Sdjmefet-Sief.

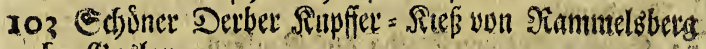

bey Singlar.

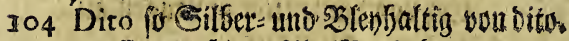

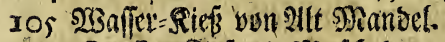

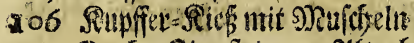

Y07 Serber Simftein you Alltenberg.

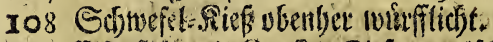

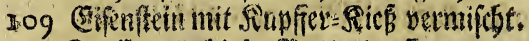

no Rupfer: Soldefer-3ottenoorf.

II I Secicher Zwitter von bet Shlette.

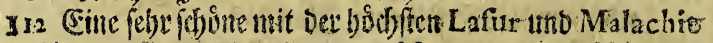

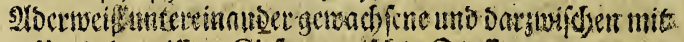

gelb= tutb weifen Riefs vermifolse Stuffe:

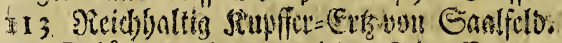

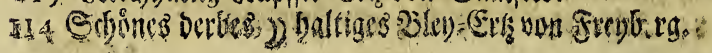




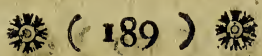

I5 Marcalit uon Sinnftoorf.

116 Antige Sley= Druie.

117 Ġlatis= Ropf von Jundobuiba.

I 8 Stablftein wou fichtellberg.

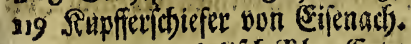

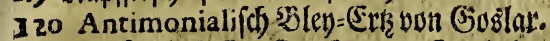

izr Sdion Derb Siluffer=Erts bon Saalrelo.

322 Fobolo = \$llithe von Sdinebers.

123 Sieiduer Snitter bon Sd)lactenualoe.

324 Eine rare Stuffe won angeflogem Silber wie die zars

fien 5 ante.

125 Ein gelber Eifenftein won ber Seertuleib.

126 Ein reidfer Vitriol-Sies.

\$27 Esiner wurfflidtet Sdjefel=Sieß,

3er zaften: Zllerley 1 erge.

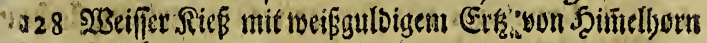

zu frenberg.

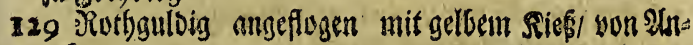
nebers.

30 Weifgulloig non Edjnebra.

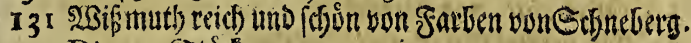

132. Dito 2. Stude.

I33 Dito 3. Stride.

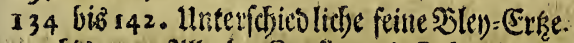

143 fit 152. 2lllerlen Supfier und Sofwefel= Riebe.

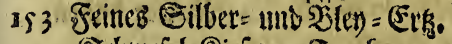

I54 Edwefel-sies von fret)

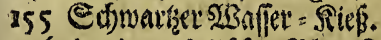

156 Antimonialif(i)er Slet)= Siep.

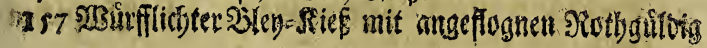

Der Kaften : Mancberley Steine.

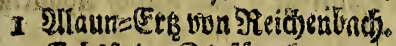

- Qubofteine Staffurtí.

3 Fin Stuce Achat ofen mit artigen \$udelln.

A Drey uerfeinerte SRujojeln Roftrate genamut.

ร 3 เมต 


\section{繁 $(190)$ )}

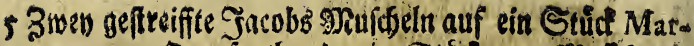
mor von Duerfurtb) it. ein Sticf von SRaffel mit Iftriatis.

6 Ein rar Stüf ba in cinen farten meiffen Marmor Dato telfeen eingewa ój)en find.

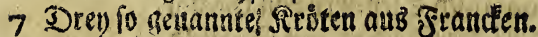

8 Etliche (Er)fteine aus ber Reimgrube su शRaumburg.

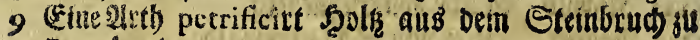
Sueviurtio.

to Dito fo miloer wie Imiantf.

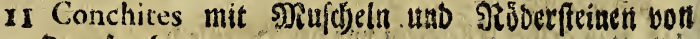
Quserfutt).

12 Drey Duplet ober fo genanate fiteufifteine vott dito,

13 Chynelifter Sped: Strin.

34 Cir Stúte rober Alabalter.

15 Esin Conchites mit felbr artigen georefyeten flacbert Situritien.

16 Cin idoin bunter Agath von (Sland)e.

17 Syacinth Fluß von 21 neberg.

18 Ein curififer Tophus.

19. ORteocolla von SRaffel.

20 (Ein velfer Spath von stamaterg.

21 Sand $=$ Ers mit einer fojsunen Lafur $=21 b e t$.

22 Spaath ans ber Sofwein.

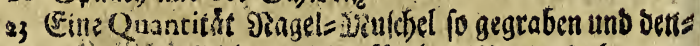

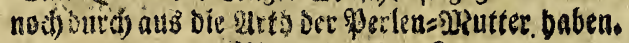

24 Chalcedoni von Planif̧ bey zmitau.

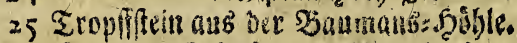

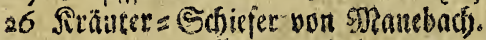

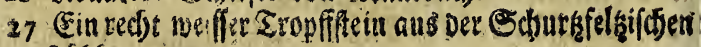
Şivble.

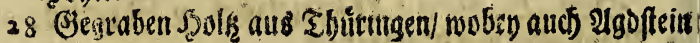
gefuntern toiro.

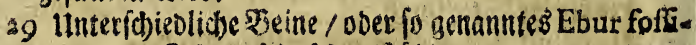

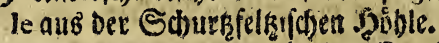
36 Dito efrandere Dergleidern SReocera. 


\section{粼 (}

3I Dito nod) 3. Etride.

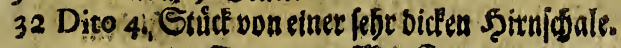

Der 2. Zaaften.

:33 Gin Strid Vitriol bon Stjouna.

34 Concha roftrata aus der Sojpreifig.

35 Sin Seurerftein fo mit vieten fartben als ein Agath ipielet.

36 Pleine \$ierg:-Eroffallen.

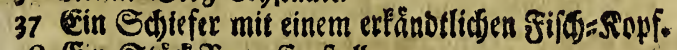

38 Ein Ettut' \$erg: Eryffall.

39 Gin rob Stuich Marmorbon (sibdienftein.

40 Etlide petrificirte \$Rufdeln von Duerfurth.

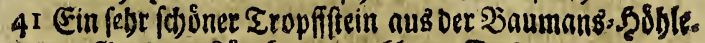
42 Gipg von Ruineburg/mit blauer Sarbe.

43 Ein DRuffoelftein von Efperftâdot.

44 Dito Pleinere.

45 Ein róthlidter Spath.

46. Lac Lune von Şark.

47. Jübelizet Serpentin-Strit.

\section{Eedffe Oronung}

Scalt in ficic) von bem

Vegetabilif(ben YReid)e, austånsifde uno vare Dourneln/ Sdjalen und Kinden/

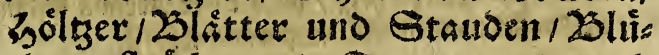
then / fruidte und Gaamen/Gummi, bårze und Gâffre/ und andere $3 u$ fols d)em Zreid)e 3 urechende Dinge.

Unslåndifd)e rare รBurseln.

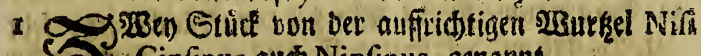
2. Ginfinus auø Ninfinus. genannt.

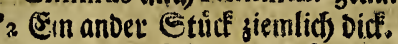

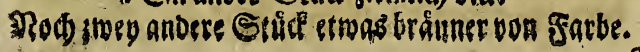




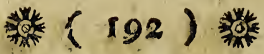

3 Now) cin anderes faff braunes.

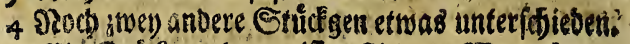

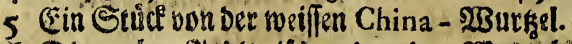

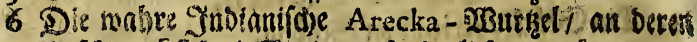

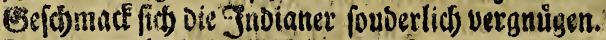

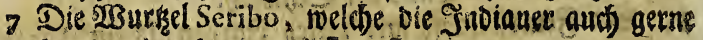
beil Der Arecka ö genieffien pilegen.

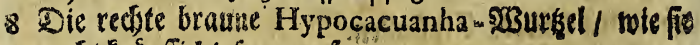
recht Enippfictet fegn müs.

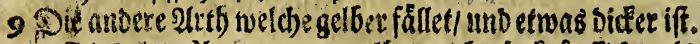

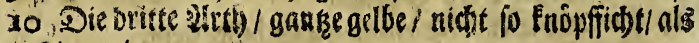
Die votige.

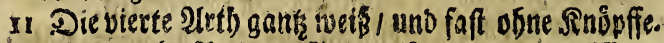

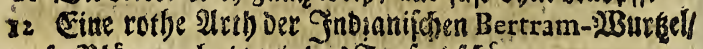

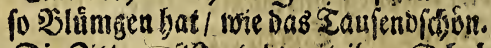

13 Die Bittwet=213uthel/ mit ibrer Sobale.

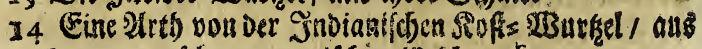

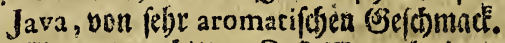

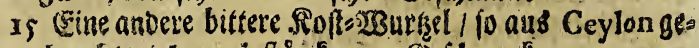

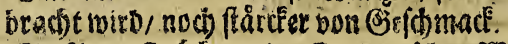

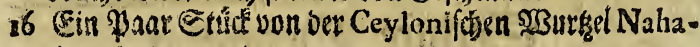
dopula geneutuct.

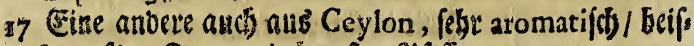

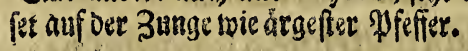

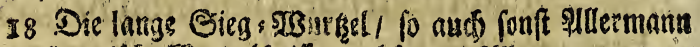

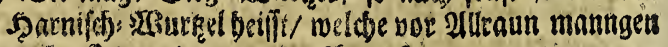
yure? atffet meroen uno migbraudjet.

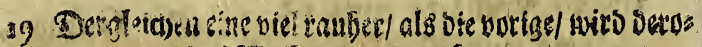
wegen wor Das vascibgen ausigegeben.

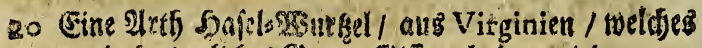
vor er fouberlideses Begen=Esifit gelbalsen wirt.

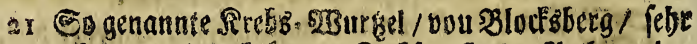

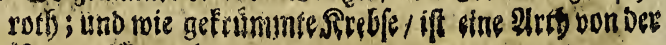
श)atter-20 


\section{新 ( 194 ) 橉}

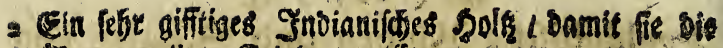
5zrunuen ibrer Seinbe vergifiten.

- Das wabre Steins Solk/ Doer Lignum Nephriticam,

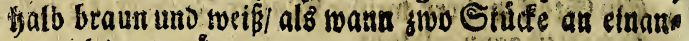
Der geleimet woiren.

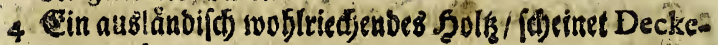
roli jut fenn.

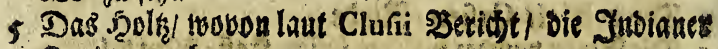

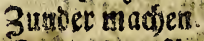

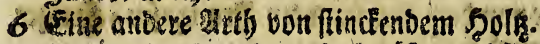

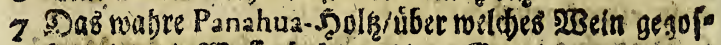

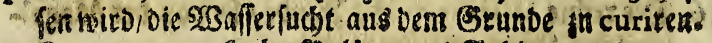

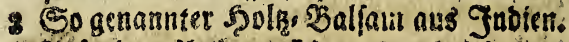

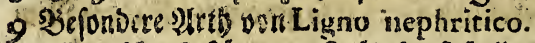

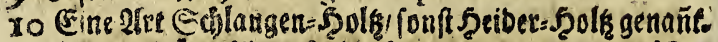

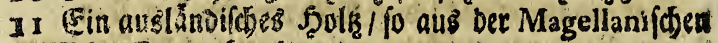
SoReer:-Enge sebradit wird.

12 S?och etne Altib von Ligno Panahua..

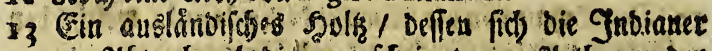

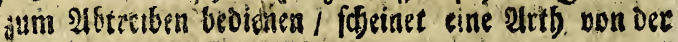
Subina.

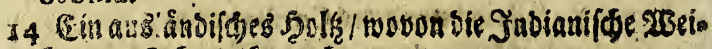
ber ijere Edominte madjet.

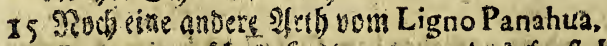

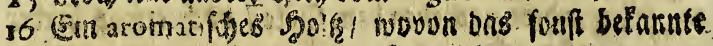

Oleum atomaticum gemawet mitro.

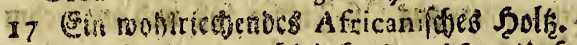

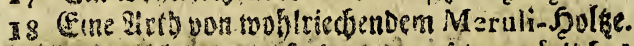

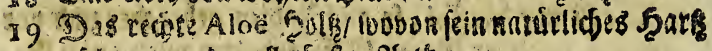

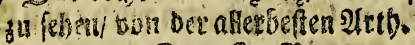

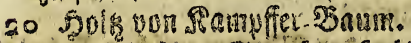

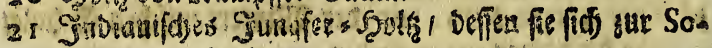
phiftication bevicener.

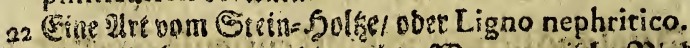

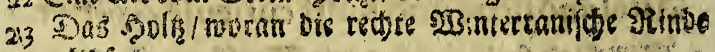
to:

34) Ein wooflr: 


\section{( 195$)$}

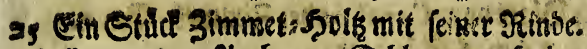

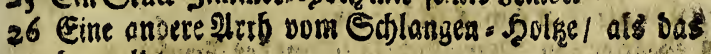
obgemelote.

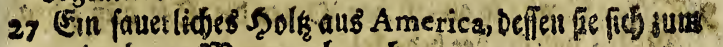
netborbenen MRagen gebrauctien.

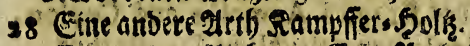

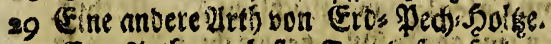

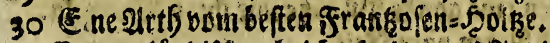

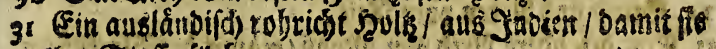

* thre Et t ffe färben.

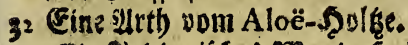

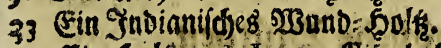

34 Ein Şolf nue Javal Eifenbolts ober Metrofidros geneunt.

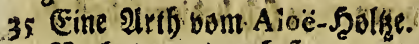

35 Rod cine anbere beffere.

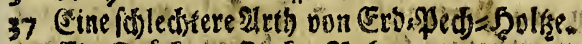

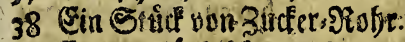

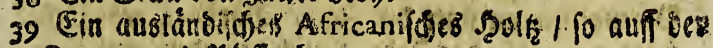

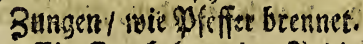

40 Ein Stachel rou ber Inbianiforen Acaris, welche bia

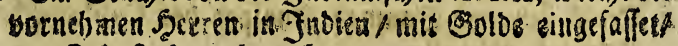
zum 3asnffecter gebraudfers.

4I Lignum Safifras.

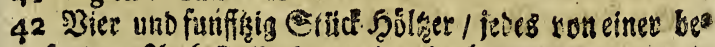
foniert Srth Cylinder artig geberbs.

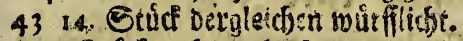

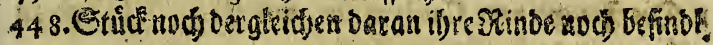

Zublindifhe Státter tns Standoen.

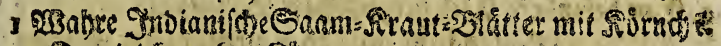

2 Dergieithen abne Fistrer.

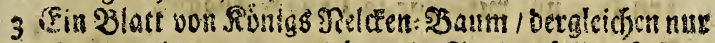
einer/ mie mant vorgiebes/ in Gimbien forl gefurbers imerien.

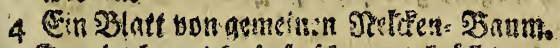

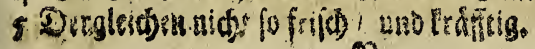




\section{(196)}

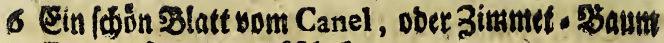

7 Dergleidinin etwas follediter.

8 B:atter von SNeet, (Eid)eln / famt ber Frnofit.

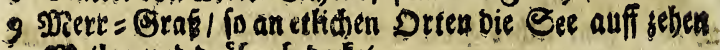
smetlen und srifíber bebectet.

10 Einige Blátttet Des Srautes Acmelle aus Ceylon,

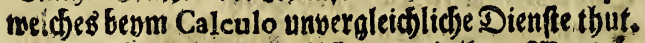

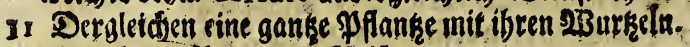

1s Exroh und Pleten uom Treif.

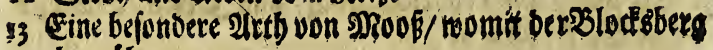
oben suberzogen.

\section{Zusländifche 2slitben.}

i Sliteffe von SRuf ater.

- Die belannte Moxa / Deren man fíd bebienet dab Pos dagra zu curiten.

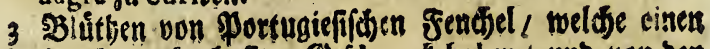

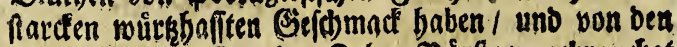

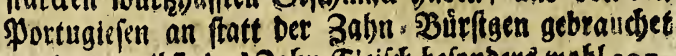

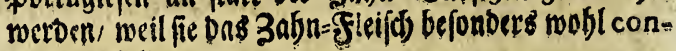
ferviren follen.

4 Die Bilíthe und Saamen= Snofpen Des. Ceyloni(đen Rrantes Acmallix.

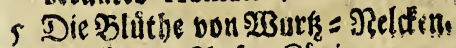

6 Ein Heiner Difen Soutig.

\section{Xud}

I Die Fructis von Cardemum/ wie folthe in befonbern Capfuln an Stielen wad) fen.

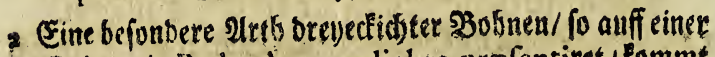
Seiten cin Pudendum muliebre prefentitet / fommt von Java uno mito Buoca Pange genannt.

3. Eine ummenseine groffe Miscafen Nuß / bergleidjen an Gróffe nod) nirgen's genefen / 3. Foll lang uno $\frac{1}{2}$ 3oll breiţ. 


\section{粼 (197) 粼}

Die Sradet vom \$oll6aum / mov eitue jiemlidje Quan. titåt SBaustubolle / mie fie reiffet zu feben iff.

5 Sine befondere 2Irtb won Chinefifon Pflaumen / fo

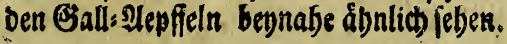

6 3wey anberer 2trth aud) aus China, feben unfern une

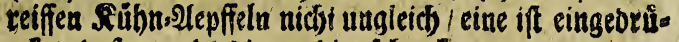
ofet / Daß man Das inmenbige felfen fon.

- Die Inbianifoe Frudit Lanith, if ungenein bitterl und wirb vonben Inbianern a als ein befonder Medi. cament swiber Ëiffit = Fieber und Dergleiden geo Braudet.

- Die Fofnen des Şeiligen Ignatii, babon einige neue Auctores unter[dyieblidjes gemelbet.

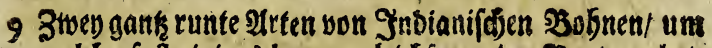
meldbe faft einiges berum gleidjam eine sorte geljet; sirt fonften Cahara genanut.

so Cine groffe $\mathfrak{B}$ ohne bes f̧eiligen Thomæ, beren bie Inbtaner fids zum Purgiren bedienen / bat auff benden Seiten eines Pưféel.

II Eine andere weldse glatt; tui braun rotflid vom Sarbe.

12 Eine andere fleinere / fo an ben beeiteften Drt ange: feffen / bat faft die Figur eines Şer/żens.

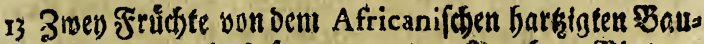
me $/$ monyon Das fo genanten Eradjen = \$llut gee macjet wiro.

14 शRod) eine Tlomas = \$obne faft Circular runbtl Darbey noc) eine fleine mit 2. Purfeln.

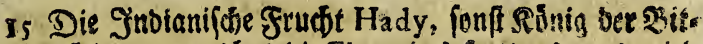

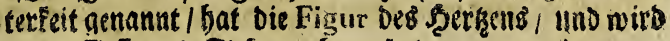
wiber Sifft und Steber gebraudet.

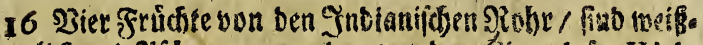
(id) und 2l(d)greu / werben uon den Cingalef̧on Walgamba genannt.

$$
\text { श } 3 \quad \therefore \quad 17
$$




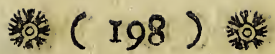

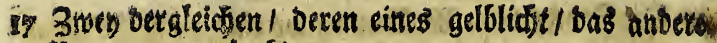
Pommerantgenfarbia.

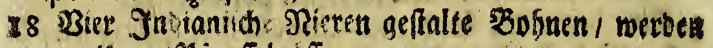
von ignen Pia ffelmilf: genenner.

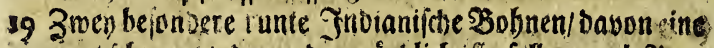
gankidamars/ de anoere rótblid ift ; follen auch Purs giers sisobnen fepn.

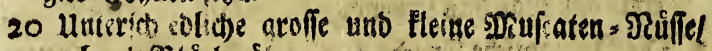
nid mit Sbluthe í es sen.

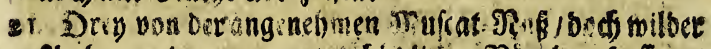

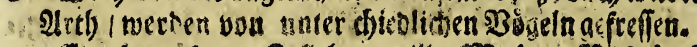

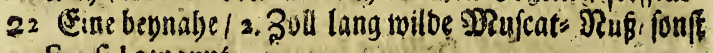
Frufigenannt.

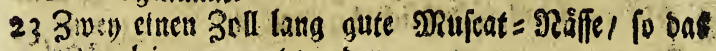
Gatullin genannt werden.

24 3 ven anderc monftröfe MulcatsRéffe.

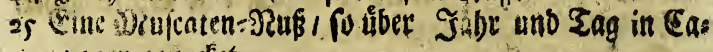
paraen ge ifefert.

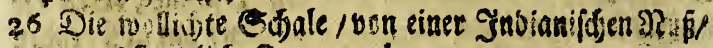
barans fie ellich? Zenge madsen.

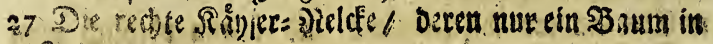
Intiea.

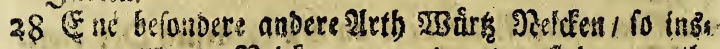

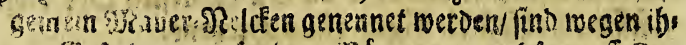

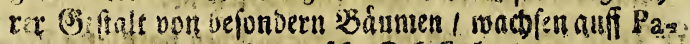

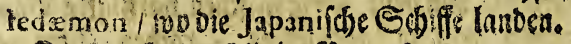

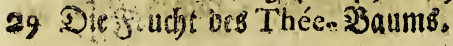

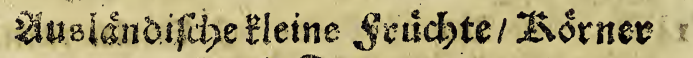
mo Sarmen.

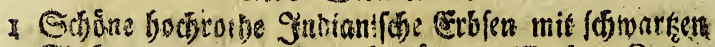

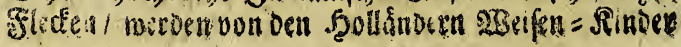
genemet.

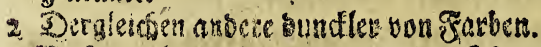

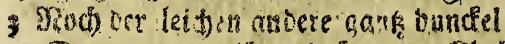

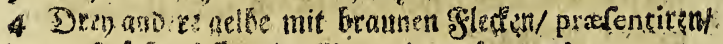

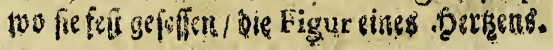




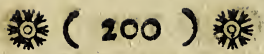

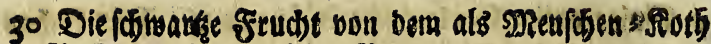
filndenden Sinbianifisen Baume.

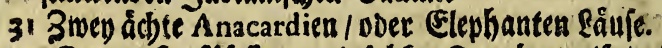
32 Der radfite \$foffer/ wie foldfer Eraubentweife in Swo Dien imadiet.

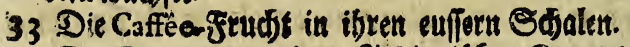

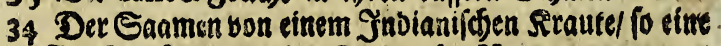
Frudft trdiget / wie ber Cingalefen U racu.

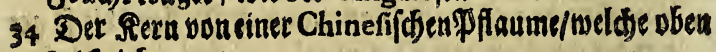
be (d) riebera.

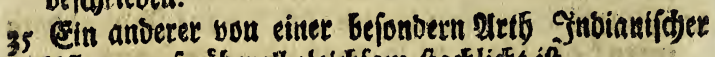

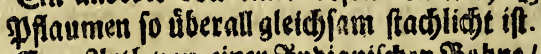

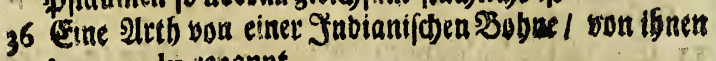
Apanapalu deannt.

37. Eime be fondere 2Irth von Mirabolanen.

${ }^{3} 8$ ร20

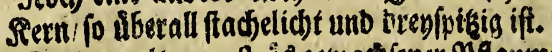

39. Ein Doppelf monftris gefna d fener PP Paumen-Sectn.

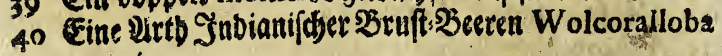
genannt.

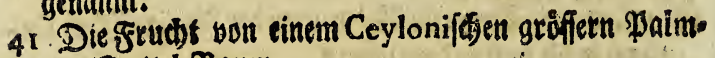
Doer Dattel, Baum.

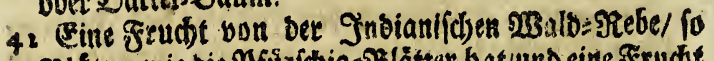

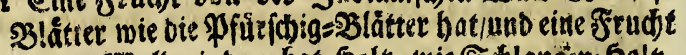

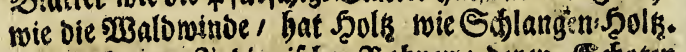

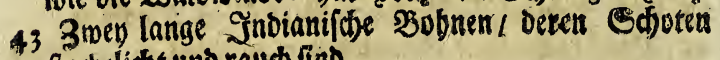
fladtelidst und rauds find.

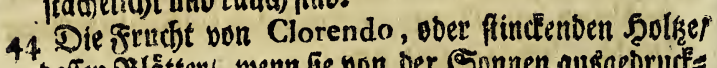

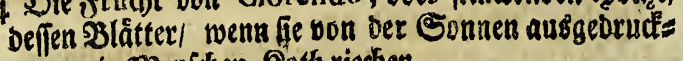

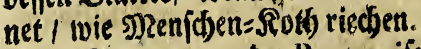

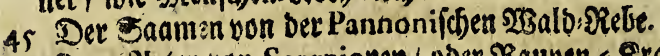

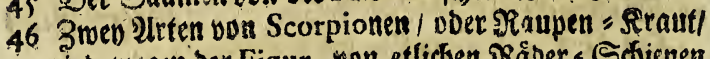

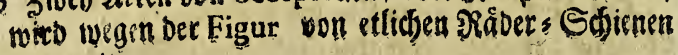
gettannt.

47 Eine andere Uleth bon bergleiøen.

48 Now 3 iwo andere 2 Atten.

49 Die Srrudet son Thamarindelt. 


\section{紫 (201) 彞}

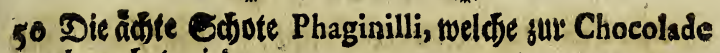
gebraudetet mirb.

5I Die gruudit von Dem Ceylonifosen moblriectjenden

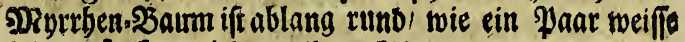

Dolrgebảnce / wirs von ibnen Hincupua genannt.

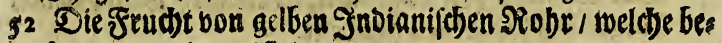
fonders artig ausfichet.

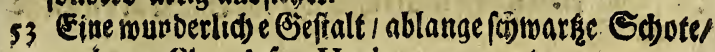
yon betten Cingaleén Hutharu genannt.

54 Eime andere rotfe/ won ben Cingalejen Amparu genannt.

5) Eine SRufcaten s Sur / mit iljeten 3. Sdjalen.

s6 Bmen befondere Scjoten/ von benen Cingalefen Lac。 berge genanmt.

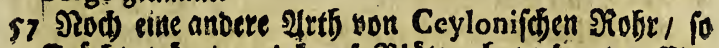
Gruidite tráget / unb traufe Blấtter bat / ben ben Eins ländern Ghuprat gettannt.

58 Der Sanme yon einer bejonbern 2trth Indianifbee

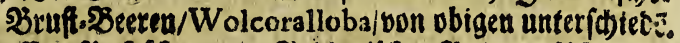

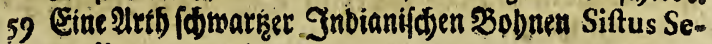
nordius genannt.

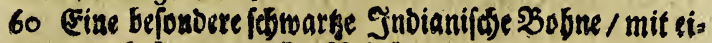
nem abobenen weififen SRabel.

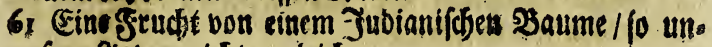
Pern Einden nicht ungleidi.

62 Der Saamen upn der S̈ntiani(đ)en Frudfit Uracu. 63 Der Ceylonif(te Saamen Polleghipaganelli, bit) Ben Cingalefen genannt / mit feetuer bejonbern Ed mie eine 2 blǘtbe geffaltet.

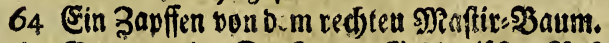

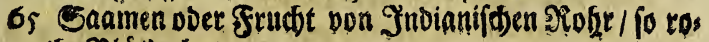
the Sblithe bat.

63 Der Saamen von ben Sintianifoten Citrullen.

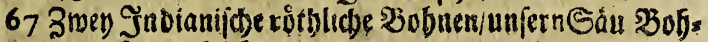
neu nidst ungleiof.

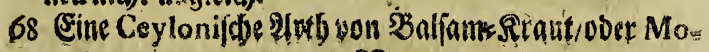

$$
\text { R } 5 \text { mox. }
$$




\section{然 (202) 䊁}

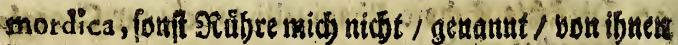

Kurhala genernet.

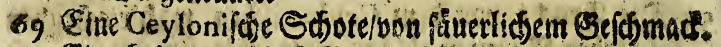

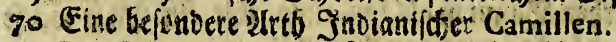

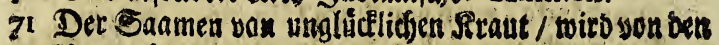
Cingale eren Pinathola genanut.

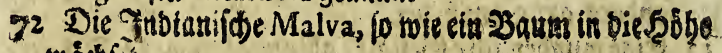
wáchfiti.

73 Monftrȫe Şrajeltrúfie.

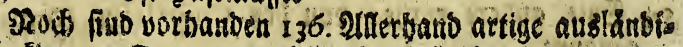
fore Ele nere Saamea / meldje alle zu beldo:eliven ju meits láufitig futlen iolte.

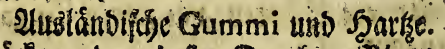

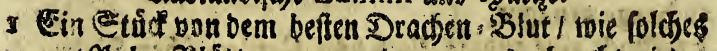

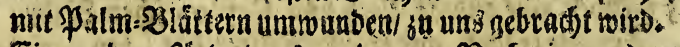

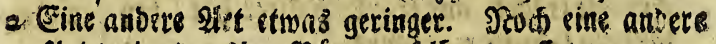

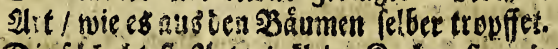

3 Die fdirectetefere Strt toic Eleize Suden formitet.

4 Das Gummi \&ali $/$ mise es in Taffelin aus Inbien ges bracte twitb.

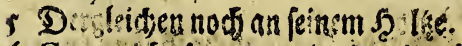

6 Girmmi La danum, wie es ordinair ift.

7 Gummi Ladanum, wie es in ben Ziegen × Bátrtben the fammen getragen wiro.

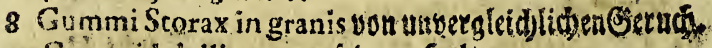

9 Cúmini bdellium, an feirem follge.

10 Gine iefoniece alt von bee Affi foetida.

II Daf aid)te Ciummi guttas.

12 De reshic Ballam won Meccan

13 Der wabre \$illian von Tolu.

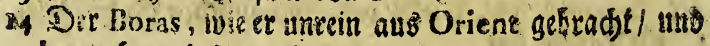
berna (⿻) raviniret mirs.

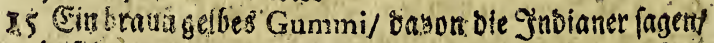

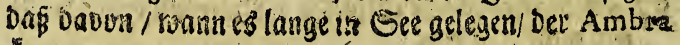
fonme.

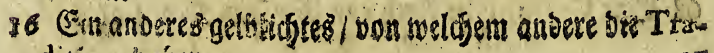
dition bajsta. 


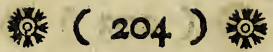

moran feine Sinde nod über und úber su felten. 4. unt cin ball fắ lang recdis gerabe.

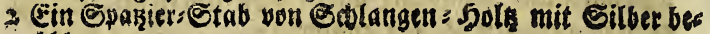
feblagent.

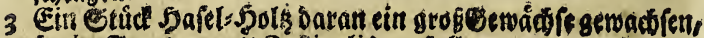
fo eint ocrofum mit Eefictulis vorffellt.

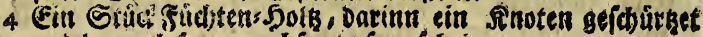
uno beruach po verwadbien fu fegn icheinet.

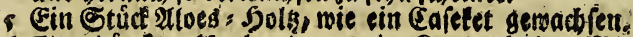

6 Eine bürdene squtbe, Daban eill Nnoten in ber MRitt ges wachien.

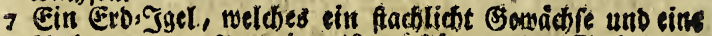

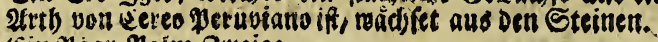

8 Sin \$aar Maim:Bweige.

9 Etliche Reiffer voun Neldens? Baum.

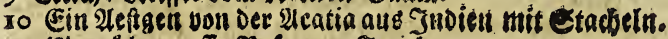

ni Eine febre qroffe Siofe von Jerido.

I2 Gine nindere etroas tlener.

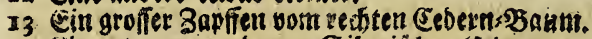

14 ein anderer von benen Siberichen Etoern.

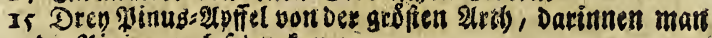
Die Minien noch fel)en tan.

16 2. 220ams: 2tpffel.

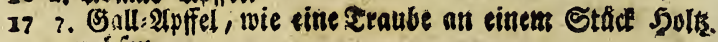
getwachien.

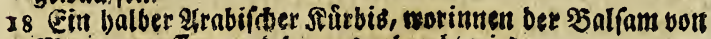
Decca gegafien, unto beraus gebrecht wiro.

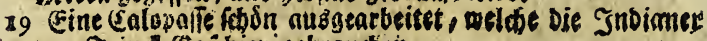
3urr Irind 'seicoier gebraudien.

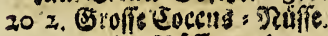

21 2. Yalm: Siaffe noch gans.

22 efine auffgefontittene dito.

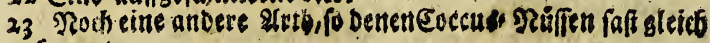
tomunt.

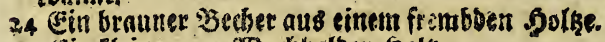

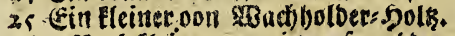

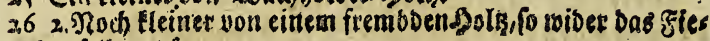
ber foli gu: ienn.

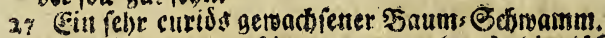

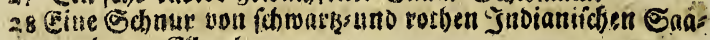
men bey 4 . Eillen Inna.

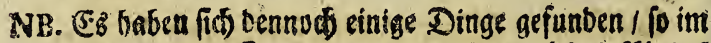
Catslogo vergeffen/ uno megen Mangel Des \$lakge nicjt eingeruidet merben ESonen.

(E) D $D$. 


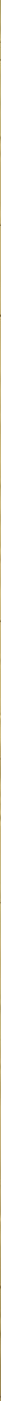


B. 1 ,

$i=\quad i$

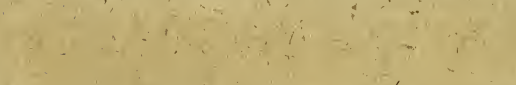

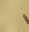

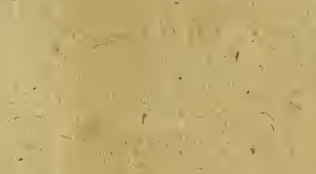

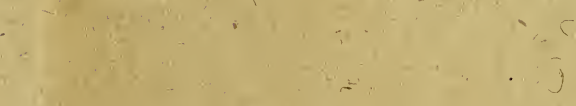

$5+2$

$+\infty$

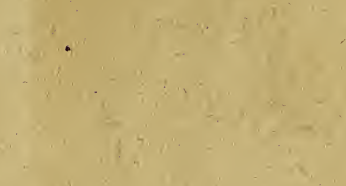

$-1$

$\rightarrow$

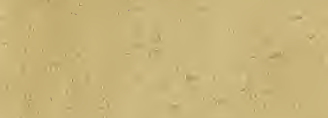

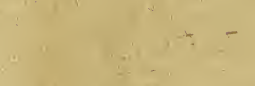

$1+1,+1$

$\sin x$
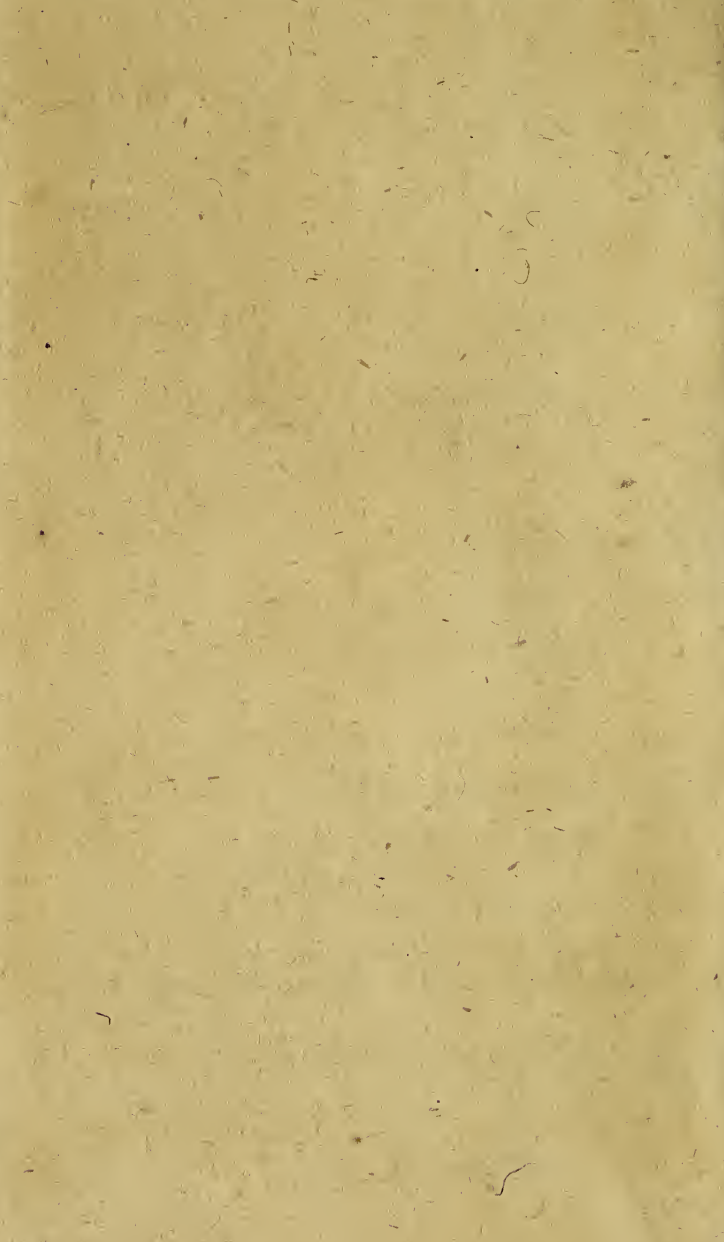


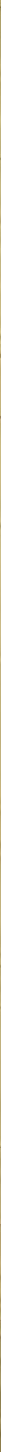




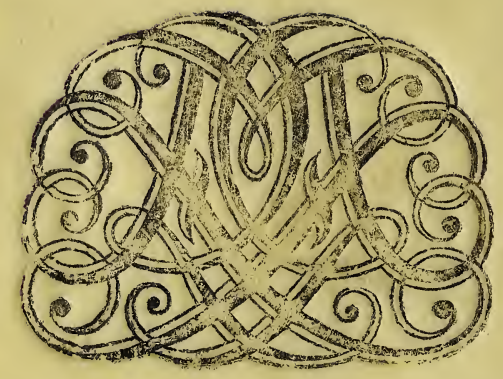

